Florida International University FIU Digital Commons

3-29-2011

\title{
Clinical Translation of a Novel Hand-held Optical Imager for Breast Cancer Diagnosis
}

Sarah J. Erickson

Florida International University, sarah.erickson@fiu.edu

DOI: $10.25148 /$ etd.FI11050315

Follow this and additional works at: https://digitalcommons.fiu.edu/etd

\section{Recommended Citation}

Erickson, Sarah J., "Clinical Translation of a Novel Hand-held Optical Imager for Breast Cancer Diagnosis" (2011). FIU Electronic Theses and Dissertations. 407.

https://digitalcommons.fiu.edu/etd/407 


\section{FLORIDA INTERNATIONAL UNIVERSITY \\ Miami, Florida}

CLINICAL TRANSLATION OF A HAND-HELD OPTICAL IMAGER FOR BREAST

CANCER DIAGNOSTICS: IN VITRO AND IN VIVO TOMOGRAPHY STUDIES

A dissertation submitted in partial fulfillment of the

requirements for the degree of

DOCTOR OF PHILOSOPHY

in

BIOMEDICAL ENGINEERING

by

Sarah J. Erickson 


\section{To: Dean Amir Mirmiran}

College of Engineering and Computing

This dissertation, written by Sarah J. Erickson, and entitled Clinical Translation of a Handheld Optical Imager for Breast Cancer Diagnostics: In Vitro and In Vivo Tomography Studies, having been approved in respect to style and intellectual content, is referred to you for judgment.

We have read this dissertation and recommend that it be approved.

Wei-Chiang Lin

$\begin{array}{r}\hline \text { Chenzhong Li } \\ \hline \text { Joong-ho Moon } \\ \hline \text { Anuradha Godavarty, Major Professor }\end{array}$

Date of Defense: March 29, 2011

The dissertation of Sarah J. Erickson is approved.

\begin{tabular}{r}
$\begin{array}{r}\text { Dean Amir Mirmiran } \\
\text { College of Engineering and Computing }\end{array}$ \\
\hline Interim Dean Kevin O'Shea \\
University Graduate School
\end{tabular}

Florida International University, 2011 


\section{DEDICATION}

I dedicate this dissertation to my father, Matthew Erickson, who has given endlessly of himself for his family and has always supported me in so many ways. 


\section{ACKNOWLEDGMENTS}

I am grateful to be surrounded by so many amazing people who have helped and supported me throughout this process. Dr. Jiajia Ge initially trained me to operate the optical imaging system and provided help and encouragement in every aspect of my research along the way. Jean Gonzalez, Sergio Martinez, Annie Nunez, Manuela Roman, Lizeth Caldera, and Andrea Sanchez assisted in so many ways during experimental studies and processing data. As a fellow PhD student, Ujwal Chaudhary provided support and a different perspective on my research. All the past and current members of the Optical Imaging Laboratory including Dr. Banghe Zhu, Steven Regalado, Adrian Romero, Joe DeCerce, and Michael Hall have helped to make the research possible. My dissertation committee members Dr. Wei-Chiang Lin, Dr. Chenzhong Li, and Dr. Joong-ho Moon have provided insight and guidance toward my dissertation. I would like to acknowledge the university graduate school and the Department of Defense for funding me and the National Institutes of Health, the Coulter Foundation, and the Florida Department of Health for funding the research.

I am eternally grateful to Dr. Anuradha Godavarty who has been an outstanding mentor for me. Her stringent yet patient guidance has molded me into a high quality researcher. She gave so much of her time and effort to advise me in the research process, teach me scientific writing, grant writing, and IRB procedures, and cultivate me towards a future career in academia. My current and future success is greatly attributed to her.

I would like to acknowledge my family including my father, Matthew Erickson, my brother, Justin Erickson, my closest friend, Christine Esquinaldo, and my fiancé, Ruchir Bhatt for providing tremendous support and encouragement. Finally I would like to acknowledge my mother, Linda Erickson, whose memory continues to inspire me every day. 


\title{
ABSTRACT OF THE DISSERTATION \\ CLINICAL TRANSLATION OF A HAND-HELD OPTICAL IMAGER \\ FOR BREAST CANCER DIAGNOSTICS: IN VITRO AND IN VIVO \\ TOMOGRAPHY STUDIES
}

\author{
by \\ Sarah J. Erickson \\ Florida International University, 2011 \\ Miami, Florida \\ Professor Anuradha Godavarty, Major Professor
}

Optical imaging is an emerging technology towards non-invasive breast cancer diagnostics. In recent years, portable and patient comfortable hand-held optical imagers are developed towards two-dimensional (2D) tumor detections. However, these imagers are not capable of three-dimensional (3D) tomography because they cannot register the positional information of the hand-held probe onto the imaged tissue. A hand-held optical imager has been developed in our Optical Imaging Laboratory with 3D tomography capabilities, as demonstrated from tissue phantom studies. The overall goal of my dissertation is towards the translation of our imager to the clinical setting for 3D tomographic imaging in human breast tissues. A systematic experimental approach was designed and executed as follows: (i) fast 2D imaging, (ii) coregistered imaging, and (iii) 3D tomographic imaging studies. (i) Fast 2D imaging was initially demonstrated in tissue phantoms (1\% Liposyn solution) and in vitro (minced chicken breast and 1\% Liposyn). A $0.45 \mathrm{~cm}^{3}$ fluorescent target at 1:0 contrast ratio was detectable up to $2.5 \mathrm{~cm}$ deep. Fast $2 \mathrm{D}$ imaging experiments performed in vivo with healthy female subjects also detected a $0.45 \mathrm{~cm}^{3}$ fluorescent target superficially placed $\sim 2.5$ 
$\mathrm{cm}$ under the breast tissue. (ii) Coregistered imaging was automated and validated in phantoms with $\sim 0.19 \mathrm{~cm}$ error in the probe's positional information. Coregistration also improved the target depth detection to $3.5 \mathrm{~cm}$, from multi-location imaging approach. Coregistered imaging was further validated in-vivo, although the error in probe's positional information increased to $\sim 0.9 \mathrm{~cm}$ (subject to soft tissue deformation and movement). (iii) Three-dimensional tomography studies were successfully demonstrated in vitro using 0.45 $\mathrm{cm}^{3}$ fluorescence targets. The feasibility of 3D tomography was demonstrated for the first time in breast tissues using the hand-held optical imager, wherein a $0.45 \mathrm{~cm}^{3}$ fluorescent target (superficially placed) was recovered along with artifacts. Diffuse optical imaging studies were performed in two breast cancer patients with invasive ductal carcinoma. The images showed greater absorption at the tumor cites (as observed from x-ray mammography, ultrasound, and/or MRI). In summary, my dissertation demonstrated the potential of a hand-held optical imager towards 2D breast tumor detection and 3D breast tomography, holding a promise for extensive clinical translational efforts. 


\section{TABLE OF CONTENTS}

CHAPTER

PAGE

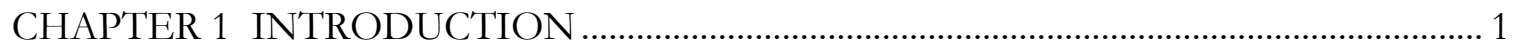

CHAPTER 2 BACKGROUND: NIR OPTICAL IMAGING ................................................ 8

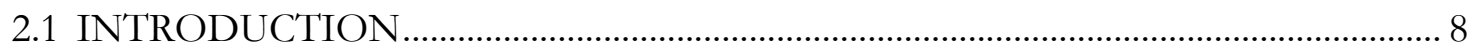

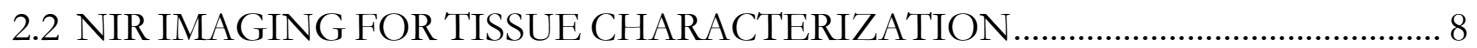

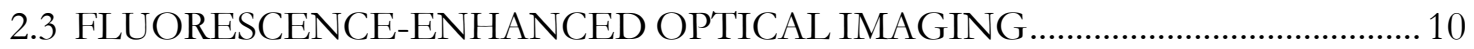

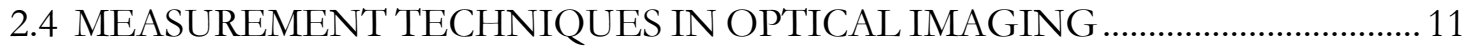

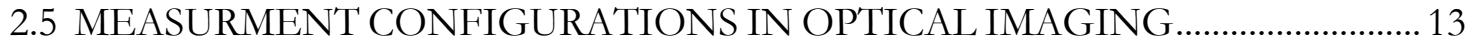

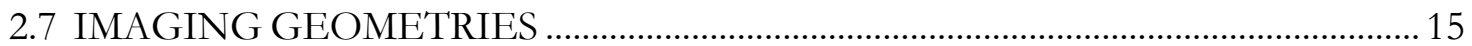

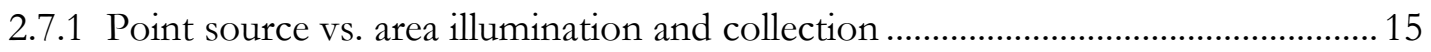

2.7.2 Simultaneous illumination using point sources to approximate area illumination

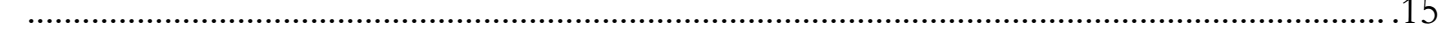

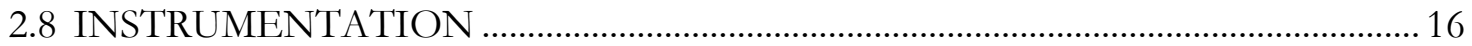

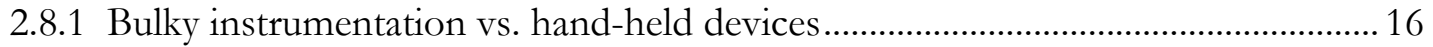

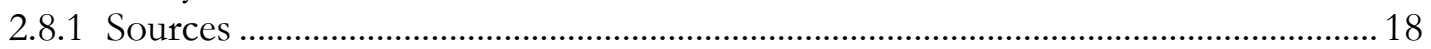

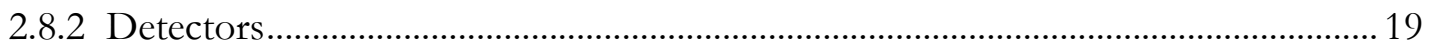

CHAPTER 3 PAST WORK: HAND-HELD OPTICAL IMAGING DEVICES ............. 21

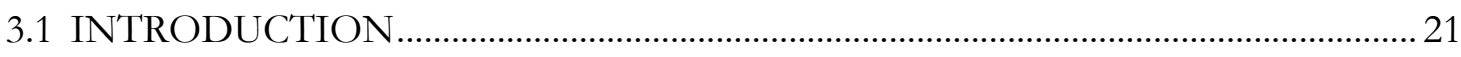

3.2 MEASUREMENT TECHNIQUES IN DIFFERENT HAND-HELD DEVICES

3.2 MEASUREMENT TECHNIQUES IN DIFFERENT HAND-HELD DEVICES

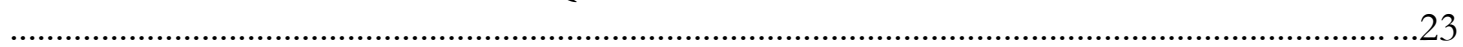

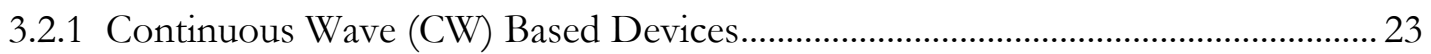

3.2.2 Frequency-Domain Photon Migration (FDPM) Based Devices .............................. 24

3.2.3 Time-Domain Photon Migration (TDPM) Based Devices ....................................... 27

3.3 IMAGING METHODS OF DIFFERENT HAND-HELD DEVICES ....................28

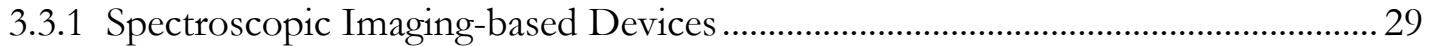

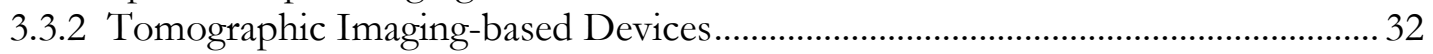

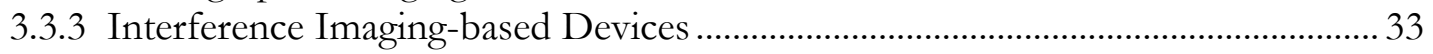

3.4 CLINICAL APPLICATIONS OF DIFFERENT HAND-HELD DEVICES ............ 34

3.4.1 Measurement of Normal and Abnormal Tissue Physiological Properties ............ 34

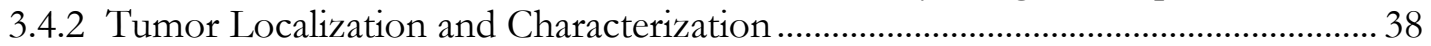

3.4.3 Monitoring Tumor Changes during Neoadjuvant Chemotherapy …....................... 40

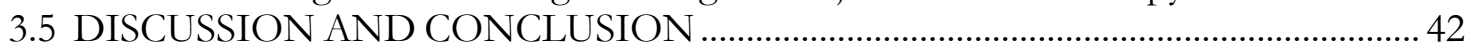

CHAPTER 4 PAST WORK: FLUORESCENCE TOMOGRAPHY EXPERIMENTAL

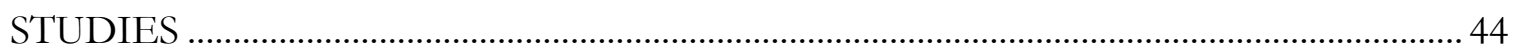

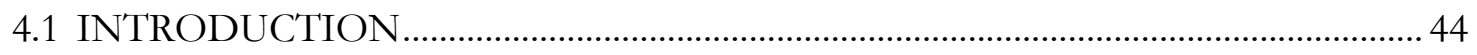

4.2 RECONSTRUCTION ALGORITHM FOR FLUORESCENCE TOMOGRAPHY

4.3 INSTRUMENTATION FOR FLUORESCENCE TOMOGRAPHY ......................... 49

4.4 EXPERIMENTAL METHODS OF FLUORESCENCE TOMOGRAPHY ............ 52 
CHAPTER 5 PAST WORK: CLINICAL DIFFUSE OPTICAL IMAGING IN HUMAN

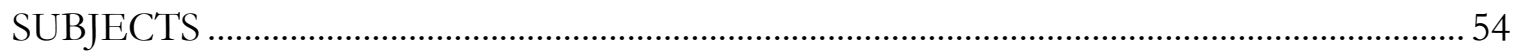

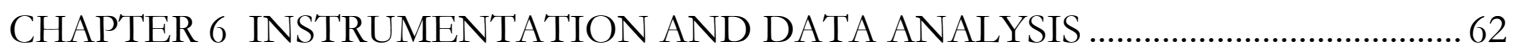

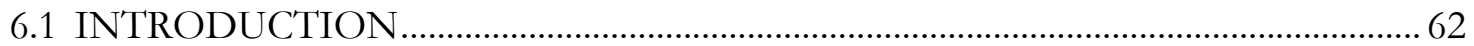

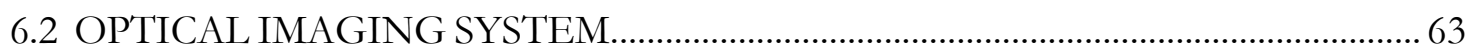

6.3. ACQUISITION OF OPTICAL DATA WITH THE HAND-HELD IMAGER...... 67

6.4. ELIMINATION OF EXCITATION LIGHT LEAKAGE (SUBTRACTION

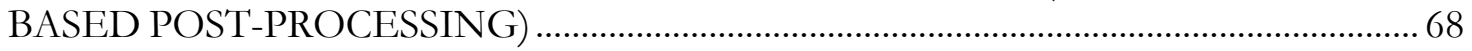

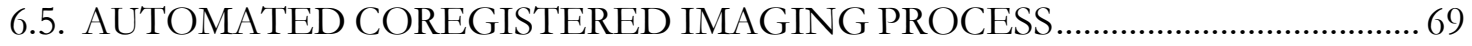

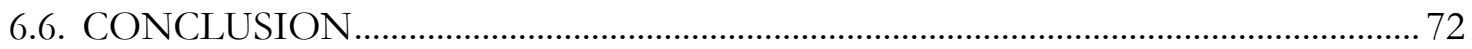

CHAPTER 7 FLUORESCENCE-ENHANCED DIFFUSE OPTICAL TOMOGRAPHY

73

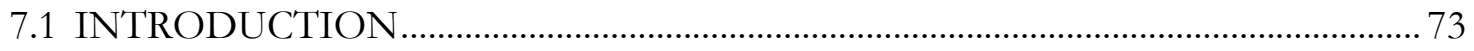

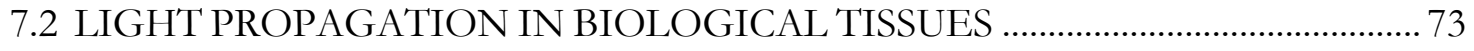

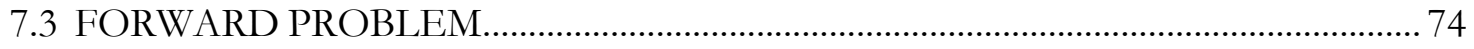

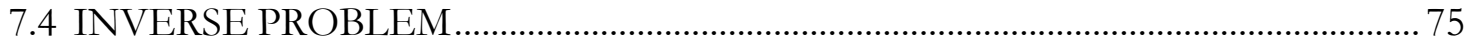

7.5 APPROXIMATE EXTENDED KALMAN FILTER (AEKF) BASED

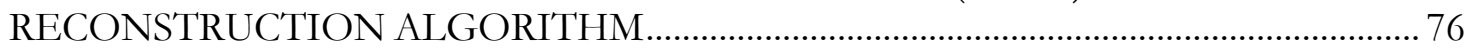

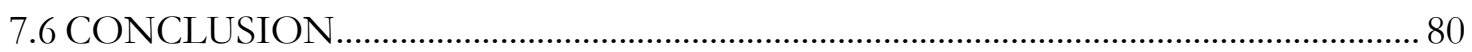

CHAPTER 8 STUDY I: 2D IMAGING IN TISSUE PHANTOMS AND IN VITRO IN

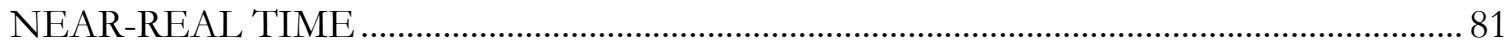

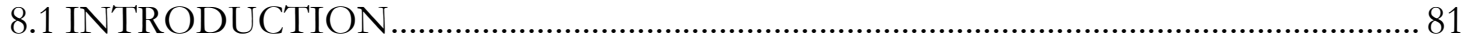

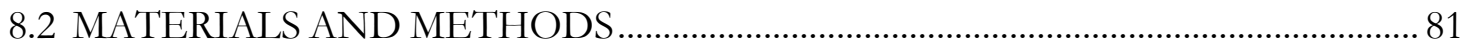

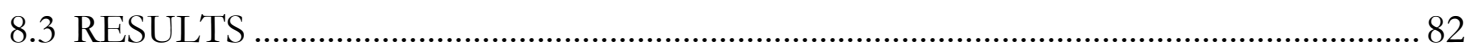

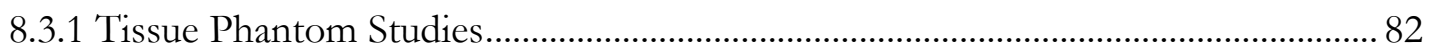

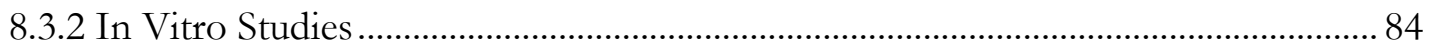

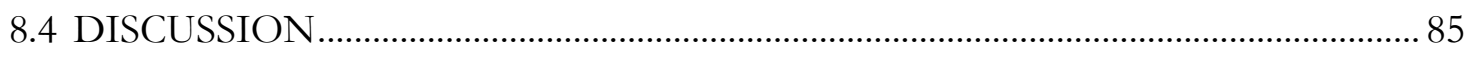

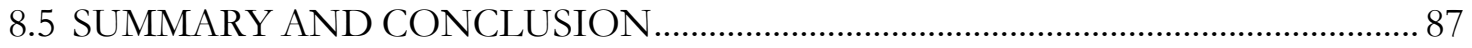

CHAPTER 9 STUDY II: COREGISTERED IMAGING TOWARDS IMPROVED TARGET DEPTH DETECTION (USING MULTIPLE-SCAN COREGISTERED

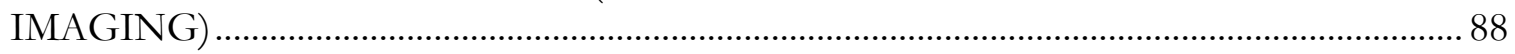

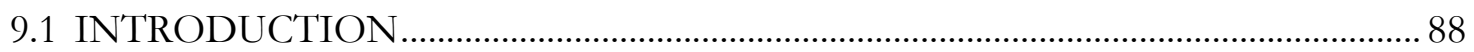

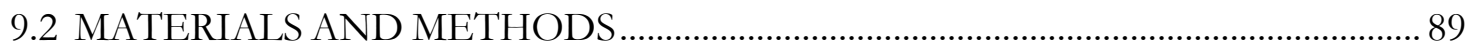

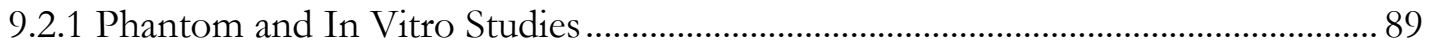

9.2.2 Validation of Coregistered Imaging in Phantoms ...................................................... 90

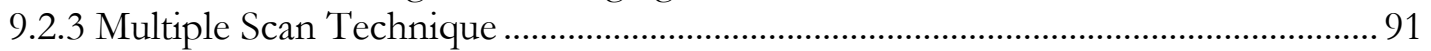

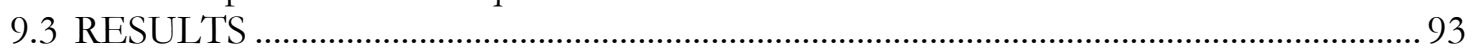

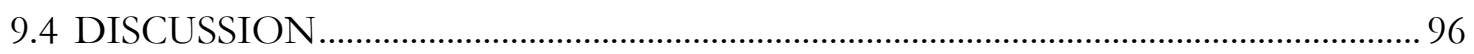

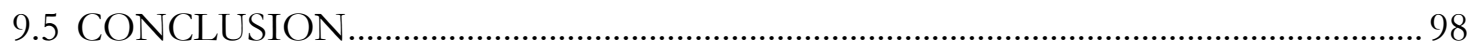

CHAPTER 10 STUDY III: 3D TOMOGRAPHY STUDIES IN TISSUE PHANTOMS AND IN VITRO 
10.1 INTRODUCTION

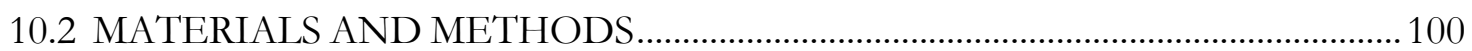

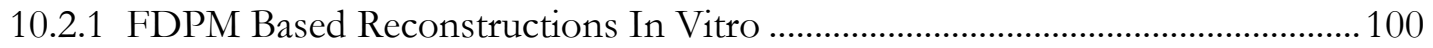

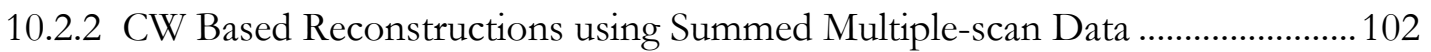

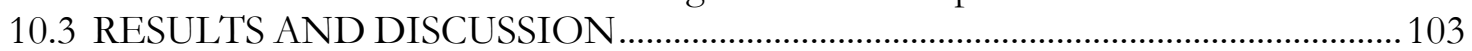

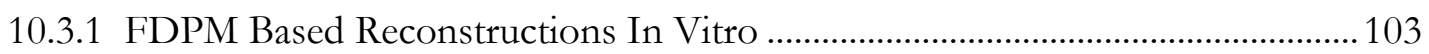

10.3.2 3D CW Based Reconstructions using Summed Multiple-scan Data................... 106

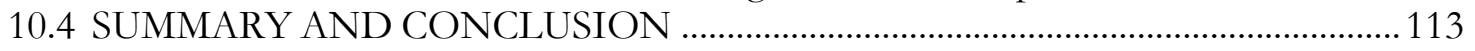

CHAPTER 11 STUDY IV : 2D IMAGING IN VIVO WITH HEALTHY HUMAN SUBJECTS IN NEAR-REAL TIME TOWARDS TARGET DETECTION ....................114

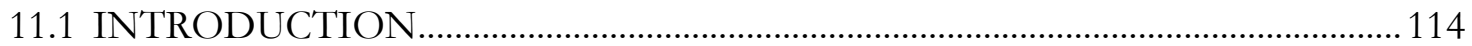

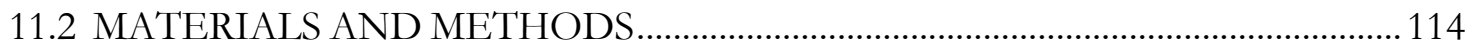

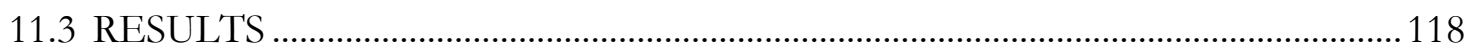

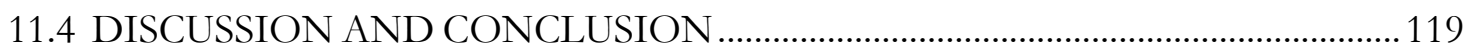

CHAPTER 12 STUDY V: COREGISTERED IMAGING IN VIVO WITH HEALTHY

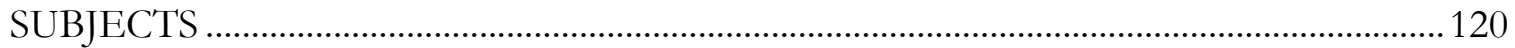

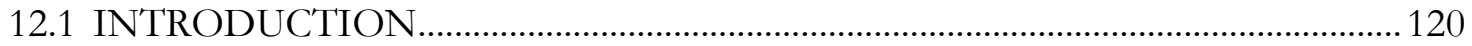

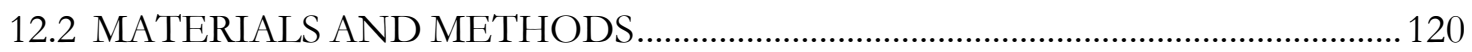

12.2.1 Initial coregistered imaging approach (motorized 3D scanner \& sitting position)

120

12.2.2 Validation of coregistered imaging in human subjects .........................................121

12.2.3. Modification of coregistered imaging in vivo to improve accuracy (hand-held

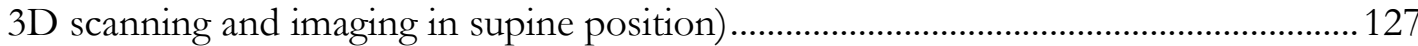

12.2.4 Experimental Study I: Automated Coregistered Imaging using Initial Imaging

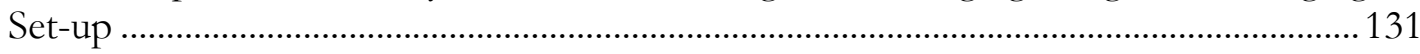

12.2.4 Experimental Study II Automated Coregistered Imaging using the Modified

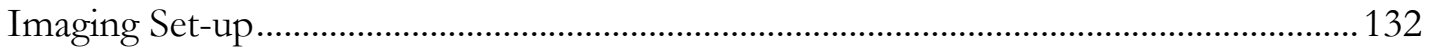

12.2.5 Experimental Study III Manual Coregistered Imaging using the Modified

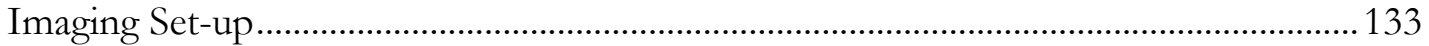

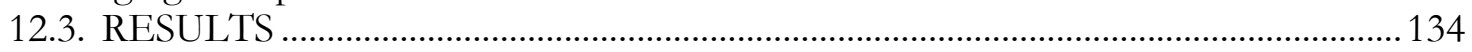

12.3.1 Experimental Study I: Automated Coregistered Imaging using Initial Imaging

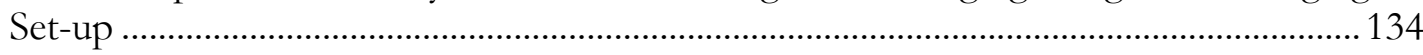

12.3.2 Experimental Study II Automated Coregistered Imaging using the Modified

Imaging Set-up ..................................................................................................................... 135

12.3.3 Experimental Study III Manual Coregistered Imaging using the Modified

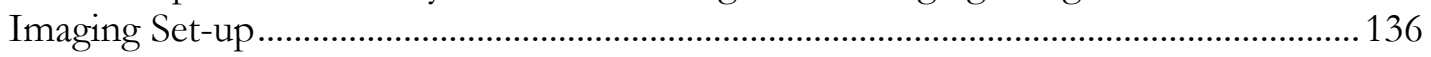

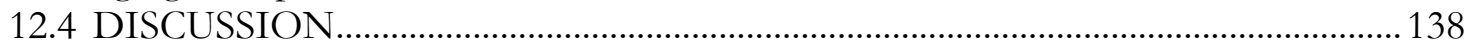

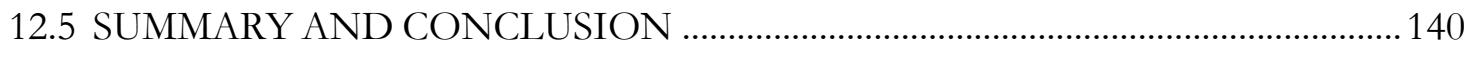

CHAPTER 13 STUDY VI: 3D TOMOGRAPHY IN VIVO WITH A HEALTHY

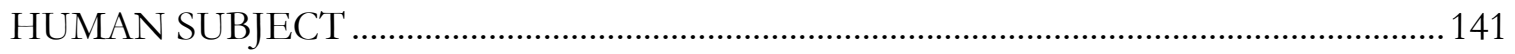

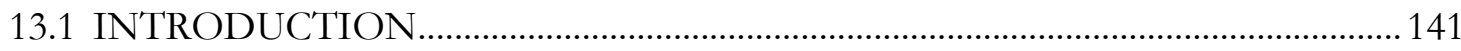

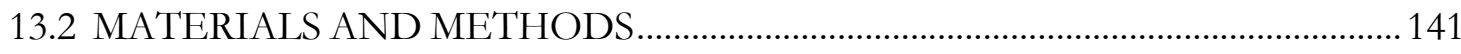

13.2.1 Procedure for 3D Mesh Acquisition and Reconstruction In Vivo.......................141

13.2.2 Fluorescence Tomography in a Healthy Human Subject ...................................... 148 
13.3 RESULTS

13.4 DISCUSSION

CHAPTER 14 STUDY VII: IN VIVO IMAGING OF BREAST CANCER SUBJECTS

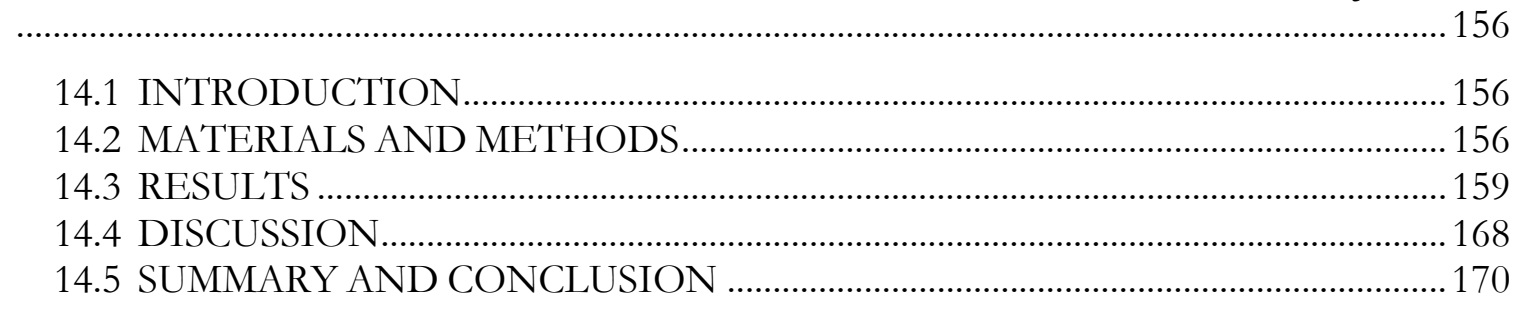

CHAPTER 15 SUMMARY AND CONCLUSION: OVERALL DISCUSSION AND

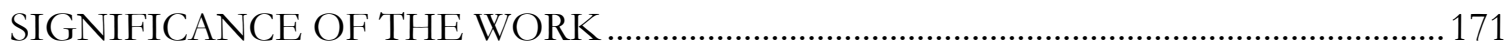

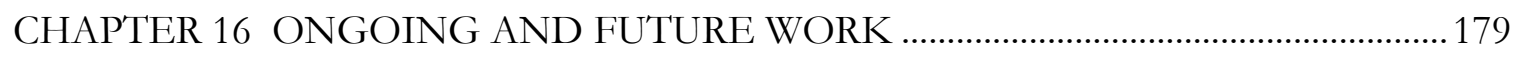

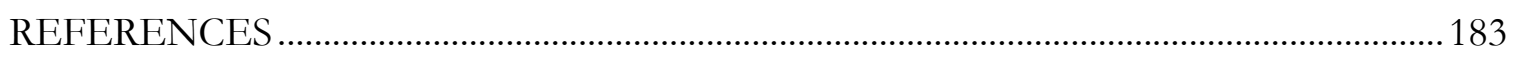

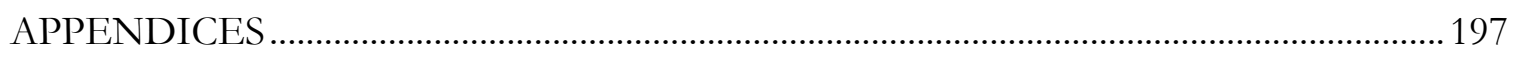

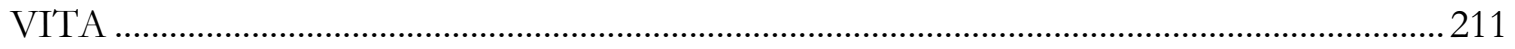




\section{LIST OF TABLES}

TABLE

PAGE

3.1 Summary of different hand-held devices developed towards optical imaging.....

4.1 Summary of the experimental research performed in fluorescence tomography from 2008 to the present..

5.1 Summary of optical properties of healthy breast tissue from the literature.

5.2 Summary of clinical diffuse optical imaging studies between 2008 and 2010 .

7.1 Definitions of terms used in AEKF pseudocode

8.1 Summary of experimental cases for slab tissue phantom and in vitro studies

9.1 Summary of experimental studies in which a target was detected in tissue phantoms and in vitro. The cases where the deepest target was detected for phantom or in vitro, and perfect or imperfect uptake are highlighted in red

9.2 Average and standard deviation of $\mathrm{x}, \mathrm{y}, \mathrm{z}$ coordinates as displayed in tracking software and total distance off between true and measured values. Values are in units of centimeters and each measured value represents an average of 10 measurements while probe was at each of the 4 positions.

10.1 Sample optical property measurements of the in vitro phantom. All optical property values are in units of $\mathrm{cm}-1$

10.2 Summary of the experiment parameters for various experimental cases including the average measured source intensities, the gain setting on the image intensifier, the actual target location in $\mathrm{cm}$, the actual target volume, the measured optical property values of the phantom at the excitation and emission wavelengths, the target to background ratio (T:B), the recovered target location in $\mathrm{cm}$, the recovered $\mu$ axf values in $\mathrm{cm}-1$, the recovered target volume, the number of iterations executed before convergence, and the breakpoint (cutoff value selected on the first breakpoint of a histogram plot of the recovered $\mu$ axf values). Results from experimental cases 26 and 27 (highlighted in yellow) are presented in Section 10.3. 
10.3 Summary of selected experimental cases for summated image

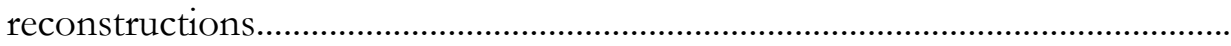

11.1 Summary of experimental cases performed for in vivo fast $2 \mathrm{D}$ imaging studies.

12.1 Average and standard deviation of $\mathrm{x}, \mathrm{y}, \mathrm{z}$ coordinates as displayed in tracking software and total distance off between true and measured values. Values are in units of centimeters and each measured value represents an average of 10 measurements while probe was at each of the 4 positions.

13.1 Quantitative results for the in vivo 3D reconstruction: recovered $\mu_{\text {axf }}$, true target volume, recovered target volume, approximate true target location, recovered target location, total distance off 


\section{LIST OF FIGURES}

FIGURE

PAGE

1.1 Mortality rate by year per 100,000 women with breast cancer............................. 2

1.2 Diagram summarizing the systematic experimental study design...................... 5

1.3 Flow diagram showing the organization of the dissertation................................ 7

2.1 Absorption coefficient at various wavelengths for water, oxy-hemoglobin,

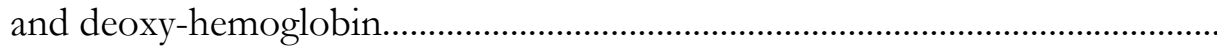

2.2 Jablonski diagram showing the principle of fluorescence.................................... 10

2.3 Principle of fluorescence enhanced optical imaging using continuous-wave based measurements.................................................................................................

2.4 Diagram of the three measurement techniques for optical imaging: (a) continuous wave, (b) time domain photon migration, and (c) frequency domain photon migration.

2.5 Different imaging configurations: (a) compressed tissue, (b) circular, and (c) sub-surface.

2.6 Different types of instrumentation used for breast include: (A) bulky optical bed based imaging, and (B) hand-held device based imaging.............................

2.7 Types of sources commonly used in diffuse optical imaging: (A) laser diode (B) light emitting diode (C) halogen lamp (D) Ti:sapphire tunable laser (E) helium-neon He-Ne long coherence laser.....

2.8 Types of detectors commonly used in diffuse optical imaging: (A) CCD camera (B) avalanche photodiode (C) photomultiplier tube.... 
3.1 Schematics of spectroscopic imaging-based devices. (a) Device \#1a developed by researchers at the University of California, Irvine, which contains a single FD source-detector pair, (b) Device \#1b developed by the same group, which contains a single FD source-detector pair and a single CW source-detector pair, and (c) Device \#2 developed by other researchers at the same university, which contains a single source and detector embedded within the probe, (d) Device \#3 developed by researchers at the University of Pennsylvania, which contains a single source surrounded by 8 detectors, (e) Device \#6a developed by researchers at Ohio State University and ViOptix, Inc., which contains 8 sources and 8 detectors, (e) Device \#7 developed at the University of Pennsylvania, which contains 4 sources and 4 detectors, (f) Device \#8 developed at Physikalisch-Technische Bundesanstalt, which contains 2 sources and 4 detectors, (g) Device \#9a developed at the University of Pennsylvania, which contains 2 sources and 4 detectors, and (h) Device \#9b developed by the same group, which contains 4 sources and 2 detectors.

3.2 Schematics of tomographic imaging-based devices. (a) Device \#10a developed by researchers at the University of Pennsylvania and University of Connecticut, which contains a central ultrasound component surrounded by 12 sources and 4 detectors, (b) Device \#10b developed at the University of Connecticut, which contains an additional 4 detectors, and (c) Device \#11 developed by the same group, which contains an ultrasound component with 12 sources and 8 detectors.

3.3 Schematics of interference imaging-based devices. (a) Device \#4 developed at the University of Pennsylvania, which contains a detector centered between two anti-phase sources for phase cancellation technique, (b) Device \#5 developed by researchers at the Huazhong University of Science and Technology, which contains a detector centered between a pair of inphase sources and a pair of anti-phase which also uses the phase cancellation technique.

6.1 The actual hand-held probe face showing the source-detector configuration. The large red dots represent the six source fiber locations and all other small holes are the 165 detector fiber locations......

6.2 Hand-held probe based optical imaging system showing the hand-held probe is fiber-optically coupled to the laser source and ICCD camera (left). The probe face is flexible to contour to different tissue curvatures (right)

6.3 Schematic of the instrumentation for the hand-held optical imager. The $\mathrm{CW}$ based imaging system is shown in white and the additional components for FDPM based imaging are highlighted in blue..... 
6.4 Experimental set-up for tissue phantom, in vitro, and in vivo studies: (A) empty phantom showing target inclusion and placement of probe, (B) tissue slab phantom with 1\% Liposyn solution for uniform scattering in the background, and $(\mathrm{C})$ in vitro slab phantom with heterogeneous scattering in the background.

6.5 Flowchart of the coregistered imaging process, implemented as automated coregistration software during optical imaging studies

7.1 Illustration of the forward and inverse problem in optical tomography. In the forward problem, the optical property distribution is known and used to predict the measurements that would occur at the surface of the tissue. In the inverse problem, the measurements obtained experimentally at the surface are used to reconstruct the unknown optical property distribution within the tissue.

7.2 Flow diagram of the AEKF based reconstruction algorithm

8.1 Near real-time images of fluorescence intensity obtained as 2D surface contour plots, acquired from slab phantoms (with uniform background scattering). The fluorescent target was placed at different locations and depths: (A) target location: $(\mathrm{x}, \mathrm{y}, \mathrm{z})=(2.0,2.7 .1 .5)$, and $(\mathrm{B})$ target location $(\mathrm{x}, \mathrm{y}, \mathrm{z})=(2.0,2.7,2.0)$ and $(\mathrm{C})$ target location: $(\mathrm{x}, \mathrm{y}, \mathrm{z})=(3.0,2.2,2.5)$. The black hollow circle in each sub-plot is the true target location.

8.2 Fast subtracted images of fluorescence intensity obtained as 2D surface contour plots, acquired from slab phantoms (with uniform background scattering). The $0.45 \mathrm{cc}$ fluorescent target was placed at different locations and depths: (A) target location: $(\mathrm{x}, \mathrm{y}, \mathrm{z})=(2.0,2.7 .1 .5)$, and (B) target location $(\mathrm{x}, \mathrm{y}, \mathrm{z})=(2.0,2.7,2.0)$ and $(\mathrm{C})$ target location: $(\mathrm{x}, \mathrm{y}, \mathrm{z})=(3.0,2.2$, 2.5). The black hollow circle in each sub-plot is the true target location.

8.3 Near-real time images of fluorescence intensity obtained as 2D surface contour plots, acquired from in-vitro slab phantoms (with non-uniform background scattering). The $0.45 \mathrm{cc}$ fluorescent target was located at a depth of (A) $1.0 \mathrm{~cm}$, and (B) $1.5 \mathrm{~cm}$, from the imaging surface. The black hollow circle in each sub-plot is the true target location.

9.1 Experimental set-up for phantom studies. (A) A spherical target filled with $1 \mu \mathrm{M}$ indocyanine green is enclosed within the cubical phantom to represent a tumor. (B) The phantom is composed of a $1 \%$ Liposyn solution to mimic the optical properties of human breast tissue. 
9.2 Coregistered images from single scans (2D contour plots of fluorescence intensity data) at four probe positions for experimental case \#12 (a 0.45 $\mathrm{cm} 3$ fluorescent target placed $3.0 \mathrm{~cm}$ deep, $\mathrm{x}$-dimension in-vitro phantom under $\mathrm{T}: \mathrm{B}=1: 0)$. In each image, the white dotted line represents the probe position with respect to the phantom and the black open circle represents the true target location.

9.3 Summated image of multiple scans shown in Figure 9.2 (experimental case \#12). The summed image represents summation of 8 single scans, where 2 scans were collected at each of the 4 probe positions shown in Figure 9.3. The black open circle represents the true target location.

9.4 Summed images of multiple coregistered scans from the four best experimental cases listed in Table 1. The black open circle indicates the true target location for each case.

10.1 Reconstruction of $2.0 \mathrm{~cm}$ deep target within heterogeneous in vitro phantom under perfect uptake case. (a) 2D image collected using handheld device, (b) 3D view of isosurface plot of reconstructed target, (c) three views of reconstructed target (solid open circle represents true target location; dotted line encircles recovered target). The term V1 in the legend represents the scale for the recovered $\mu$ axf values in $\mathrm{cm}^{-1}$.....

10.2 Reconstruction of $2.5 \mathrm{~cm}$ deep target within heterogeneous in vitro phantom under perfect uptake case. (a) 2D image collected using handheld device, (b) 3D view of isosurface plot of reconstructed target, (c) three views of reconstructed target (solid open circle represents true target location; dotted line encircles recovered target). The term V1 in the legend represents the scale for the recovered $\mu$ axf values in $\mathrm{cm}^{-1}$.

10.3 3D reconstruction using summed coregistered data. (A) 2D contour plot of summed images collected using hand-held probe. (B) Coregistered image of summed data with phantom mesh. (C) Isosurface plot of 3D reconstruction. (D) Contour slices showing intensity data of 3D reconstruction. The true target location is indicated by a black circle (A-B) or a dark red sphere (C-D)

10.4 Individual 3D reconstructions (shown as contour slices) of the 8 single scans from experimental case A in Table 10.3. The grey sphere represents the true target location.

10.5 Results for the first method of CW based summated multiple-scan reconstruction (shown as contour slices) where the recovered $\mu$ axf values from the individual reconstructed scans were summed together. The true target location in each plot is indicated by a grey sphere. 
10.6 Results for 3D reconstructions performed using multiple summated coregistered images. Details of each experimental cases are given in Table 10.3. The yellow dotted circles indicate possible recovered signal from the fluorescent targets. The true target locations are indicated by grey spheres circled in black for emphasis.

11.1 Schematic showing the location of an absorbing target placed superficially underneath the breast tissue and the location of the probe during imaging...

11.2 Experimental set-up for in vivo studies with human subject (mannequin shown here for demonstration only). The hand-held probe is placed in full contact with the breast tissue. The raw image and the $2 \mathrm{D}$ contour plot are displayed on the computer screen.

11.3 Systematic grid developed for future imaging studies. The positions are measured with reference to the center (nipple) region which is indicated by the red dotted circle.

11.4 Fast subtracted images of fluorescence intensity obtained as $2 \mathrm{D}$ surface contour plots, acquired in-vivo from a human subject using a spherical fluorescent target, for two experimental cases: (A) the probe was in the flat position and a $0.23 \mathrm{cc}$ target was placed at the 4 o'clock position, and (B) the probe was in the curved position and a $0.45 \mathrm{cc}$ target was placed at 8 o'clock position. The images acquired using the probe in the curved position are illustrated as projected as a flat $2 \mathrm{D}$ image, in order to be consistent with the images presented in case (A) (i.e. using the probe in flat position)

11.5 Fast subtracted image of fluorescence intensity obtained as 2D surface contour plot, acquired in-vivo from a human subject using two spherical fluorescent targets $(0.23$ and $0.45 \mathrm{cc})$.

12.1 Initial set-up for acquiring 3D breast tissue geometry for coregistered imaging..

12.2 Automated input of reference points within any 3D scanned geometry.........

12.3 Placement of reference points on subject and initialization of coregistration software

12.4 Screen capture of coregistration software showing meshed geometry of breast tissue with reference points (probe location inactive in this view).

12.5 Images from coregistration showing probe position tracked in real-time at different reference locations. 
12.6 Coregistered image of simulated target behind human breast tissue. White dotted line represents the probe position

12.7 Modified human subject imaging set-up. Subject lies supine in recliner chair $\left(45^{\circ}\right)$ during acquisition of tissue geometry with 3D scanner and imaging using handheld imager.

12.8 'Clock' method for referencing locations on the breast tissue. The relative section of the tissue is determined by the position on the 'clock' (shown on left side) and the distance from the nipple is measured in centimeters (shown on right side).

12.9 Modified imaging set-up. The subject lies in supine position in recliner chair while breast tissue is imaged using hand-held optical imager. Mannequin shown for demonstration only

12.10 Geometry acquired using the modified imaging set-up with a healthy subject lying supine in recliner chair with $45^{\circ}$ angle. The white dotted line represents the probe location during imaging. (A) Frontal view. (B) Side view.....

12.11 Coregistered images of fluorescence intensity data collected form a normal subject with an ICG-filled target using the initial set-up (subject seated in upright position). (A) Location of probe and image relative to the subject. (B) Case\#1 image: $0.45 \mathrm{~cm} 3$ target at the 6 o'clock position. (C) Zoomed image of Case \#1. (D) Case \#2 image: $0.45 \mathrm{~cm} 3$ target at the 4 o'clock position. (E) Case \#3 image: $0.23 \mathrm{~cm} 3$ target at the 8 o'clock position. All targets contain $1 \mu \mathrm{M}$ indocyanine green

12.12 Automated coregistered images in normal subject (no target) using modified imaging set-up. (A) Probe position \#1. (B) Frontal view of coregistered image at probe position \#1. (C) Side view of coregistered image at probe position \#1. (D) Probe position \#2. (E) Frontal view of coregistered image at probe position \#2. (F) Side view of coregistered image at probe position \#2.

12.13 Manually coregistered images from normal subject with $0.45 \mathrm{~cm} 3$ target with $0.08 \%$ India ink placed under the breast tissue at the 6 o'clock position.

12.14 Manually coregistered images from normal subject with $0.45 \mathrm{~cm} 3$ target with $0.02 \%$ India ink placed under the breast tissue at the 6 o'clock position.

13.1 Scanned surface geometry of breast tissue. The imaged section was cut from the full geometry and used in the reconstruction. 
13.2 Volume geometry of scanned breast tissue.

13.3 Illustration of the two methods of assigning node numbers. (A) The mesh generated by MATLAB and exported from the coregistration software assigns the elements first and then gives each node in the element number assignments independent of the adjacent elements resulting in multiple numbers assigned to the same node. (B) The preferred method is where the node numbers are assigned first and then elements are constructed among those nodes such that each node is shared by multiple elements and only assigned a single number resulting in much fewer nodes than elements

13.4 Automated coregistered image of a fluorescent target placed beneath the breast tissue in a normal subject which was used to perform the 3D reconstruction.

13.5 (A) Plot of the 3D tetrahedral unstructured mesh used in the reconstruction. (B) Side view of contour slices in the x-plane...........................

13.6 Contour slices in the x-plane at $0.25 \mathrm{~cm}$ intervals. Slice depth is indicated as depth from the tissue surface at the center (nipple) region....

13.7 Contour slices in the y-plane at $0.25 \mathrm{~cm}$ intervals

14.1 Schematic showing the probe locations during the imaging studies. The probe was placed at four different locations on the left breast tissue followed by the same four locations on the right breast tissue

14.2 Probe locations for second set of images in subject \#2. The subject was asked to lie on the right side and rest the left arm above the head while the probe was placed at four different locations on the outer side of the left breast starting under the arm and moving down toward the ribcage. The procedure was repeated on the right breast at symmetric locations. All probe positions were estimated with reference to the nipple location and are not drawn to scale in the schematic.

14.3 Three slices of an MRI scan from 64 year-old breast cancer patient. The yellow arrows indicate the location of abnormal tissue behind the nipple region 
14.4 Continuous-wave absorption-based optical images collected from subject \#1 at four probe locations (first repetition of images). The images on the left were collected from the left breast tissue and the images in the center were collected from the right breast tissue. The post-processed images on the right represent the normalized intensity data from the ipsilateral (left) breast subtracted from the normalized intensity data from the contralateral (right) breast showing the optical signal due to absorption as a higher (red)

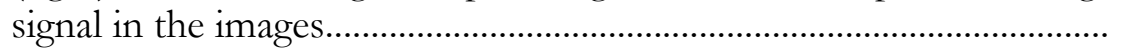

14.5 Continuous-wave absorption-based optical images collected from subject \#1 at four probe locations (second repetition of images). The images on the left were collected from the left breast tissue and the images in the center were collected from the right breast tissue. The post-processed images on the right represent the normalized intensity data from the ipsilateral (left) breast subtracted from the normalized intensity data from the contralateral (right) breast showing the optical signal due to absorption as a higher (red) signal in the images.

14.6 Ultrasound images from subject \#1 of suspicious enlarged axillary lymph nodes. (The axillary region is indicated in Figure 14.2.)....................................

14.7 Continuous-wave absorption-based optical images collected from subject $\# 1$ at the second set of four probe locations (repetition \#1). The images on the left were collected from the left breast tissue and the images in the center were collected from the right breast tissue. The post-processed (subtracted) images on the right show the optical signal due to absorption. The yellow dotted circle was hand-drawn to represent the region of interest in each image......

14.8 Continuous-wave absorption-based optical images collected from subject \#1 at the second set of four probe locations (repetition \#2). The images on the left were collected from the left breast tissue and the images in the center were collected from the right breast tissue. The post-processed (subtracted) images on the right show the optical signal due to absorption. The yellow dotted circle was hand-drawn to represent the region of interest in each image

14.9 X-ray mammography images of the left breast from a 51 year-old breast cancer patient. (A) The top of the image is the left side and the bottom of the image is the right side of the breast tissue. (B) Oblique image where the top of the image is toward the patient's head and left side and the bottom of the image is toward the patient's feet and right side. The yellow arrows indicate 3 lesions (labeled as L1, L2, and L3) located in the 1 o'clock to 2 o'clock region of the breast. 
14.10 Ultrasound images of the left breast from a 51 year-old breast cancer patient. Both images were collected in the 1 to 2 o'clock location where image (A) was $2-3 \mathrm{~cm}$ from the nipple and image (B) was $6 \mathrm{~cm}$ from the nipple. The yellow arrows indicate 3 lesions (labeled as L1, L2, ans L3 corresponding to Figure 14.7) located in the 1 o'clock to 2 o'clock region of

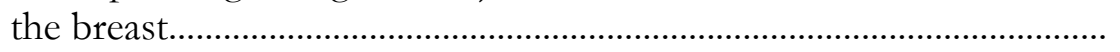

14.11 Optical images collected from the breast tissue of a 51 year-old breast cancer patient. The images in Set \#1 were collected from the ipsilateral breast and the images in Set \#2 were collected from the contralateral breast. The images from Set \#1 were subtracted from Set \#2 so that the area of higher absorption shows as a higher (or more red) signal in the postprocessed images. The yellow dotted circle was hand-drawn to indicate the region of interest

14.12 Comparison of the results from two different image sets collected on different dates from subject \#2. Study \#2 was performed 42 days after Study \#1 in the same breast cancer subject. Four images were collected at the same probe locations (see Figure 14.1). For each study, images were collected from both the right and left breast tissues and used to subtract the excitation leakage. All images are the final post-processed images. The probe locations were estimated with respect to the nipple location 


\section{LIST OF SYMBOLS}

\begin{tabular}{|c|c|}
\hline$\mu_{\mathrm{a}}$ & Absorption coefficient \\
\hline$\mu_{\mathrm{s}}$ & Scattering coefficient \\
\hline$\mu_{\mathrm{s}}{ }^{\prime}$ & Reduced scattering coefficient \\
\hline $\mathrm{H}_{2} \mathrm{O}$ & Water \\
\hline $\mathrm{HbO}_{2}$ & Oxy-hemoglobin \\
\hline $\mathrm{Hb}$ & Deoxy-hemoglobin \\
\hline $\mathrm{HbT}$ & Total Hemoglobin \\
\hline $\mathrm{SO}_{2}$ & Oxygen saturation \\
\hline$\mu \mathrm{M}$ & Micro-moles \\
\hline$\square$ & Phase \\
\hline$x$ & Excitation \\
\hline$m$ & Emission \\
\hline$\square$ & Fluence \\
\hline$q$ & Quantum efficiency \\
\hline$\square$ & Fluorescence lifetime \\
\hline$c$ & Speed of light \\
\hline$\square$ & Angular frequency \\
\hline $\mathbf{r}$ & Positional vector \\
\hline$g$ & Anisotropy factor \\
\hline$\square$ & Index mismatch parameter \\
\hline$n$ & Surface normal \\
\hline$\square_{\mathrm{i}}$ & Fluence (at location i) \\
\hline $\mathbf{x}$ & Distribution of predicted measurements (of parameter of interest) \\
\hline $\mathbf{z}$ & Experimental measurements (corresponding to predicted measurements) \\
\hline $\mathbf{y}$ & Unknown parameter (optical property in tissue) \\
\hline f & Forward simulator \\
\hline$\mu_{\text {axi }}$ & Absorption coefficient of the tissue chromophores \\
\hline$\mu_{\mathrm{axf}}$ & Absorption coefficient of the fluorophore \\
\hline $\mathrm{J}$ & Jacobian matrix \\
\hline Q & Model error covariance \\
\hline $\mathrm{K}$ & Gain matrix \\
\hline $\mathrm{R}$ & Measurement error covariance \\
\hline $\mathrm{P}$ & Parameter error covariance \\
\hline
\end{tabular}




\section{LIST OF ACRONYMS}

$\begin{array}{ll}\text { MRI } & \text { Magnetic Resonance Imaging } \\ \text { PEM } & \text { Positron Emission Tomography } \\ \text { BSGI } & \text { Breast Specific Gamma Imaging } \\ \text { NIR } & \text { Near-Infrared } \\ \text { 3D } & \text { Three-Dimensional } \\ \text { 2D } & \text { Two-Dimensional } \\ \text { nm } & \text { nanometers } \\ \text { cm } & \text { centimeters } \\ \text { CT } & \text { Computed Tomography } \\ \text { DOT } & \text { Diffuse Optical Tomography } \\ \text { FDOT } & \text { Fluorescence-enhanced DOT } \\ \text { ICG } & \text { Indocyanine Green } \\ \text { FDA } & \text { Food and Drug Administration } \\ \text { CW } & \text { Continuous Wave } \\ \text { FDPM } & \text { Frequency-Domain Photon Migration } \\ \text { DC } & \text { Direct Current } \\ \text { CCD } & \text { Charge-Coupled Device } \\ \text { ICCD } & \text { Intensified CCD } \\ \text { APD } & \text { Avalanche Photo-Diode } \\ \text { PMT } & \text { Photo-Multiplier Tube } \\ \text { FMT } & \text { Fluorescence Molecular Tomography } \\ \text { PCA } & \text { Principle Component Analysis } \\ \text { FT } & \text { Fluorescence Tomography } \\ \text { XCT } & \text { X-ray Computed Tomography } \\ \text { THC } & \text { Total Hemoglobin Concentration } \\ \text { DOS } & \text { Diffuse Optical Spectroscopy } \\ \text { ROI } & \text { Region of Interest } \\ \text { SP } & \text { Scattering Power } \\ \text { AC } & \text { Alternating Current } \\ \text { MCP } & \text { Micro-Channel Plate } \\ \text { AEKF } & \text { Approximate Extended Kalman Filter } \\ \text { RMSE } & \text { Root Mean Square Error } \\ & \end{array}$




\section{CHAPTER 1}

\section{Introduction}

Clinical imaging of breast tissue holds an essential role in cancer detection, staging ${ }^{1}$, treatment monitoring, and surgical guidance. Early diagnosis of breast cancer by medical imaging technology is integral for reducing the mortality ${ }^{2}$ and morbidity ${ }^{3}$ of the disease. Breast cancer is the second leading cause of death in women in the U.S. and one out of every eight women are diagnosed with the disease each year.[1] Figure 1.1 shows the mortality rate by year per 100,000 women with breast cancer from different regions of the world. The figure shows that the mortality rate due to breast cancer has declined since the early 1990 s (shaded yellow region), and this is largely due to early diagnosis with improved imaging technology.

Current screening and diagnostic modalities used in the clinical setting include x-ray mammography, breast ultrasound, nuclear imaging, and magnetic resonance imaging (MRI). Combinations of these modalities are used complementarily to noninvasively detect the presence of lesions and determine if they are benign or malignant. However, these methods are neither comprehensive nor infallible leading to unnecessary biopsies and undetected cancers. X-ray mammography has good resolution, however it requires painful compression of the breast tissue, it uses harmful ionizing radiation, and the sensitivity decreases in women with denser breast tissue. Breast ultrasound is nonionizing, portable, and inexpensive, but it has poor imaging quality and contrast. MRI is nonionizing, has good resolution and good

\footnotetext{
${ }^{1}$ Determination of the degree to which a cancer has spread

2 The rate of death due to a disease

${ }^{3}$ The rate of incidence of a disease
} 
sensitivity in dense breast tissue, however it requires expensive, bulky instrumentation and strong magnetic fields. Nuclear imaging methods, including positron emission mammography (PEM) and breast specific gamma imaging (BSGI) provide functional information and can detect small tumors, but they require harmful nuclear radiation.

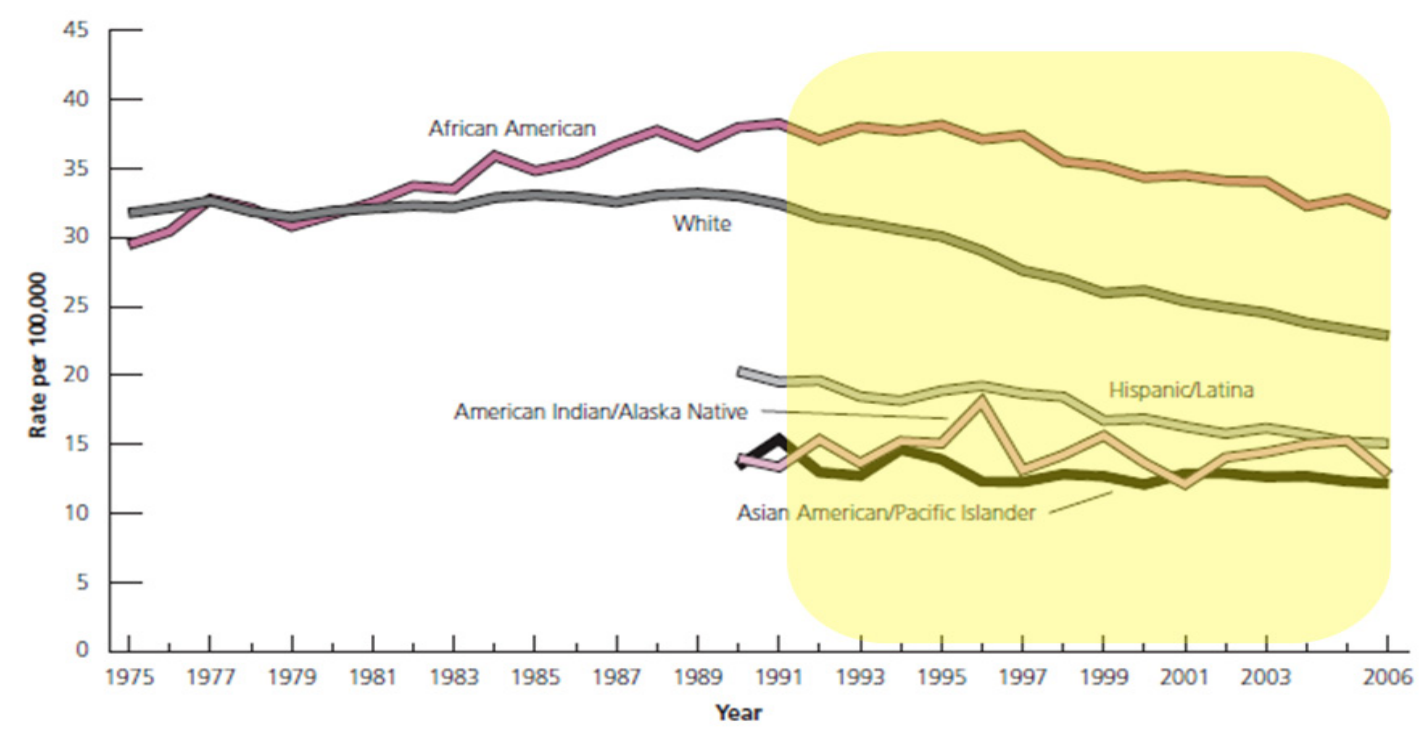

*Rates are age-adjusted to the 2000 US standard population.

Data source: National Center for Health Statistics, Centers for Disease Control and Prevention, 2009. For Hispanics, information is included for all states except Connecticut, Louisiana, Maine, Maryland, Minnesota, New Hampshire, New York, North Dakota, Oklahoma, Vermont, and Virginia and the District of Columbia.

Figure 1.1 Mortality rate by year per 100,000 women with breast cancer. (Adapted from American Cancer Society, Breast Cancer Facts and Figures, 2009)

Currently, x-ray mammography is the gold standard for breast cancer detection. There are two types of $\mathrm{x}$-ray mammograms: (i) screening mammograms are performed annually in women over age 40 or 50 in order to detect the presence of any suspicious mass; (ii) diagnostic mammograms are performed once a mass is detected during the screening process in order to try to gain more information of the size and location, and determine if the lesion is benign or malignant. Diagnostic mammograms are typically followed by breast ultrasound and then MRI and/or nuclear imaging methods. These clinical imaging methods are used to noninvasively determine if a suspicious mass is benign or malignant prior to invasive surgical 
biopsy. However, $80 \%$ of biopsies performed turn out to be negative, resulting in unnecessary physical and psychological trauma for the patient.[2] Hence, there is a clear need for improved breast imaging technology to obviate the number of unnecessary invasive biopsies.

Diffuse optical imaging using near-infrared (NIR) light is a promising technology which has been developed over the past three decades towards applications such as functional brain mapping and breast cancer diagnosis. The method is noninvasive, uses nonionizing radiation, requires relatively inexpensive instrumentation, and provides functional information from in vivo biological tissues. Diffuse optical imaging measures the endogenous optical property difference between normal and diseased tissue (e.g. cancer). External fluorescent contrast agents can be used towards imaging tumors at the molecular stage (fluorescence-enhanced diffuse optical imaging). Diffuse optical imaging has a potential role in the clinical setting as a complementary diagnostic tool for breast cancer. Xray mammography has a 10\% false negative rate [2] (and increases in women with denser breast tissue) which means many cancers go undetected, especially in younger women with denser breasts. Hence, additional imaging tools are required for improved diagnosis.

Several research groups are developing diffuse optical imaging as a complementary imaging modality towards breast cancer diagnosis. Most of the optical imaging systems require bulky instrumentation and/or compression of the breast tissue. To overcome these limitations, hand-held based optical imaging devices are developed, which are portable and patient-comfortable, towards clinical translation of the technology. However, the hand-held devices developed to date have not demonstrated three dimensional (3D) tomography. ${ }^{4}$

\footnotetext{
4 A method of producing a three-dimensional image of the internal structures of an object by the observation and recording of the differences in the effects on the passage of waves of energy impinging on those structures
} 
Currently, a hand-held based optical imaging device has been developed in our Optical Imaging Laboratory, which is capable of 3D tomography, and has been tested in homogeneous ${ }^{5}$ tissue phantoms ${ }^{6}$ [3]. The challenge now is to translate the device to the clinical setting in order to perform 3D tomography on human breast tissue. The overall objective of the dissertation is the clinical translation of our hand-held optical imager toward bedside imaging and 3D tomography of breast cancer. The results will demonstrate for the first time the feasibility of performing 3D tomography in complex human tissues using a hand-held optical imager.

In order to facilitate clinical translation of the hand-held optical imager, a systematic series of experimental studies was designed and executed to demonstrate and evaluate the implementation of (i) two-dimensional (2D) imaging in near-real time (i.e. fast imaging), (ii) coregistered imaging ${ }^{7}$, and (iii) 3D tomography in a clinical setting. To this end, experiments were performed initially in homogeneous tissue phantoms to begin with a simplistic case. Subsequent experiments were then performed in vitro ${ }^{8}$ to better mimic the heterogeneous ${ }^{9}$ nature of biological tissues. The bench-top ${ }^{10}$ imaging set-up was modified into a pseudobedside imaging set-up, and experiments were performed in vivo ${ }^{11}$ with healthy human subjects to determine the performance on actual breast tissues. Finally, preliminary studies

\footnotetext{
${ }^{5}$ Uniform distribution of scattering properties

${ }^{6}$ A physical model designed to mimic the body or part of the body

7 The process of accurately positioning the acquired data at the probe location relative to the breast tissue geometry, which is necessary to perform 3D tomography (details of our coregistered imaging approach are given in subsequent chapters)

${ }^{8}$ Located outside the organism in a laboratory environment

${ }^{9}$ Non-uniform distribution of scattering properties

${ }^{10}$ Suitable in size or configuration for use on a laboratory workbench

${ }^{11}$ Located within the whole, living organism
} 
were performed with cancer patients to test the ability of the device to image breast cancer in vivo. The experimental design is summarized in Figure 1.2.

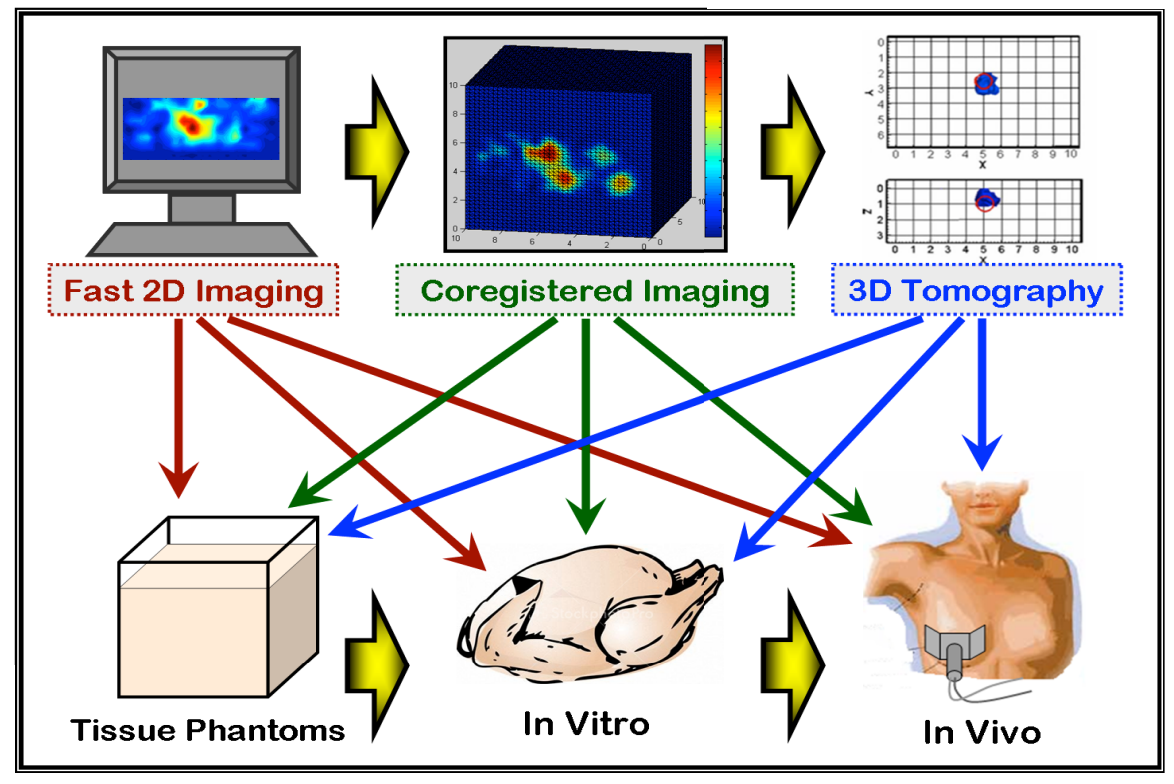

Figure 1.2 Diagram summarizing the systematic experimental study design.

The dissertation is organized as follows: Chapter 2 provides background on nearinfrared optical imaging; Chapter 3 gives a review of the hand-held optical imaging devices developed by different research groups; Chapters 4 and 5 summarize the recent work performed in fluorescence tomography (in phantoms and small animals) and clinical breast imaging studies (in human subjects), respectively; Chapter 6 provides the details of the instrumentation of the hand-held optical imager and describes the procedures for data acquisition, processing, and analysis; Chapter 7 describes the reconstruction method used to perform 3D tomography; Chapters 8-14 detail the experimental studies described above and summarized in Figure 1.2; Chapter 15 gives an overall discussion of the significant results of the studies and Chapter 16 describes ongoing and future work. A flow diagram of the dissertation structure is given in Figure 1.3. 
The various studies are performed using both diffuse optical (i.e. using endogenous ${ }^{12}$ tissue contrast) and fluorescence-enhanced optical imaging (i.e. using an exogenous ${ }^{13}$ contrast agent) towards clinical translation of the hand-held optical imager. The work presented herein will demonstrate the potential role of the hand-held optical imager as a safe and patient-comfortable method to provide complementary physiological ${ }^{14}$, diagnostic and prognostic $^{15}$ information about human breast tissues.

\footnotetext{
12 Originating from inside an organism

13 Originating from outside an organism

14 Relating to the biological function of tissue

15 Predicting the likely outcome or progression of a disease
} 


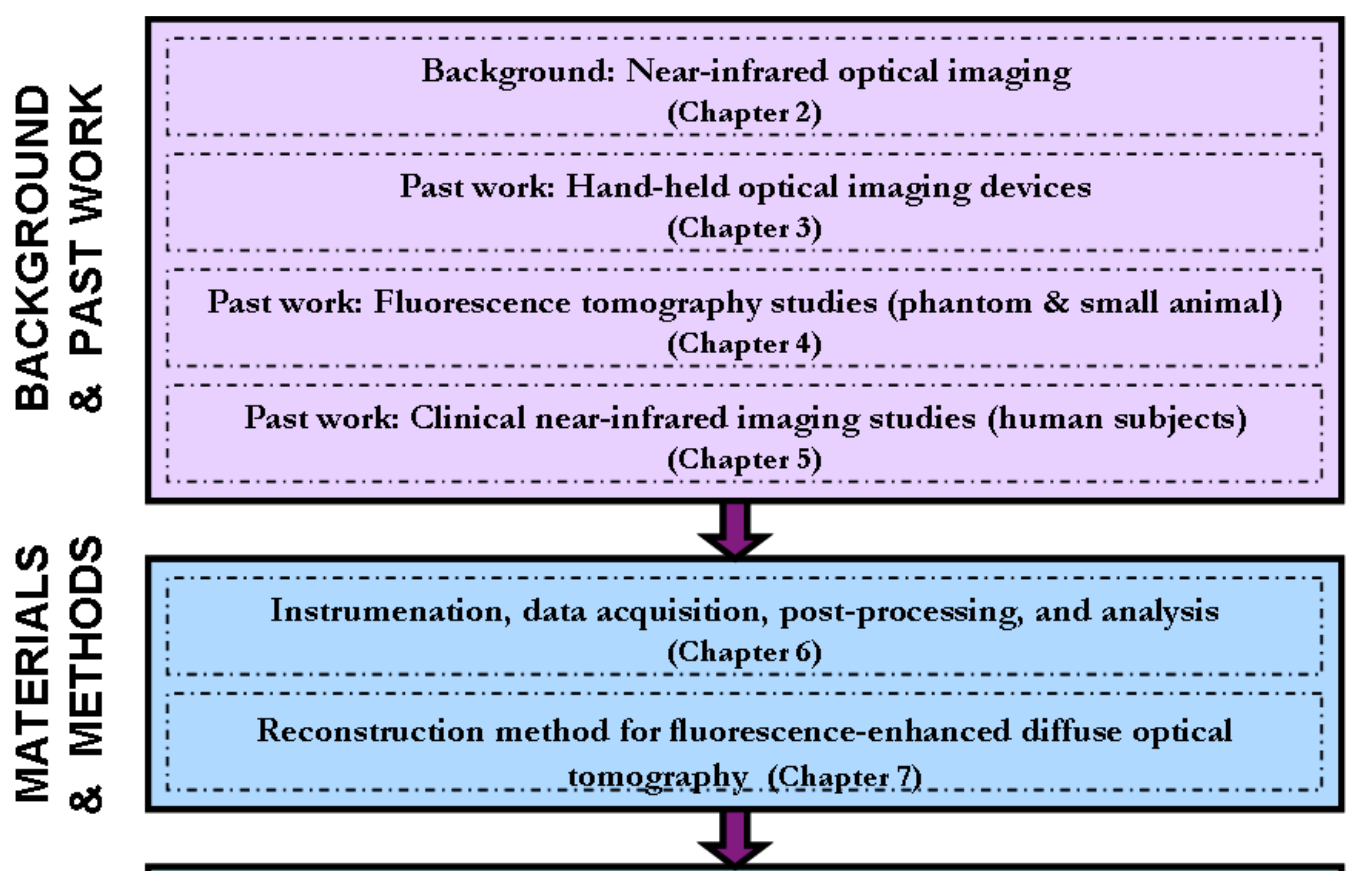

Study I: $2 \mathrm{D}$ imaging in tissue phantoms and in vitro in near real-time (Chapter 8)

Study II: Cotrgistered imaging towards improved target depth detection (Chapter 9)

-

Study III: 3D tomography studies in tissue phantoms and in vitro (Chapter 10)

Study IV: $2 \mathrm{D}$ imaging in vivo with healthy human subjects in near-real time towards target detection_(Chapter 11)

Study V: Cotrgistered imaging in vivo with healthy human subjects

Study V: Cotrgistered imaging in vivo w
(Chapter 12)

Study VI: 3D tomography in vivo with a healthy human subject (Chapter 13)

Study VII: In vivo imaging of breast cancer subjects (Chapter 14)

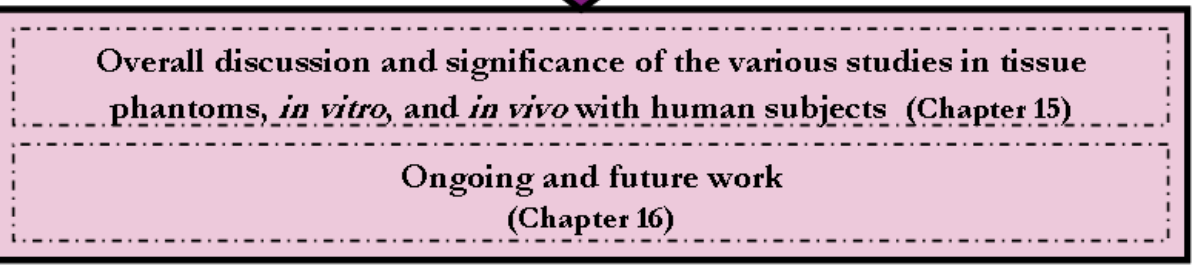

Figure 1.3: Flow diagram showing the organization of the dissertation. 


\section{CHAPTER 2}

\section{Background: NIR Optical Imaging}

\subsection{INTRODUCTION}

Optical imaging is an emerging technique for observing internal functionality of the human body. Three major biomedical applications of optical imaging that have been investigated are brain imaging for functional monitoring, breast imaging for cancer diagnosis and monitoring response to neoadjuvant chemotherapy ${ }^{16}$, and muscle imaging for physiology monitoring [4]. For these applications, diffusing light fields are used to measure blood hemodynamics ${ }^{17}$ and to detect the presence of tissue inhomogeneities [5]. Biological tissues have specific optical properties that become altered in diseased tissue. These altered properties can be discerned by NIR imaging for the application of cancer diagnosis. This chapter will describe tissue characterization using NIR imaging, fluorescence enhanced optical imaging, different measurement techniques and configurations used in optical imaging, and different types of instrumentation used to perform optical imaging.

\subsection{NIR IMAGING FOR TISSUE CHARACTERIZATION}

When light travels through biological tissue, it becomes scattered and absorbed. Biological tissues are characterized by their absorption coefficient, $\mu_{\mathrm{a}}$, and scattering coefficient, $\mu_{\mathrm{s}}$, which represent the number of absorption or scattering events per unit length, respectively, and are wavelength dependent properties intrinsic to the tissue. These properties govern how the light will propagate within that tissue. The major biological chromophores (light absorbers) are water $\left(\mathrm{H}_{2} \mathrm{O}\right)$, oxy-hemoglobin $\left(\mathrm{HbO}_{2}\right)$, and deoxy-hemoglobin $(\mathrm{Hb})$. The

\footnotetext{
16 Preliminary cancer therapy that precedes surgery or a second modality of treatment

17 The branch of physiology that studies the movement of blood
} 
major source of scattering is lipids (fatty tissue). Light is minimally absorbed by the chromophores and preferentially scattered at wavelengths in the near-infrared (NIR) region, 700-900 nanometers $(\mathrm{nm})$ as shown in Figure 2.1. This region is termed the diagnostic or therapeutic window because it is at these wavelengths that light can penetrate deep into the tissue before being absorbed.

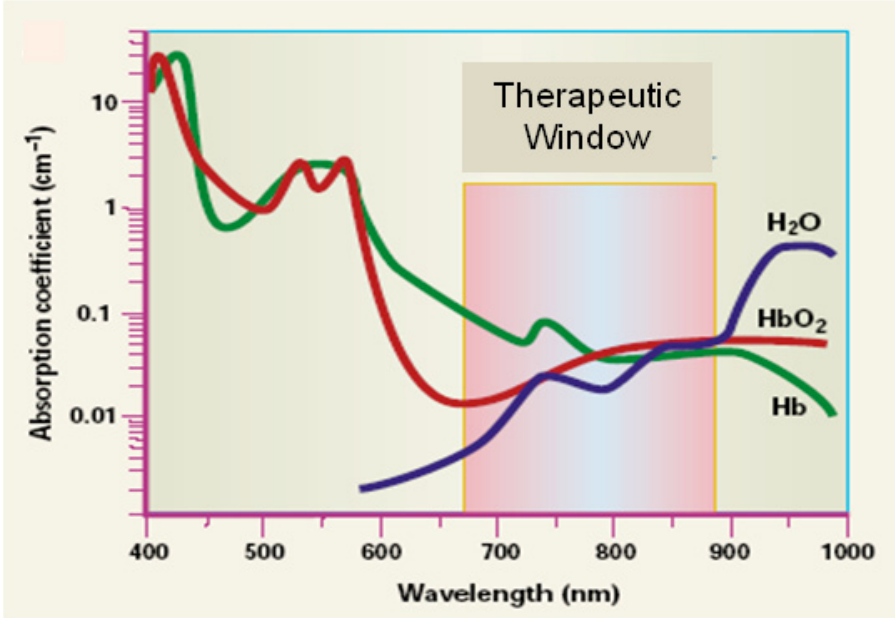

Figure 2.1 Absorption coefficient at various wavelengths for water, oxy-hemoglobin, and deoxyhemoglobin (adapted from R. Weissleder 2001 [6]).

NIR light can be used to detect the presence of inhomogeneities (such as a cyst or tumor) within biological tissue because the optical properties $\left(\mu_{\mathrm{a}}\right.$ and $\left.\mu_{\mathrm{s}}\right)$ differ in normal and diseased tissue. The functional information provided by NIR imaging enables differentiation between benign and malignant lesions, whereas modalities such as x-ray mammography and computed tomography (CT) only provide anatomical information requiring a needle biopsy to identify the inhomogeneity. The endogenous contrast between normal and diseased tissue can be measured by diffuse optical imaging systems to detect the presence of a tumor. 


\subsection{FLUORESCENCE-ENHANCED OPTICAL IMAGING}

An exogenous contrast agent can be introduced into the tissue to produce a stronger signal towards detection of smaller lesions $(<0.5 \mathrm{~cm}$ diameter). Indocyanine green (ICG) is a fluorescing dye typically used for fluorescence-enhanced optical imaging and tomography because it is nontoxic at low concentrations and is approved by the Food and Drug Administration (FDA) for use in humans. Fluorescence occurs when incident light causes a fluorescent molecule to excite. It remains excited for a period of time (the fluorescence lifetime, $\square$ ) and then relaxes to the ground state by emitting a photon of lower energy (higher wavelength) than the energy of the incident light. The principle of fluorescence is illustrated using a Jablonski diagram in Figure 2.2. The fraction of excited fluorescent molecules that relax radiatively is known as the quantum efficiency.

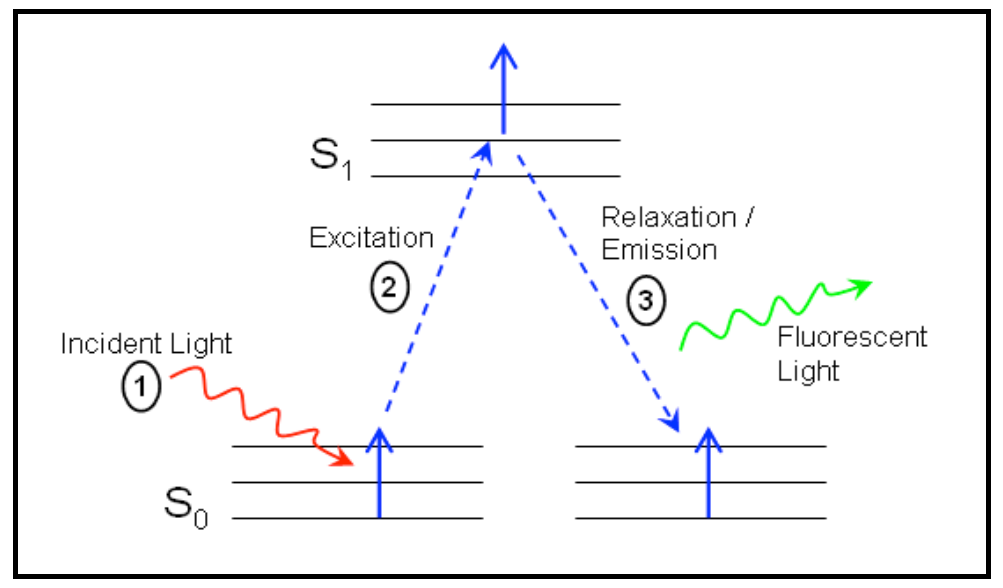

Figure 2.2 Jablonski diagram showing the principle of fluorescence.

The principle of fluorescence enhanced optical imaging is illustrated in Figure 2.3 for continuous-wave based imaging. When the fluorescent dye is injected into the tissue, it tends to accumulate close to the tumor site depending on its specificity. NIR light excited at the tissue surface propagates into the tissue and becomes attenuated due to absorption of 
the light by the tissue chromophores. Upon encountering a fluorescent molecule (or fluorophore), the molecule becomes excited (Figure 2.2). As the molecule relaxes back to the ground state, the energy is emitted at a higher wavelength (the fluorescent signal). The fluorescent signal propagates through the tissue becoming further attenuated by absorption. The emitted fluorescent light from the fluorophore combined with the original attenuated excitation light from the incident source is collected at the tissue surface using NIR sensitive detectors and appropriate filters to select the weak fluorescent signal.

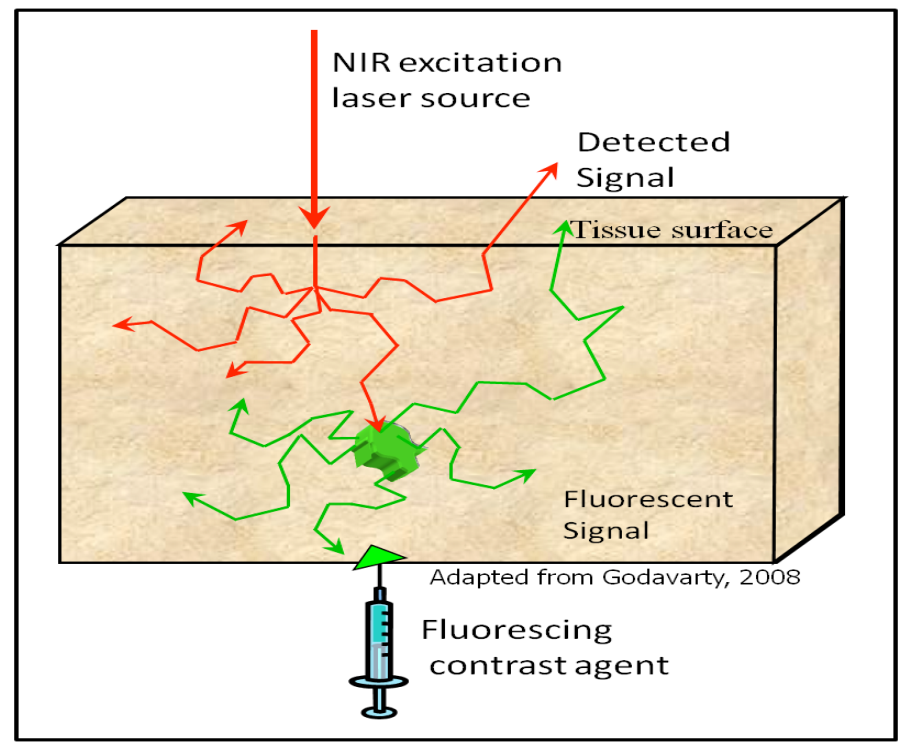

Figure 2.3 Principle of fluorescence enhanced optical imaging using continuous-wave based measurements.

\subsection{MEASUREMENT TECHNIQUES IN OPTICAL IMAGING}

Different measurements techniques are used in optical imaging which are timeindependent or time-dependent. The three types of measurement techniques used in optical imaging are continuous wave (which is time-independent), frequency-domain photon migration, and time-domain photon migration (which are time-dependent). Figure 2.4 
shows the basic principle of the three methods. The continuous wave $(\mathrm{CW})$ technique uses light intensity that is constant in time. The amplitude of the incident light attenuates as it passes through tissue and the amount that it attenuates is related to the optical properties of that tissue (Figure 2.4A). CW imaging is relatively simple, fast and inexpensive; however, one major drawback is that the absorption $\left(\mu_{\mathrm{a}}\right)$ and scattering $\left(\mu_{\mathrm{s}}\right)$ properties cannot be determined independently since information is collected as a single combined measurement. Frequency-domain photon migration (FDPM) uses intensity-modulated light waves that experience amplitude modulation decay and phase shift with respect to the incident wave (Figure 2.4B). The amplitude decay measures the absorption properties and the phase shift measures the scattering properties enabling each property to be measured independently. Time-domain photon migration (TDPM) uses nano- or pico-second laser pulses of incident light that broaden and attenuate according to the optical properties as they pass through the tissue (Figure 2.4C). The photon times-of-flight are recorded to acquire a photon distribution as a function of time. TDPM has good temporal resolution and is capable of measuring absorption and scattering properties independently. However, the instrumentation is more expensive and complex because it requires detectors with picoseconds or better resolution, and the method requires longer imaging time due to low signal-to-noise ratio (SNR) than CW or FDPM. 

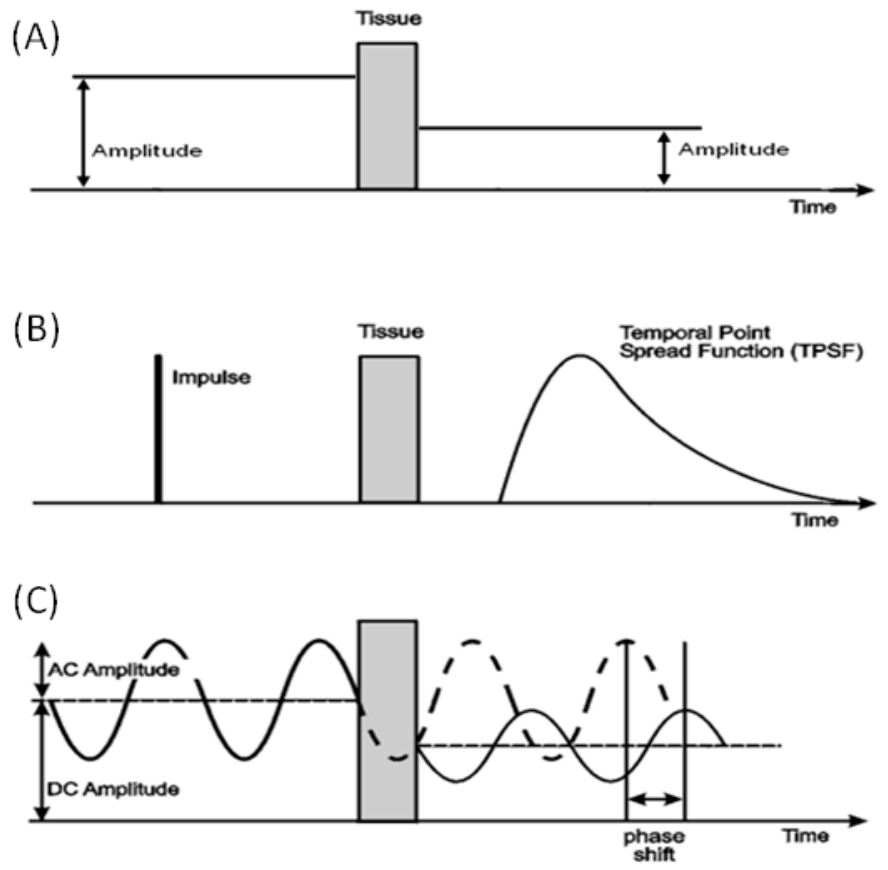

Figure 2.4 Diagram of the three measurement techniques for optical imaging: (A) continuous wave, (B) time domain photon migration, and (C) frequency domain photon migration (adapted from http://www.medphys.ucl.ac.uk/)

\subsection{MEASURMENT CONFIGURATIONS IN OPTICAL IMAGING}

Imaging systems can be designed for reflection and/or transillumination measurements. For reflection measurements, light that has passed through the tissue is diffusely reflected from the same side upon which the incident light was launched. For transillumination measurements, light that has passed though the tissue is diffusely transmitted from the side of the tissue opposite from which it was launched.

Different measurement configurations are used to perform optical imaging which determine the type of signal that will be collected from the tissue (reflectance and/or transillumination). The measurement geometries used in breast imaging are typically governed by the instrumentation, i.e. bulky instrumentation or hand-held device based instrumentation. Additionally, different combinations of point and area illumination are 
used. These different imaging geometries are described in detail in the following subsections.

Most three-dimensional (3D) optical imaging studies towards breast cancer diagnosis employ either compressed tissue based imaging configuration [7-12] (Fig 2.5A) or circularbased imaging configuration [13-28] (Fig 2.5B). The compressed tissue based configuration is analogous to x-ray mammography, and is disadvantageous due to patient discomfort from tissue compression and limited information obtained around the complete breast tissue. The circular-based configuration has minimal patient discomfort, but is limited by the bulky and non-portable instrumentation. The sub-surface based imaging configuration (Fig 2.5C) is a relatively new method that requires no tissue compression, and can be designed to mimic a portable and flexible imaging probe [27, 29-71]. In recent years, hand-held based optical imaging devices employing the sub-surface imaging configuration are developed in an attempt to translate the technology to the clinic, with maximum patient comfort and portability (compared to the available bulky optical imagers). A literature review of handheld based optical imaging devices developed to date is given in Chapter 3.

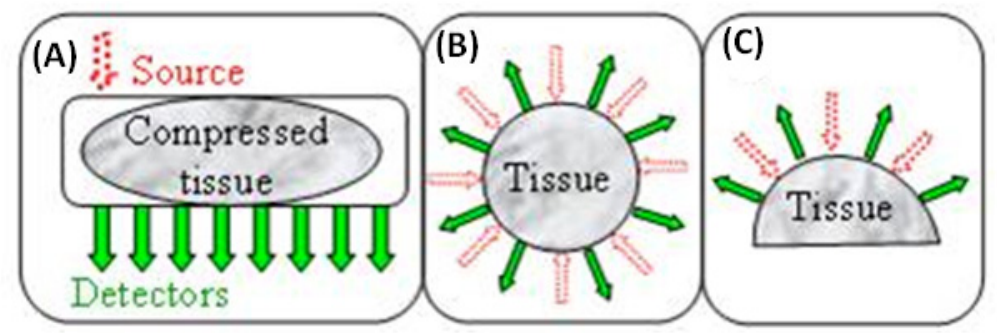

Figure 2.5 Different imaging configurations: (A) compressed tissue, (B) circular, and (C) subsurface Adapted from B. Jayachandran, 2007.[27] 


\subsection{IMAGING GEOMETRIES}

\subsubsection{Point source vs. area illumination and collection}

Different imaging geometries include the use of point source versus area illumination and collection. In area illumination and collection, the incident laser light from a single source is expanded to cover the entire tissue region being imaged at one time. The light is collected from the tissue over the same region by typically using a CCD camera based detector. This method has the advantages of rapid data acquisition and large volume interrogation, but requires bulky instrumentation and cannot be made to conform to the geometry of the tissue surface. Point source and detection based imaging uses optical fibers to illuminate and collect the light from the tissue surface. This method has the advantage of placing the fibers in direct contact with the tissue surface and can be made into a portable hand-held device based imaging system.

\subsubsection{Simultaneous illumination using point sources to approximate area illumination}

Point source and detector based imaging systems typically illuminate the source points sequentially, via optical fibers. An alternative approach involves simultaneous illumination of multiple source points. Simultaneous illumination enables rapid data acquisition and better approximates a more ideal case of area illumination since a large volume of tissue is interrogated in a single measurement. The hand-held device described herein employs simultaneous illumination of six source points in order to facilitate rapid data acquisition and larger volume interrogation as in area illumination. More details will be given in Chapter 6 . 


\subsection{INSTRUMENTATION}

Instrumentation for optical imaging is chosen based on the application. The basic components of any optical imaging system are a light source and a detector. A single source and detector pair are used for measuring single-point locations in the tissue which is termed optical spectroscopy. Multiple sources and detectors are required to collect measurements over an area of tissue which is termed optical imaging. The process of collecting measurements over an area and reconstructing the spatial optical property maps in $2 \mathrm{D}$ or $3 \mathrm{D}$ is termed optical tomography.

\subsubsection{Bulky instrumentation vs. hand-held devices}

Different research groups typically develop or use optical imaging instrumentation either in the form of an optical bed using the circular or compressed-tissue imaging configuration, or a hand-held probe using the subsurface based imaging configuration. Several research groups have developed optical imaging methods for breast imaging which use a bed upon which the subject lies prone while the breast is either suspended in a ring or cup, or compressed between two plates. An example of this type of instrumentation is shown in Figure 2.6A. This type of instrumentation allows for transillumination based imaging and control of the tissue geometry for ease in 3D image reconstruction. However, the instrumentation is bulky and non-portable and can be uncomfortable for the subject. Additionally, many systems use a matching fluid to compensate for lack of direct contact with the tissue. In order to overcome these limitations, several research groups have developed hand-held based optical imaging devices. Examples of these hand-held devices are shown in Figure $2.6 \mathrm{~B}$ and a complete literature review of the hand-held devices 
developed to date is given in Chapter 3. Hand-held devices have the advantages of being portable and patient-comfortable, and achieve full contact with various-shaped tissues. The devices developed to date are typically used to collect spectroscopic measurements of tissue optical properties and/or to estimate the 2D location of a tumor. The devices use point illumination/detection and reflectance based imaging geometry. None of the hand-held optical devices developed to date have attempted 3D tomography (without the addition of a second imaging modality) since they are unable to coregister the image to the tissue geometry. The device described herein is unique in its ability to perform 3D tomographic imaging using a hand-held NIR imaging device.

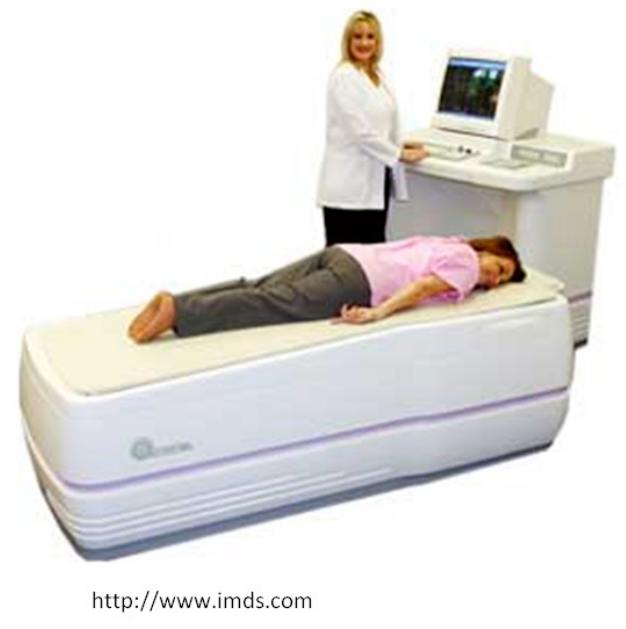

(A)
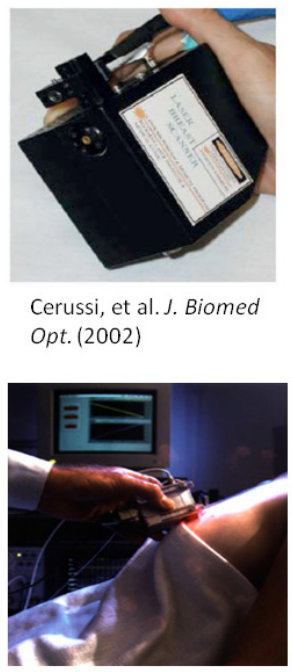

No KS, et al. IEEE Trans. Circuits (2005)

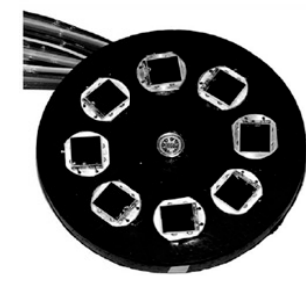

Chance B., et al. Acad. Radiol. (2005)

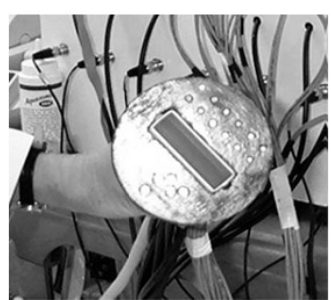

Zhu Q., et al.

(B)

Figure 2.6 Different types of instrumentation used for breast include: (A) bulky optical bed based imaging, and (B) hand-held device based imaging. Adapted from S. Erickson, 2009.[29] 


\subsubsection{Sources}

The most common types of sources used in diffuse optical imaging are laser diodes, light emitting diodes, halogen lamps, Ti:sapphire lasers, and helium-neon (He-Ne) long coherence lasers. An example of each type of source is shown in Figure 2.7. Laser diodes (Figure 2.7A) and light emitting diodes (Figure 2.7B) are semiconductors usually formed by a $\mathrm{p}-\mathrm{n}$ junction powered by an electrical current. Laser diodes of different NIR wavelengths are used according to the measured parameter of interest (e.g. oxyhemoglobin, deoxyhemoglobin, water, lipids, etc.). A halogen lamp (Figure 2.7C) uses a tungsten filament combined with halogen gas to produce white light of all wavelengths. Different filters can be used to select particular wavelengths appropriate to the imaging application. Ti:sapphire lasers (Figure 2.7D) are tunable lasers which can emit light in the NIR range between 650$1100 \mathrm{~nm}$. A He-Ne laser contains a mixture of helium and neon gas in a small bore capillary tube and is usually excited by a direct-current (DC) electrical discharge.

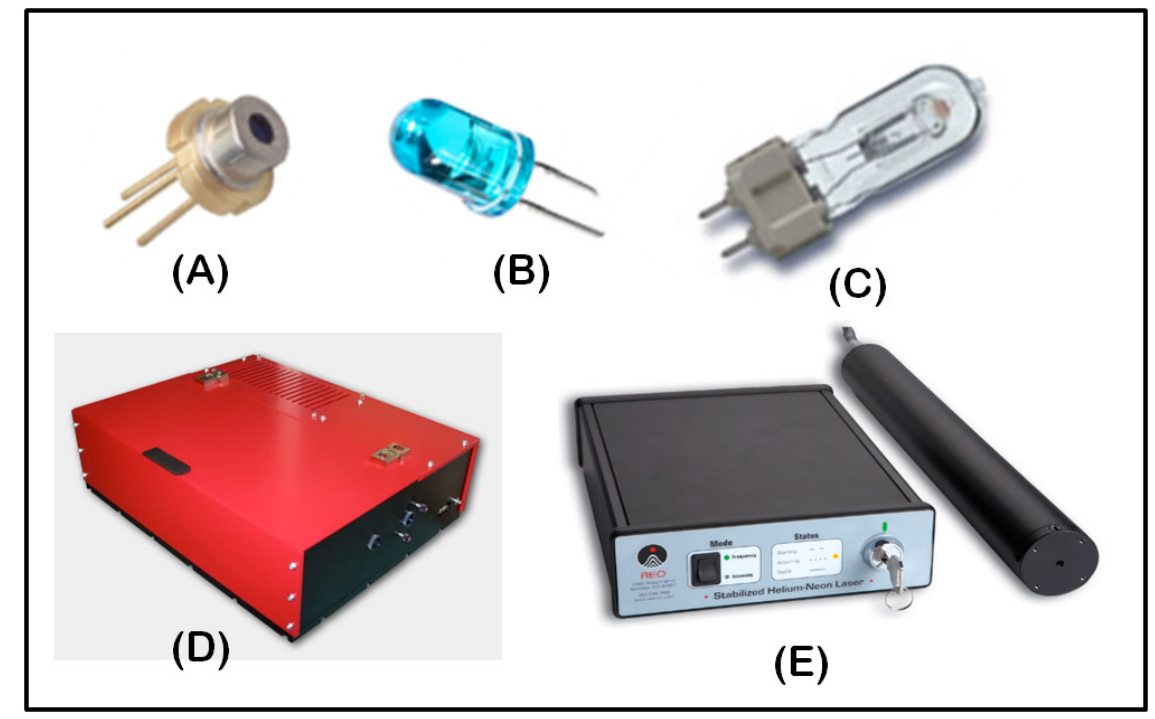

Figure 2.7 Types of sources commonly used in diffuse optical imaging: (A) laser diode http://fr.digikey.com/1/3/indexb31.html; (B) light emitting diode http://energyboomer.typepad.com; (C) 
halogen lamp http://shopzlot.biz/products/abco-halogen-lamp-50w-04423; (D) Ti:sapphire tunable laser http://www.dmphotonics.com/DPSS/DPSS_CWlasers_TiSa_pumping.htm; (E) helium-neon He-Ne long coherence laser http://www.newport.com

\subsubsection{Detectors}

The most common types of detectors used in diffuse optical imaging are charge-coupled device (CCD) cameras, avalanche photodiodes (APD), and photomultiplier tubes (PMT). An example of each type of detector is shown in Figure 2.8. A CCD camera (Figure 2.8A) converts optical light signal into electrical amplitude signals using multiple charge-coupled devices and reproduces the image of the subject. An avalanche photodiode (Figure 2.8B) is a highly sensitive semiconductor electronic photodetector device that converts light into electricity via the photoelectric effect. Photomultiplier tube detectors (Figure 2.8C) are designed to amplify the current of incident light up to 100 million times using multiple dynodes in order to detect very low intensity light signals.

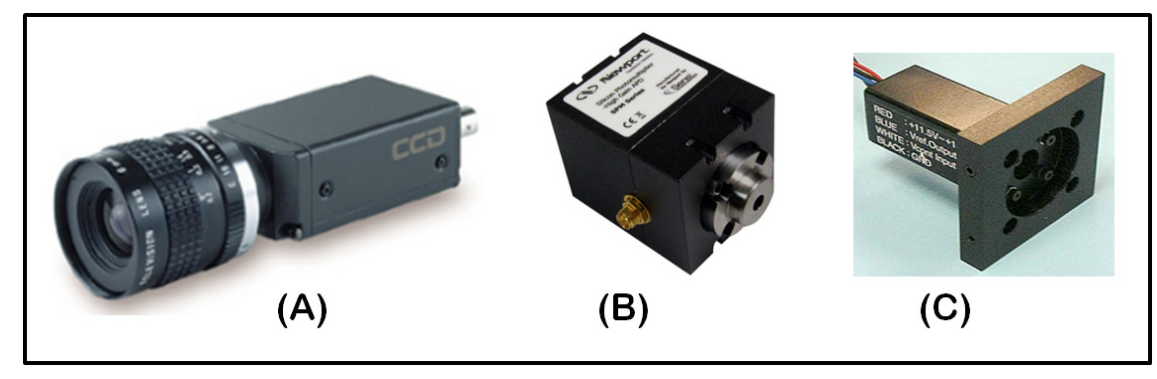

Figure 2.8 Types of detectors commonly used in diffuse optical imaging: (A) CCD camera http://www.aegis-elec.com/products/hitachi-KP-M3AN.html;

(B) avalanche photodiode http://www.newport.com; (C) photomultiplier tube http://www.prizmatix.com/docs/OptiBlocks.cfm.

Diffuse optical imaging is an emerging modality which noninvasively measures the optical properties (absorption and scattering) of near-infrared light in biological tissues. External contrast agents can be used to perform fluorescence-enhanced optical imaging toward detection of smaller tumors. Instrumentation is selected based on the different 
measurement techniques and imaging configurations for different applications of optical imaging in human tissues. The next three chapters give a review of past work performed by different research groups using optical imaging technology for breast tissue imaging. 


\section{CHAPTER 3}

\section{Past Work: Hand-held Optical Imaging Devices}

\subsection{INTRODUCTION}

Different hand-held based optical devices have been developed by several research groups [27,29-71] with differences in the instrumentation, capabilities, and their specific applications. The current review will focus on all the hand-held based devices (termed handheld imagers, probes, devices, and imaging systems by various researchers) developed to date towards optical imaging of biological tissues using NIR light. Table 3.1 provides a condensed summary of the different NIR hand-held devices developed to date in terms of the measurement technique, source and detector type, source wavelengths and input intensity, number of sources and detectors, target depth, and the number and type of subject studies (see Appendix I for extensive version of the table). Herein, the different hand-held devices are primarily discussed in terms of: (i) the measurement technique employed; (ii) imaging methods implemented; and (iii) their specific applications, along with the advantages and disadvantages of the different systems available to date.

\subsection{MEASUREMENT TECHNIQUES IN DIFFERENT HAND-HELD DEVICES}

Optical imaging is typically carried out using one of the three measurement techniques, namely, continuous wave (CW), frequency domain photon migration (FDPM), or time domain photon migration (TDPM). These measurement techniques are described in detail in section 2.4. Different research groups developing hand-held based optical devices employed different measurement techniques based on their specific application and focus of research. 
Table 3.1 Summary of different hand-held devices developed towards optical imaging (extensive table provided in Appendix I).

\begin{tabular}{|c|c|c|c|c|c|c|c|c|c|}
\hline $\begin{array}{c}\text { Device } \\
\# \\
\end{array}$ & Reference & Modality & \begin{tabular}{c|} 
Meas. \\
Technique
\end{tabular} & Source Type & $\begin{array}{c}\text { Source } \\
\text { Yavelength }\end{array}$ & \begin{tabular}{|c|} 
Detector \\
Type \\
\end{tabular} & $\begin{array}{l}\text { Tissue } \\
\text { Depth } \\
\end{array}$ & $\begin{array}{l}\text { Subject } \\
\text { Studied }\end{array}$ & $\begin{array}{l}\text { sources. } \\
\text { detectors }\end{array}$ \\
\hline \multirow{2}{*}{$1 a$} & $\begin{array}{c}\text { Tromberg. } 1997 \\
\text { [33] } \\
\end{array}$ & NIR-S & $\begin{array}{c}\text { FDPM } \\
(300 \mathrm{kHz}-1 \\
\text { GHz })\end{array}$ & $\begin{array}{c}\text { diode lasers } \\
(10-30=\mathbf{W})\end{array}$ & $\begin{array}{c}674,811,849 \\
956 \mathrm{~nm}\end{array}$ & $A P D$ & $1.0 \mathrm{~cm}$ & $\begin{array}{l}\text { human } \\
\text { subject }\end{array}$ & 1,1 \\
\hline & $\begin{array}{c}\text { Shah, } 2004 \\
{[39]}\end{array}$ & NIR-S & $\begin{array}{c}\text { FDPM } \\
(50-700 \mathrm{MHz})\end{array}$ & $\begin{array}{l}\text { diode lazer } z \\
(5-25=-1)\end{array}$ & $\begin{array}{c}674,780,803 \\
849,894,915 \\
980 \mathrm{~nm}\end{array}$ & $A P D$ & & $\begin{array}{l}\text { human } \\
\text { subject }\end{array}$ & 1,1 \\
\hline $1 b$ & $\begin{array}{c}\text { Cerussi, } 2007 \\
{[46]}\end{array}$ & NIR-S & $\begin{array}{c}C W \& \text { FDPM } \\
(50-1000 \mathrm{MHz})\end{array}$ & 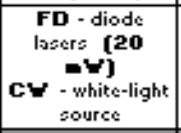 & $\begin{array}{c}660,690,780 \\
808,830,850 \\
\mathrm{~nm}\end{array}$ & $\begin{array}{c}\text { FD - APD } \\
\text { CW - CDD } \\
\text { detector }\end{array}$ & & $\begin{array}{l}\text { human } \\
\text { subject }\end{array}$ & $\begin{array}{c}2,2 \\
\text { (esquential } \\
\text { illumination) }\end{array}$ \\
\hline 2 & $\begin{array}{c}\text { No, } 2007 \\
{[49]}\end{array}$ & NIR-S & $\begin{array}{c}\text { FDPM } \\
(50-300 \mathrm{MHz})\end{array}$ & $\begin{array}{c}4 \text { laser diodes } \\
{[20=4]}\end{array}$ & $\begin{array}{c}681,783,823 \\
850 \mathrm{~nm}\end{array}$ & $A P D$ & & & $\begin{array}{l}4,1 \text { (ecquential } \\
\text { illuminstion) }\end{array}$ \\
\hline \multirow{2}{*}{3} & $\begin{array}{c}\text { Chance, } 2005 \\
{[50]}\end{array}$ & NIR-S & $\mathrm{CW}$ & $\begin{array}{c}\text { light emitting } \\
\text { diodes } \\
\text { [10-15 }=\text { A] }\end{array}$ & $\begin{array}{c}760,805,850 \\
\mathrm{~nm}\end{array}$ & $\begin{array}{l}\text { silicon } \\
\text { diode } \\
\text { detector? }\end{array}$ & $4.0 \mathrm{~cm}$ & $\begin{array}{l}\text { human } \\
\text { subject }\end{array}$ & 1,8 \\
\hline & $\begin{array}{c}\text { Nioka, } 2005 \\
\text { [51] }\end{array}$ & NIR-S & $\mathrm{CW}$ & $\begin{array}{l}\text { light emitting } \\
\text { diodes } \\
\text { [10-15 }=\text { A] }\end{array}$ & $\begin{array}{c}735,805,850 \\
\mathrm{~nm}\end{array}$ & $\begin{array}{l}8 \text { silicon } \\
\text { diode } \\
\text { detector? }\end{array}$ & $4.0 \mathrm{~cm}$ & $\begin{array}{l}\text { human } \\
\text { subject }\end{array}$ & 1,8 \\
\hline 4 & $\begin{array}{c}\text { Chance, } 2006 \\
{[53]}\end{array}$ & $\mathrm{NIR}-\mathrm{S}$ & $\begin{array}{l}\text { FDPM } \\
\text { (3kHz) }\end{array}$ & $\begin{array}{c}2 \text { light emitting } \\
\text { diodes } \\
\text { [20 =A] }\end{array}$ & $800 \mathrm{~nm}$ & $\begin{array}{c}\text { silicon diode } \\
\text { detector }\end{array}$ & $5.0 \mathrm{~cm}$ & $\begin{array}{l}\text { phantom \& } \\
\text { human }\end{array}$ & $\begin{array}{c}2,1 \\
\text { [simultancous } \\
\text { illuminstion) }\end{array}$ \\
\hline \multirow{2}{*}{7} & $\begin{array}{c}\text { Choe, } 2005 \\
{[58]}\end{array}$ & NIR-S & $\mathrm{CW}$ & $\begin{array}{l}\text { long coherence } \\
\text { Isoer }\end{array}$ & $785 \mathrm{~nm}$ & $A P D$ & & $\begin{array}{l}\text { human } \\
\text { subject }\end{array}$ & 4,4 \\
\hline & \begin{tabular}{|c|} 
Durduran, 2005 \\
[59] \\
\end{tabular} & NIR-S & $\mathrm{CW}$ & $\begin{array}{l}\text { long coherence } \\
\text { lazer }\end{array}$ & $785 \mathrm{~nm}$ & $\mathrm{APD}$ & $1.0 \mathrm{~cm}$ & $\begin{array}{l}\text { human } \\
\text { subject }\end{array}$ & 4,4 \\
\hline 8 & $\begin{array}{c}\text { Liebert, } 2005 \\
{[60]}\end{array}$ & NIR-S & TD & $\begin{array}{l}2 \text { picozecond } \\
\text { diode lazerz }\end{array}$ & $803,807 \mathrm{~nm}$ & PMT $=$ & & $\begin{array}{l}\text { humian } \\
\text { subject }\end{array}$ & 2,4 \\
\hline $9 a, b$ & $\begin{array}{c}\text { Sunar, } 2006 \\
\text { [61] }\end{array}$ & DCS \& DRS & $\begin{array}{c}\text { DCs - CW } \\
\text { DRs - } \\
\text { FDPM } \\
\mathrm{MHz})\end{array}$ & $\begin{array}{l}\text { DCs - long } \\
\text { coherence lazer } \\
\text { DRs - lazer } \\
\text { diodez }\end{array}$ & $\begin{array}{c}\text { DCS - } 785 \\
\text { nm DRS - } \\
690,785,830 \\
\mathrm{~nm} \\
\end{array}$ & APD & $1.5 \mathrm{~cm}$ & $\begin{array}{l}\text { human } \\
\text { subject }\end{array}$ & $\begin{array}{l}\text { DCS }-2,4 \\
\text { DRS }-2,4\end{array}$ \\
\hline $10 a$ & $\begin{array}{c}\text { Zhu, } 1999 \\
\text { [62] }\end{array}$ & $\begin{array}{c}\text { NIR } \text { क } \\
\text { Ultrszound }\end{array}$ & $\begin{array}{c}\text { FDPM } \\
(200 \mathrm{MHz})\end{array}$ & Iser diodes & $776.834 \mathrm{~nm}$ & $4 \mathrm{APD}=$ & $2.6 \mathrm{~cm}$ & phontom & 12,4 \\
\hline \multirow{2}{*}{$10 \mathrm{~b}$} & $\begin{array}{c}\text { Guvo, } 2000 \\
\text { [63] }\end{array}$ & $\begin{array}{l}\text { NIR \& } \\
\text { UItrszound }\end{array}$ & $\begin{array}{l}\text { FDPM } \\
\text { (140 MHz) }\end{array}$ & lazer diodes & $780,830 \mathrm{~nm}$ & 8PMT: & & phontom & 12,8 \\
\hline & $\begin{array}{c}\text { Chen, } 2001 \\
\text { [65] }\end{array}$ & $\begin{array}{c}\text { NIR \& } \\
\text { Ultrszound }\end{array}$ & $\begin{array}{c}\text { FDPM } \\
\text { (140 MHz) }\end{array}$ & Iseer diodes & $760,830 \mathrm{~nm}$ & $8 \mathrm{PMT}=$ & $2.5 \mathrm{~cm}$ & phantom & 12,8 \\
\hline 11 & $\begin{array}{c}\text { Zhu, } 2003 \\
\text { [66] }\end{array}$ & $\begin{array}{c}\text { NIR \& } \\
\text { Ultrszound }\end{array}$ & $\begin{array}{c}\text { FDPM } \\
\text { (140 MHz) }\end{array}$ & laser diodes & $780,830 \mathrm{~nm}$ & $8 \mathrm{PMT}=$ & & $\begin{array}{l}\text { human } \\
\text { subject }\end{array}$ & 12,8 \\
\hline
\end{tabular}




\subsection{MEASUREMENT TECHNIQUES IN DIFFERENT HAND-HELD DEVICES}

Optical imaging is typically carried out using one of the three measurement techniques, namely, continuous wave $(\mathrm{CW})$, frequency domain photon migration (FDPM), or time domain photon migration (TDPM). These measurement techniques are described in detail in section 2.4. Different research groups developing hand-held based optical devices employed different measurement techniques based on their specific application and focus of research.

\subsubsection{Continuous Wave (CW) Based Devices}

The CW measurement technique (which measures the attenuated light intensity that remains constant with time) has been employed by several of the devices developed for breast imaging, although using different sources and detectors. A CW-based device developed at the University of Pennsylvania (Device \#3, Table 3.1) employed light emitting diode sources (at three wavelengths) and silicon diode detectors [50-51]. Parallely, a different CW-based device (Device \#7, Table 3.1) was developed by another research group at the University of Pennsylvania [60,71], employing a long coherence laser source (4 sources) and fast photoncounting avalanche photodiodes as detectors (four of them) coupled via detector fibers. In contrast, a hand-held device (termed as a tissue oximeter) developed at the Ohio State University (Device \#6, Table 3.1) contained embedded laser diode and photodiode modules for CW-based NIR imaging [57-58,71].

Typically CW-based techniques are preferred over the time-dependent FDPM or TDPM measurement techniques, since the instrumentation is simple, inexpensive, and can 
be made portable towards developing hand-held based imaging devices. However, the CW technique provides limited depth information in comparison to the FDPM and TDPM techniques. This is because the technique only measures the changes in intensity, reflected from the combined effect of absorption and scattering (and not individually) [72-75] . In addition, if the CW-based hand-held devices were applied towards fluorescence-enhanced optical imaging studies, it would limit the technique from differentiating the changes in fluorescence intensity arising from the fluorophore concentration or its decay kinetics (i.e. fluorescence lifetime and quantum efficiency).

\subsubsection{Frequency-Domain Photon Migration (FDPM) Based Devices}

Most of the hand-held devices developed towards breast imaging employ FDPM technique due to the advantage of measuring amplitude and phase shift of the modulated signal separately. The following hand-held devices employ the FDPM measurement technique and are described in chronological order of device numbers in Table 3.1.

A multi-wavelength FDPM-based hand-held device (Device \#1a, Table 3.1) was developed at the University of California, Irvine [33-39]. The device is capable of working between $300 \mathrm{kHz}$ to $1 \mathrm{GHz}$ frequencies. Modulated light source is generated using laser diodes and a network analyzer (generates radiofrequency signals) and avalanche photodiodes (APD) are used as detectors, since they are capable of detecting the collected signals at such high frequency. A second generation of the device (Device \#1b, Table 3.1) was developed, which combines CW and FDPM methods into a single CW/FD probe [40-46]. The CW component was added for broad continuous wavelength coverage resulting in better 
chromophore identification and improved concentration quantification than that achieved using FD alone.

More recently, researchers from the University of California, Irvine miniaturized an entire FD imaging instrumentation into a single hand-held device (Device \#2, Table 3.1) [47-49] . Modulated light source is generated using a signal generator (10 MHz to $1 \mathrm{GHz}$ ) and a laser diode. A homodyne and heterodyne based imaging version of the device were developed and tested on homogeneous phantoms. The homodyne device was simpler, but the accuracy was compromised by noise, while the heterodyne device was more complex but the filtering of noise enabled greater accuracy [47] . The homodyne based device used a single frequency at the source and detector end, thus contaminating the detected signal with noise from the ambient light and harmonic frequency. Hence, a heterodyne based version of the mini-imaging device was developed, wherein two different frequencies were employed at the source and detector end (here $+45 \mathrm{MHz}$ difference between frequencies) in order to generate a new lower-frequency detected signal. The heterodyne approach (along with the use of a sharp crystal filter) filtered the noise due to ambient light and harmonic frequency, thus improving the sensitivity of signal detection. This first heterodyne version of the device used one detector for amplitude measurements of the $45 \mathrm{MHz}$ heterodyne signal alone, and a second detector for the regular phase measurements. Another version of the above device was developed into a full-heterodyne laser breast scanner, which used heterodyne technique for both amplitude and phase measurements [49]. The amplitude and phase results from a breast phantom using full-heterodyne matched those predicted 
by diffusion theory better than the results from the homodyne and the first heterodyne version of the devices [47].

Two multi-modality based imaging devices (Devices \#10a, \#10b, \#11, Table 3.1) were developed at the University of Connecticut that combined FDPM-based optical imaging and ultrasound into a single hand-held device [66-71]. The optical part of each system used 12 pairs of dual wavelength laser diodes $(780 \mathrm{~nm}$ and $830 \mathrm{~nm})$ and 8 photomultiplier tube (PMT) detectors. The two devices differ in the sourcedetector configuration as described in Section 3.3.

A device was developed at the University of Pennsylvania (Device \#4, Table 3.1) that used two simultaneous light sources, in contrast to the various frequency-domain based imaging devices developed by all researchers to date using sequential point source illumination. The device was based on an ac circuit that acquired low frequency $(3 \mathrm{kHz})$ measurements at a single wavelength from a pair of anti-phased (i.e. out of phase by $180^{\circ}$ ) light emitting diodes, using a silicon diode detector [53]. The device collected only amplitude changes, which are independent of frequencies, unlike the phase measurements that are optimal at higher frequencies of 100-200 MHz. Hence, even a low frequency based device was sufficient to provide an optimal signal to noise ratio (SNR) for this device [53], apart from reducing the instrumentation costs in comparison to a high frequency based device. The simultaneous anti-phased source illumination caused destructive interference of the photon density waves (PDW), which in turn was used to determine the 2-D spatial target localization of an absorbing (or fluorescing) target in a 3-D phantom(s) [7680]. 
A hand-held device was developed at the Huazhong University of Science and Technology (Device \#5, Table 3.1), which also uses the same principle of simultaneous anti-phased source illumination. The device used two pairs of light emitting diodes (LEDs) that produced $3 \mathrm{kHz}$ signals to form a low frequency phased array 2D fluorescence system [54]. One pair of LEDs was in-phase and the other was out-of-phase to produce two null planes, which in turn indicated the $2 \mathrm{D}$ location of a fluorescent target. The device was designed to provide real time detection and 2D localization of small fluorescent objects within a highly scattering medium.

The advantages of time-dependent (FDPM and TDPM) over time-independent $(\mathrm{CW})$ measurement techniques were described in section 2.4. In addition, FDPM is often

preferable over TDPM due to improved signal-to-noise ratio (SNR). Steady-state FDPM measurements in terms of amplitude and phase are minimally corrupted by the ambient light, since the instrument detects only modulated signals. Thus the FDPM instrument automatically acts as a filter for ambient light rejection, which is not only an advantage for FDPM over CW or TDPM techniques, but favored for clinical application in a non lightproof environment. Since hand-held optical devices are designed toward clinical translation, they typically employ the FDPM technique due to this advantage.

\subsubsection{Time-Domain Photon Migration (TDPM) Based Devices}

Time domain photon migration (TDPM) is not a favorable measurement technique for hand-held optical devices due to its expensive and complex instrumentation (as described in section 2.4). To date, only one TDPM-based hand-held device (Device \#8, Table 3.1) has been developed at Physikalisch-Technische Bundesanstalt in Berlin, Germany [60] for 
bedside assessment of cerebral perfusion in stroke patients. The device measured diffuse reflectance of 803 and $807 \mathrm{~nm}$ light from two picosecond diode lasers with pulse width of $\sim 100$ ps and $60 \mathrm{MHz}$ repetition rate. Detecting fiber bundles were used to collect the light and deliver it to photomultiplier tubes. The TDPM technique was chosen in this application because the measurement of variance in distributions of time of flight of photons can be used to monitor changes of absorption in the cortex as opposed to the skin or the skull.

\subsection{IMAGING METHODS OF DIFFERENT HAND-HELD DEVICES}

The hand-held imaging devices employing any one of the three measurement techniques use appropriate source-detector configurations to allow spectroscopic, tomographic, or interference-based imaging studies. The source-detector configuration governs the type and quality of information obtained using the imaging device (as described in section 2.8). Spectroscopic and tomographic imaging were described in section 2.8. In addition, the principle of interference can be used toward tumor localization. This method requires at least two sources that simultaneously illuminate in-phase (constructive interference) or outof phase (destructive interference), and typically detected using a single detector. The handheld optical devices developed can thus be categorized in terms of the method of imaging as: (i) spectroscopic imaging-based, (ii) tomographic imaging-based, and (iii) interference imaging-based. Schematics of the different source-detector configurations employed by the hand-held devices using the different imaging methods are shown in Figures 3.1, 3.2, and 3.3 for spectroscopy-based, tomography-based, and interference-based devices, respectively. 


\subsubsection{Spectroscopic Imaging-based Devices}

Spectroscopic imaging-based devices utilize the simplest source-detector configuration of a single source and single detector, in order to obtain spectroscopic information. The spectroscopic imaging-devices developed by a research group at the University of California, Irvine used the single source-detector design for characterization of breast tissue [33-46]. The Device \#1a in Figure 3.1 developed by this research group has a single source-detector pair to measure photon density wave phase and amplitude from which physiological properties of normal and tumor tissue are extracted [33]. The second generation of the same device (Device \#1b, Figure 3.1) used two source-detector pairs, of which one pair obtained FD measurements and the second pair obtained CW measurements [40]. The mini-FDPM device developed by researchers at the University of California, Irvine (Device \#2, Figure 3.1) also employed a single source (laser diode) and single detector (APD) that were housed within the device in order to make direct contact with the tissue surface [44-49].

The use of multiple sources and detectors enables measurements from several locations and tissue depths and provides more information for the reconstruction of the tissue optical 


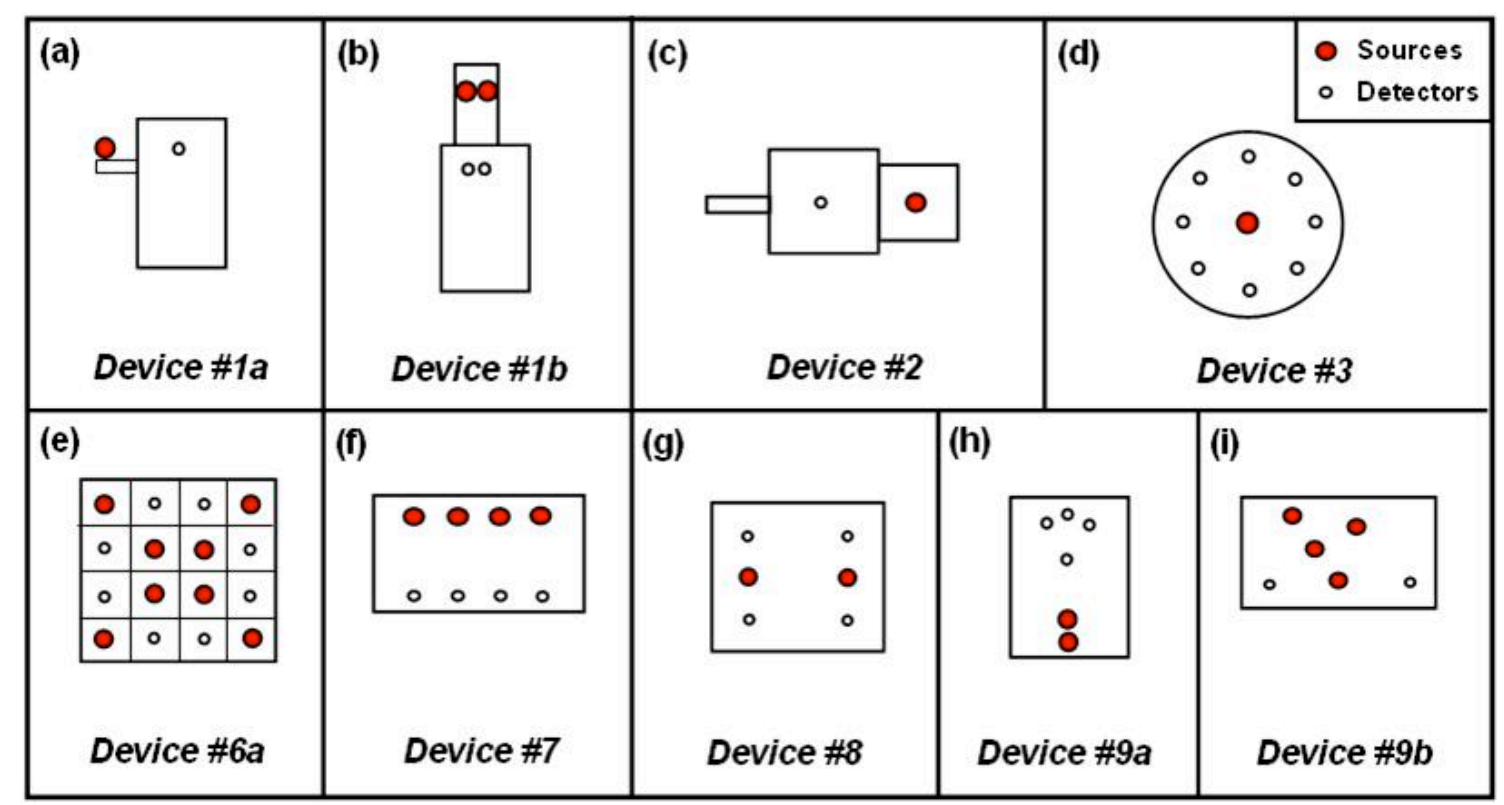

Figure 3.1 Schematics of spectroscopic imaging-based devices. (a) Device \#1a developed by researchers at the University of California, Irvine, which contains a single FD source-detector pair, (b) Device \#1b developed by the same group, which contains a single FD source-detector pair and a single CW source-detector pair, and (c) Device \#2 developed by other researchers at the same university, which contains a single source and detector embedded within the probe, (d) Device \#3 developed by researchers at the University of Pennsylvania, which contains a single source surrounded by 8 detectors, (e) Device \#6a developed by researchers at Ohio State University and ViOptix, Inc., which contains 8 sources and 8 detectors, (e) Device \#7 developed at the University of Pennsylvania, which contains 4 sources and 4 detectors, (f) Device \#8 developed at PhysikalischTechnische Bundesanstalt, which contains 2 sources and 4 detectors, (g) Device \#9a developed at the University of Pennsylvania, which contains 2 sources and 4 detectors, and (h) Device \#9b developed by the same group, which contains 4 sources and 2 detectors.

properties. Most of the spectroscopic imaging-based devices developed to date employ multiple sources and detectors in their probe design. The hand-held devices which used multiple sources and detectors are described below.

One of the research groups at the University of Pennsylvania [50], developed a device for breast imaging (Device \#3, Figure 3.1) consisting of a single LED source centered on the device that is surrounded by eight silicon diode detectors, each of which were located $4 \mathrm{~cm}$ from the source. This configuration enables the device to interrogate a larger tissue area than the use of a single detector would allow. Another research group in the same 
university [58-59] developed a device containing 4 sources lined up parallel to 4 detectors (Device \#7, Figure 3.1). The line of sources and line of detectors were separated by 2.5 $\mathrm{cm}$.

A breast imaging device developed at Ohio State University and ViOptix, Inc. (Device \#6a, Figure 3.1) contained 8 sources and 8 detectors arranged in a $2.5 \mathrm{~cm} \times 2.5 \mathrm{~cm}$ matrix [55-59] in order to image even larger areas. A second generation of the device was later developed (Device \#6b, Table 3.1) that contained 16 sources and 8 detectors to form a $3 \times 3 \mathrm{~cm}^{2}$ matrix [57]. In these devices the photodiode detectors collected the light simultaneously at a sampling rate of $100 \mathrm{kHz}$ while the 16 source fibers were illuminated sequentially.

- Several hand-held devices with multiple sources and detectors have also been developed towards brain imaging studies. The device developed at Physikalisch-Technische Bundesanstalt in Berlin, Germany (Device \#8, Figure 3.1) to measure cerebral perfusion in stroke patients contains 2 sources and 4 detectors [60]. One source and two detectors are positioned on each hemisphere of the head and four distributions of times of flight of photons were measured simultaneously at the 4 detector positions. Two other devices were developed at the University of Pennsylvania to monitor radiation therapy in patients with head and neck tumors [61]. One device contains 2 sources and 4 detectors with layout as shown in Figure 3.1 (Device \#9a) and the second device contains 4 sources and 2 detectors (Device \#9b, Figure 3.1). Both devices use NIR spectroscopic tools, but the former device is used to measure tumor blood flow before and during radiation therapy (method termed as diffuse correlation spectroscopy), and the latter 
device is used for quantification of the concentration of tissue chromophores in the head and neck regions (method termed as diffuse reflectance spectroscopy).

\subsubsection{Tomographic Imaging-based Devices}

The tomography imaging-based devices employ multiple sources and detectors in order to obtain 2D surface images as well as employ the measurements towards tomographic reconstructions. One group from the University of Connecticut developed hand-held NIR devices with multiple source-detector configurations combined with an ultrasound array [6271] as shown in Figure 3.2 (Devices \#10a, \#10b and \#11). The use of multiple sources and detectors enabled area imaging using the NIR component, and a priori information from the ultrasound component enabled 3D tomography, thus determining the target location.

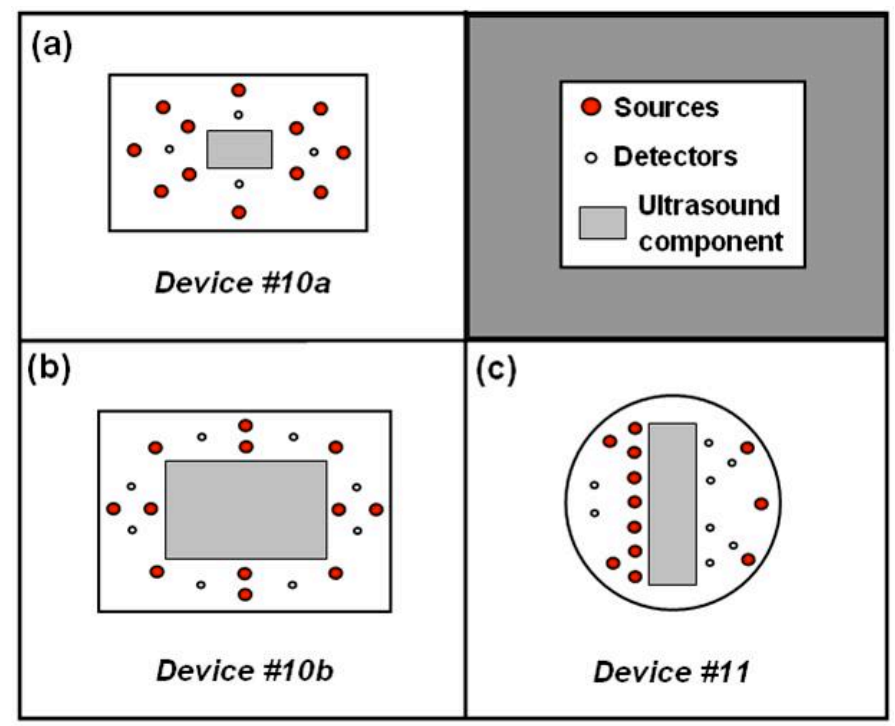

Figure 3.2 Schematics of tomographic imaging-based devices. (a) Device \#10a developed by researchers at the University of Pennsylvania and University of Connecticut, which contains a central ultrasound component surrounded by 12 sources and 4 detectors, (b) Device \#10b developed at the University of Connecticut, which contains an additional 4 detectors, and (c) Device \#11 developed by the same group, which contains an ultrasound component with 12 sources and 8 detectors. 


\subsubsection{Interference Imaging-based Devices}

All of the devices containing multiple sources are sequentially illuminated during imaging studies. In contrast, the interference imaging-based devices are designed and developed to perform simultaneous illumination of multiple sources for tumor location studies. The interference imaging-based device developed at the University of Pennsylvania [53] for breast

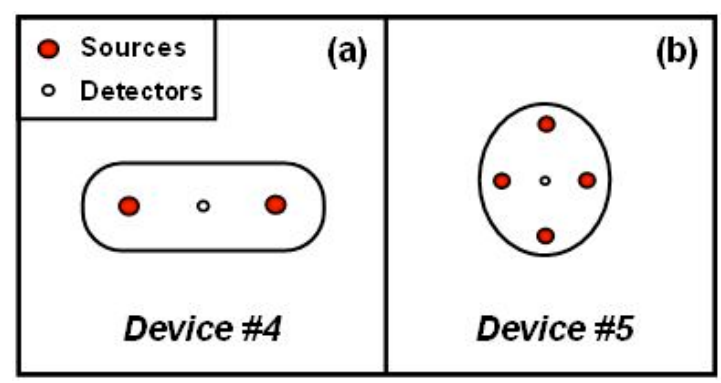

Figure 3.3 Schematics of interference imaging-based devices. (a) Device \#4 developed at the University of Pennsylvania, which contains a detector centered between two anti-phase sources for phase cancellation technique, (b) Device \#5 developed by researchers at the Huazhong University of Science and Technology, which contains a detector centered between a pair of in-phase sources and a pair of anti-phase which also uses the phase cancellation technique.

imaging employed two $800 \mathrm{~nm}$ LED sources that are illuminated simultaneously, but antiphase (i.e. out-of-phase) with respect to each other (Device \#4, Figure 3.3). A single silicon diode detector was placed mid-way (and in the same plane) between two sources, in order to detect and locate breast tumors based on the principles of destructive interference signals (as described in Section 3.3). A different breast imaging device developed at the Huazhong University of Science and Technology (Device \#5, Figure 3.3) employed two pairs of simultaneous light sources, wherein one pair was in-phase (to generate constructive interference pattern of the PDW) and the second pair was anti-phase (to generate destructive interference pattern of the PDW). The emitted optical signals were detected using a single 
fiber located at the center of the four sources in the probe that was connected to a photomultiplier tube (PMT) [54]. The in-phase and anti-phase sources interfere to create a null plane which was used to locate a target within the turbid medium. The target location was determined by the constructive or destructive interference pattern of the photon density waves (PDWs) in the presence of a tumor. This is a different approach towards real time localization of absorbing fluorescent objects within a highly scattering medium (in turn applicable for breast cancer localization).

\subsection{CLINICAL APPLICATIONS OF DIFFERENT HAND-HELD DEVICES}

The selection of measurement technique and source-detector layout is influenced by the application to which the hand-held optical imaging device will be used. Hand-held devices for NIR spectroscopy/imaging are used for several different applications related to the breast tissue (primarily), including measurement of normal and abnormal tissue physiological properties, tumor localization, and monitoring tumor changes during neoadjuvant chemotherapy treatment.

\subsubsection{Measurement of Normal and Abnormal Tissue Physiological Properties}

NIR spectroscopy has been used to show that the optical properties of normal breast tissue vary under different circumstances. Studies were conducted at the University of California, Irvine using the single source-detector device to demonstrate the differences in tissue physiological properties in pre- and post-menopausal subjects [35,40], as well as during different time periods of the menstrual cycle [38]. The results showed the ability of the NIR device to demonstrate wavelength-dependent differences in absorption and scattering properties between pre- and post-menopausal normal breast tissues as well as differences in the same breast tissue during different time periods in the menstrual cycle. It was also 
shown that the tissue properties vary both amongst different normal subjects, and also with respect to the different locations within the same breast of a normal subject [39].

One of the primary objectives of NIR spectroscopic imaging is to detect the presence of a tumor, based on the differences in the optical properties of normal versus diseased (tumor-bearing) tissue. Several hand-held devices have been developed toward detection of tumors within breast tissue:

Device \#1a in Table 3.1 was used to obtain measurements from normal and tumorcontaining human breast tissue [33]. Measurements were performed on two human subjects using a FDPM-based device, wherein one subject had fibroadenoma with ductal hyperplasia and the other had a benign fluid-filled cyst. The FDPM-based device was able to measure changes in absorption and scattering due to the presence of a small (1 cm diameter) lesion in the human breast. The data demonstrated differences in the concentrations of oxy- and deoxy-hemoglobin, and water in the normal versus tumor-bearing breast. A second detector was added to the above probe for a clinical study involving four human subjects with palpable lesions [34]. The second detector enabled two different source-detector distances (1.3 and 2.5 $\mathrm{cm})$ to be applied with each measurement, which provided information from different tissue depths. The results showed the differences in tissue optical properties between tumor and normal tissue, as well as between different types of lesions.

The combined continuous wave and frequency-domain (CW/FD) based device (Device \#1b, Table 3.1) developed at the University of California, Irvine was used to measure tissue properties in human subjects. The CW/FD based device was 
compared to a system that used only FD (Device \#1a, Table 3.1) in a study involving a total of 31 normal and abnormal human subjects [41]. The FD component used only a few discrete wavelengths within the range of $650-1000 \mathrm{~nm}$, whereas the CW component measured continuously across the entire range. The added bandwidth of imaged frequencies due to the $\mathrm{CW}$ measurements resulted in better chromophore identification and improved concentration quantification than using FD component alone. Another study was performed using the CW/FD based device in order to identify functional contrast in normal and abnormal breast tissue within a statistically relevant population of human subjects [45]. The spectra from tumor and normal tissue measured with the CW/FD based device were compared in 57 patients. The results showed differences in the optical properties of the tissue based on subject age, tumor size, and tumor pathology. Optical measurements obtained towards differentiating normal from diseased tissues were from lesions as small as $1 \mathrm{~cm}$, whose locations were determined a priori from standard x-ray mammography.

The full-heterodyne hand-held broadband breast scanning device developed at the University of California, Irvine (Device \#2, Table 3.1) was tested on breast and muscle phantoms [47] and an abnormal human subject [49]. The device was used to extract the optical properties (absorption and scattering coefficients) from phantom(s) and human tissue(s). Based on the measured differences in the absorption coefficients between the diseased (i.e. tumor) and normal breast tissue, the device detected the presence of a $6.36 \times 4.09 \mathrm{~cm}^{2}$ lesion in-vivo, located at a tissue depth of $0.5 \mathrm{~cm}$. The lesion size detected was relatively large compared to those detected by other hand-held devices, yet this device is relatively simple, compact, and 
an inexpensive design that is capable of extracting optical properties within a tissue volume [49].

The multi-detector hand-held device developed at the University of Pennsylvania (Device \#3, Table 3.1) was used in an extensive study to show the differences in the biochemical and physiological properties of breast tissue containing growing cancers, compared to corresponding normal tissue in the contralateral breast [50]. The use of multiple detectors increases the area and volume that can be interrogated with each scan, apart from increasing the overall data acquisition rates. Also, a source-detector separation of $4 \mathrm{~cm}$ has a significant probability of detecting a target at a depth of 4 cm or more [50]. In a study involving 116 subjects recruited over six years, CW measurements were obtained on both the normal and tumor-bearing breast tissues of human subjects. The smallest lesion detected was $0.8 \mathrm{~cm}$ and the greatest tissue depth reached was $4 \mathrm{~cm}$. The results demonstrated the ability of the device to detect the presence of lesions based on measured values of angiogenesis and hypermetabolism. The difference in these values between the normal and the tumor bearing breast indicated the presence of cancer. All the subjects involved in the above study were patients undergoing biopsy, and hence the lesion location was known a priori using a different imaging modality.

The hand-held device (termed as tissue oximeter by the researchers) designed by $\mathrm{Xu}$, et al (Device \#6, Table 3.1) was used for real time imaging of tissue oxygen saturation and hemoglobin concentrations [55-57]. Simulation experiments and sensitivity analysis were performed with respect to tumor size, tumor depth, tumor lateral location, and optical contrast [55]. The maximum measurement depth was $1.5 \mathrm{~cm}$ 
with absorption sensitivity of $0.1 \mathrm{~cm}$. The device was used in a study in conjunction with ultrasound to image an abnormal breast in a human subject [56]. The results from combining NIR spectroscopy and ultrasound modalities demonstrated 3D tomographic imaging (towards 3D target localization) of functional parameters of a breast lesion and the surrounding normal tissue. 3D reconstruction was performed after the added information of a second imaging modality (ultrasound). The actual lesion size imaged for this study was $0.58 \times 0.41 \times 0.51 \mathrm{~cm}^{3}$. In another study, the same device was used (without ultrasound) to test the effect of compression on human calf muscle and human breast tissue in vivo [57]. The homogeneous optical property in terms of absorption coefficients was determined for these tissues, using analytical solution of the diffusion equation in semi-infinite/infinite medium along with the $2 \mathrm{D}$ and $3 \mathrm{D}$ experimental images.

A hand-held device (Device \#7, Table 3.1) was designed to measure blood flow heterogeneity [58-59] in order to show that blood flow in a breast containing a palpable tumor is different than that in a normal breast. The device was used to obtain measurements from a $2.0 \mathrm{~cm}$ diameter lesion located at a depth of $1.0 \mathrm{~cm}$. The results showed that blood flow was greater in the tumor region than in the normal tissue.

\subsubsection{Tumor Localization and Characterization}

Several hand-held devices have been designed not only to detect the presence of a tumor, but also to determine the $2 \mathrm{D}$ location of the lesion within a breast using NIR optical imaging. 
The ac circuit based device developed at the University of Pennsylvania (Device \#4, Table 3.1) was used in phantom(s) and in-vivo human studies as well [53]. As described in Section 3.2, the device worked by phase cancellation from the two light sources. The device was scanned across the breast or phantom and when the presence of a target was detected, a change in sound or light on the device indicated the $2 \mathrm{D}$ location of the lesion. The device was able to detect the presence and $2 \mathrm{D}$ location of phantom targets as small as $1.0 \mathrm{~cm}^{3}$, which were located up to a depth of $5 \mathrm{~cm}$.

The only hand-held device that was used for fluorescence measurements has been developed at the Huazhong University of Science and Technology (Device \#5, Table 3.1). A study was performed with a phantom containing a $1.0 \mathrm{~cm}^{3}$ target with 0.8 $\mu \mathrm{M}$ ICG, located at a depth of $0.8 \mathrm{~cm}$ [54]. The device uses the phase cancellation technique (as described in Section 3.2) with a combination of constructive and destructive interference patterns in order to locate the fluorescent target.

These hand-held devices demonstrate the ability to indicate the $2 \mathrm{D}$ location of a target from surface measurements based on phase cancellation of PDWs. They have the advantage of being simple and compact and give $2 \mathrm{D}$ target locations even prior to any tomographic imaging.

One major goal of optical imaging is to not only localize a lesion, but also to differentiate if the lesion is benign or malignant. Conventional imaging techniques such as x-ray mammography and breast ultrasound have limited ability to differentiate benign and malignant lesions leading to large numbers of benign biopsies [67]. Optical imaging using a single wavelength can measure optical absorption, which is related to tumor vasculature. 
When multiple wavelengths are used, oxyhemoglobin and deoxyhemoglobin can be measured and give information regarding tumor malignancy [69].

Several versions of a multi-modal hand-held device (Devices \#10a, \#10b, \#11, Table 3.1) have been developed at the University of Connecticut [62-71] which combine NIR optical imaging and ultrasound into a single hand-held device. The optical information using multiple wavelengths can better determine the tumor malignancy $[67,69]$ and the ultrasound component provides a priori information which enables tumor localization by 3D reconstruction [65]. The device was used to image and differentiate benign and malignant lesions in human subjects. The lesions were successfully reconstructed using the a priori information from the ultrasound component and it was found that malignant lesions had a much higher maximum total hemoglobin concentration due to increased angiogenesis $[67,69]$.

\subsubsection{Monitoring Tumor Changes during Neoadjuvant Chemotherapy}

Another important application of optical hand-held devices is to monitor the progression of a tumor over time as chemotherapy treatment is administered. Neoadjuvant chemotherapy treatment is administered to a cancer patient prior to surgery in an attempt to decrease the size of the tumor. Several studies have been conducted toward this application.

The CW/FD based device developed at the University of California, Irvine (Device \#1b, Table 3.1) was used in several studies conducted in order to monitor tumor response to neoadjuvant chemotherapy in patients $[42-43,46]$. The tumor locations were known a priori from mammography, ultrasound, or palpation. The device was used to measure the physiological characteristics of the tumor prior to chemotherapy. This was followed by monitoring the changes in tissue optical 
properties over several months, due to changes in the tumor with neoadjuvant chemotherapy treatment.

In a different study, NIR spectroscopic imaging using the same CW/FD device was performed along with MRI in order to monitor the response to neoadjuvant chemotherapy in a human subject [43]. The initial lesion size was $3.8 \times 4.3 \times 4.3 \mathrm{~cm}^{3}$ before treatment and $3.0 \times 2.4 \times 2.3 \mathrm{~cm}^{3}$ after treatment. The NIR spectroscopy measurements provided functional information in terms of absolute hemoglobin concentrations and tumor hemoglobin oxygen saturation, while the MRI provided anatomical information. The final images were a combination of the functional information from NIR spectroscopy and the structural information from MRI.

Researchers from the University of Connecticut used their multi-modal hand-held device (Device \#11, Table 3.1) to image heterogeneous hemoglobin distributions in large cancers, which are used to monitor tumor vascular responses to neoadjuvant chemotherapy [70]. Tumors ranging from 2.5 to $4 \mathrm{~cm}$ were monitored in six patients. The studies showed that after several cycles of neoadjuvant chemotherapy the measured values of total hemoglobin concentration and spatial extension were much smaller in comparison to those prior to chemotherapy.

Most of the applications using the different hand-held devices were involved with the breast tissue and hence described in detail above. In addition, there has been applications of handheld based NIR devices towards cerebral perfusion in stroke patients (Device \#8, Figure 3.1), and monitoring radiation therapy in patients with head and neck tumors (Devices \#9a, 9b, Figure 3.1). 


\subsection{DISCUSSION AND CONCLUSION}

Many hand-held based NIR devices have been developed for optical spectroscopy and imaging applications. Apart from being non-invasive and non-ionizing, the advantages of hand-held optical imaging devices are that they are also portable, relatively inexpensive, and patient-comfortable because they do not require tissue compression. However, optical measurements can be easily contaminated by motion artifacts caused by patient or operator as well as relative motion of the probe and soft tissue during contact imaging, similar to $1 \mathrm{D}$ ultrasound probes scanned in 2D to perform 3D imaging. Careful operation procedure and operator training are required to obtain good quality images using hand-held based optical devices over using bulky optical systems employing compression plates or circular geometry.

The optimal design of a hand-held device is determined by the parameter(s) to be measured and the specific applications it is implemented towards. The hand-held devices developed to date primarily use CW and/or FDPM measurement techniques. FDPM has the added advantage over $\mathrm{CW}$ of collecting both amplitude and phase information separately and thus provides improved optical contrast and greater depth information [77]. The handheld devices also differ in the source-detector configuration, which is primarily governed by the desired applications they are developed towards. A single source and detector design has been used for spectroscopic measurements of physiological properties. Dual sources illuminated simultaneously and out-of-phase have been used for target location by detection of phase cancellation. Multiple sources and detectors have been configured towards imaging a given area of the tissue.

The overall goal of any hand-held based optical device is to accelerate the clinical translation of the technology, with maximum patient comfort and portability (compared to 
the many bulky optical imagers available). However, all of the hand-held optical devices available to date have only flat probe heads, which limit them from contouring to tissue curvatures with good surface contact. The flat probe head design also limits the devices to collecting only diffuse reflectance measurements, and not transilluminated measurements. Independent of the measurement technique (CW, FDPM, or TDPM) employed, reflectancebased measurements provide limited depth information and depth recovery of tumors in comparison to transillumination-based measurements [3] (wherein the light is allowed to propagate through tissue depths and NIR signals are collected on opposite surfaces with respect to the illuminating surface).

In addition, most of the applications of these hand-held based optical devices are limited to spectroscopic measurements of physiological properties and 2D target localization (without tomographic analysis). To date, these hand-held optical devices (or imagers) have not been successful in demonstrating 3D tomographic imaging of tissue phantoms or real tissues using NIR imaging alone (with or without fluorescence), since the source and detector points are not co-registered onto the tissue contours. In order to successfully reconstruct a lesion size and location in three dimensions, a priori information from another imaging modality (e.g. ultrasound) was required [65-70].

The hand-held optical imager developed in our laboratory is unique from other devices in that it is capable of performing 3D tomography (using NIR imaging alone) via implementation of self-coregistration facilities. The features and instrumentation of the device are described in detail in Chapter 6. 


\section{CHAPTER 4}

\section{Past Work: Fluorescence Tomography Experimental Studies}

\subsection{INTRODUCTION}

Fluorescence-enhanced diffuse optical tomography (FDOT) has been explored by many research groups in the past three decades.[3,81-133] This chapter gives a review of the recent literature from 2008 to the present. A literature review of image reconstruction using experimental data from fluorescence tomography (FT) studies up to the year 2007 is provided in Appendix B. Table 4.1 provides a summary of the experimental research performed in fluorescence tomography from 2008 to the present. The work performed by different research groups is summarized here in terms of (i) efforts to improve the reconstruction algorithm for fluorescence tomography, (ii) efforts to develop and/or improve instrumentation for fluorescence tomography, and (iii) efforts to improve experimental methods of fluorescence tomography.

\subsection{RECONSTRUCTION ALGORITHM FOR FLUORESCENCE TOMOGRAPHY}

Reconstruction of fluorescent optical data is mathematically challenging due to the illposed $^{18}$ nature of the inversion problem. Several research groups are currently working to improve the computational aspect of fluorescence tomography.

\footnotetext{
${ }^{18}$ Having more than one solution.
} 
Table 4.1 Summary of the experimental research performed in fluorescence tomography from 2008 to the present

\begin{tabular}{|c|c|c|c|c|c|c|c|c|c|c|}
\hline \multirow[b]{2}{*}{ Reference } & \multicolumn{4}{|c|}{ Phantom / Animal } & \multirow{2}{*}{$\begin{array}{l}\text { Measure } \\
\text { ment } \\
\text { Method }\end{array}$} & \multicolumn{2}{|c|}{ Instrumentation } & \multicolumn{3}{|l|}{ 2D } \\
\hline & $\begin{array}{l}\text { Backgroun } \\
\text { d }\end{array}$ & Target & $\begin{array}{l}\text { Contrast } \\
\text { Agent }\end{array}$ & Ratio & & $\begin{array}{l}\text { Source } \\
\text { (geometry) }\end{array}$ & $\begin{array}{l}\text { Detector } \\
\text { (geometry) }\end{array}$ & $\begin{array}{l}\text { or } \\
3 D\end{array}$ & $\begin{array}{l}\text { Forward } \\
\text { Method }\end{array}$ & $\begin{array}{l}\text { Inverse } \\
\text { Method }\end{array}$ \\
\hline $\begin{array}{l}\text { (Davis, et } \\
\text { al, 2008) } \\
{[121]}\end{array}$ & $\begin{array}{l}\text { DPBS, 1\% } \\
\text { intralipid, } \\
\text { ICG, \& } \\
\text { India ink }\end{array}$ & $\begin{array}{l}\text { Plastic } \\
\text { cylinder } \\
\mathrm{R}=1 \mathrm{~cm}\end{array}$ & ICG & 3.3:1 & $\begin{array}{l}\text { CW (w/ } \\
\text { MRI) }\end{array}$ & $\begin{array}{l}690 \mathrm{~nm} \text { laser } \\
\text { diode (point) }\end{array}$ & CCD (point) & $3 \mathrm{D}$ & $\begin{array}{l}\text { Diff. } \\
\text { Approx. }\end{array}$ & $\begin{array}{l}\text { Laplacian } \\
\& \\
\text { Jacobian }\end{array}$ \\
\hline $\begin{array}{l}\text { (Kepshire, } \\
\text { et al, 2008) } \\
{[115]}\end{array}$ & $\begin{array}{l}\text { Water, } 2 \% \\
\text { India ink, } \\
5 \% \text { Tween- } \\
20, \quad 1 \% \\
\text { intralipid }\end{array}$ & $\begin{array}{l}\text { Cylinder } \\
\mathrm{R}=4 \mathrm{~mm}\end{array}$ & $P p-I X$ & $\begin{array}{l}3.5: 1 \quad- \\
10: 1\end{array}$ & CW & $\begin{array}{l}635 \mathrm{~nm} \text { laser } \\
\text { diode (area) }\end{array}$ & CCD (area) & $2 \mathrm{D}$ & $\begin{array}{l}\text { Diff. } \\
\text { Approx. }\end{array}$ & $\begin{array}{l}\text { FluoroFAS } \\
\mathrm{T} \\
\text { (software } \\
\text { package) }\end{array}$ \\
\hline $\begin{array}{l}(\mathrm{Ge}, \text { et al, } \\
2008) \\
{[3]}\end{array}$ & $\begin{array}{l}1 \% \text { Liposyn } \\
(10 \times 10 \times 6.5 \\
\mathrm{cm})\end{array}$ & $\begin{array}{l}\text { Plastic } \\
\text { sphere } \\
\mathrm{R}=0.475 \\
\mathrm{~cm}\end{array}$ & ICG & $1: 0$ & FDPM & $\begin{array}{l}785 \mathrm{~nm} \text { laser } \\
\text { diode (point) }\end{array}$ & $\begin{array}{l}\text { ICCD } \\
\text { (point) }\end{array}$ & $3 D$ & FEM & AEKF \\
\hline $\begin{array}{l}\text { (Mohajerani } \\
\text { et al, } \\
2009) \\
{[122]}\end{array}$ & Nu/Nu mice & $\begin{array}{l}4 \mathrm{~T}-1 \\
\text { murine } \\
\text { breast } \\
\text { adenocarci } \\
\text { noma cells }\end{array}$ & $\begin{array}{l}\text { ProSen } \\
\text { se }\end{array}$ & in-vivo & CW & -- & -- & $3 \mathrm{D}$ & $\begin{array}{l}\text { Diff. } \\
\text { Approx. }\end{array}$ & $\begin{array}{l}\text { MSE \& } \\
\text { coincidenc } \\
\text { e mask }\end{array}$ \\
\hline $\begin{array}{l}\text { (Davis, et } \\
\text { al, 2009) } \\
{[123]}\end{array}$ & $\begin{array}{l}\text { Liquid } \\
\text { (water, 1\% } \\
\text { intralipid) } \\
\text { Solid } \\
\text { (gelatin, } \\
\mathrm{TiO}_{2} \text {, blood) }\end{array}$ & $\begin{array}{l}2 \text { small } \\
\text { heterogenei } \\
\text { ties }\end{array}$ & LuTex & $\begin{array}{l}2.5: 1 \\
3: 1\end{array}$ & CW & $\begin{array}{l}690 \mathrm{~nm} \text { laser } \\
\text { diode (point) }\end{array}$ & CCD (point) & $2 \mathrm{D}$ & FEM & $\begin{array}{l}\text { diffusion- } \\
\text { based } \\
\text { optimizatio } \\
n\end{array}$ \\
\hline $\begin{array}{l}\text { (Ziegler, et } \\
\text { al, 2009) } \\
{[124]}\end{array}$ & $\begin{array}{l}\text { rectangular } \\
\text { cuvette } \\
(25 \times 25 \times 6 \\
\left.\mathrm{cm}^{3}\right)\end{array}$ & $\begin{array}{l}\text { delrin twin } \\
\text { cones }\end{array}$ & $\begin{array}{l}\text { Omocy } \\
\text { anine }\end{array}$ & $5: 1$ & TDPM & $\begin{array}{l}\text { Ti:sapphire } \\
\text { laser (point) }\end{array}$ & $\begin{array}{l}\text { GaAs-PMT } \\
\text { (point) }\end{array}$ & $3 \mathrm{D}$ & $\begin{array}{l}\text { Diff. } \\
\text { Approx. }\end{array}$ & $\begin{array}{l}\text { Rytov } \\
\text { approx. }\end{array}$ \\
\hline $\begin{array}{l}\text { (Deliolanis, } \\
\text { et al, 2009) } \\
{[125]}\end{array}$ & Nude mice & $\begin{array}{l}\text { Lewis lung } \\
\text { carcinoma } \\
\text { cells }\end{array}$ & $\begin{array}{l}\text { mCherr } \\
\text { y } \\
\text { fluores } \\
\text { cent } \\
\text { protein }\end{array}$ & in-vivo & CW & $\begin{array}{l}593 \text { nm, } 750 \\
\mathrm{~nm} \quad \text { laser } \\
\text { diode (area) }\end{array}$ & CCD (area) & $3 D$ & none & ART \\
\hline $\begin{array}{l}\text { (Kepshire, } \\
\text { et al, 2009) } \\
{[126]}\end{array}$ & $\begin{array}{l}\text { Phantom } \\
\text { from ART, } \\
\text { Inc. }\end{array}$ & $\begin{array}{l}\text { Sphere } \\
\mathrm{R}=4 \mathrm{~mm}\end{array}$ & $\mathrm{Pp}-\mathrm{IX}$ & $1: 0$ & $\begin{array}{l}\text { CW } \\
\text { (noncont } \\
\text { act) }\end{array}$ & $\begin{array}{l}635 \mathrm{~nm} \text { laser } \\
\text { diode (point) }\end{array}$ & $\begin{array}{l}4 \quad \text { PMTs } \\
\text { (point) }\end{array}$ & $2 D$ & none & NIRFAST \\
\hline $\begin{array}{l}(\mathrm{Ge}, \text { et al, } \\
2009) \\
{[127]}\end{array}$ & $\begin{array}{l}1 \% \text { Liposyn } \\
(10 \times 10 \times 6.5 \\
\mathrm{cm})\end{array}$ & $\begin{array}{l}\mathrm{R}=0.29- \\
0.475 \mathrm{~cm}\end{array}$ & ICG & $\begin{array}{l}5: 1 \\
1: 0\end{array}$ & FDPM & $\begin{array}{l}785 \mathrm{~nm} \text { laser } \\
\text { diode (point) }\end{array}$ & $\begin{array}{l}\text { ICCD } \\
\text { (point) }\end{array}$ & $3 \mathrm{D}$ & FEM & AEKF \\
\hline $\begin{array}{l}\text { (Lin, et al, } \\
2010) \\
{[128]}\end{array}$ & $\begin{array}{l}\text { Agarose } \\
\text { powder, } \\
\text { India ink, } \\
\text { intralipid } \\
\text { (25 mm } \\
\text { diameter) }\end{array}$ & $\begin{array}{l}\text { Glass tube } \\
\mathrm{R}=1.2 \mathrm{~mm}\end{array}$ & ICG & $\begin{array}{l}1: 1- \\
25: 1\end{array}$ & $\begin{array}{l}\text { CW (w/ } \\
\text { DOT \& } \\
\text { XCT) }\end{array}$ & $\begin{array}{l}785 \mathrm{~nm}, 830 \\
\mathrm{~nm} \text { laser } \\
\text { (point) }\end{array}$ & CCD (area) & $2 \mathrm{D}$ & $\begin{array}{l}\text { Coupled } \\
\text { Diff. Eq. }\end{array}$ & Laplacian \\
\hline $\begin{array}{l}\text { (Hyde, et al, } \\
2010 \text { ) } \\
{[129]}\end{array}$ & $\begin{array}{l}\text { Transgenic } \\
\text { mice }\end{array}$ & $\begin{array}{l}\text { Amyloid- } \\
\text { beta } \\
\text { plaques }\end{array}$ & $\begin{array}{l}\text { Oxazin } \\
\text { e } \\
\text { derivati } \\
\text { ve } \\
\text { probe }\end{array}$ & in-vivo & CW & $\begin{array}{l}650 \mathrm{~nm} \text { laser } \\
\text { diode (area) }\end{array}$ & CCD (area) & $3 D$ & $\begin{array}{l}\text { Diff. } \\
\text { Approx. }\end{array}$ & $\begin{array}{l}\text { Tikhonov } \\
\text { w/ } \\
\text { structural } \\
\text { priors }\end{array}$ \\
\hline
\end{tabular}




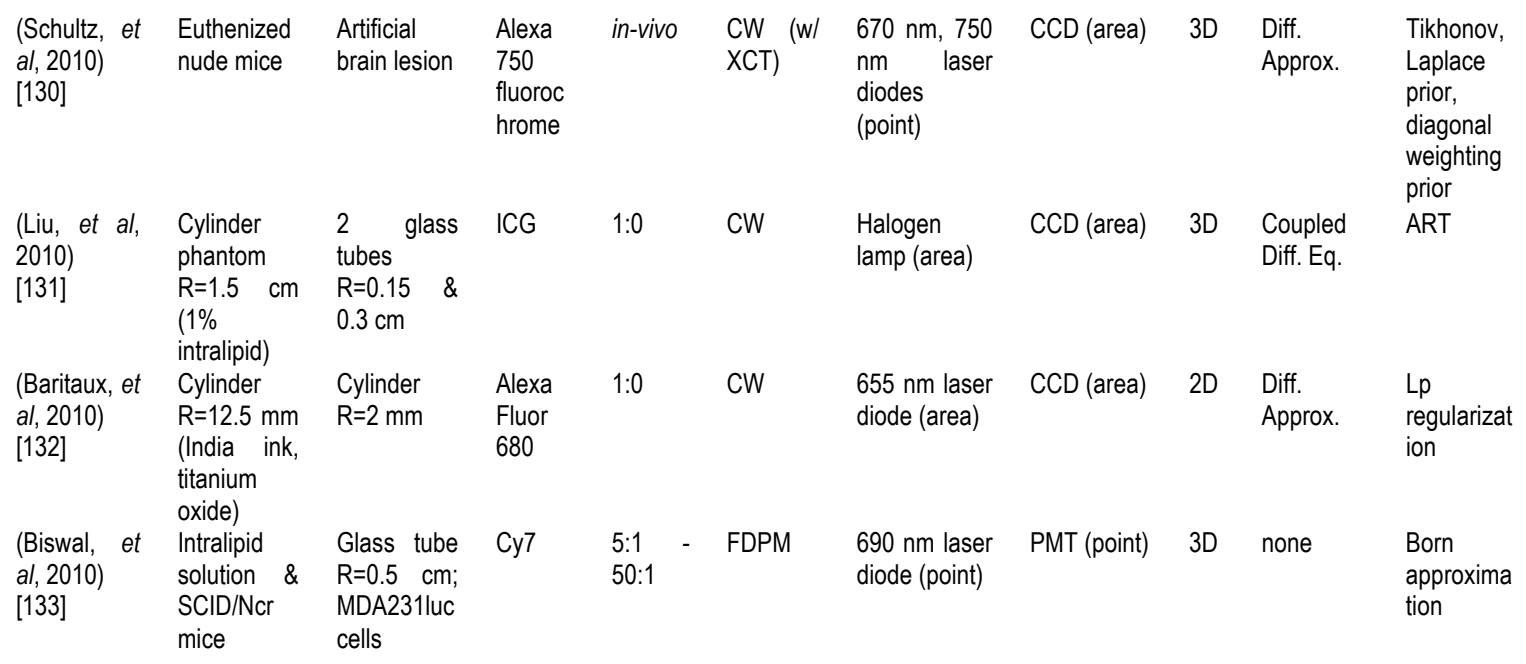

A research group from the University of Berlin used an algebraic reconstruction technique (ART) method to analyze DOT data and incorporated a noise-weighted back projection method.[124] This method has the advantages of: (i) allowing high cutoff frequencies to be chosen due to the algorithm's avoidance of significant distortions of the reconstructed image, and (ii) the ART algorithm uses a normalized image vector which improves the separation of absorption and scattering by rescaling to a dimensionless image vector with comparable size of the norm of both images. The algorithm was tested with experimental data from a breast phantom using measured time-domain diffuse reflectance and transmittance of excitation and fluorescence radiation. Images were collected with a fluorescent target embedded in the phantom with compressed-tissue geometry using three different source-detector geometries: (i) projection-shadow geometry; (ii) slab fan-beam geometry; (iii) slab reflection and transmission geometry. The results showed that collecting reflectance data at the excitation and fluorescent wavelength in addition to the transmitted data greatly improved the depth resolution. They concluded that scanning time-domain 
fluorescence mammography will be improved by the use of both reflectance and transmittance measurements from both sides of the compressed breast.

A group from Georgia Institute of Technology developed a reconstruction approach that uses a mean-square error algorithm and accounts for what they term as "tunneled light", i.e. the portion of light that passes through parts of the tissue which have lower absorption and hence has higher intensity than the portion of light that passes through the other parts of the tissue.[122] The reconstruction approach uses the strong spatial correlation between the excitation and emission images to estimate the tunneled components and gain a resulting image containing mostly signal due to the fluorescence. The resulting image is further refined using a coincidence mask (i.e. a map of the 'hot spots' that occur in both emission and excitation images). The algorithm was tested on experimental data from in vivo studies performed on a human colon adenocarcinoma xenograft tumor model. The results showed much stronger fluorescent signal from deeper tissues using this method. They concluded that identification, estimation, and correction of signal from tunneled light can improve image reconstruction in fluorescence tomography.

The research group from Harvard Medical School presented a Tikhonov based method for reconstruction of multimodal FMT-XCT or FMT-MRI images which uses information from within the data to get data-driven priors rather than the typical userdefined priors.[129] The first step compensates for the resolution differences between XCT (high resolution) and FMT (low resolution) by labeling each FMT voxel as a linear mixture of the different tissue types identified in the high-resolution image. The segment labels were used to construct a low dimensional inverse problem that gives a single intensity value for each anatomic area, and the values were used as parameters to define a spatially varying 
regularization term for the full resolution FMT problem. The algorithm was tested on experimental data collected from APP23 transgenic mice exhibiting amyloid- $\square$ plaques in the cortical region. The results showed that the method of using data driven priors generated improved reconstruction of the fluorescence in the cortical region.

A research group from Tsinghua University developed a method that uses principal component analysis (PCA) on a time-series of FDOT images in order to detect and visualize organs with different kinetics.[131] PCA is a method used to extract relevant features of a correlation matrix based on principal components, which express different kinetic behavior with high inter-correlations. PCA was performed on 6 frames from tomographic images (with zero mean) from dynamic FDOT images at the same slice. The principal component elements were used as weight factors to generate images containing different colors to represent regions with different kinetic behavior. The method was tested on simulated mouse data and experimental data from phantom studies. The results using the PCA method showed images that illustrated organs and functional structures that had different kinetic patterns. They concluded that the PCA method (i) provides a non-invasive method for measuring changes in organ function, (ii) enhances the resolution and specificity of FDOT through emphasis of differences in kinetic behavior, (iii) provides additional anatomical information without the use of multiple modalities, and (iv) is not subject to model-based restrictions since it is independent of any kinetic model.

A research group from the Swiss Federal Institute of Technology developed a reconstruction approach that uses what they term as $L_{p}$ regularization $(p \geq 1)$ to reconstruct fluorescence data using a matrix-free strategy in order to reduce computational costs.[132] They tested the sparsifying effect of using $\mathrm{L}_{1}$ regularization and compared it to $\mathrm{L}_{2}$ 
regularization (Tikhonov method). 2D reconstructions were performed using experimental phantom data. The results showed that the method improved the computation time and memory requirements since it does not require calculation of a system matrix, and the reconstructions obtained using the $L_{1}$-regularization displayed fewer artifacts and improved localization than those using a linear algorithm.

A research group from the University of Connecticut developed a Born approximation based reconstruction approach which separately estimates the structural parameters of a target such as target center (depth and spatial $x$ and $y$ locations) and size, as well as reconstructs the functional information using only optical based measurements.[133] The method was tested on data from phantoms and mice injected with VEGF/Cy7 (an angiogenesis tracer). The results showed the ability to reconstruct fluorescent targets with less than $25 \mathrm{nM}$ of contrast agent up to $2 \mathrm{~cm}$ deep in both homogeneous and heterogeneous backgrounds.

\subsection{INSTRUMENTATION FOR FLUORESCENCE TOMOGRAPHY}

One major challenge of fluorescence tomography from an instrument perspective is poor spatial resolution due to the highly scattering nature of biological tissue. Several research groups are currently developing instrumentation designed to improve the imaging ability of fluorescence tomography by combining the method with spatial prior information from other methods such as x-ray CT.

The research group from Dartmouth College developed a multi-channel spectrally resolved fluorescence optical tomography system within a clinical MRI for imaging of molecular targets in small animals.[121] The fiber-optic based NIR system was composed of 16 fiber bundles which each contain six detection fibers and one source fiber used to obtain 
transmission and emission NIR spectra. The emission light was spectrally fitted to separate the fluorescent signal from the background improving the accuracy and sensitivity of images with small concentration of fluorophores. The tissue morphology acquired from MRI was used as an imaging guide for the optical reconstruction. Results from phantom and small animal studies demonstrated the capability to image low fluorescent contrast (3.3:1) in 70 $\mathrm{mm}$ diameter tissue volumes using the spatial prior information from the MR images.

Another group from Dartmouth College designed a small animal imaging system which couples fluorescence tomography (FT) and x-ray microcomputed tomography (microCT).[126] The information from microCT was used as spatial prior information and overlays the FT image on the microCT image. The FT system uses a rotating gantry with a single source and and a fan-beam of detectors which rotate around the surface of the object for non-contact excitation and detection. Results from phantom studies demonstrated the ability of the system: (i) to allow maximum sensitivity in optical tomography with single photon counting, (ii) use a noncontact imaging configuration to accompany microCT, (iii) obtain fluorescence and transmission data in parallel, (iv) require only several minutes total for imaging time, and (v) perform imaging with multiple detectors in parallel.

A research group from the University of California, Irvine developed a unique, fully integrated tri-modality gantry-based system that combines FT, DOT, and x-ray CT (XCT). $\mathrm{XCT}$ was used to provide anatomical prior information and DOT was used to provide functional prior information of the heterogeneous optical background for the FT reconstructed images.[128] The optical system used point source (3 optical fibers) and area detection configuration for transmission based imaging. Results using the DOT prior information only demonstrated accurate localization of a fluorescent target within a 
heterogeneous phantom background; however, the concentration of the fluorophore was recovered with $70 \%$ error. Results using both the DOT and XCT prior information recovered the fluorophore with only $8 \%$ error.

A research group from the Technical University Munich designed a hybrid small animal imaging system which combines fluorescence tomography and x-ray computed tomography.[130] The $360^{\circ}$-projection free-space FT system uses point source (two optical fibers) and area detection configuration for transmission based imaging. Structural priors from the XCT images were incorporated into the optical reconstruction to allow improved imaging performance. Results demonstrated the ability of the system to reconstruct an artificial brain lesion within a euthanized nude mouse.

Most of the instrumentation for fluorescence tomography studies has been focused toward small animal imaging (especially multimodality imaging). In our Optical Imaging Laboratory we have developed a hand-held probe based optical imaging system and demonstrated 3D fluorescence tomography in clinically relevant sized tissue phantoms.[3] Extensive phantom studies demonstrated the ability of the device to reconstruct a fluorescent target $\left(0.45 \mathrm{~cm}^{3}\right)$ up to a depth of $2.5 \mathrm{~cm}$ under perfect uptake conditions.[127] A $1.0 \mathrm{~cm}$ deep fluorescent target was recovered under a target to background contrast as low as 25:1. The sensitivity and specificity of the device was estimated at $43 \%$ and $95 \%$, respectively, based on phantom studies. In vivo fluorescence tomography performed in human subjects using the hand-held optical imager is demonstrated in Chapter 13 below. 


\subsection{EXPERIMENTAL METHODS OF FLUORESCENCE TOMOGRAPHY}

Fluorescence tomography imaging methods face several challenges such as strong excitation light leakage which masks the weak fluorescent signal, limited depth recovery when using reflectance-based measurements, and limited spatial resolution. Several research groups have performed studies designed to improve the results obtained from fluorescence tomography by optimizing the experimental imaging methods.

A research group from Dartmouth College performed a study to optimize fluorescence tomography for sub-surface imaging of protoporphirin IX.[115] Phantom experiments were performed using a noncontact based imaging system to compare fluorescence remittance imaging (FRI) and sub-surface fluorescence diffuse optical tomography (FDOT) with the contrast agent protoporphirin IX. They found that tomographic imaging was able to produce a better contrast ratio, while simple planar fluorescence imaging produced better signal to noise ratio. They concluded that an optimal system for localizing embedded fluorescent regions should combine fluorescence reflectance imaging and sub-surface tomography in order to increase sensitivity and improve depth detection.

The same group performed another study to investigate the influence of tissue optical properties on the shape of near-infrared (NIR) fluorescence emission spectra propagating through centimeters of tissue-mimicking material.[123] Experimental studies were performed using liquid and gelatin-based phantoms and Lutetium Texaphyrin (LuTex) contrast agent. The NIRFAST model platform was used to calculate spectrally resolved emission spectra at discrete wavelengths and compared to experimentally measured spectra. The phantom results showed spectral distortion resulting in emission peak shifts of up to 60 
$\mathrm{nm}$ in wavelength which is analogous to beam hardening and softening in $\mathrm{x}$-ray tomography which causes image artifacts. They concluded that spectrally resolved detection strategies should be implemented in order to account for these effects and reduce error.

A group from Harvard University tested the ability of a small animal imaging system to perform complete-angle projection $\left(360^{\circ}\right)$ fluorescence tomography in vivo in mouse lung and brain tumors.[125] In both tumor models, the fluorescence was positively identified close to the tumor location with noncontact FT in all imaging experiments. They also applied fluorescence dataset normalization in order to correct for dynamic range variation in the resulting illumination through the tissue and inhomogeneity.

\subsection{CONCLUSION}

Fluorescence tomography is a promising technique for noninvasive functional imaging. However, there are several challenges that are currently being addressed by researchers. The challenges include strong excitation light leakage which masks the weak fluorescent signal, poor spatial resolution due to high scattering of the light in biological tissues, and mathematical complexity of image reconstruction due to the ill-posed nature of the inversion problem. This chapter reviewed the current (2008-present) efforts of different research groups to improve the experimental methods, reconstruction algorithm, and instrumentation used in performing fluorescence tomography. 


\section{CHAPTER 5}

\section{Past Work: Clinical Diffuse Optical Imaging in Human Subjects}

Testing of diffuse optical imaging technology in human tissues is integral for translation of the technology into the clinical setting. In the past decade, several research groups have evaluated the performance of diffuse optical imaging in human breast tissue using different methods and instrumentation.[134-152] A systematic review of diffuse optical imaging of the healthy and diseased breast was performed by Leff, et al in 2008.[134] This chapter summarizes the major findings by Leff et al and then describes significant clinical studies performed in 2008-2010.

Leff et al systematically reviewed clinical studies involving close to 2,000 females who underwent diffuse optical imaging of healthy and diseased breast tissue. The review focused on summarizing the studies to determine the ability of diffuse optical imaging to (i) provide information on the tissue composition of healthy breast tissue, (ii) detect breast lesions/abnormalities, and (iii) differentiate benign from malignant disease.

Several articles reported the use of diffuse optical methods for measuring the optical properties of healthy breast tissue, namely the absorption coefficient $\left(\mu_{\mathrm{a}}\right)$ and reduced scattering coefficient ${ }^{19}\left(\mu_{\mathrm{s}}{ }^{\prime}\right)$. A summary of the experimentally determined absorption and scattering properties at near-infrared wavelengths is given in Table 5.1. The global averages (highlighted in pink in Table 5.1) determined the absorption coefficient $\left(\mu_{2}\right)$ to be $0.04 \pm 0.02$ $\mathrm{cm}^{-1}$ at wavelengths between 600 and $800 \mathrm{~nm}$ and $0.05 \pm 0.03 \mathrm{~cm}^{-1}$ at wavelengths between 800 and $900 \mathrm{~nm}$. The global average reduced scattering coefficient $\left(\mu_{\mathrm{s}}{ }^{\prime}\right)$ was determined to be $10 \pm 5 \mathrm{~cm}^{-1}$ at wavelengths between 600 and $700 \mathrm{~nm}$ and $8 \pm 4 \mathrm{~cm}^{-1}$ at wavelengths

\footnotetext{
${ }^{19}$ The effective scattering coefficient which accounts for the anisotropy factor (cosine of the angle of scattering).
} 
between 700 and $900 \mathrm{~nm}$. The remarkable consistency in absorption and scattering properties between studies using various methods and instrumentation supports the ability of diffuse optical methods in providing information on the composition of healthy breast tissue.

Table 5.1: Summary of optical properties of healthy breast tissue from the literature. Adapted from Leff, 2008 [134]

\begin{tabular}{|c|c|c|c|c|c|c|}
\hline \multirow[t]{3}{*}{ References } & \multicolumn{6}{|c|}{ Optical properties of healthy breast tissue } \\
\hline & \multicolumn{3}{|l|}{$\mu_{\mathrm{a}}$} & \multicolumn{3}{|l|}{$\mu_{\mathrm{s}}$} \\
\hline & $600-700$ & $700-800$ & $>800$ & $\begin{array}{l}600- \\
700\end{array}$ & $\begin{array}{l}700- \\
800\end{array}$ & $>800$ \\
\hline $\begin{array}{c}\text { Grosenick } \\
{[135]}\end{array}$ & $0.04 \pm 0.01$ & $0.04 \pm 0.01$ & - & $12 \pm 2$ & $10 \pm 2$ & - \\
\hline Intes [136] & 0.05 & - & - & 11 & - & - \\
\hline Yates [137] & - & 0.01 & - & - & - & - \\
\hline Taroni [138] & - & $0.05 \pm 0.01$ & $0.05 \pm 0.01$ & - & $11 \pm 1$ & $10 \pm 1$ \\
\hline Suzuki [139] & - & $0.005 \pm 0.001$ & - & - & $1 \pm 0$ & - \\
\hline Taroni $[140]$ & - & - & - & - & - & - \\
\hline $\begin{array}{c}\text { Grosenick } \\
{[141]}\end{array}$ & $0.04 \pm 0.01$ & $0.04 \pm 0.01$ & $0.05 \pm 0.02$ & $11 \pm 1$ & $10 \pm 1$ & $8 \pm 0$ \\
\hline $\begin{array}{c}\text { Durduran } \\
\text { [142] }\end{array}$ & - & $0.04 \pm 0.003$ & $0.05 \pm 0.03$ & - & $9 \pm 0$ & $8 \pm 2$ \\
\hline $\begin{array}{l}\text { Spinelli } \\
{[143]}\end{array}$ & $0.05 \pm 0.01$ & $0.04 \pm 0.01$ & $0.10 \pm 0.07$ & $13 \pm 0$ & $11 \pm 2$ & $12 \pm 0$ \\
\hline Pogue [144] & $0.01 \pm 0.16$ & $0.05 \pm 0.04$ & $0.005 \pm 0.02$ & $1 \pm 0$ & $1 \pm 0$ & $1 \pm 0$ \\
\hline Poplack [145 & & $0.05 \pm 0.02 *$ & & & $12 \pm 2 *$ & \\
\hline $\begin{array}{l}\text { Global } \\
\text { average }\end{array}$ & 0.04 & 0.04 & 0.05 & 10 & 8 & 8 \\
\hline
\end{tabular}

Several research studies were reported which involved optical imaging of breast tissue containing a lesion or abnormality (either benign or malignant). In a total of 212 cases, 179 lesions were detectable by different diffuse optical methods. This data suggests a lesion detection rate of $85 \%$ for optical mammography based approaches. In order to detect the presence of abnormal tissue by diffuse optical methods, there must be a detectable 
difference in the tissue optical properties of healthy and abnormal tissue. Several studies focused on comparing total hemoglobin concentration $(\mathrm{HbT})$ and oxygen saturation $\left(\mathrm{SO}_{2}\right)$ in healthy and abnormal tissue. The results showed that healthy breast tissue had an average HbT of $21 \pm 6 \mu \mathrm{M} / 1$ and average $\mathrm{SO}_{2}$ of $68 \pm 5 \%$. The average $\mathrm{HbT}$ and $\mathrm{SO}_{2}$ of fibroadenoma was $54 \pm 13 \mu \mathrm{M} / 1$ and $69 \pm 3 \%$ respectively. The average $\mathrm{HbT}$ and $\mathrm{SO}_{2}$ of malignant disease was $65 \pm 34 \mu \mathrm{M} / 1$ and $66 \pm 24 \%$ respectively. The findings show that there is significant difference in the HbT of healthy and abnormal breast tissue, while there is no obvious difference in $\mathrm{SO}_{2}$ between healthy and abnormal breast tissue. The review pointed out that only one study reported on the sensitivity and specificity for detecting malignancy using spectroscopic information. Chance et al used a multiwavelength hand-held device to acquire data over six years from 166 patients with 44 confirmed malignancies. They reported the ability to distinguish cancerous from noncancerous breast tissue with a sensitivity of $96 \%$ and specificity of $93 \%$. Overall, the results reported from different research groups demonstrate potential of using diffuse optical methods to differentiate healthy and abnormal breast tissue.

Several research groups reported studies to distinguish benign and malignant breast lesions based on diffuse optical measurements of blood volume, saturation, or both. Several of the studies showed that the maximum and average HbT were significantly higher in malignant lesions than benign lesions. However, the results were from a relatively small pool of data and more extensive trials will be required to demonstrate conclusive results.

Overall, the reports summarized by Leff et al suggest that diffuse optical studies of healthy breast tissue show the breast to be nonuniform, and the composition of healthy breast tissue is modified due to physiological changes during the life of the female. Diffuse 
optical methods seem capable of distinguishing lesions from healthy tissue and demonstrated detection of $85 \%$ of lesions in breast tissue. Results suggest that diffuse optical methods have potential to distinguish benign and malignant lesions, but further studies and improvements in the technology are needed.

A summary of reported studies in clinical imaging of breast tissue using diffuse optical methods between the years 2008 and 2010 is given in Table 5.2.

Table 5.2: Summary of clinical diffuse optical imaging studies between 2008 and 2010 .

\begin{tabular}{|c|c|c|c|c|c|c|c|c|c|c|c|}
\hline \multirow[b]{2}{*}{$\begin{array}{l}\text { Refere } \\
\text { nce }\end{array}$} & \multirow[b]{2}{*}{$\begin{array}{l}\text { Instrum } \\
\text { entation }\end{array}$} & \multirow[b]{2}{*}{$\begin{array}{l}\text { Met } \\
\text { hod }\end{array}$} & \multicolumn{2}{|c|}{$\begin{array}{l}\text { Measurement } \\
\text { Geometry }\end{array}$} & \multirow[b]{2}{*}{ Source } & \multirow[b]{2}{*}{$\begin{array}{l}\text { Dete } \\
\text { ctor }\end{array}$} & \multirow{2}{*}{$\begin{array}{l}\text { Measurem } \\
\text { ent } \\
\text { Technique }\end{array}$} & \multirow{2}{*}{$\begin{array}{l}\text { Measurem } \\
\text { ent } \\
\text { Parameter }\end{array}$} & \multirow[b]{2}{*}{ Subject } & \multirow[b]{2}{*}{ Sens } & \multirow[b]{2}{*}{$\begin{array}{l}\text { Spec } \\
\text {. }\end{array}$} \\
\hline & & & $\begin{array}{l}\text { Point/Ar } \\
\text { ea }\end{array}$ & $\begin{array}{l}\text { Reflect/ } \\
\text { Trans }\end{array}$ & & & & & & & \\
\hline $\begin{array}{l}\text { You, } \\
\text { et. al., } \\
2010 \\
{[146]}\end{array}$ & $\begin{array}{l}\text { Hand- } \\
\text { held } \\
\text { imager } \\
\text { (NIR \& } \\
\text { US) }\end{array}$ & $\begin{array}{l}\text { DO } \\
\text { T \& } \\
\text { US }\end{array}$ & $\begin{array}{l}\text { Point -- } \\
\text { Subsurf } \\
\text { ace }\end{array}$ & Reflection & $\begin{array}{l}\text { Laser } \\
\text { diode }(785, \\
830 \mathrm{~nm})\end{array}$ & APD & FDPM & $\mathrm{THC}$ & $\begin{array}{l}198 \\
\text { patients } \\
\text { (benign \& } \\
\text { malignant) }\end{array}$ & $\begin{array}{r}83.9 \\
\%\end{array}$ & $\begin{array}{r}66.7 \\
\%\end{array}$ \\
\hline $\begin{array}{l}\text { Solim } \\
\text { an, et. } \\
\text { al., } \\
2010 \\
{[147]}\end{array}$ & $\begin{array}{l}\text { Optical } \\
\text { bed w/ } \\
\text { parallel } \\
\text { plates \& } \\
\text { matchin } \\
\text { g fluid }\end{array}$ & $\begin{array}{l}\text { DO } \\
\mathrm{S}\end{array}$ & $\begin{array}{l}\text { Point -- } \\
\text { Compre } \\
\text { ssed }\end{array}$ & $\begin{array}{l}\text { Transillumi } \\
\text { nation }\end{array}$ & $\begin{array}{l}\text { Laser } \\
\text { diodes } \\
(690,730, \\
780,830 \\
\mathrm{~nm})\end{array}$ & PMT & TDPM & $\begin{array}{l}\mathrm{HbO} 2, \mathrm{Hb}, \\
\text { \%water, } \\
\text { SP }\end{array}$ & $\begin{array}{l}10 \text { breast } \\
\text { cancer } \\
\text { patients }\end{array}$ & -- & -- \\
\hline $\begin{array}{l}\text { Choe, } \\
\text { et. al., } \\
2010 \\
{[148]}\end{array}$ & $\begin{array}{l}\text { Optical } \\
\text { bed w/ } \\
\text { parallel } \\
\text { plates \& } \\
\text { matchin } \\
\text { g fluid }\end{array}$ & $\begin{array}{l}\mathrm{DO} \\
\mathrm{T}\end{array}$ & $\begin{array}{l}\text { Point -- } \\
\text { Compre } \\
\text { ssed }\end{array}$ & $\begin{array}{l}\text { Transillumi } \\
\text { nation }\end{array}$ & $\begin{array}{l}\text { Laser } \\
\text { diodes } \\
(650,690 \text {, } \\
750,786 \text {, } \\
830,905 \\
\mathrm{~nm})\end{array}$ & $\begin{array}{l}\text { CCD } \\
\text { came } \\
\text { ra }\end{array}$ & $\begin{array}{l}\text { FDPM \& } \\
\text { CW }\end{array}$ & $\begin{array}{l}\mathrm{HbO} 2, \mathrm{Hb} \text {, } \\
\mathrm{THC}, \mathrm{SC}\end{array}$ & $\begin{array}{l}47 \text { patients } \\
\text { (benign \& } \\
\text { malignant) }\end{array}$ & $98 \%$ & $90 \%$ \\
\hline $\begin{array}{l}\text { Jiang, } \\
\text { et. al., } \\
2009 \\
{[149]}\end{array}$ & $\begin{array}{l}\text { Optical } \\
\text { bed w/ } \\
\text { ring }\end{array}$ & $\begin{array}{l}\text { DO } \\
\text { S }\end{array}$ & $\begin{array}{l}\text { Point -- } \\
\text { Circular }\end{array}$ & $\begin{array}{l}\text { Reflection } \\
\& \\
\text { transillumin } \\
\text { ation }\end{array}$ & $\begin{array}{l}\text { Laser } \\
\text { diodes } \\
(660-850 \\
\mathrm{nm})\end{array}$ & -- & FDPM & $\begin{array}{l}\mathrm{HbT}, \mathrm{SO} 2 \text {, } \\
\% \text { water, } \\
\text { SP }\end{array}$ & $\begin{array}{l}16 \text { cancer } \\
\text { patients }\end{array}$ & -- & -- \\
\hline $\begin{array}{l}\text { Kukret } \\
\text { i, et. } \\
\text { al., } \\
2010 \\
{[150]}\end{array}$ & $\begin{array}{l}\text { Hand- } \\
\text { held } \\
\text { imager }\end{array}$ & $\begin{array}{l}\text { DO } \\
\mathrm{S}\end{array}$ & $\begin{array}{l}\text { Point -- } \\
\text { Subsurf } \\
\text { ace }\end{array}$ & Reflection & $\begin{array}{l}\text { Laser } \\
\text { diodes } \\
(660,690, \\
780,808, \\
830 \mathrm{~nm}) \& \\
\text { tungsten- } \\
\text { halogen } \\
\text { lamp }\end{array}$ & APD & $\begin{array}{l}\text { FDPM \& } \\
\text { CW }\end{array}$ & $\begin{array}{l}\text { specific } \\
\text { tumor } \\
\text { component } \\
\text { s (STC) }\end{array}$ & $\begin{array}{l}60 \text { subjects } \\
\text { w/ } 22 \\
\text { malignant, } \\
18 \text { benign, } \\
21 \text { control }\end{array}$ & $91 \%$ & $94 \%$ \\
\hline $\begin{array}{l}\text { Stahel } \\
\text {, et. } \\
\text { al., } \\
2009 \\
{[151]}\end{array}$ & $\begin{array}{l}\text { Hand- } \\
\text { held } \\
\text { imager }\end{array}$ & $\begin{array}{l}\text { DO } \\
\text { S }\end{array}$ & $\begin{array}{l}\text { Point -- } \\
\text { Subsurf } \\
\text { ace }\end{array}$ & Reflection & $\begin{array}{l}\text { Laser } \\
\text { diodes } \\
(690,730 \text {, } \\
750,808 \\
870,920 \\
970 \mathrm{~nm})\end{array}$ & APD & FDPM & $\begin{array}{l}\mathrm{HbO} 2, \mathrm{Hb} \text {, } \\
\% \text { water, } \\
\text { SP }\end{array}$ & $\begin{array}{l}20 \text { healthy } \\
\text { subjects }\end{array}$ & -- & -- \\
\hline
\end{tabular}




\begin{tabular}{|c|c|c|c|c|c|c|c|c|c|}
\hline $\begin{array}{l}\text { van de } \\
\text { Ven, } \\
\text { et. al., } \\
2009\end{array}$ & $\begin{array}{l}\text { Optical } \\
\text { bed }\end{array}$ & $\begin{array}{l}\text { FD } \\
\text { OT }\end{array}$ & $\begin{array}{l}\text { Point -- } \\
\text { Circular }\end{array}$ & $\begin{array}{l}\text { Transillumi } \\
\text { nation }\end{array}$ & $\begin{array}{l}\text { lasers } \\
(690,730, \\
780,830 \\
n m)\end{array}$ & CW & $\begin{array}{l}\text { Omocyanin } \\
\text { e (external } \\
\text { contrast } \\
\text { agent) }\end{array}$ & $\begin{array}{l}12 \text { cancer } \\
\text { patients }\end{array}$ & -- \\
\hline
\end{tabular}

Stahel et al performed a study to compare the optical properties of breast tissue between females who use oral contraceptives to regulate their menstrual cycles and those who had spontaneous menstrual cycles.[151] The study was performed using a multiwavelength frequency-domain hand-held optical imager containing a single source and detector fiber pair. The results of blood volume and oxygenation measurements showed that the breast perfusion, water content, and scattering power in females using oral contraceptives was nearly constant during the menstrual cycle and tended to vary in females with spontaneous menstrual cycles, while oxygen saturation tended to remain stable in both groups. This study further confirms the ability of diffuse optical methods to be used to gain information about healthy breast tissue.

Three groups reported studies using diffuse optical methods to distinguish benign and malignant breast lesions. Choe et al used an optical bed with parallel-plate imaging geometry to measure oxy-, deoxy-, and total hemoglobin concentration, along with blood oxygen saturation and tissue scattering in 47 subjects with 51 lesions.[148] 95\% of the malignant lesions were invasive ductal carcinoma; of the non-malignant lesions, $40 \%$ were fibroadenoma, 30\% were cysts, 30\% were lobular carcinoma in situ, and 10\% were fibrocystic disease. The results showed higher total hemoglobin concentration (THC) and scattering in malignant lesions than in the surrounding tissue. Cysts showed lower scattering than the background. Fibroadenomas showed weak contrast in THC and scattering. The tumor-tonormal ratios of all parameters were close to unity in the benign lesions, whereas they were 
significantly different from unity in the malignant cases. Kukreti et al used data collected with a combined FD-CW hand-held optical imager with dual source and detector pairs.[150] They used data from 22 malignant and 18 benign lesions to retrospectively determine if benign and malignant lesions could be distinguished using near-infrared spectroscopy. Results showed a sensitivity of $91 \%$ and specificity of $94 \%$. You et al used a combined nearinfrared and ultrasound based hand-held imager to measure THC in 198 females with 214 lesions (96 benign and 118 malignant).[146] The average THC in benign lesions was $125.5 \pm$ $83.4 \mu \mathrm{M} / 1$ while in malignant lesions it increased to $222.2 \pm 87.2 \mu \mathrm{M} / 1$. Using a cutoff of $140 \mu \mathrm{M} / 1$ they were able to detect malignant lesions with a sensitivity of $83 \%$ and specificity of $66.7 \%$. The results from these three studies show continued promise of the ability of diffuse optical methods to distinguish benign and malignant lesions which has potential to reduce the number of surgical biopsies in women with breast lesions.

An additional aspect where diffuse optical methods have been applied for breast imaging is to monitor the response to neoadjuvant chemotherapy. Jiang et al performed a study to evaluate two methods used to summarize diffuse optical data by region of interest analysis to compare complete and incomplete response to neoadjuvant chemotherapy in patients with locally advanced breast cancer.[149] In seven cases of subject data, diffuse optical spectroscopy (DOS) measurements of total hemoglobin concentration, blood oxygen saturation, water fraction, optical scattering amplitude, and scattering power were analyzed using both fixed- and variable-size region of interest (ROI). Results showed that only the measurements of total hemoglobin demonstrated significant difference in the complete and incomplete response outcomes. Soliman et al used diffuse optical methods to measure tissue concentration of deoxyhemoglobin $(\mathrm{Hb})$, oxyhemoglobin $\left(\mathrm{HBO}_{2}\right)$, percent water $(\%$ water $)$, 
and scattering power (SP) in ten patients undergoing neoadjuvant chemotherapy for locally advanced breast cancer.[147] The results showed significant difference in all four parameters between responders and nonresponders to the the therapy. The studies performed by these two groups demonstrate the potential role of diffuse optical imaging in monitoring response to neoadjuvant chemotherapy in breast cancer patients.

External contrast agents can be used in order to improve the detection and characterization of breast lesions by diffuse optical methods, especially for smaller-size tumors. Van de Ven et al performed a study to evaluate a novel fluorescent imaging agent, Omocianine, in 11 women suspected of breast cancer.[152] Four different dosage levels were used, the smallest and medium doses were each used in three cases and the two highest doses were used in five cases. The results showed the ability to detect two out of three tumors using the lowest dose and three out of three tumors using the medium dose. None of the five tumors were detected using the two highest doses. These results demonstrate the potential of using Omocianine as a fluorescent contrast agent to detect lesions using low doses of the agent.

Diffuse optical imaging of breast tissues has been explored by several research groups in the clinical setting using various methods and instrumentation. Studies show the potential of diffuse optical methods to gain information in healthy breast tissue, distinguish abnormal tissue from healthy background tissue, distinguish benign and malignant tumors, and monitor response to neoadjuvant chemotherapy. Additionally, use of external contrast agents can improve imaging ability of diffuse optical methods by increasing the contrast.

Clinical trial in human subjects is an essential and defining stage in the development of new imaging technology. Hand-held optical imagers provide a promising avenue toward 
rapid translation of the optical imaging technology to the clinical setting. The overall objective of the dissertation is toward the clinical translation of our hand-held optical imager, which is unique in its ability to perform 3D tomography. Various studies were performed in tissue phantoms, in vitro, and in vivo toward this objective. The next chapter describes the instrumentation and data analysis procedures for the studies performed toward clinical translation of the hand-held optical imager developed in our Optical Imaging Laboratory. 


\section{CHAPTER 6}

\section{Instrumentation and Data Analysis}

\subsection{INTRODUCTION}

A hand-held optical imaging device has been designed and developed in our Optical Imaging Laboratory with the following unique features: (i) flexibility to image different tissue curvatures $\left(0^{\circ}-45^{\circ}\right)$ via a geometrically adaptive probe head; (ii) ability to simultaneously illuminate (at 6 point locations) and collect NIR signals (at 165 point locations) towards rapid data acquisition; (iii) portability and comfort from the hand-held design with maximal patient comfort [3]; and (iv) coregistration facilities to enable 3D tomographic imaging in complex tissue geometries [153].

Previously, experiments were performed to demonstrate $3 \mathrm{D}$ fluorescence tomography using the hand-held based optical device in slab tissue phantoms [3]. In order to perform 3D tomography, the image collected with the hand-held device must be coregistered to the geometry of the phantom being imaged. For the past studies, the images collected using the probe were manually coregistered to the simple slab phantom geometry at the known location on the phantom surface. In the current research, automated coregistration capabilities are implemented to facilitate translation of the device towards clinical studies in order to perform 3D tomography in curved (in vivo) tissue geometries. Experimental studies are performed using the hand-held based optical imaging system on tissue phantoms, in vitro, and in vivo with human subjects. This chapter describes the instrumentation used to collect continuous-wave and frequency-domain based measurements (Section 6.2), the principle of continuous-wave and frequency-domain based 
optical imaging (Section 6.3), the experimental protocol for data acquisition in phantom and in vitro studies (Section 6.4), and the automated coregistered imaging process (Section 6.5).

\subsection{OPTICAL IMAGING SYSTEM}

The three major components of the imaging system are the hand-held probe, the laser diode source, and the intensified charge-coupled device (ICCD) detector. The instrumentation is developed such that it can acquire both continuous wave $(\mathrm{CW})$ based and frequency-domain photon migration (FDPM) based optical measurements as required.

The hand-held probe head is designed with unique features in that it is flexible to contour to different tissue curvatures, it has a large imaging area $\left(4 \mathrm{x} 9 \mathrm{~cm}^{2}\right)$ for large volume interrogation, and it uses simultaneous illumination and detection for rapid data acquisition. The hand-held probe head is shown in Figure 6.1. The six red dots show the location of six source fibers and the black dots are the 165 detector fibers. The probe is divided into three sections and each of the side sections is capable of curving up to $45^{\circ}$.

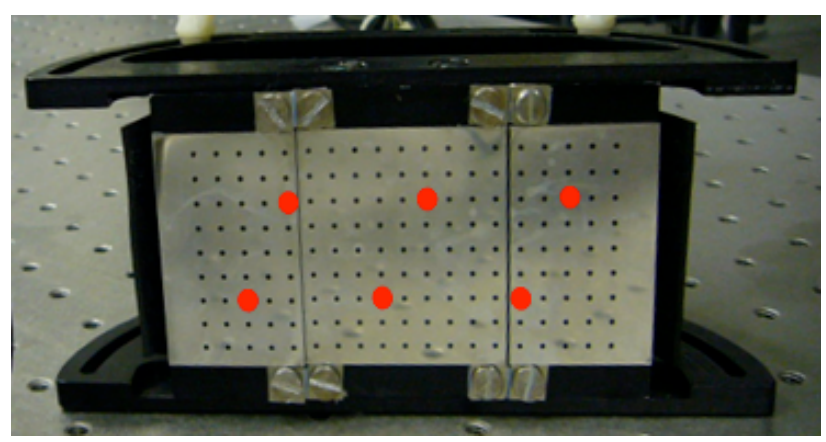

Figure 6.1 The actual hand-held probe face showing the source-detector configuration. The large red dots represent the six source fiber locations and all other small holes are the 165 detector fiber locations.

At the source end, a single 785-nm laser diode source $530 \mathrm{~mW}$, HPD1005-9MM, Intense Ltd., North Brunswick, NJ) is split into six illuminating fibers using a custom-built 
collimator-diffuser package. The laser source is coupled to the handheld probe as shown in Figure 6.2. For FDPM measurements, the source end is modulated using a frequency synthesizer (HP 8656B, Hayward, CA) and RF amplifier (ENI500L, ENI Inc., Rochester, NY) as shown in Figure 6.3.

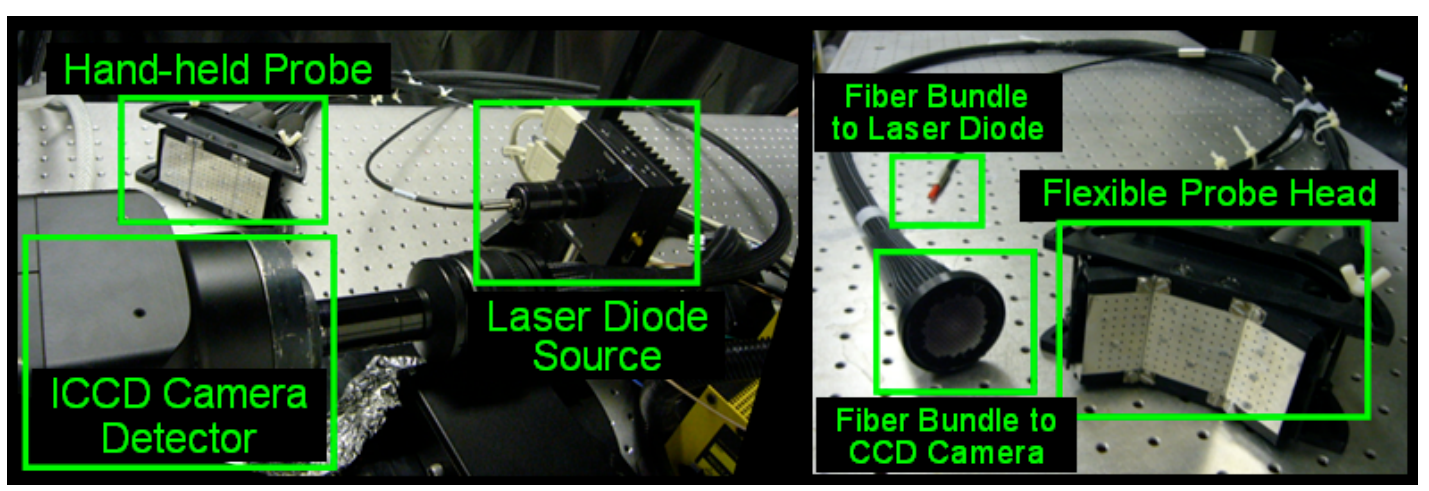

Figure 6.2 Hand-held probe based optical imaging system showing the hand-held probe is fiberoptically coupled to the laser source and ICCD camera (left). The probe face is flexible to contour to different tissue curvatures (right).

At the detector end, a gain modulated image intensifier (FS9910, ITT Night Vision, VA) is optically coupled to the CCD camera (PI-SCX 7495-0002, Roper Scientific, Trenton, NJ). For FDPM measurements, the image intensifier is modulated using a frequency synthesizer (PTS 310, Programmed Test Sources Inc., Littleton, MA) and RF amplifier (ENI503L, ENI Inc., Rochester, NY) as shown in Figure 6.3. The two frequency synthesizers at the source and detector end are phase locked and operated at the same frequency $(100 \mathrm{MHz})$. The output intensity from the ICCD camera is sensitive to the phase delay and upon introducing a phase shift from 0 to $2 \square$, phase-sensitive images are collected from which measurements can be extracted in terms of amplitude (AC) and phase ( $\square$ ) of the modulated signal. 


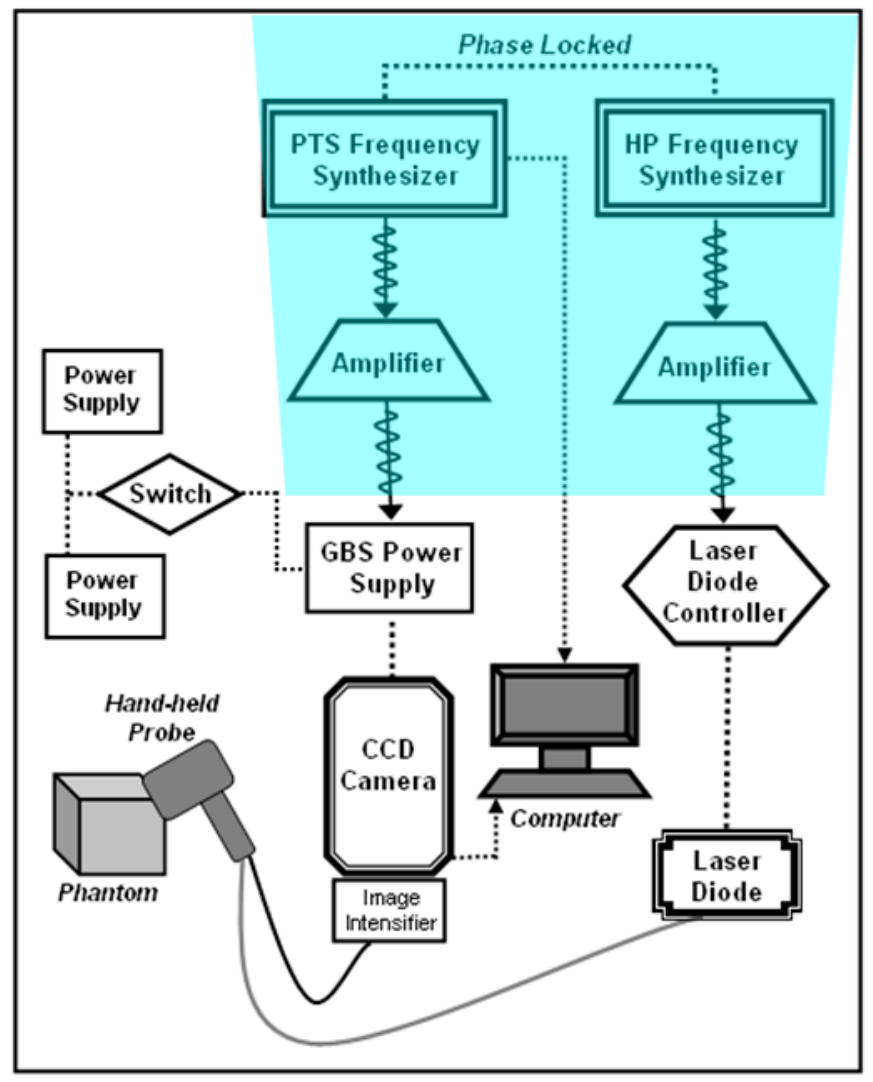

Figure 6.3 Schematic of the instrumentation for the hand-held optical imager. The CW based imaging system is shown in white and the additional components for FDPM based imaging are highlighted in blue.

The CCD camera acquires steady-state phase sensitive images at 32 phase delays between 0 and $2 \square$. The exposure time for the CCD camera is 0.2 seconds (s) for a single image (in all experiments) and each collected image represents the average signal from 5 repetitions. The exposure time was kept small enough to allow rapid image acquisition and using more than 5 repetitions was previously determined not to improve the result.[154] The image is acquired in $1024 \times 1024$ pixels and is hardware binned to $128 \times 128$ pixels for improved signal to noise ratio and faster imaging time. The raw image is post-processed in MATLAB software developed in house to get the DC values (for CW imaging) at each detector location on the probe and displayed as a 2D contour plot. For FDPM imaging, the 
amplitude (AC) and phase $\square$ ) information is extracted using fast Fourier transforms (FFT), and the final image is presented as a $2 \mathrm{D}$ surface contour plot of the $\mathrm{AC}$ or phase values at each of the 165 detector points of the probe.

When operated in continuous wave mode (Figure 6.3, white portion), the laser light is emitted at a constant intensity and travels to the tissue via optical fibers connected at the probe face. The light passes from the probe into the phantom or tissue and becomes attenuated due to the effect of the absorption and scattering properties of the medium. The reflected light is collected at the tissue surface by the optical fibers in the probe and carried to the detector. Different types of filters are used depending on the type of study. For absorption based studies a neutral density filter is used to uniformly attenuate the collected signal. For fluorescence based studies two band pass interference filters of blocking OD 4 and 6 (HRF-830.0, blocking OD 6 and F-830.0, blocking OD 4, CVILaser, Albuquerque, $\mathrm{NM}$ ) are used to select the weak 830nm fluorescent signal and reject the $785 \mathrm{~nm}$ excitation signal. The filtered signal reaches the image intensifier at the photocathode and is converted into electrons. The electrical signal is amplified at the micro-channel plate (MCP) according to the manual gain setting on the power supply of the image intensifier. The amplified signal then encounters the phosphor screen where it is converted back to a light signal and imaged onto the CCD camera. The raw image is a $1024 \times 1024$ distribution of intensity signal from each of the 165 detector fibers in the bundle. The $1024 \times 1024$ image is hardware-binned to $128 \times 128$ to reduce noise and processing time. Post-processing MATLAB software developed in house is then used to back calculate the location of each detection point on the hand-held probe face and interpolated to generate a $2 \mathrm{D}$ surface contour plot. Frequencydomain based optical imaging involves the use of a modulated laser signal. In our hand-held 
based optical imaging system, the incident light is continuously produced and the intensity is sinusoidally modulated via a frequency synthesizer at $100 \mathrm{MHz}$. As the intensity-modulated light passes from the hand-held probe into the tissue, the intensity of the signal becomes attenuated and the phase of the modulation altered according to the optical properties of the tissue. The attenuated and phase-shifted signal is then collected from the tissue surface and the detected signal is modulated at the same frequency. The fast Fourier transform is used to extract the intensity and phase information to generate separate $2 \mathrm{D}$ contour plots which in turn provide information about the optical properties within the tissue.

\subsection{ACQUISITION OF OPTICAL DATA WITH THE HAND-HELD IMAGER}

Two-dimensional (2D) diffuse optical and fluorescence-enhanced imaging was performed, using the hand-held device operated in the CW-mode, on tissue phantoms, and in vitro. Spherical acrylic targets (of different sizes) filled with $0.02-0.08 \%$ India ink as an absorbing contrast agent (for diffuse optical studies) or $1 \mu \mathrm{M}$ ICG fluorescent contrast agent (for fluorescence-enhanced studies) were used to mimic a tumor in experiments with tissue phantoms, in vitro, and healthy human subjects. The probe was placed in full contact with the phantom or tissue surface as shown in Figure 6.4 and CW images of the absorption or fluorescent intensity were acquired in close to real-time ( $\sim 2 \mathrm{sec}$ delay). The raw intensity images at the ICCD camera end were acquired in 1 -sec $(0.2 \mathrm{sec}$ exposure time $\times 5$ repetitions). These images were post-processed ( $\sim 1 \mathrm{sec})$ using in-house developed Matlab codes (Section 6.4) in order to acquire the final 2D surface contour plots of fluorescence intensity distribution of the imaged surface. The entire data acquisition and post-processing was automated such that close to real-time ( $\sim 2$ sec delay) imaging is possible. For frequency-domain imaging, phase-sensitive images were collected with a $\sim 2$-minute imaging 
time. The 2D surface contour plots of fluorescence intensity signal may or may not differentiate the target from the tissue phantom background, based on the target and background optical properties.

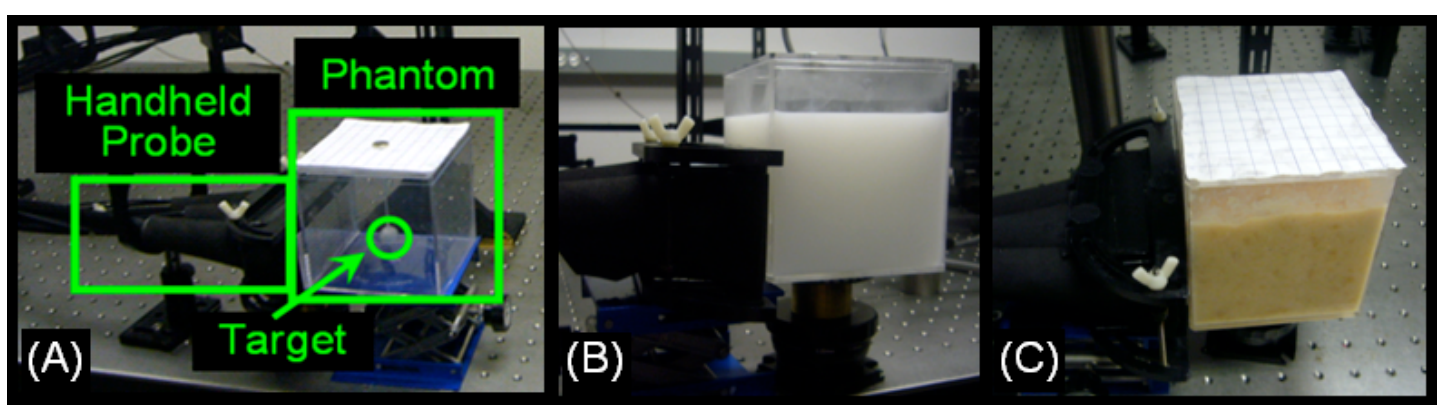

Figure 6.4 Experimental set-up for tissue phantom, in vitro, and in vivo studies: (A) empty phantom showing target inclusion and placement of probe, (B) tissue slab phantom with 1\% Liposyn solution for uniform scattering in the background, and (C) in vitro slab phantom with heterogeneous scattering in the background.

\subsection{ELIMINATION OF EXCITATION LIGHT LEAKAGE (SUBTRACTION BASED POST-PROCESSING)}

During fluorescence optical imaging, the output signal at the tissue surface is a mixture of fluorescence signal and the attenuated incident NIR (i.e. excitation) signal. This fluorescence signal is filtered from the strong excitation signal (3-4 orders of magnitude higher) using appropriate optical (bandpass) filters and imaged by the detector. However, the filters are not capable of $100 \%$ rejection of the excitation light, causing excitation leakage and contamination of the fluorescent signal. Hence, a second-level of post-processing is carried out to subtract a background non-fluorescing image from the final fluorescence image plots for each experimental case, in order to account for the excitation leakage. Initially, optical measurements were acquired prior to placing the fluorescent target in the phantom, in an attempt to represent the excitation leakage (or background noise). These (background noise) measurements were subtracted from the fluorescence optical measurements obtained from 
experimental cases that included fluorescent targets, in order to effectively eliminate the signal from the excitation source light. In the clinical setting involving actual diseased tissues (unlike the simulated fluorescent targets in the current study), this could be accomplished by acquiring image(s) of the tissue before and after the contrast agent (e.g. ICG) injection. The non-fluorescent image(s) acquired prior to ICG injection will in turn be subtracted from the fluorescent images acquired after ICG injection, in order to account for the background noise. Another method is to collect an image from both breasts and subtract the image of the ipsilateral (tumor-containing) breast from the image of the contralateral (non-tumorcontaining) breast. This was the method applied in the human subject studies with breast cancer patients (Chapter 14).

A single image acquisition consists of collecting an image with target ( $2 \mathrm{sec})$, collecting a background image $(\sim 2 \mathrm{sec})$ and subtracting the two images $(\sim 1 \mathrm{sec})$. The subtracted 2D optical images are generated rapidly $(<5 \mathrm{sec})$, making the entire process a fast 2D surface imaging technique.

\subsection{AUTOMATED COREGISTERED IMAGING PROCESS}

In order to perform 3D tomography using a hand-held optical imager, it is necessary to coregister the $2 \mathrm{D}$ image at the appropriate location on the tissue geometry. For the past tomography studies with cubical tissue phantoms, the images were manually coregistered at the known locations on the simple cubicle geometries. In order to perform 3D tomography in human tissue with varied complex geometries, it is necessary to track the probe location in real time and automatically coregister the image at the tissue location. To this end, coregistered imaging facilities developed by a master's student in our lab have been implemented with our hand-held based optical imaging device. 
Coregistered imaging involves the alignment of two or more images (planar or volumetric) in a single coordinate space. Herein, an intramodality ${ }^{20}$ coregistration method (similar to 3D ultrasound imaging) is used to acquire 3D coregistered surface images from 2D surface data collected using the hand-held device. These 3D surface images will in turn be used towards 3D tomographic analysis. In coregistered imaging, the position and orientation of the probe is tracked with respect to the tissue or phantom surface being imaged. This tracked 3D positional information is then used to accurately position the acquired optical images onto the tissue geometry.

Tracking of the probe position is achieved using an acoustic-based 3D tracker (Logitech Inc., Fremont, CA) which consists of a transmitter (positioned $\sim 2$ feet above the hand-held probe), receiver (implemented onto the probe head), and a processing unit. The position and orientation of the receiver (probe head) is tracked in real-time in reference to the transmitter. The probe's position is acquired by the computer with six degrees of freedom ( $\mathrm{x}, \mathrm{y}, \mathrm{z}$, pitch, yaw, and roll $^{21}$ ) using MATLAB software developed in house and interfaced to the processing unit tracking software. The tracked probe's position and orientation are displayed on the computer screen as position coordinates angles of rotation.

Coregistered imaging of tissue/phantoms is implemented as a three-stage process (see Figure 6.5).

Stage 1: The special 3D location of the probe is determined and the probe's position and orientation is tracked in real-time with respect to a discretized mesh of the phantom or tissue geometry (steps 1-4 in Figure 6.5).

\footnotetext{
${ }^{20}$ Two types of coregistration techniques that exist include intermodality and intramodality. Intermodality coregistration combines images of different modalities (e.g. CT/MRI, SPECT/MRI, optical/MRI, etc.), whereas intramodality coregistration combines images from the same modality (e.g. 3D ultrasound imaging).

21 Pitch, yaw, and roll refer to the tracked object's rotational orientation in reference to the $\mathrm{x}-, \mathrm{y}-$, and $\mathrm{z}$-axis respectively.
} 


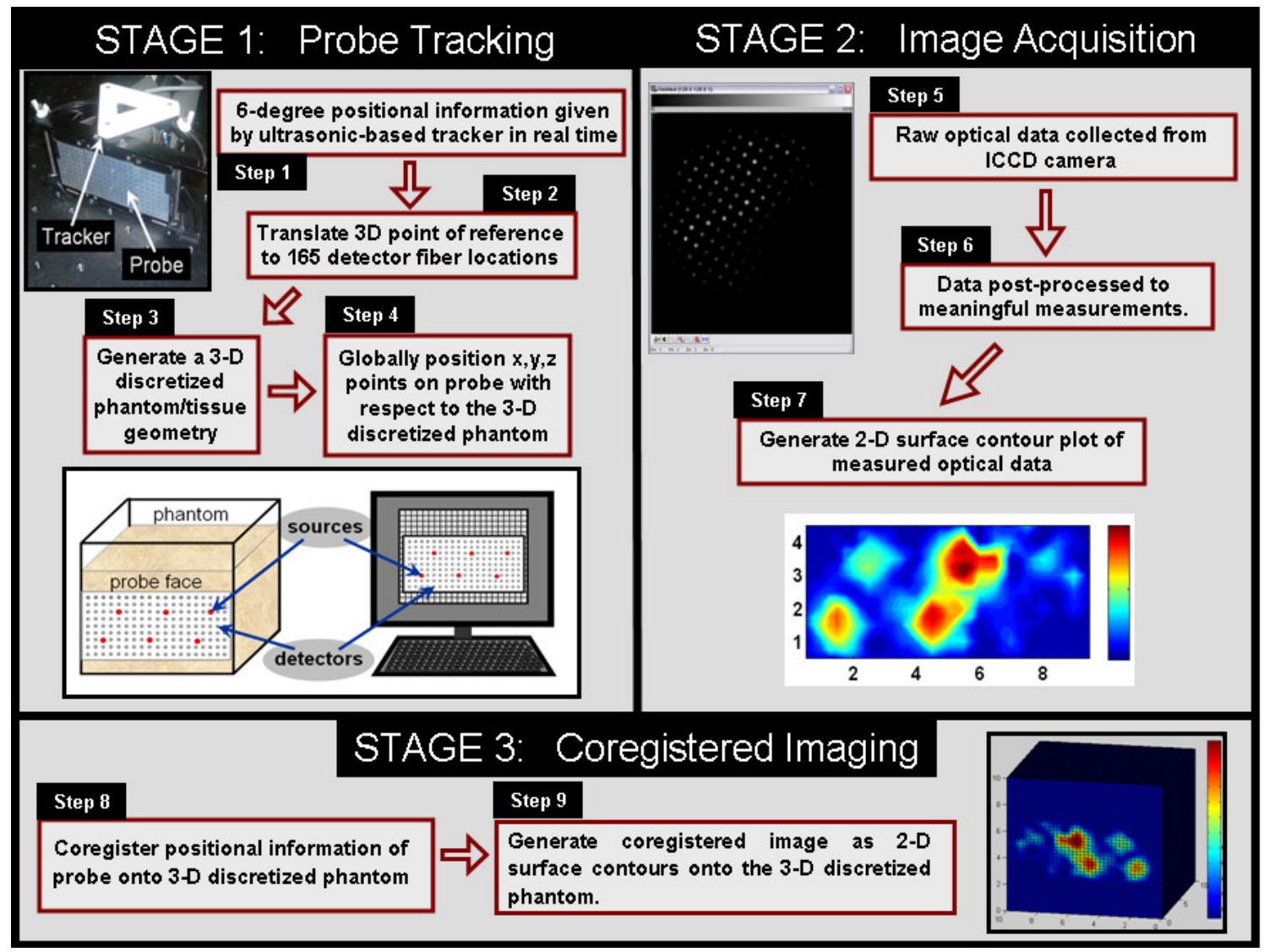

Figure 6.5 Flowchart of the coregistered imaging process, implemented as automated coregistration software during optical imaging studies.

Stage 2: The hand-held probe based imaging system is used to acquire raw data, i.e. optical signals of intensity (step 5 of Figure 6.5). The raw data is post-processed (step 6 of Figure 6.5) using MATLAB software developed in house as described in section 4.1 to generate and display the image as a $2 \mathrm{D}$ surface contour plot (step 7 of Figure 6.5). The average time to acquire the raw data and display the post-processed images $(2 \mathrm{D}$ contour plots $)$ is $\sim 2 \mathrm{sec}(0.2 \mathrm{sec}$ exposure time for each of the 5 repeated measurements, and $\sim 1 \mathrm{sec}$ for data post-processing to obtain 2D surface contour plots). 
Stage 3: Image coregistration is performed using tracking/coregistration interfacing software developed in house in MATLAB/LabVIEW. The optical data from the 2D surface contour plot is coregistered onto the $3 \mathrm{D}$ discretized phantom at the probe location using the positional information of the entire probe head (i.e. all source and detector point locations) during each scan. The entire coregistered imaging process is described in detail in reference [153].

The current version of the coregistration software is designed to work with phantoms with known position/orientation, and does not take into account any motion of the phantom or tissue being imaged. In the future, this can be remedied by adding tracking abilities to the phantom (or subject) being imaged, thereby allowing location and orientation of the phantom/tissue to be automatically known and updated in real-time. The use of additional trackers is currently being implemented by a master's student in our laboratory.

\subsection{CONCLUSION}

A CW and FDPM based hand-held optical imaging system has been developed in our laboratory for coregistered imaging and 3D tomography of breast tissue. This chapter detailed the instrumentation and data analysis procedure for the experimental studies to perform fast $2 \mathrm{D}$ imaging. The next chapter will describe the AEKF based reconstruction algorithm used to perform diffuse optical and fluorescence-enhanced diffuse optical tomography using our hand-held based optical imaging device. 


\section{CHAPTER 7}

\section{Fluorescence-enhanced Diffuse Optical Tomography}

\subsection{INTRODUCTION}

Three dimensional (3D) optical tomography is used to reconstruct the $3 \mathrm{D}$ volume and location of the tumor within the tissue. Measurements collected at the surface of the tissue are used to mathematically reconstruct the optical property distribution at discrete points within the tissue volume. The optical properties of interest in biological tissue are absorption and scattering. There are two types of mathematical problems addressed in tomography: the forward problem and the inverse problem. The forward problem is used to simulate light propagation in tissue of known optical property distribution. The inverse problem is used to reconstruct the optical properties within the tissue given a set of experimentally measured values (fluence) at the tissue surface. In each mathematical formulation, the tissue volume is discretized and the optical properties are calculated at each discrete location. This chapter will describe the mathematical model of light propagation in tissue, the forward and inverse problems, and the AEKF reconstruction algorithm used to perform 3D tomography.

\subsection{LIGHT PROPAGATION IN BIOLOGICAL TISSUES}

The propagation of light through a highly scattering medium is often modeled using the diffusion approximation of the radiative transport equation. The coupled diffusion equations for the case of fluorescence-enhanced imaging in the frequency domain are [104]

$$
-\nabla \cdot\left[D_{x} \nabla \Phi_{x}\right]+\left[\mu_{a x}+\frac{i \omega}{c_{x}}\right] \Phi_{x}=S \delta\left(\mathrm{r}-\mathrm{r}_{\mathrm{s}}\right)
$$




$$
-\nabla \cdot\left[D_{m} \nabla \Phi_{m}\right]+\left[\mu_{a m}+\frac{i \omega}{c_{m}}\right] \Phi_{m}=q \mu_{a x \rightarrow m}\left[\frac{1}{1-i \omega \tau}\right] \Phi_{x}
$$

where the subscripts $x$ and $m$ are used to represent the excitation and emission wavelengths of light respectively. In these equations, $\square$ is used to represent the amplitude of the fluence signal $\mu_{\mathrm{a}}$ and $\mu_{\mathrm{s}}$ represent the absorption and scattering coefficients, $q$ and $\square$ are the quantum efficiency and fluorescent lifetime of the fluorophore, $c$ is the velocity of light, $\square$ is the modulation frequency, and $\mathbf{r}$ is the positional vector. $\mathrm{D}$ is the diffusion coefficient given by:

$$
D_{x, m}=\frac{1}{3\left(\mu_{a x, m}+\mu_{s x, m}(1-g)\right)}
$$

where $g$ is the anisotropy factor. The partial current boundary condition is applied which assumes that once a photon leaves the tissue it does not return. It is expressed by

$$
\Phi_{x, m}+2 \gamma D_{x, m}\left[\frac{d \Phi_{x, m}}{d n}\right]=0
$$

where $\square$ is the index-mismatch parameter, which is a function of the effective refractive index, and $n$ is the normal to the surface.

The diffusion approximation of the radiative transport equation is applied in the forward and inverse problems to model light propagation in tissue and to reconstruct the internal distribution of tissue optical properties using surface measurements.

\subsection{FORWARD PROBLEM}

The forward problem in optical imaging is used to predict light transport in biological tissue during simulations. The problem is stated as follows: Given a distribution of light sources 
on the boundary of an object and a distribution of tissue parameters within the object, find the resulting measurements that would occur at the surface. Figure 7.1 illustrates the forward and inverse problems. In the forward problem (left), the parameters $\mu_{\mathrm{a}}$ and $\mu_{\mathrm{s}}$ ' are known within the tissue and the propagation of light to the boundaries is predicted. In biomedical optical imaging, the forward problem is typically solved using the diffusion approximation to the radiative transport equation described in section 7.2. The diffusion equation is solved numerically using a finite difference or finite element based approach. For the studies described herein, a Galerkin based finite element approach was used to solve the forward problem.

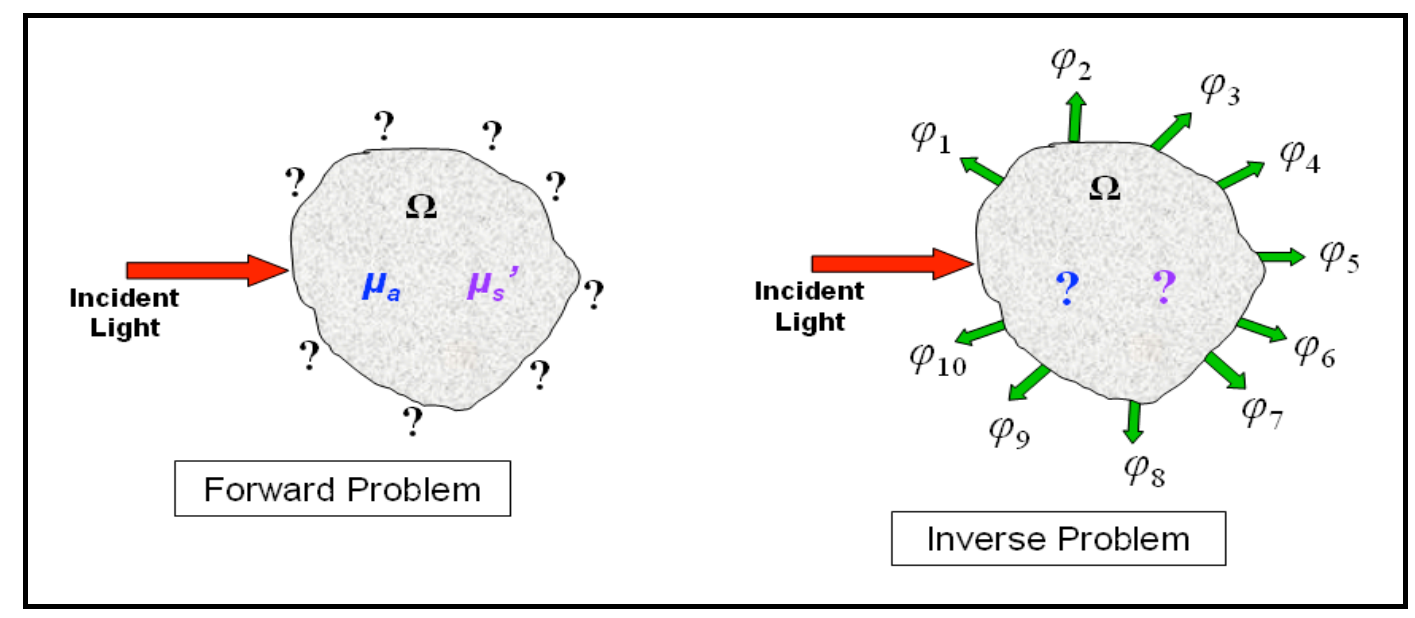

Figure 7.1 Illustration of the forward and inverse problem in optical tomography. In the forward problem, the optical property distribution is known and used to predict the measurements that would occur at the surface of the tissue. In the inverse problem, the measurements obtained experimentally at the surface are used to reconstruct the unknown optical property distribution within the tissue.

\subsection{INVERSE PROBLEM}

The inverse problem in optical imaging is to reconstruct the unknown optical properties within a tissue given a set of measurements at the boundary. The problem is stated as follows: Given a distribution of light sources and a distribution of measurements on the 
surface, calculate the tissue parameter distribution within the tissue. The inverse problem is illustrated in Figure 7.1 (right) where a set of measurements of fluence $(\square$ i) is collected at the boundary, and the unknown internal properties of the given tissue are mathematically reconstructed (in this case, $\mu_{\mathrm{a}}$ and $\mu_{\mathrm{s}}{ }^{\prime}$ ). In the case of the inverse problem, the number of unknowns is much greater than the total number of surface measurements acquired and the problem is to solve an underdetermined system of linear equations. The solutions are nonunique and inconsistent making the problem 'ill-posed'

\subsection{APPROXIMATE EXTENDED KALMAN FILTER (AEKF) BASED RECONSTRUCTION ALGORITHM}

Optical tomography has been performed using different algorithms such as Algebraic Reconstruction Techniques (ART) [125], NIRFAST [126], and Born approximation [133]. For the tomography studies performed herein, a Bayesian reconstruction technique was used which is an approximate extended Kalman filter (AEKF).[104] The Kalman filter is a linear state Bayesian estimator that is used in process control in order to estimate a state recursively, using the previous observations. The filter yields estimates of true measurement values and the corresponding calculated values by the following process: (i) predict a value, (ii) estimate the uncertainty of the predicted value, (iii) compute a weighted average of the predicted value and the measured value. The estimator determines the mean value of the conditional probability function of the unknown parameters using given measurements, assuming that the state variables and observations are normally distributed random variables. This Bayesian estimator was chosen due to the following advantages: (i) the updates in each iteration are weighted and the matrix inversion is regularized using measurement error (between repeated measurements) and model error (which results from simplifications of the 
diffusion approximation), and (ii) the parameter error values are estimated separately from the parameter value itself and used to damp and regularize the inversions. The method of the AEKF algorithm is given by the following pseudocode[104]:

$$
\begin{aligned}
& \text { iterate } \\
& 1: \mathrm{x}=\mathrm{f}(\mathrm{y}) \\
& \text { for } i=1 \text { to \# source illuminations } \\
& \mathbf{J}_{i}=\frac{\partial \mathbf{x}_{i}}{\partial \mathbf{y}} \\
& \text { 3: } \mathbf{K}_{i}=\mathbf{P} \cdot \mathbf{J}_{i}{ }^{\mathbf{T}} \cdot\left(\mathbf{R}_{i}+\mathbf{Q}_{i}+\mathbf{J}_{i} \cdot \mathbf{P} \cdot \mathbf{J}_{i}{ }^{\mathbf{T}}\right)^{-1} \\
& \text { 4: } \Delta \mathbf{y}_{i}=\mathbf{K}_{i} \cdot\left(\mathbf{z}_{i}-\mathbf{x}_{i}\right) \\
& \text { 5: } \mathbf{P} \leftarrow \mathbf{P}-\mathbf{K}_{i} \cdot \mathbf{J}_{i} \cdot \mathbf{P} \\
& \text { end } \\
& \text { 6: } \mathbf{y} \leftarrow \mathbf{y}+\sum_{i} \Delta \mathbf{y}_{i} \\
& \text { until convergence }
\end{aligned}
$$

where the definitions of each term is given in Table 7.1.

Table 7.1. Definitions of terms used in AEKF pseudocode.

\begin{tabular}{|l|l|}
\hline $\mathbf{X}$ & distribution of predicted measurements \\
\hline $\mathbf{z}$ & corresponding experimental measurements \\
\hline $\mathbf{y}$ & unknown parameter (updated recursively) \\
\hline $\mathbf{J}$ & Jacobian matrix \\
\hline $\mathbf{K}$ & gain matrix \\
\hline $\mathbf{Q}$ & model error covariance \\
\hline $\mathbf{R}$ & measurement error covariance \\
\hline $\mathbf{P}$ & parameter error covariance \\
\hline $\mathbf{f}$ & forward solver \\
\hline
\end{tabular}

The parameter $\mathrm{x}$ represents the distributed predictions of the referenced measurements (the logarithm of AC and the phase shift for the case of frequency-domain measurements), which 
are generated by the forward simulator $f$. The parameter y represents the vector of current estimates of the unknown optical property, which is an inverse pseudo- $\square$ transform of the optical absorption coefficient $\mu_{\text {axi }}$ (in the case of fluorescence imaging, it is the absorption coefficient due to the fluorophore, $\mu_{\text {axf }}$ ). The measurement error covariance was assumed uncorrelated, and repeated observations (in this case, five) at each detector location were used to experimentally determine the variance for each source-detector pair. The model error covariance $\mathrm{Q}$ is difficult to estimate for unknown domains, and was empirically chosen to be one-fourth the mean of the measurement error variance. The discrepancies between measurements and predictions $(\mathrm{z}-\mathrm{x})$ are weighted by a gain matrix $\mathrm{K}$ in order to update the uncertain parameters $\mathrm{y}$ and the parameter error covariance $\mathrm{P}$. The gain matrix $\mathrm{K}$ is used to update the unknown optical parameter y and the parameter error covariance P. An initial variance estimate was set (typically 0.01 , however 0.1 was used for some in vitro cases due to inhomogeneous background) and the parameter error was assumed uncorrelated with the initial estimate.

A flow diagram of the AEKF based reconstruction algorithm used herein is illustrated in Figure 7.2. In fluorescence studies the reconstructed parameter is the absorption coefficient at the excitation wavelength $\left(\mu_{\text {axf }}\right)$. The algorithm starts with an initial guess of uniform distribution (herein, arbitrarily set to $0.003 \mathrm{~cm}^{-1}$ ), and uses the experimental data from the hand-held probe along with simulated measurements using the forward model (diffusion approximation) to get an updated distribution of the parameter of interest $\left(\mu_{\text {axf }}\right)$. Each updated distribution is then used in the algorithm until the convergence criteria is met and the reconstruction is complete. The AEKF algorithm is carried out recursively, i.e each set of measurements is used to continuously update the unknown parameter $y$ and the 
parameter error covariance $\mathrm{P}$, without storing the set of measurements from each loop. This recursive AEKF algorithm is carried out iteratively until the reconstructions converge. The convergence criteria was met when the root mean square output error RMSE was less than $1 \%$, or the total number of iterations exceeded 50 .

The AEKF reconstruction algorithm was developed for frequency-domain based fluorescence tomography. Modifications were made to the algorithm during the various tomography studies presented herein to adapt for continuous-wave based measurements, multiple-scan studies, and in vivo studies. The modification details will be given in the materials and methods for each tomography study appropriately.

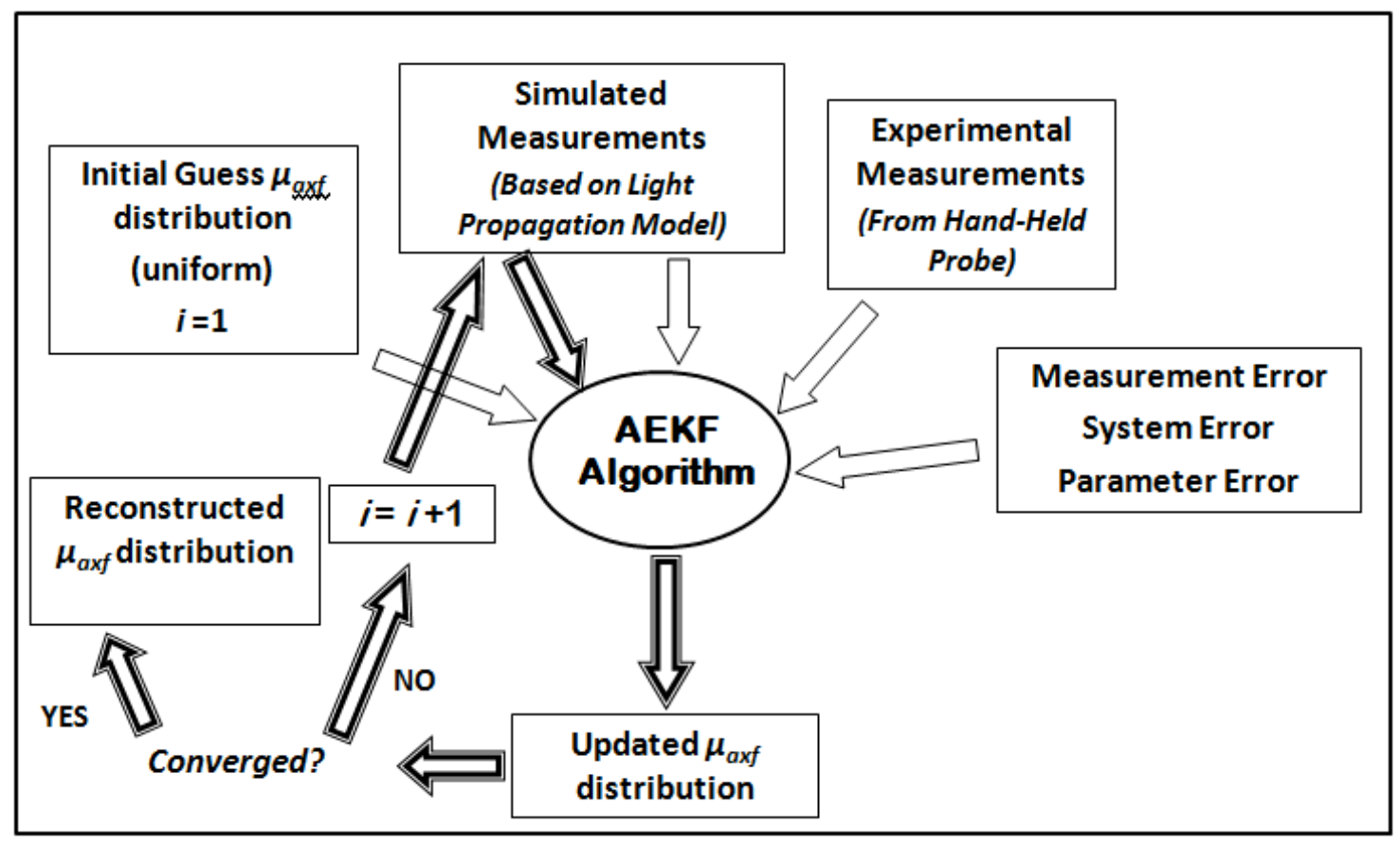

Figure 7.2 Flow diagram of the AEKF based reconstruction algorithm. 


\subsection{CONCLUSION}

This chapter detailed the reconstruction approach used in the tomography studies performed herein. The diffusion approximation of the radiative transport equation is used to model light propagation in biological tissue (forward problem). An approximate extended Kalman filter (AEKF) based reconstruction algorithm is used to reconstruct the unknown optical property distribution within tissue given experimental measurements at the tissue surface (inverse problem). The next chapters detail the experimental studies performed toward the goals of fast 2D imaging, coregistration, and 3D tomography in tissue phantoms, in vitro, and in vivo with human subjects. 


\section{CHAPTER 8}

\section{Study I: 2D Imaging in Tissue Phantoms and In Vitro in Near-Real Time}

\subsection{INTRODUCTION}

Real-time imaging is a useful tool for on-site cancer diagnosis in the clinical setting. Prior to performing 3D tomography studies using our hand-held optical imager, studies were performed to determine the ability of the device to detect a target via $2 \mathrm{D}$ imaging in near real-time. This chapter describes experimental studies that were performed in tissue phantoms and in vitro designed to detect the presence and estimate the 2D location of a fluorescent target using continuous wave based imaging.

\subsection{MATERIALS AND METHODS}

Fast 2D imaging was facilitated by the use of simultaneous multiple-point illumination and collection and CW based measurements. Studies were performed using slab tissue phantoms composed of $10 \times 10 \times 10 \mathrm{~cm}^{3}$ acrylic cubes filled with $650 \mathrm{ml}$ of $1 \%$ Liposyn solution (Liposyn II, 20\%, Henry Schein, Melville, NJ) in order to mimic the optical properties of a typical breast tissue. The fluorescent target was placed at different depths $(1.5-2.5 \mathrm{~cm})$ from the imaging surface and real-time as well as fast (subtracted) images of fluorescence intensity were acquired. The different experimental cases are summarized in Table 8.1.

Prior to in vivo studies with human subjects, experiments were performed using in vitro phantoms, which were composed of minced chicken breast combined with $1 \%$ Liposyn solution, in order to introduce a non-uniform scattering background. The in vitro mixture of minced chicken breast $(480 \mathrm{ml})$ and $1 \%$ Liposyn $(260 \mathrm{ml})$ was placed inside a $10 \times 10 \times 10 \mathrm{~cm}^{3}$ acrylic cube. Real-time as well as fast (subtracted) images of fluorescence intensity were 
acquired under different experimental conditions using either a $0.23 \mathrm{cc}$ or $0.45 \mathrm{cc}$ fluorescent target located at various depths between 1-2 cm (see Table 8.1).

Table 8.1 Summary of experimental cases for slab tissue phantom and in vitro studies.

\begin{tabular}{|c|c|c|c|c|c|}
\hline Subject Studied & Exp. \# & $\begin{array}{c}\text { Number of } \\
\text { Targets }\end{array}$ & $\begin{array}{c}\text { Target } \\
\text { Depth }(\mathrm{cm})\end{array}$ & $\begin{array}{c}\text { Target } \\
\text { Volume (cc) }\end{array}$ & $\begin{array}{c}\text { T:B } \\
\text { Contrast } \\
\text { Ratio }\end{array}$ \\
\hline \multirow{3}{*}{$\begin{array}{c}\text { Slab Tissue Phantom } \\
\text { (uniform scattering in } \\
\text { background) }\end{array}$} & 1 & 1 & 1.5 & 0.45 & $1: 0$ \\
\hline & 2 & 1 & 2.0 & 0.45 & $1: 0$ \\
\hline & 3 & 1 & 2.5 & 0.45 & $1: 0$ \\
\hline \multirow{6}{*}{$\begin{array}{l}\text { in vitro phantom (non- } \\
\text { uniform scattering in } \\
\text { background) }\end{array}$} & 4 & 1 & 1.0 & 0.45 & $1: 0$ \\
\hline & 5 & 1 & 1.5 & 0.45 & $1: 0$ \\
\hline & 6 & 1 & 2.0 & 0.45 & 1:0 \\
\hline & 7 & 1 & 1.0 & 0.23 & 1:0 \\
\hline & 8 & 1 & 1.5 & 0.23 & 1:0 \\
\hline & 9 & 1 & 2.0 & 0.23 & 1:0 \\
\hline
\end{tabular}

\subsection{RESULTS}

\subsubsection{Tissue Phantom Studies}

Real-time images using the slab phantom with uniform scattering in the background are shown in Figure 8.1 as $2 \mathrm{D}$ surface contour plots of the fluorescence intensity data with a target placed at different depths $(1.5-2.5 \mathrm{~cm})$ from the imaging surface. The non-uniform intensity distribution in Figure 8.1 is possibly due to the residual excitation leakage around 
the six source fibers (after the implementation of the subtraction technique). Additionally, the input laser source signal is not evenly distributed amongst the six source fibers, possibly causing a variation or non-uniformity in the output fluorescence intensity distribution.

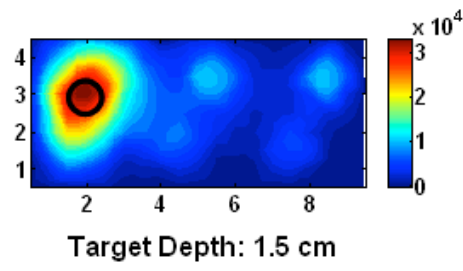

(A)

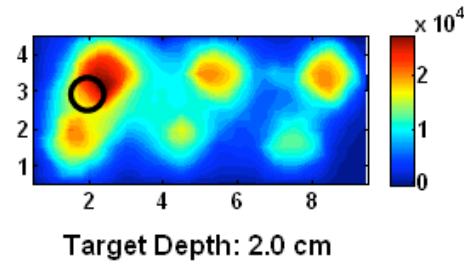

(B)

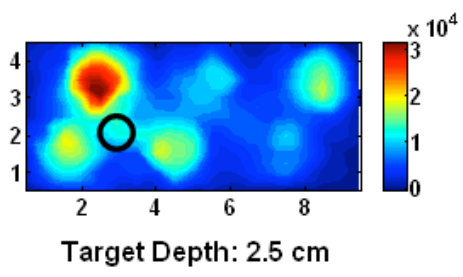

(C)

Figure 8.1 Near real-time images of fluorescence intensity obtained as 2D surface contour plots, acquired from slab phantoms (with uniform background scattering). The fluorescent target was placed at different locations and depths: (A) target location: $(\mathrm{x}, \mathrm{y}, \mathrm{z})=(2.0,2.7 .1 .5)$, and $(\mathrm{B})$ target location $(\mathrm{x}, \mathrm{y}, \mathrm{z})=(2.0,2.7,2.0)$ and $(\mathrm{C})$ target location: $(\mathrm{x}, \mathrm{y}, \mathrm{z})=(3.0,2.2,2.5)$. The black hollow circle in each sub-plot is the true target location.

The images show the feasibility of performing (close to) real-time $2 \mathrm{D}$ imaging using the hand-held device in tissue phantoms. The actual target location in the images is indicated in the figures by the black open circle in the $x-y$ plane for different target depths in the ' $\mathrm{z}$ ' direction. The fast $2 \mathrm{D}$ image estimates the $2 \mathrm{D}$ target location (instantly) in the $\mathrm{x}-\mathrm{y}$ plane. This information can then be further used towards 3D tomography (in the future) in order to determine the tumor volume, location, and depth [3].

From these plots, it is obvious that the real-time images are capable of differentiating the target from the background when the $0.45 \mathrm{cc}$ target was $1.5 \mathrm{~cm}$ deep. At greater tissue depths, the target was not distinctly differentiable due to the strong excitation leakage from the background. Upon applying the subtraction technique (as described in Section 6.4), the target is clearly differentiable from the background in all the experimental cases (see Figure 8.2). These subtracted images also have potential to obtain $3 \mathrm{D}$ target localization via 
tomographic imaging, as long as the probe's location on the tissue surface is coregistered with respect to the surface fluorescence images.

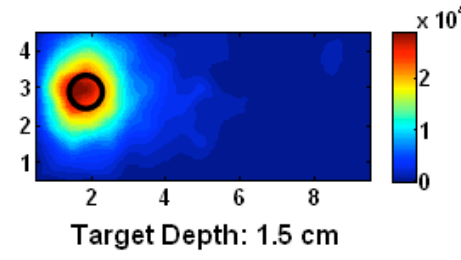

(A)

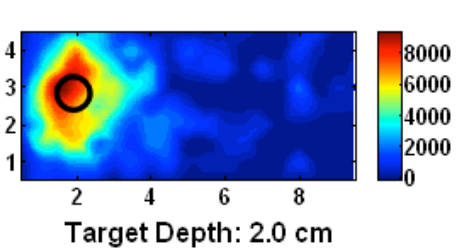

(B)

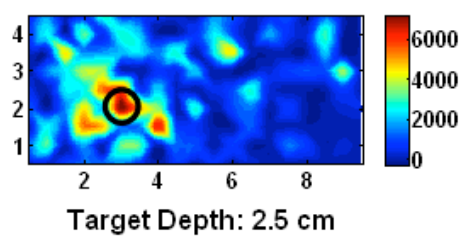

(C)

Figure 8.2 Fast subtracted images of fluorescence intensity obtained as $2 \mathrm{D}$ surface contour plots, acquired from slab phantoms (with uniform background scattering). The $0.45 \mathrm{cc}$ fluorescent target was placed at different locations and depths: $(A)$ target location: $(\mathrm{x}, \mathrm{y}, \mathrm{z})=(2.0,2.7 .1 .5)$, and (B) target location $(\mathrm{x}, \mathrm{y}, \mathrm{z})=(2.0,2.7,2.0)$ and $(\mathrm{C})$ target location: $(\mathrm{x}, \mathrm{y}, \mathrm{z})=(3.0,2.2,2.5)$. The black hollow circle in each sub-plot is the true target location.

\subsubsection{In Vitro Studies}

The results for the in vitro phantom experiments are shown in Figures 8.3 (real-time images) and 8.4 (fast subtracted images) for different target depths $(1-2 \mathrm{~cm})$ under T:B contrast ratio of 1:0. The true target location in the images is given as $x, y, z$ coordinates where ' $x$ ' is the lateral position, ' $\mathrm{y}$ ' is the height and ' $\mathrm{z}$ ' is the depth (distance from the imaging surface) which vary among the images. Due to heterogeneous scattering of the background phantom, only the subtracted images were capable of clearly differentiating the target from the background for targets deeper than $1.0 \mathrm{~cm}$. These studies show the ability of the handheld device to perform fast 2D surface imaging and target localization within a non-uniform scattering tissue-mimicking background.

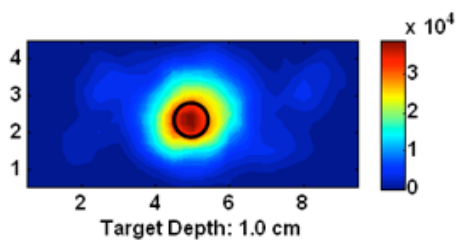

(A)

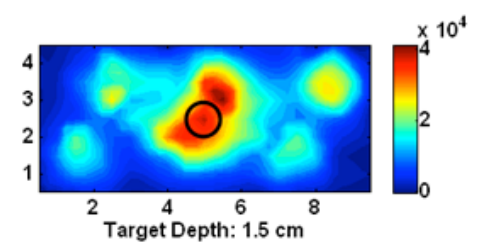

(B) 
Figure 8.3 Near-real time images of fluorescence intensity obtained as 2D surface contour plots, acquired from in-vitro slab phantoms (with non-uniform background scattering). The $0.45 \mathrm{cc}$ fluorescent target was located at a depth of (A) $1.0 \mathrm{~cm}$, and (B) $1.5 \mathrm{~cm}$, from the imaging surface. The black hollow circle in each sub-plot is the true target location.

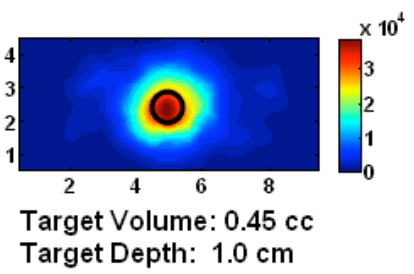

(A)

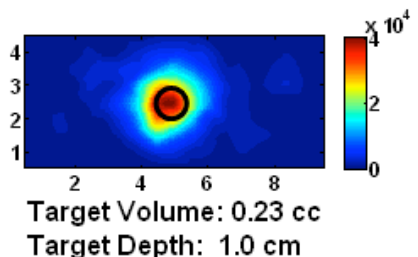

(D)

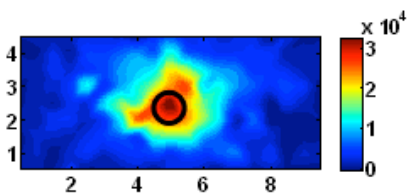

Target Volume: $0.45 \mathrm{cc}$

Target Depth: $1.5 \mathrm{~cm}$

(B)

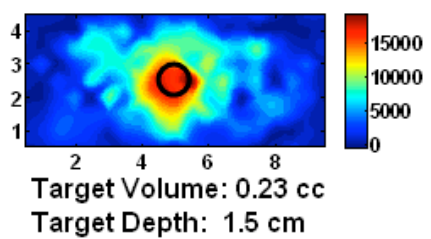

(E)

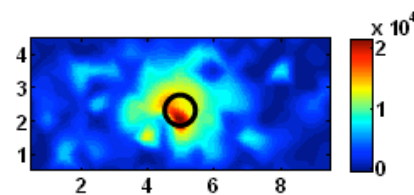

Target Volume: $0.45 \mathrm{cc}$

Target Depth: $2.0 \mathrm{~cm}$

(C)

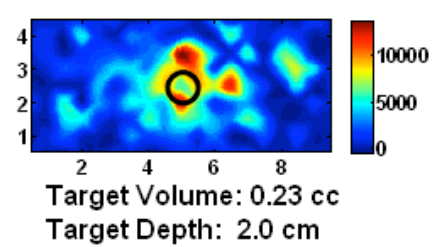

(F)

Figure 8.4 Fast subtracted images of fluorescence intensity obtained as $2 \mathrm{D}$ surface contour plots, acquired from in-vitro slab phantoms (with non-uniform background scattering). Images were collected for different target sizes and depths. Images (A)-(C) contain a target size of $0.45 \mathrm{cc}$ at depths of 1.0, 1.5, and $2.0 \mathrm{~cm}$ respectively. Images (D)-(F) contain a target size of $0.23 \mathrm{cc}$ at depths of 1.0, 1.5, and $2.0 \mathrm{~cm}$ respectively. The black hollow circle in each sub-plot is the true target location.

\subsection{DISCUSSION}

The fluorescence imaging studies described here demonstrate for the first time the acquisition of fast 2D surface images (in $<5 \mathrm{sec}$ ) of a fluorescent target in uniform tissue phantoms and in vitro using a hand-held based optical imaging device. The subtracted images have a potential to clearly differentiate target(s) from the background (under various experimental conditions), demonstrating the potential to translate the technology towards on-site breast imaging in a clinical environment. Additional experiments were performed with the target located at greater depths in the tissue phantoms, but the target was not detected at a depth of $2.5 \mathrm{~cm}$. At $2.5 \mathrm{~cm}$ deep, the detected signal from the target is close to 
the noise floor, and hence not differentiable from the background. A multi-location scanning approach is currently developed for differentiating deeply located or small volume targets from homogenous or heterogeneous background (details in Chapter 9). In short, this approach will incorporate the use of coregistered images obtained at multiple locations on the tissue surface, such that the targets can be differentiated from artifacts ${ }^{22}$ as well as the background [80]. When comparing Figures 8.2 and 8.4, it can be seen that the images from the in vitro phantom contain more noise than those from the uniform tissue phantom. This can possibly be attributed to the heterogeneous distribution of scattering properties or shifting of the chicken breast as the target is removed (which can cause a change in the signal distribution when the background image is collected). Experiments were also performed using the in vitro phantoms with a T:B contrast ratio of 100:1. However, the noise from the background signal dominated the image and a target was not detected even after subtracting the excitation background signal. Upon applying our multi-location scanning approach, the targets were differentiable under imperfect uptake conditions (i.e. T:B = 100:1). In addition to fast $2 \mathrm{D}$ imaging, the hand-held device described here has demonstrated $3 \mathrm{D}$ tomography of fluorescent targets with tissue phantoms using frequency-domain based measurements in order to estimate the 3D location and volume of the target within the tissue. [3]. Additional efforts involve the implementation of fast and automated coregistration facilities in order to enable precise 2D target localization (instantaneously) as well as 3D tomography studies in vitro as well as in vivo (Chapter 10 and 13, respectively).

\footnotetext{
22 Any signal that occurs in the image which is not from the target or tumor.
} 


\subsection{SUMMARY AND CONCLUSION}

Preclinical testing of a hand-held optical imager was performed to demonstrate the ability of the device to perform fast 2D imaging toward tumor detection in near real-time. Experimental studies show the ability of the device to detect and estimate the $2 \mathrm{D}$ location of a $0.45 \mathrm{~cm}^{3}$ fluorescent target up to $2.5 \mathrm{~cm}$ deep in a tissue phantom with homogeneous background and fluorescent targets of 0.23 or $0.45 \mathrm{~cm}^{3}$ volume up to $2.0 \mathrm{~cm}$ deep in vitro with heterogeneous background (more like a true tissue case). The goal of these studies was to determine if it was possible to detect a signal from the target using only fast 2D imaging. Target localization can be performed subsequently using 2D or 3D tomography. The results

presented herein demonstrate the potential of the device toward target detection and estimation of $2 \mathrm{D}$ target location even prior to $3 \mathrm{D}$ tomographic analysis. 


\section{CHAPTER 9}

\section{Study II: Coregistered Imaging towards Improved Target Depth Detection (using Multiple-scan Coregistered Imaging)}

\subsection{INTRODUCTION}

The feasibility of 2D target detection and 3D tomographic analysis using this hand-held optical imager has been demonstrated from tissue phantom and in vitro fluorescence imaging studies. During these studies, a single scan in the region of interest was acquired to detect and recover the embedded target(s) tomographically (using manually coregistered optical

images). However, it was observed that as the target depth was increased and/or its volume decreased, the target was not detectable from 2D images, nor was it recovered from 3D tomography studies. Experimental studies using single fast $2 \mathrm{D}$ images showed the ability to detect a target $\left(0.45 \mathrm{~cm}^{3}\right)$ up to $2.5 \mathrm{~cm}$ deep in tissue phantoms and up to $2.0 \mathrm{~cm}$ deep in vitro (details in section 8.3). Herein, an alternate imaging approach of using multiple-scans from various locations of the tissue surface is developed (using automatically coregistered optical images) and its feasibility to detect deeper targets is assessed [155].

In the current work, the recently developed multi-scan imaging approach is extensively assessed to determine the target detection limits of our hand-held optical imager. Studies were performed using the liquid tissue phantoms in order to determine the extent of improvement upon the previous studies using single-scan. Studies were also extended to in vitro tissue models in order to assess the performance of the device in a non-uniform (or heterogeneous) scattering background, in order to better mimic human tissue. 


\subsection{MATERIALS AND METHODS}

\subsubsection{Phantom and In Vitro Studies}

Experimental studies were performed initially using the simple case of the uniform liquid tissue phantom and were then extended to more realistic in vitro models. Phantom experiments were performed using tissue phantoms composed of 1\% Liposyn (800 mL) in a $10 \times 10 \times 10 \mathrm{~cm}^{3}$ acrylic cube. Acrylic sphere targets of sizes $0.23-0.45 \mathrm{~cm}^{3}$ filled with $1 \mu \mathrm{M}$ indocyanine green (ICG) were placed at different depths $(2.5-4.0 \mathrm{~cm})$ from the imaging surface to represent a tumor. Experiments were performed increasing the depth by $0.5 \mathrm{~cm}$ until the target was no longer detected. The experimental set-up is shown in Figure 9.1. The probe was placed in full contact with the phantom surface and multiple scans (2D coregistered images) were collected using the method described in section 2.5 below.
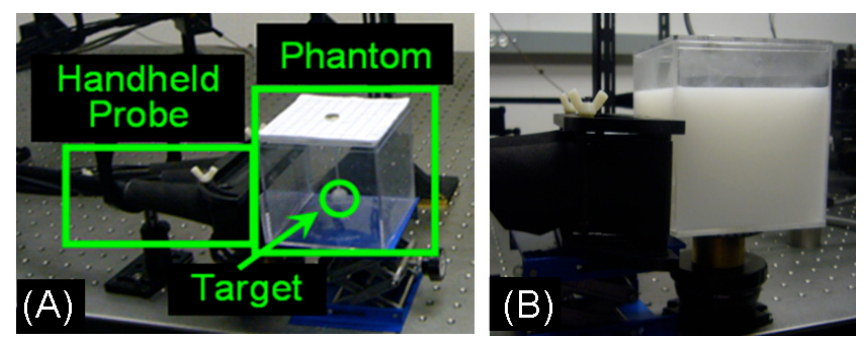

Figure 9.1 Experimental set-up for phantom studies. (A) A spherical target filled with $1 \mu \mathrm{M}$ indocyanine green is enclosed within the cubical phantom to represent a tumor. (B) The phantom is composed of a $1 \%$ Liposyn solution to mimic the optical properties of human breast tissue.

To represent the heterogeneous nature of human tissue, studies were also performed in vitro using minced chicken breast $(480 \mathrm{~mL})$ combined with 1\% Liposyn $(260 \mathrm{~mL})$ in a $10 \times 10 \times 10 \mathrm{~cm}^{3}$ acrylic cube in order to introduce a background of non-uniform scattering. The $0.45 \mathrm{~cm}^{3}$ fluorescent target was placed at different depths between $2.0-4.0 \mathrm{~cm}$, until the target was no longer detected. The experiments were performed for the perfect uptake case 
with tumor-to-background ratio (T:B) of 1:0 and imperfect uptake case with T:B of 100:1. Table 9.1 summarizes the different experimental studies performed in which a target was detected. For each image collected, a subtraction-based post-processing technique was used to eliminate excitation light leakage (as described in Section 6.4).

Table 9.1 Summary of experimental studies in which a target was detected in tissue phantoms and in vitro. The cases where the deepest target was detected for phantom or in vitro, and perfect or imperfect uptake are highlighted in red.

\begin{tabular}{|c|c|c|c|c|}
\hline $\begin{array}{l}\text { Experimental } \\
\text { Case }\end{array}$ & $\begin{array}{l}\text { Experiment } \\
\#\end{array}$ & $\begin{array}{l}\text { Target } \\
\text { Volume } \\
(\mathrm{cm} 3)\end{array}$ & $\begin{array}{l}\text { Target } \\
\text { Depth } \\
(\mathrm{cm})\end{array}$ & T:B \\
\hline \multirow{10}{*}{$\begin{array}{l}\text { Tissue } \\
\text { Phantom }\end{array}$} & 1 & 0.45 & 3.0 & $1: 0$ \\
\hline & 2 & 0.45 & 3.5 & $1: 0$ \\
\hline & 3 & 0.23 & 2.5 & $1: 0$ \\
\hline & 4 & 0.23 & 3.0 & $1: 0$ \\
\hline & 5 & 0.23 & 3.5 & 1:0 \\
\hline & 6 & 0.45 & 2.5 & $100: 1$ \\
\hline & 7 & 0.45 & 3.0 & 100:1 \\
\hline & 8 & 0.23 & 2.0 & 100:1 \\
\hline & 9 & 0.23 & 2.5 & 100:1 \\
\hline & 10 & 0.23 & 3.0 & 100:1 \\
\hline \multirow{5}{*}{ In Vitro } & 11 & 0.45 & 2.5 & $1: 0$ \\
\hline & 12 & 0.45 & 3.0 & $1: 0$ \\
\hline & 13 & 0.45 & 3.5 & 1:0 \\
\hline & 14 & 0.45 & 2.0 & 100:1 \\
\hline & 15 & 0.45 & 2.5 & 100:1 \\
\hline
\end{tabular}

\subsubsection{Validation of Coregistered Imaging in Phantoms}

In order to quantitatively determine the accuracy of the tracked position and coregistered image compared to the true location on the phantom, the probe was placed at five different reference points of known locations (moving $0.5 \mathrm{~cm}$ vertically) and five measured values were recorded from the tracking software at each position. The average, standard deviation, 
and discrepancy of these measured values for each position are given in Table 9.2. It can be seen from the standard deviation of the measured values that the tracked probe position fluctuates between $0.013-0.094 \mathrm{~cm}$. The average total distance off represents the accuracy of the tracked position compared to the true location. The average total distance off is $\sim 0.19$ cm. The deviation and discrepancy of the measured values can be attributed to instrumentation error such as fluctuation in the tracked position of the probe. Currently, efforts are made to overcome these limitations and improve the stability of the tracking system (Chapter 16).

Table 9.2 Average and standard deviation of x,y,z coordinates as displayed in tracking software and total distance off between true and measured values. Values are in units of centimeters and each measured value represents an average of 5 measurements while probe was at each of the 5 positions.

\begin{tabular}{|c|c|c|c|c|}
\hline & $\begin{array}{c}\text { True Value } \\
(x, y, z)\end{array}$ & $\begin{array}{l}\text { Average Measured } \\
\text { Value }(x, y, z)\end{array}$ & $\begin{array}{c}\text { Standard Dev. of } \\
\text { Measured Value }(x, y, z)\end{array}$ & $\begin{array}{c}\text { Total Distance Off } \\
(\mathrm{cm})\end{array}$ \\
\hline Position \#1 & $(0,0,0)$ & $(0.14,0.12,-0.28)$ & $(0.026,0.034,0.015)$ & 0.34 \\
\hline Position \#2 & $(0,0,0.5)$ & $(0.004,0.12,0.47)$ & $(0.094,0.044,0.019)$ & 0.15 \\
\hline Position \#3 & $(0,0,1)$ & $(-0.26,0.098,0.99)$ & $(0.013,0.019,0.018)$ & 0.28 \\
\hline Position \#4 & $(0,0,1.5)$ & $(0.08,0.036,1.50)$ & $(0.021,0.021,0.011)$ & 0.09 \\
\hline Position $\# 5$ & $(0,0,2)$ & $(0.002,0.088,2.00)$ & $(0.026,0.008,0.013)$ & 0.09 \\
\hline
\end{tabular}

\subsubsection{Multiple Scan Technique}

The multiple scan technique involves collecting a series of scans at different probe positions and coregistering each image to its appropriate location. A scan is defined as a $2 \mathrm{D}$ surface contour plot of fluorescence intensity data collected from a CW image (the mean of 5 repeated images at the same location) using the ICCD based detection system. Each image is immediately coregistered to the discretized phantom mesh. By scanning at multiple locations, the weak fluorescence signal from the target (which appears at the same location 
on the tissue geometry during each scan) can possibly dominate the strong excitation light leakage (which tends to appear at different locations on the tissue geometry during each scan).

Different combinations of image positions were used to determine the optimal multi-scan method. Initially 4-5 scans were collected moving the probe $0.5 \mathrm{~cm}$ in the vertical direction between each scan. For the same experimental case, 9 scans were collected moving the probe in $0.25 \mathrm{~cm}$ increments in the vertical direction. Additionally, repeated scans were collected at each probe location. It was determined that collecting 2-3 repeated scans (i.e. $2-3 \times 5$ repeated images or 10-15 repeated images) at each probe location for 4-5 positions $0.5 \mathrm{~cm}$ apart resulted in better target detectability with fewer artifacts than collecting single scans (i.e. $1 \times 5$ repeated images) at each probe location for 9 positions 0.25 cm apart. This can possibly be attributed to two reasons: (i) the inaccuracy (from instability) of the positional tracking system (described in section 2.3) has greater impact at smaller positional increments (e.g. $0.25 \mathrm{~cm}$ ), leading to increased artifacts; and (ii) the variability in the instrument's response is minimized by increasing the number of repeated scans (or images) at the same location. The number of repeated scans (or images) can be increased for the case where the probe locations were $0.25 \mathrm{~cm}$ apart. However, this further increases the overall imaging time and hence was not attempted. In the future, for an in vivo case, multiple images can be collected and summed from arbitrary probe positions and the number of scans can be optimized to minimize the overall imaging time and number of artifacts. The position will be known since the image is immediately coregistered at its location. 


\subsection{RESULTS}

Figure 9.2 shows four single images (2D contour plots of fluorescence intensity) for experimental case \# 12 where a $0.45 \mathrm{~cm}^{3}$ fluorescent target was placed $3.0 \mathrm{~cm}$ deep in vitro under perfect uptake condition $(\mathrm{T}: \mathrm{B}=1: 0)$. Multiple scans were collected as described in section 2.5. In each image, the true target location is indicated by a black open circle. The images show that the target is not detected in a single scan, and only random distributions of artifacts (high intensity signals that appear in the image which do not originate from the target) are visible.

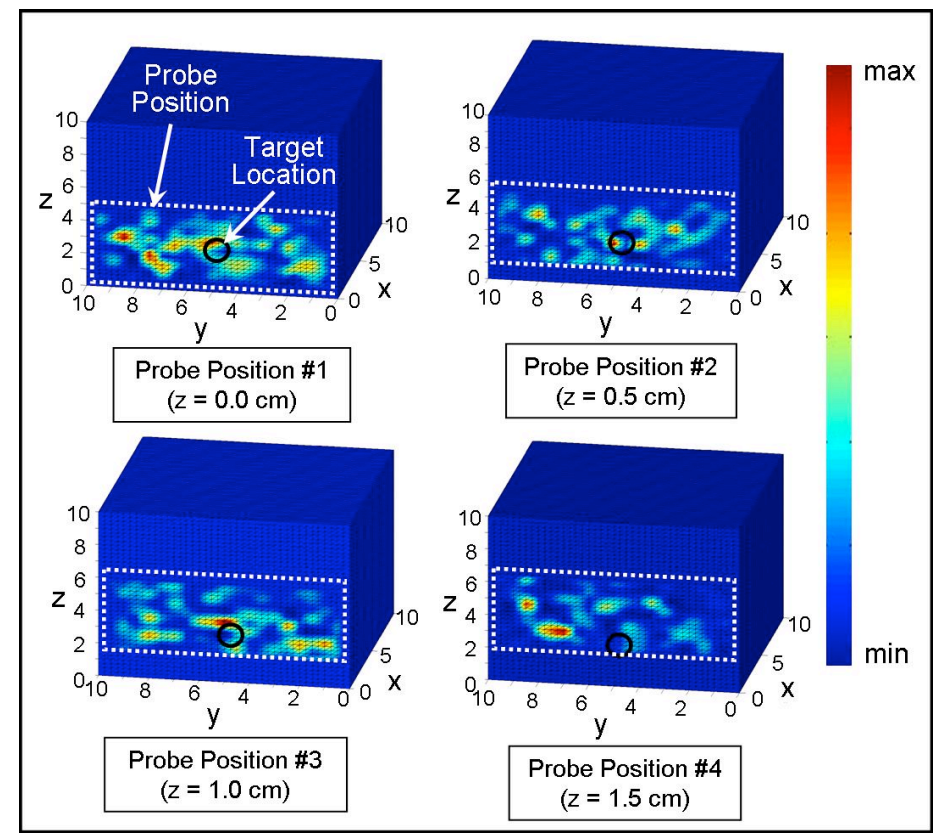

Figure 9.2 Coregistered images from single scans (2D contour plots of fluorescence intensity data) at four probe positions for experimental case $\# 12$ (a $0.45 \mathrm{~cm} 3$ fluorescent target placed $3.0 \mathrm{~cm}$ deep, $\mathrm{x}$-dimension in-vitro phantom under $\mathrm{T}: \mathrm{B}=1: 0)$. In each image, the white dotted line represents the probe position with respect to the phantom and the black open circle represents the true target location. 
Upon summation of eight scans, the fluorescent signal is detected at the target location (Figure 9.3). This can be attributed to the random distribution of artifacts that appear in different locations for each single scan, while the signal from the target remains in the same location relative to its coregistered position on the phantom. Upon summation of the coregistered images, the random signals from the artifacts tend to diminish compared to the consistent signal from the target, which tends to intensify.

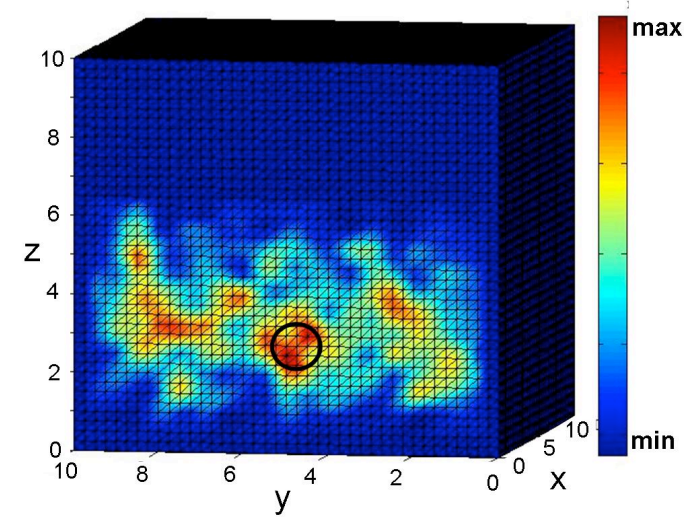

Figure 9.3 Summated image of multiple scans shown in Figure 9.2 (experimental case \#12). The summed image represents summation of 8 single scans, where 2 scans were collected at each of the 4 probe positions shown in Figure 9.3, The black open circle represents the true target location.

Figure 9.4 shows the result for summated multi-scan images for experimental cases 5, 10, 13, and 15 (Table 9.1, bold). Case 5 represents a $0.23 \mathrm{~cm}^{3}$ target placed $3.5 \mathrm{~cm}$ deep in the tissue phantom under perfect uptake conditions $(\mathrm{T}: \mathrm{B}=1: 0)$. The target was detected close to the true location and no artifacts were present. Case 10 represents a $0.23 \mathrm{~cm}^{3}$ target placed $3.0 \mathrm{~cm}$ deep in the tissue phantom under imperfect uptake condition ( $T: B=100: 1)$. The target was detected at the true location with some diffused signal around it. Case 13 represents a $0.45 \mathrm{~cm}^{3}$ target placed $3.5 \mathrm{~cm}$ deep in vitro under perfect uptake conditions $(\mathrm{T}: \mathrm{B}=1: 0)$. The target was detected at the true location with artifacts also visible far from 
the target location. These artifacts can be attributable to the heterogeneous nature of the in vitro phantom.
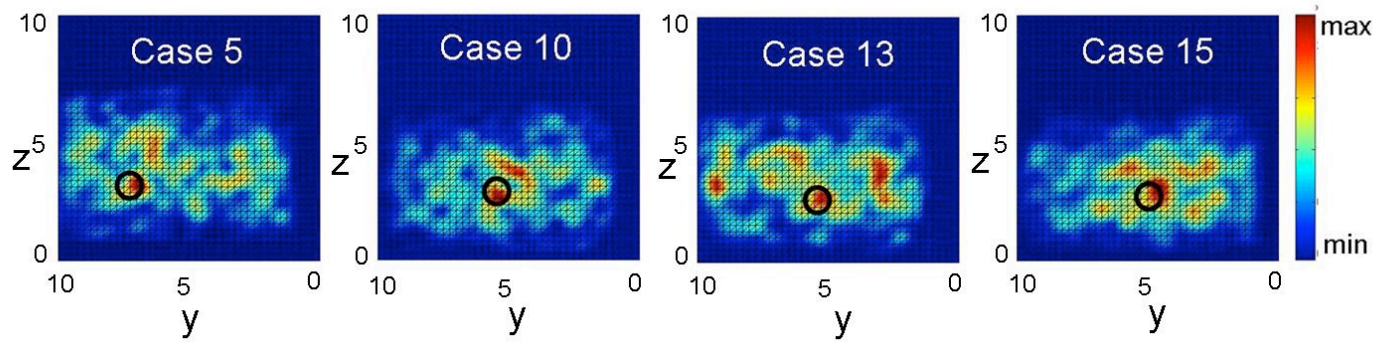

Figure 9.4 Summed images of multiple coregistered scans from the four best experimental cases listed in Table 1. The black open circle indicates the true target location for each case.

Case 15 represents a $0.45 \mathrm{~cm} 3$ target placed $2.5 \mathrm{~cm}$ deep in-vitro under imperfect uptake conditions $(\mathrm{T}: \mathrm{B}=100: 1)$. The target was detected close to the true location along with minimal artifacts around it.

The initial experiments designed to detect deeper targets consisted of performing a single scan at $4-5$ positions moving the probe $0.5 \mathrm{~cm}$ in the vertical (z) direction. For deeper targets $(>3.0 \mathrm{~cm})$, heterogeneous (in vitro) phantoms, and/or imperfect uptake cases, the target was not detected upon summation of four or five scans. However, it was observed that when multiple repeated scans were collected at the same location for each probe position, the targets were detectable. In other words, increasing the number of repeated scans is as important as increasing the number of scan locations towards deep target detection under heterogeneous and/or imperfect uptake conditions.

The results presented here demonstrate that deeper and smaller targets can be detected using the multiple-scan approach than using a single-scan alone. The impact of these results extend beyond target detection in $2 \mathrm{D}$ in that the information can be used as a priori 
information to aid 3D reconstruction using a single frequency-domain image (or scan) towards deeper target recovery (future work).

\subsection{DISCUSSION}

The prime hypothesis for the current study is that a single scan (of a large area, $4 \mathrm{x} 9 \mathrm{~cm}^{2}$ ) can fail in detecting deeper targets during a near real-time optical imaging study. Upon scanning at multiple locations on the surface of a tissue geometry, the possibility to detect deeper targets and differentiating them from artifacts tends to improve upon summing all these multiple scans. The detection limits of our hand-held optical imager, the first of its kind that can scan large areas in near real-time, has been $2.5 \mathrm{~cm}$ for a $0.45 \mathrm{~cm}^{3}$ target under $\mathrm{T}: \mathrm{B}=1: 0$ from the past studies (Chapter 8 ) involving a single scan from the region of interest on the tissue geometry. In the current study, it was clearly observed that upon summation of the intensity signals from multiple scans of the tissue surface, targets of smaller volume and as deep as $3.5 \mathrm{~cm}$ were detectable. However, artifacts start appearing when the target depth is increased, the target volume is decreased, and/or heterogeneity of the background increased. The reasons for these artifacts are two-fold: (i) The uneven source strength distribution of the 6 simultaneous sources affects the ability to detect the target depending on the proximity of the target to the stronger sources. As multiple scans are collected the strong source may move away from the target resulting in a more diffused signal which produces a greater number of artifacts upon subtraction of the background. (ii) The positional information of the hand-held probe is not accurately coregistered due to the instability of the motion tracking device, which can lead to misalignment of the target signal in the multiple images. As a result, some signals which originated from the target can be mistaken as artifacts due to their improper location in the image. Currently, work is carried 
out to homogenize the source strength distribution by altering the instrumentation set-up at the source end, and also developing alternate motion tracking approaches that are stable and more accurate in comparison to the current device.

Researchers in the past have demonstrated detection of targets as deep as $5 \mathrm{~cm}$ using their hand-held optical devices [50]. However, the detection was typically a point location that was spectroscopically obtained from the tissue surface. In addition, the specificity of getting a negative result (not detecting any target) in the absence of the target (i.e. in the surrounding tissues) is not described. In other words, unlike the current hand-held optical imager that can produce 2D images over large areas in near real-time, the other hand-held imagers have a limited imaging area (mostly point-based imaging) and typically produce only spectroscopic measurements from a few sources/detectors.

The advantage of the current hand-held imager's optical data is that it can be applied towards 3D tomography studies, since the positional location of the optical data with respect to the 3D tissue geometry is coregistered during imaging studies. Hence, the implementation of the multi-scan imaging approach and using the summated images towards immediate 2D deep target detection and future 3D tomographic analysis is feasible. Although the present study is focused on fluorescence-enhanced optical imaging, the multiscan summation and imaging approach is applicable for absorption-based diffuse optical imaging studies as well.

In the area of fluorescence tomographic imaging, Sevick's research group was one of the first groups to demonstrate 3D fluorescence optical tomography in clinically relevant sized tissue phantoms, using large bulky optical imaging instrumentation [24]. Fluorescent targets of $1 \mathrm{~cm}^{3}$ volume were recovered at a depth up to $2.8 \mathrm{~cm}$ under perfect uptake 
conditions. The first in vivo 3D fluorescence optical tomography of breast cancer in human subjects was demonstrated by Corlu et. al. where tumors were recovered in vivo up to $2.0 \mathrm{~cm}$ deep in human breast tissue [110]. From our past 3D tomography studies using the handheld imager on large tissue phantoms, $0.45 \mathrm{~cm}^{3}$ targets as deep as $2.5 \mathrm{~cm}$ were recovered in uniform tissue phantoms under perfect uptake conditions [3].

In a recent in vivo study using a simulated fluorescent target, a single scan using our hand-held optical imager was able to detect a $0.23 \mathrm{~cm}^{3}$ target $\sim 2.5 \mathrm{~cm}$ deep in human breast tissue (Chapter 11). These studies demonstrate the potential that upon using multiple scans and applying the summation approach, deeper and smaller targets can become detectable from the tissue surface. In addition, these summated images can also assist in tomographically recovering deeper targets beyond what is demonstrated to date in the area of fluorescence optical tomography. However, the developed hand-held optical imager is limited to reflectance based imaging, which tends to limit the recovery of the target's true depth during 3D tomography. Currently, research is carried out to develop alternate imaging approaches using the hand-held device such that trans-illumination measurements can also be acquired in an attempt to improve the target depth recovery (during 3D tomography).

\subsection{CONCLUSION}

Herein we have demonstrated improved detection limits via application of a multiple-scan technique. The multi-scan imaging approach is facilitated by the use of fast 2D coregistered imaging in $\mathrm{CW}$ mode. Previous results using single-scan imaging showed that the greatest target depth detected was $2.5 \mathrm{~cm}$ and $1.5 \mathrm{~cm}$ for a $0.45 \mathrm{~cm}^{3}$ target in a (liquid) tissue phantom under perfect and imperfect uptake conditions, respectively. By using the multiscan technique, this depth was improved to $3.5 \mathrm{~cm}$ for a smaller target $\left(0.23 \mathrm{~cm}^{3}\right)$ under the 
$\mathrm{T}: \mathrm{B}=1: 0$ condition. Under imperfect uptake conditions $(\mathrm{T}: \mathrm{B}=100: 1)$, the detectable target depth was increased from $1.5 \mathrm{~cm}$ to $3.0 \mathrm{~cm}$. Since these results were promising, studies were extended to in vitro models, which better represent the non-uniformity of human tissue. From these in vitro studies the target was detected at depths of $3.5 \mathrm{~cm}$ and $2.5 \mathrm{~cm}$ for perfect (1:0) and imperfect uptake (100:1) cases, respectively. These studies demonstrate that summation of multiple coregistered images can be used towards deeper target detection than is capable from single scans alone. In a clinical setting, this technique can be used to acquire multiple images quickly in order to detect the presence of a tumor, determine its $2 \mathrm{D}$ location within the tissue, and also perform 3D tomography studies (as a follow-up). Thus, the handheld optical imager has potential for fast 2D imaging and 3D tomography in the clinical setting for breast cancer diagnosis. 


\section{CHAPTER 10}

\section{Study III: 3D Tomography Studies in Tissue Phantoms and In Vitro}

\subsection{INTRODUCTION}

Parallel to the coregistered imaging studies described in Chapter 9, 3D tomography studies were carried out in tissue phantoms and in vitro with simple geometries using manual coregisteration. This chapter describes FDPM tomography studies using a single image and $\mathrm{CW}$ tomography studies using multiple summated images towards recovery of targets at greater depths.

\subsection{MATERIALS AND METHODS}

\subsubsection{FDPM Based Reconstructions In Vitro}

Three-dimensional fluorescence tomography studies were performed from FDPM measurements collected using the hand-held device with in vitro phantoms. The phantoms were composed of minced chicken breast $(480 \mathrm{~mL})$ and 1\% Liposyn $(260 \mathrm{~mL})$ for a total

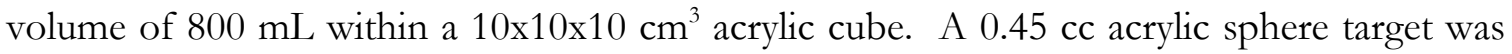
filled with $1 \mu \mathrm{M}$ ICG and placed at different locations and depths within the phantom. A FDPM image was collected with the target followed by a background image with the target removed to use to subtract the excitation signal. In all cases the laser power was set to 217 $\mathrm{mA}$, the frequency synthesizer was set to $2.7 \mathrm{~dB}$, and the exposure time was $200 \mathrm{msec}$. The gain setting on the image intensifier was adjusted for each data set and held constant between images with and without a target. The optical properties of the phantom $\left(\mu_{\mathrm{ax}}, \mu_{\mathrm{sx}}\right.$ ', $\mu_{\mathrm{am}}$, and $\mu_{\mathrm{sm}}$ ') were measured at both the excitation (' $\mathrm{x}$ ') and emission (' $\mathrm{m}$ ') wavelengths (785 and $830 \mathrm{~nm}$, respectively) using a single pixel homodyne FDPM optical property measurement system. Multiple repetitions (5-10) of the optical property measurements were 
collected and averaged for each in vitro phantom. The phantom was stirred between each measurement in order to acquire values that are an average representation of the mixture as a whole. An example optical property measurement set including mean and standard deviation of the optical properties at each wavelength is shown in Table 10.1. The table shows large standard deviations due to the heterogeneous nature of the phantom material, and hence ten measurements were collected and averaged for each wavelength. The output intensity of the six source points at the probe face were measured using a digital optical power meter (PM121 w/ S121 sensor, 500nW-500mW, Thorlabs, NJ) and average values were used as input parameters in the reconstruction algorithm. The initial guess of the reconstructed parameter $\left(\mu_{\text {axf }}\right)$ was arbitrarily set to a uniform distribution of $0.003 \mathrm{~cm}^{-1}$ and an initial p-value of 0.01 was used in all cases. Table 10.2 provides a summary of the experiment parameters for various experimental cases including the average measured source intensities, the gain setting on the image intensifier, the actual target location in $\mathrm{cm}$, the actual target volume, the measured optical property values of the phantom at the excitation and emission wavelengths, the target to background ratio (T:B), the recovered target location in $\mathrm{cm}$, the recovered $\mu_{\text {axf }}$ values in $\mathrm{cm}^{-1}$, the recovered target volume, the number of iterations executed before convergence, and the breakpoint (cutoff value selected on the first breakpoint of a histogram plot of the recovered $\mu_{\text {axf }}$ values). The total distance off between the true and recovered target locations was calculated using the following equation:

$$
\text { dist. }=\sqrt{\left(x_{\text {res. }}-x_{\text {true }}\right)^{2}+\left(y_{\text {res. }}-y_{\text {true }}\right)^{2}+\left(z_{\text {rec }}-z_{\text {true }}\right)^{2}}
$$


Table 10.1 Sample optical property measurements of the in vitro phantom. All optical property values are in units of $\mathrm{cm}^{-1}$.

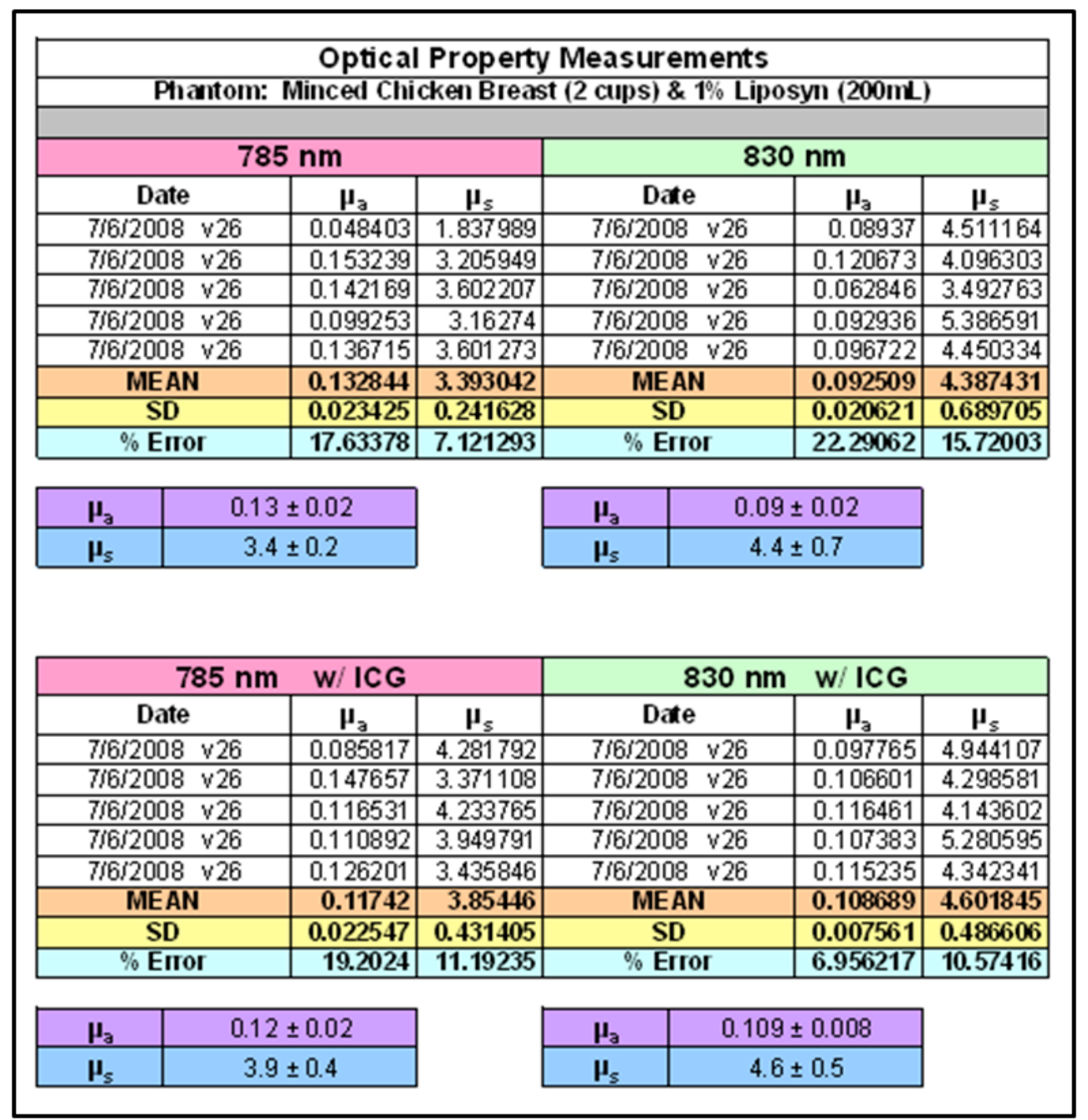

The results from two example cases (highlighted in yellow in Table 10.2) are given in Section 10.3.1.

\subsubsection{CW Based Reconstructions using Summed Multiple-scan Data}

Three-dimensional (3D) reconstructions were performed using the CW based data from all the multiple-scan experimental cases detailed in Chapter 9 (summarized in Table 9.1). For the $\mathrm{CW}$ based reconstructions, the modulation was set to zero in the reconstruction algorithm for continuous amplitude. Each experimental set encompassed a series of 8 scans, 
2 repetitions at 4 different probe locations moving vertically in $0.5 \mathrm{~cm}$ increments (see Section 9.2.2 for more details on the multiple-scan acquisition). The $2 \mathrm{D}$ images of the summed multiple-scan data were presented in Section 9.3. Herein, 3D reconstructions were performed using two methods: (i) individual reconstructions were performed using the data from each single scan and the recovered $\mu_{\text {axf }}$ values were then summed; (ii) the initial raw data from the 8 scans was summed together into a single data set which was then used to perform 3D reconstructions. A separate mesh was generated to input in the reconstruction algorithm for the different data sets, with different nodes assigned to the source and detector coordinates according to the coregistered location of the probe with respect to the phantom.

\subsection{RESULTS AND DISCUSSION}

\subsubsection{FDPM Based Reconstructions In Vitro}

Figure 10.1 shows the result for experimental case \#26 (Table 10.2) with a target located 2.0 $\mathrm{cm}$ deep from the imaging surface under perfect uptake conditions. The $2 \mathrm{D}$ contour plot represents the subtracted fluorescence intensity data collected in the frequency domain and the three isosurface contour plots show three different views of the $3 \mathrm{D}$ reconstruction of the target. These plots show a recovered target; however the absorption coefficient values of the fluorophores (labeled as V1 in Figure 10.1) are low (close to the initial distribution of 0.003). The total distance off between the true and recovered target location was $1.9 \mathrm{~cm}$. This large value is mostly attributable to the z-coordinate which represents the depth of the target from the imaging surface. 
Table 10.2 Summary of the experiment parameters for two sample experimental cases including the average measured source intensities, the gain setting on the image intensifier, the actual target location in $\mathrm{cm}$, the actual target volume, the measured optical property values of the phantom at the excitation and emission wavelengths, the target to background ratio (T:B), the recovered target location in $\mathrm{cm}$, the recovered $\mu_{\text {axf }}$ values in $\mathrm{cm}^{-1}$, the recovered target volume, the number of iterations executed before convergence, and the breakpoint (cutoff value selected on the first breakpoint of a histogram plot of the recovered $\mu_{\text {axf }}$ values). A full table of all experimental cases performed is given in Appendix C.

\begin{tabular}{|c|c|c|c|c|c|c|c|c|c|c|c|c|c|c|c|}
\hline $\begin{array}{c}\text { Expt. } \\
\#\end{array}$ & $\begin{array}{c}\text { Ave. Source } \\
\text { Int. } \\
\text { (s1, s2, s3, } \\
\text { S4, s5, s6) } \\
\text { (mW) }\end{array}$ & $\begin{array}{c}\text { Gai } \\
\mathrm{n}\end{array}$ & $\begin{array}{c}\text { Act. } \\
\text { Targ. } \\
\text { Location } \\
(x, y, z)\end{array}$ & \begin{tabular}{|c|} 
Actual \\
Target \\
Volum \\
$e$ \\
\end{tabular} & $\boldsymbol{\mu}_{\mathrm{ami}}$ & $\mu_{\text {axi }}$ & $\boldsymbol{\mu}_{\mathrm{sxi}}$ & $\boldsymbol{\mu}_{\mathrm{smi}}$ & $\mathrm{T}: \mathrm{B}$ & $\begin{array}{l}\text { Reconst. } \\
\text { Location } \\
(x, y, z)\end{array}$ & $\begin{array}{c}\text { Recover } \\
\text { ed } \mu_{\text {axf }}\end{array}$ & $\begin{array}{c}\text { Rec. } \\
\text { Targ. } \\
\text { Vol. } \\
\end{array}$ & $\begin{array}{c}\# \\
\text { Iter. } \\
\end{array}$ & $\begin{array}{c}\text { Break- } \\
\text { point }\end{array}$ & $\begin{array}{c}\text { Initial } \\
P \text { - } \\
\text { value }\end{array}$ \\
\hline 26 & $\begin{array}{l}0.614,0.580, \\
0.068,1.859, \\
0.044,1.408\end{array}$ & 5.4 & $\begin{array}{c}(4.0,2.5 \\
2.0)\end{array}$ & $0.45 \mathrm{cc}$ & 0.11 & 0.08 & 6.16 & 5.73 & 1:0 & $\begin{array}{c}(4.58,2.33 \\
0.20)\end{array}$ & $4.20 \mathrm{E}-05$ & 0.014 & 2 & 0.00301 & 0.01 \\
\hline 27 & $\begin{array}{l}0.614,0.580, \\
0.068,1.859, \\
0.044,1.408\end{array}$ & 5.4 & $\begin{array}{c}(4.0,2.5 \\
2.5)\end{array}$ & $0.45 \mathrm{cc}$ & 0.11 & 0.08 & 6.16 & 5.73 & 1:0 & $\begin{array}{c}(3.71,3.21, \\
0.70)\end{array}$ & $3.26 \mathrm{E}-04$ & 0.108 & 2 & 0.003 & 0.01 \\
\hline
\end{tabular}

The true $\mathrm{z}$-coordinate was $2 \mathrm{~cm}$, while the recovered $\mathrm{z}$-coordinate was only $0.2 \mathrm{~cm}$ (Table 10.2). The limited depth recovery is due to the physics of reflectance based measurements and has been reported by other research groups in the literature.[23,105,114]

Figure 10.2 shows the 3D reconstruction result for experimental case \#27 (Table 10.2) with a target located $2.5 \mathrm{~cm}$ deep under perfect uptake conditions. The $2 \mathrm{D}$ contour plot shows the presence of more artifacts at this deeper target location; however the isosurface plots of the $3 \mathrm{D}$ reconstructions show that the target was recovered at this depth. Similar to the previous result, the recovered absorption coefficient values of the fluorophores (labeled as V1 in Figure 10.2) are low (close to the initial distribution of 0.003), the reconstructed location was close to the true location in the $x-y$ plane, and the recovered depth is shallow compared to the true depth. The total distance off between the true and recovered target location was $2.0 \mathrm{~cm}$, which is mostly attributable to the depth ( $\mathrm{z}$ coordinate). 


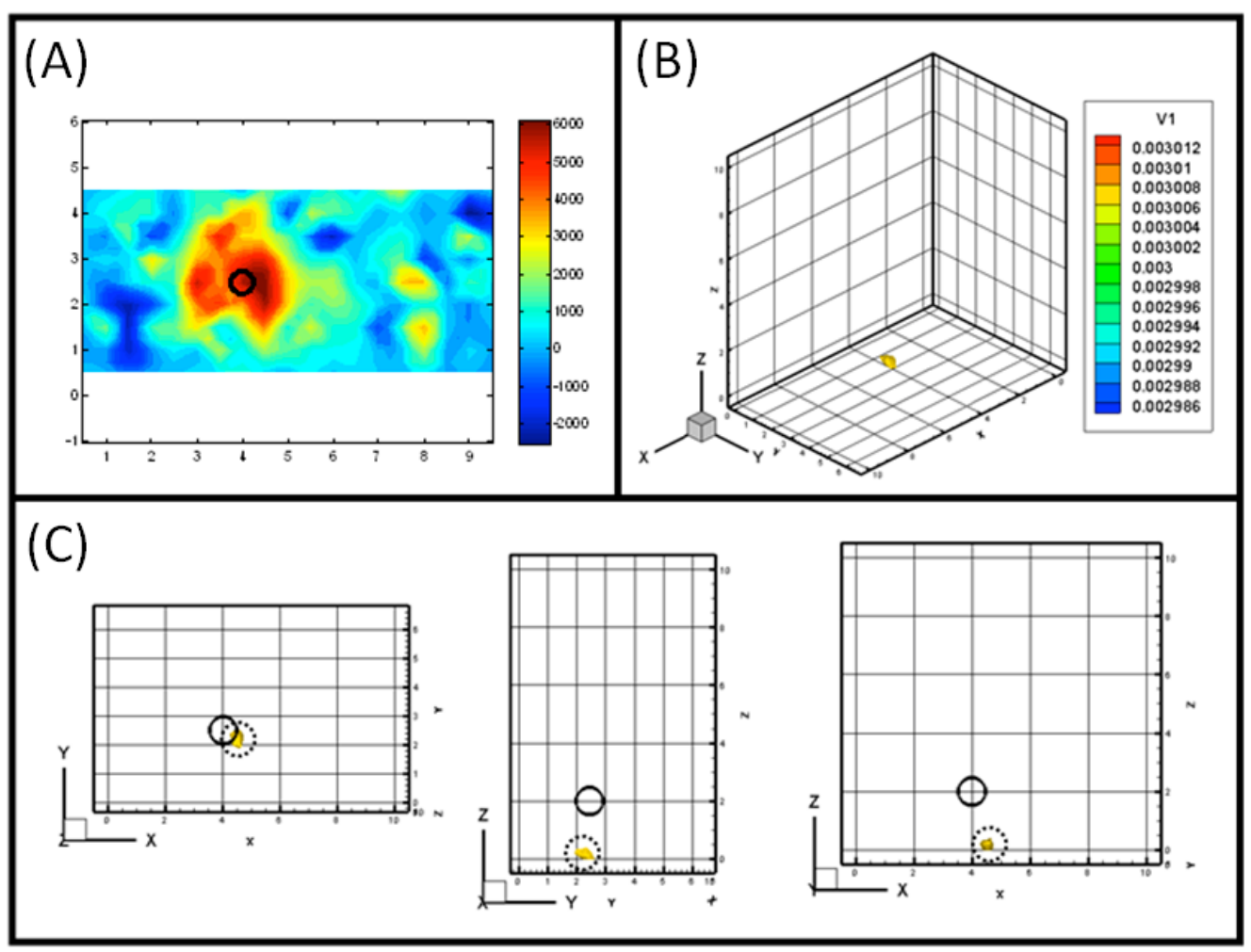

Figure 10.1. Reconstruction of $2.0 \mathrm{~cm}$ deep target within heterogeneous in vitro phantom under perfect uptake case. (A) 2D image collected using hand-held device, (B) 3D view of isosurface plot of reconstructed target, (C) three views of reconstructed target (solid open circle represents true target location; dotted line encircles recovered target). The term V1 in the legend represents the scale for the recovered $\mu_{\text {axf }}$ values in $\mathrm{cm}^{-1}$. 


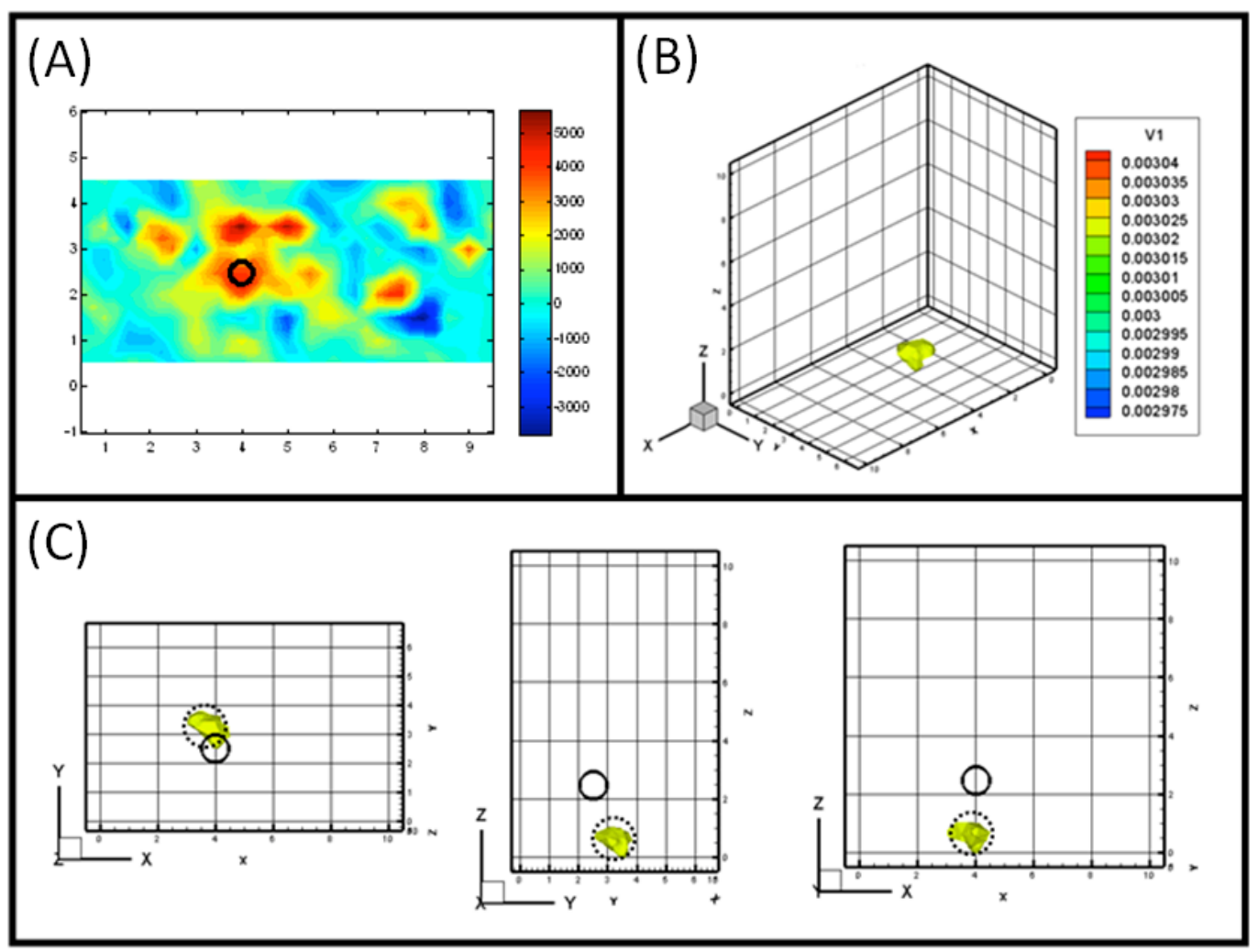

Figure 10.2 Reconstruction of $2.5 \mathrm{~cm}$ deep target within heterogeneous in vitro phantom under perfect uptake case. (A) 2D image collected using hand-held device, (B) 3D view of isosurface plot of reconstructed target, $(\mathrm{C})$ three views of reconstructed target (solid open circle represents true target location; dotted line encircles recovered target). The term V1 in the legend represents the scale for the recovered $\mu_{\text {axf }}$ values in $\mathrm{cm}^{-1}$.

\subsubsection{D CW Based Reconstructions using Summed Multiple-scan Data}

Figure 10.3 shows an initial result from the heterogeneous in vitro phantom with the target located at $2.5 \mathrm{~cm}$ depth from the imaging surface under imperfect (100:1) uptake. 
(A)

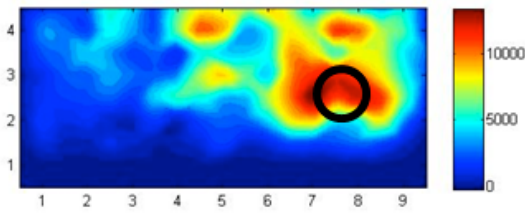

(C)

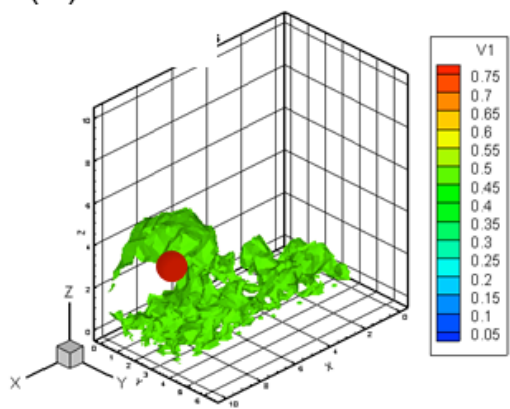

(B)
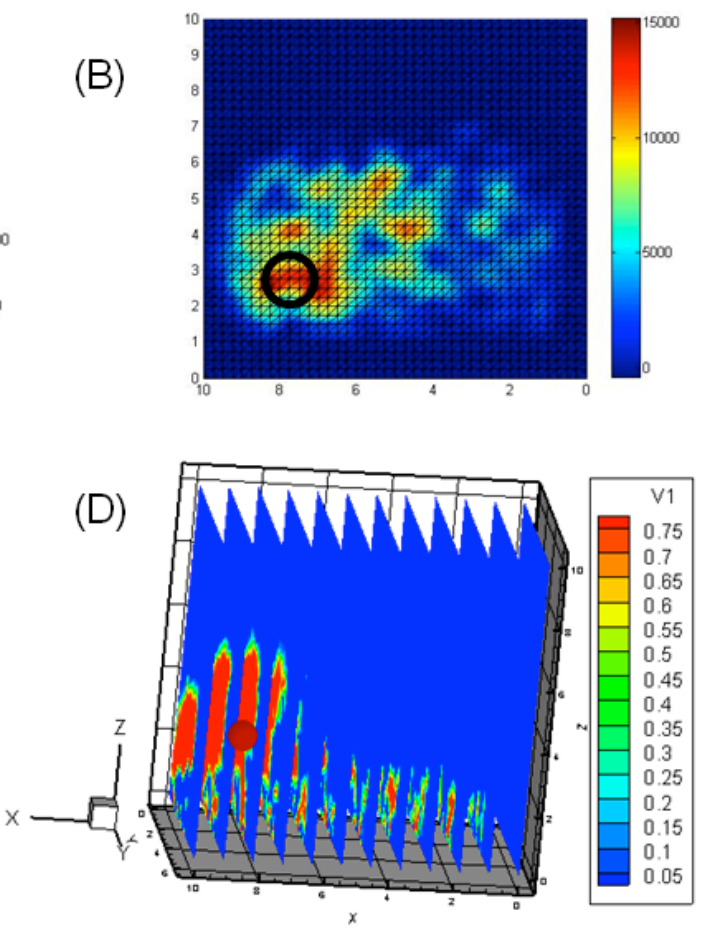

Figure 10.3 3D reconstruction using summed coregistered data. (A) $2 \mathrm{D}$ contour plot of summed images collected using hand-held probe. (B) Coregistered image of summed data with phantom mesh. (C) Isosurface plot of 3D reconstruction. (D) Contour slices showing intensity data of 3D reconstruction. The true target location is indicated by a black circle (A-B) or a dark red sphere (CD).

Figure $10.3 \mathrm{~A}$ shows the $2 \mathrm{D}$ contour plot of fluorescence intensity of the summed image with the true target location indicated by the black open circle. Figure 10.3B shows the coregistered image of the summed data. Figure 10.3C and 10.3D shows the isosurface and contour slices of the $3 \mathrm{D}$ reconstruction respectively. From the contour slices it can be seen that a high signal is reconstructed in the region where the target is located and the reconstructed location is closer to the true depth than previous reconstructions using singlescan FDPM data. There is also noise signal reconstructed close to the imaging surface. This is a result of reflectance-based measurements and in future the first centimeter can be cut off during deep target imaging. 
These preliminary results demonstrated the potential for deeper target recovery upon 3D fluorescence reconstructions using summed multiscan coregistered images. In previous reconstructions using a single image, the target was recovered shallower than the true depth ( $\sim 1 \mathrm{~cm}$ deep). This is due to the use of reflectance based measurements and the same result was found by other researchers as well.[23,105,114] Figure 10.3D shows that the reconstructed signal from the $3.0 \mathrm{~cm}$ deep target is located at a depth greater than $2 \mathrm{~cm}$. In the case of deep target imaging, the first $\mathrm{cm}$ of the image can be cut off to minimize this noise.

Sample results from four of the experimental cases that were reconstructed are presented herein and the details are summarized in Table 10.3. Figure 10.4 shows the reconstruction results (shown as contour slices) from experimental case A where the 8 single scans were reconstructed individually. The true target location is indicated by a grey sphere. It can be seen that the $3.0 \mathrm{~cm}$ deep target was not recovered upon $3 \mathrm{D}$ reconstruction using single scans.

Table 10.3 Summary of selected experimental cases for summated image reconstructions.

\begin{tabular}{cccccc}
\hline Experimental Case & $\begin{array}{c}\text { Target } \\
\text { Volume }\end{array}$ & $\begin{array}{c}\text { Target } \\
\text { Depth }\end{array}$ & T:B & $\begin{array}{c}\text { Target } \\
(\mathbf{x}, \mathbf{y}, \mathbf{z})\end{array}$ \\
\hline $\begin{array}{c}\text { Tissue } \\
\text { Phantom }\end{array}$ & $\mathrm{A}$ & $0.45 \mathrm{~cm}^{3}$ & $3.0 \mathrm{~cm}$ & $100: 1$ & $(4.5,2.5,3.0)$ \\
\cline { 2 - 6 } & $\mathrm{B}$ & $0.23 \mathrm{~cm}^{3}$ & $2.0 \mathrm{~cm}$ & $100: 1$ & $(6.0,2.5,2.0)$ \\
\hline \multirow{2}{*}{ In Vitro } & $\mathrm{C}$ & $0.45 \mathrm{~cm}^{3}$ & $3.0 \mathrm{~cm}$ & $1: 0$ & $(4.5,2.5,3.0)$ \\
\cline { 2 - 6 } & $\mathrm{D}$ & $0.45 \mathrm{~cm}^{3}$ & $3.5 \mathrm{~cm}$ & $1: 0$ & $(4.5,2.5,3.5)$ \\
\hline
\end{tabular}



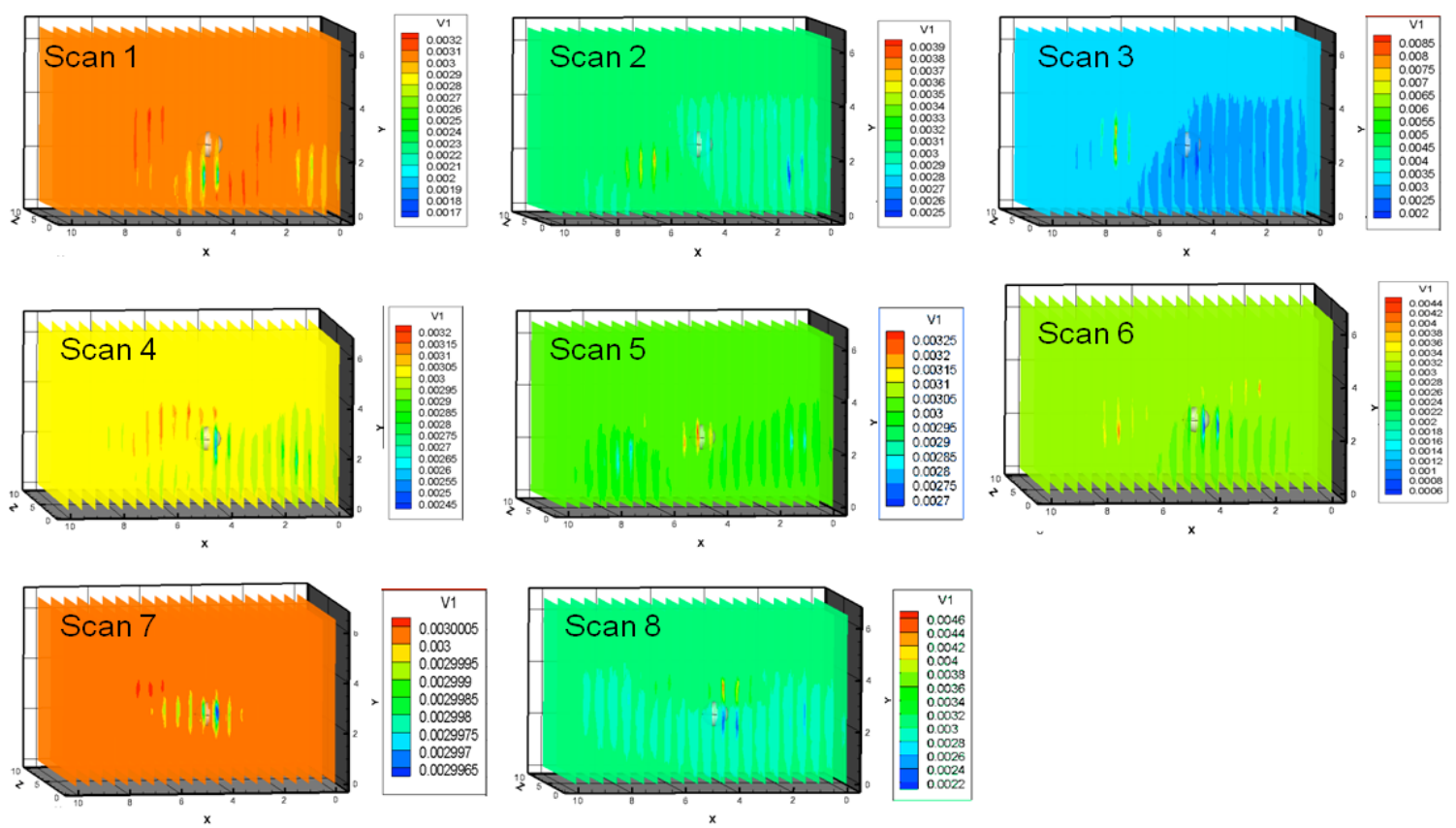

Figure 10.4 Individual 3D reconstructions (shown as contour slices) of the 8 single scans from experimental case A in Table 10.3. The grey sphere represents the true target location.

Figure 10.5 shows results for the first method of CW based summated multiple-scan reconstructions where the recovered $\mu_{\text {axf }}$ values from the individual reconstructed scans were summed together. The results show contour slice plots for the four experimental cases summarized in Table 10.3. In each case, the plots in the left column represent the summation of four scans from four probe locations (i.e. scans $1,3,5,7$ ) and the plots in the right column represent the summation of the repeated set of four scans at the same probe locations (i.e. scans $2,4,6,8)$. 

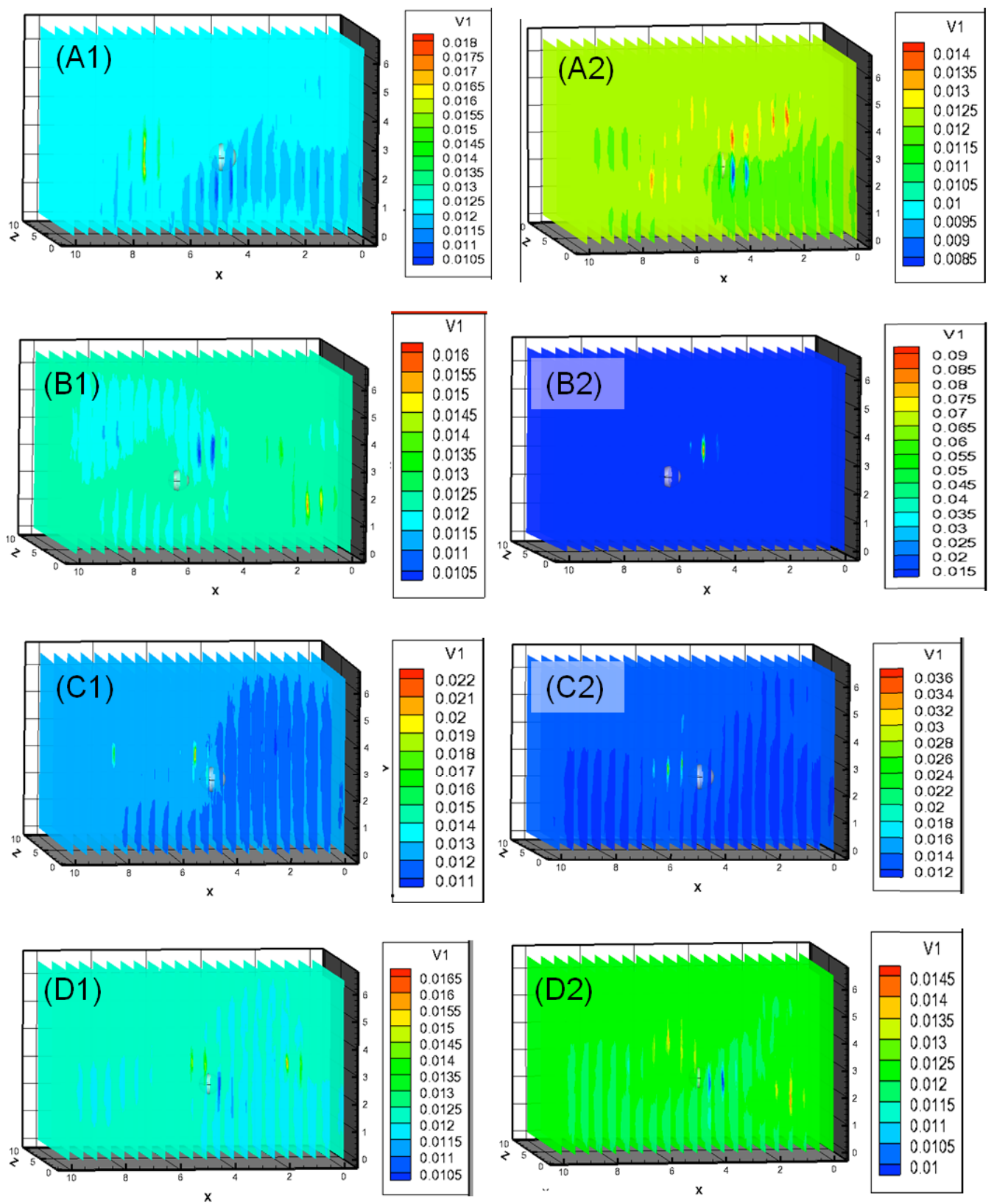

Figure 10.5 Results for the first method of CW based summated multiple-scan reconstruction (shown as contour slices) where the recovered $\mu_{\text {axf }}$ values from the individual reconstructed scans were summed together. The true target location in each plot is indicated by a grey sphere.

Plots B1, C1, and C2 in Figure 10.5 show recovered signal close to the true target location without surrounding artifacts. However, there is no discernible target recovered in the other 
five plots. Hence, it cannot be concluded that a target is recoverable using this method of summation towards 3D tomography.

The results from the second reconstruction method where the $2 \mathrm{D}$ image containing the summed raw data from the entire set of eight scans was used in the $3 \mathrm{D}$ reconstruction are given in Figure 10.6. Figure 10.6A shows the result for a $0.45 \mathrm{~cm}^{3}$ target with $1 \mu \mathrm{M}$ ICG placed $3.0 \mathrm{~cm}$ deep in a tissue phantom under imperfect uptake $(\mathrm{T}: \mathrm{B}=100: 1)$. Figure 10.6B shows the result for a smaller target $\left(0.23 \mathrm{~cm}^{3}\right)$ with $1 \mu \mathrm{M}$ ICG placed $2.0 \mathrm{~cm}$ deep in a tissue phantom under imperfect uptake $(T: B=100: 1)$. Both cases show a region of higher signal close to the true target location (indicated by the yellow dotted circles). In each case, the recovered signal is located vertically higher than the true target location, and there are many surrounding artifacts. Figure $10.6 \mathrm{C}$ shows the result for a $0.45 \mathrm{~cm}^{3}$ target with $1 \mu \mathrm{M}$ ICG placed $3.0 \mathrm{~cm}$ deep in vitro under perfect uptake $(\mathrm{T}: \mathrm{B}=1: 0)$. Figure 10.6D shows the result for a $0.45 \mathrm{~cm}^{3}$ target with $1 \mu \mathrm{M}$ ICG placed $3.5 \mathrm{~cm}$ deep in vitro under perfect uptake $(\mathrm{T}: \mathrm{B}=1: 0)$. In both cases, there is no significant signal recovered close to the true target location. 

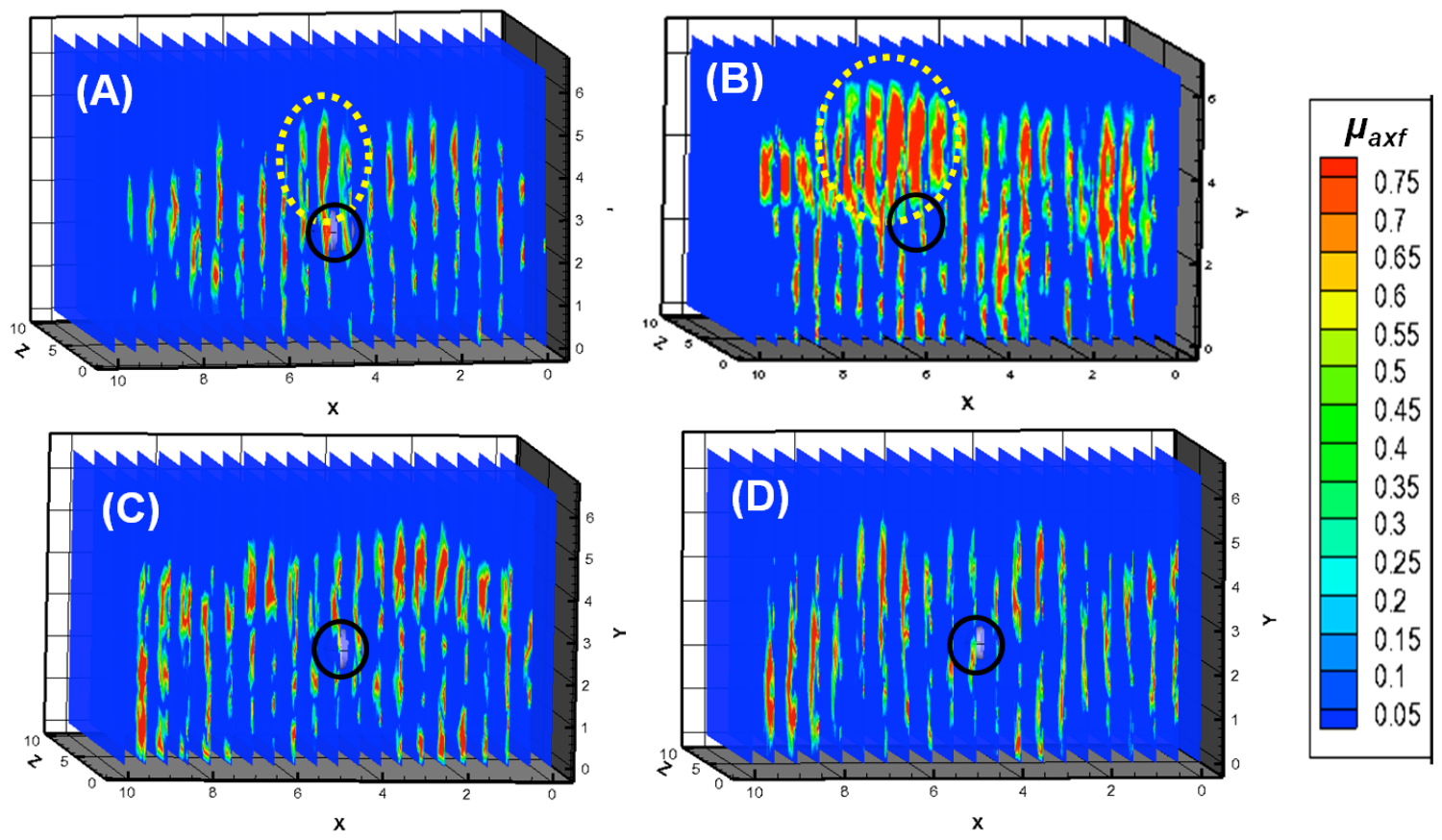

Figure 10.6: Results for 3D reconstructions performed using multiple summated coregistered images. Details of each experimental case are given in Table 10.3. The yellow dotted circles indicate possible recovered signal from the fluorescent targets. The true target locations are indicated by grey spheres circled in black for emphasis.

Overall, the results did not show that reconstruction of summated images enables deeper target recovery. This could be due to several factors. The summed images were treated as a single image at the initial probe location with six source locations and 165 detector locations. An alternate approach would be to incorporate additional source and detector positions at the additional probe locations. However, this would misrepresent the experimental procedure by treating the sequentially collected scans as though they were collected simultaneously with additional sources and detectors. A parallel study was performed where the individual data sets were reconstructed prior to summation and then the recovered $\mu_{\text {axf }}$ values were summed. However, the results did not show reconstruction of the deeper targets upon summation. This was likely due to the high amount of noise and lack of signal at the true target location in each of the individual reconstructed images. In 
the future, some filtration techniques might be applied to reduce the noise in the images possibly enabling reconstruction of the targets using the summated images.

\subsection{SUMMARY AND CONCLUSION}

Studies were performed to demonstrate 3D tomography in tissue phantoms and in vitro to recover a fluorescent target within a heterogeneous scattering background. FDPM based reconstructions were performed in vitro and fluorescent targets were recovered up to a depth of $2.5 \mathrm{~cm}$. CW based multiple-scan reconstructions were performed in tissue phantoms and in vitro toward deeper target recovery using two methods: (i) summation of the recovered $\mu_{\text {axf }}$ values from the individually reconstructed single scans; (ii) performing $3 \mathrm{D}$ reconstruction using the summed raw data from the entire set of scans. The results from the CW based multiple-scan reconstructions did not conclusively show the reconstruction of deeper targets upon using the summated images. Possible filtration techniques can be applied in the future to reduce the noise in the summed images. 


\section{CHAPTER 11}

\section{Study IV : 2D Imaging In Vivo with Healthy Human Subjects in Near- Real Time towards Target Detection}

\subsection{INTRODUCTION}

Thus far, fast 2D imaging, coregistered imaging, and 3D tomography have been demonstrated in tissue phantoms and in vitro. Herein, in vivo studies are performed on healthy human subjects to demonstrate the feasibility of using the hand-held device to image a fluorescent target with a background of real human breast tissue.[156]

\subsection{MATERIALS AND METHODS}

All human subject studies were approved by the Florida International University Institutional Review Board. Healthy female volunteers age 21 and above were recruited for the studies. A spherical fluorescent target was used to simulate a tumor (the same target used in phantom and in vitro studies) and was placed underneath the flap of the breast tissue (i.e. between breast tissue and chest wall, underneath the tissue) as shown in Figure 11.1. Table 11.1 gives a summary of the in vivo experimental cases performed.

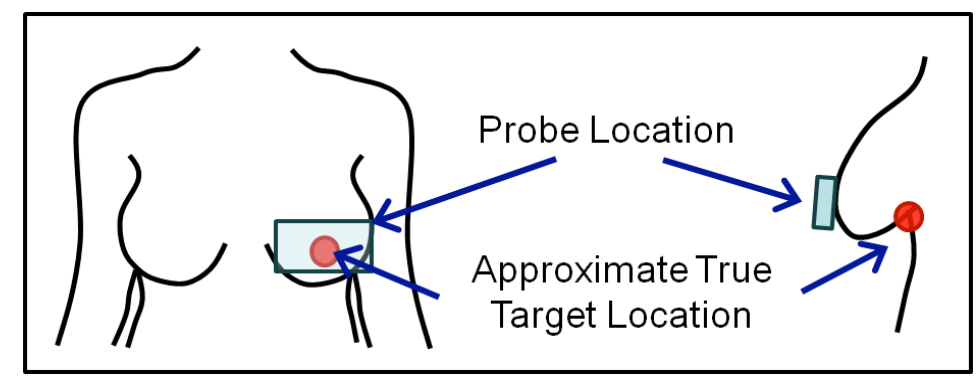

Figure 11.1 Schematic showing the location of an absorbing target placed superficially underneath the breast tissue and the location of the probe during imaging. 
Table 11.1 Summary of experimental cases performed for in vivo fast 2D imaging studies.

\begin{tabular}{|c|c|c|c|c|c|}
\hline \multicolumn{2}{|c|}{ Experimental Case \# } & $\begin{array}{c}\text { Number of } \\
\text { Targets }\end{array}$ & $\begin{array}{l}\text { Target Depth } \\
\text { (cm) }\end{array}$ & $\begin{array}{c}\text { Target } \\
\text { Volume (cc) }\end{array}$ & $\begin{array}{c}\text { T:B Contrast } \\
\text { Ratio }\end{array}$ \\
\hline \multirow{3}{*}{$\begin{array}{l}\text { In vivo with normal } \\
\text { human subject }\end{array}$} & 1 & 1 & 2.5 & 0.23 & $1: 0$ \\
\hline & 2 & 1 & 2.5 & 0.45 & $1: 0$ \\
\hline & 3 & 2 & 2.5 & $0.23 \& 0.45$ & $1: 0$ \\
\hline
\end{tabular}

In the first study, a $0.23 \mathrm{cc}$ sphere with $1 \mu \mathrm{M}$ ICG was placed under the right breast in the 4 o'clock position. The flat probe face was placed against the breast tissue (centered at the 6 o'clock location) with gentle compression to achieve full contact with the tissue and a near-real time $\mathrm{CW}$ fluorescent intensity image was acquired (around the target region). The depth of the target within the tissue was approximately $2.5 \mathrm{~cm}$ as measured with a Vernier caliper. The experiment set-up for in vivo human subject studies is shown in Figure 11.1 (with mannequin for demonstration only).

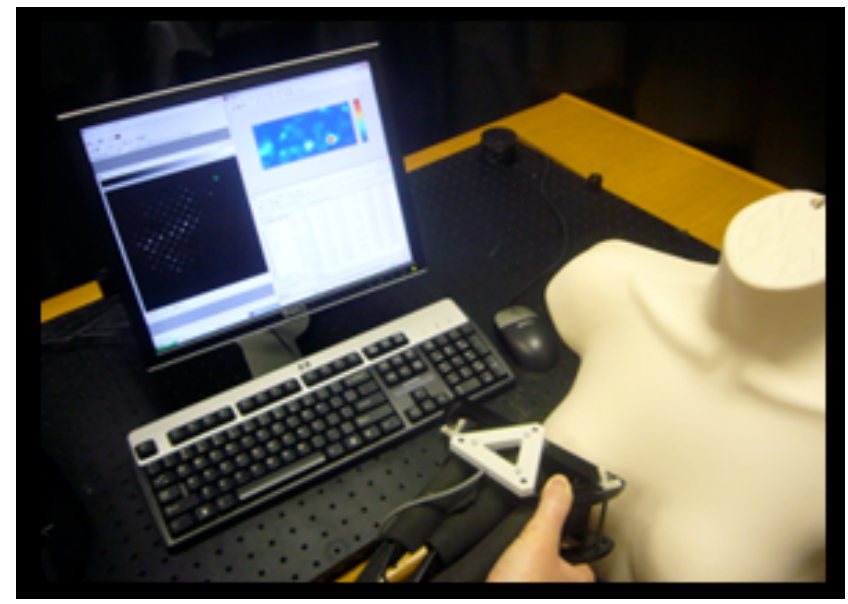

Figure 11.2 Experimental set-up for in vivo studies with human subject (mannequin shown here for demonstration only). The hand-held probe is placed in full contact with the breast tissue. The raw image and the $2 \mathrm{D}$ contour plot are displayed on the computer screen. 
A second study was performed using a single target with the probe in the maximum curved position (i.e. $45^{\circ}$ curvature of the two side plates of the 3 -plate based probe face). The images collected with the probe in the curved position possibly include trans-illuminated measurements in addition to reflectance based measurements. This study was performed to demonstrate the feasibility of using the probe in its curved position, such that it can contour along the tissue and also provide fluorescent images that can aid in target detection. Herein, a $0.45 \mathrm{cc}$ fluorescent target containing $1 \mu \mathrm{M}$ ICG was placed under the right breast in the 8 o'clock position. A real-time as well as fast (subtracted) image of fluorescence intensity was acquired.

A third study was performed to demonstrate the feasibility of imaging multiple targets within real human breast tissue. Two targets were placed under the fold of the left breast tissue, with a $0.23 \mathrm{cc}$ target at the 6 o'clock position, and a $0.45 \mathrm{cc}$ target was placed at the eight o'clock position of the same breast. A real-time as well as fast (subtracted) image of fluorescence intensity was acquired by applying gentle compression on the left breast tissue.

During the studies, the probe was placed visually over the breast tissue and centered with respect to the nipple region. For future in vivo studies with human subjects, a more systematic protocol is currently being developed. The method uses a clear plastic sheet with holes placed on a grid at premeasured locations. The plastic will be placed over the tissue and a pen will be used to mark the probe locations on the tissue. The grid is shown in figure 11.2. 

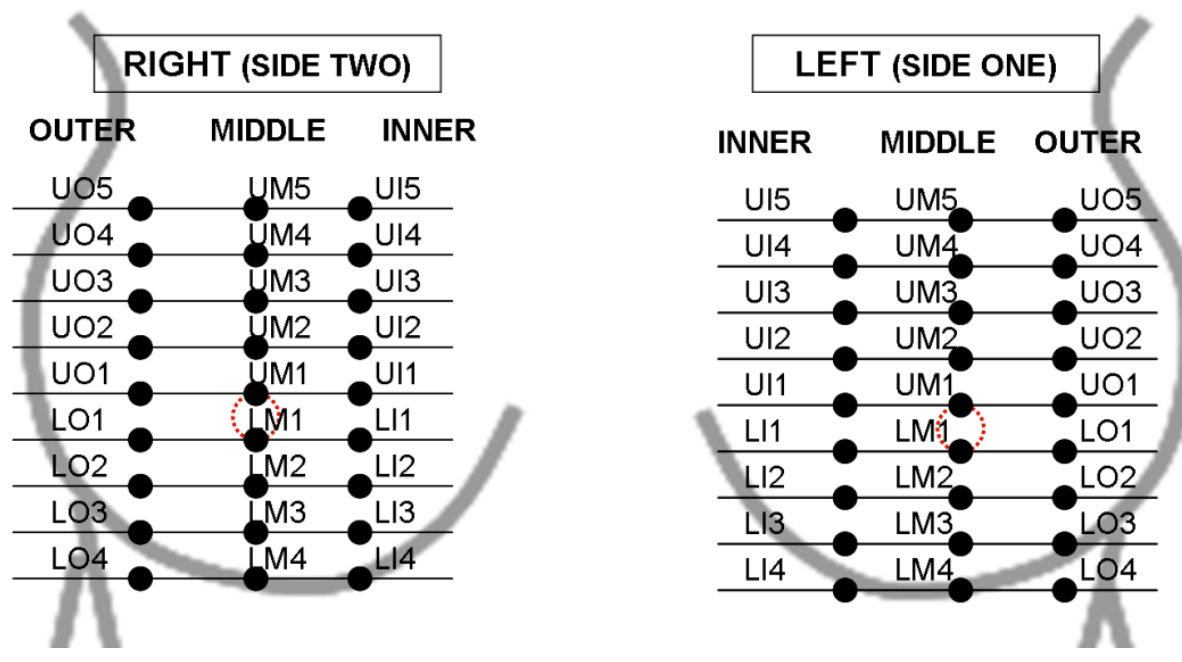

Figure 11.3 Systematic grid developed for future imaging studies. The positions are measured with reference to the center (nipple) region which is indicated by the red dotted circle.

The positions are measured and numbered from the nipple region and the number of locations used will be determined by the volume of tissue to be imaged. For the first set of images, the probe will be centered over the marks in the middle region with the bottom of the probe aligned with each mark. For the second set the probe will be centered over the marks in the outer region, and for the third set the probe will be centered over the marks in the inner region. The positions are labeled with U or L to indicate "upper" and "lower" region, respectively, and $\mathrm{O}, \mathrm{M}$, or I to indicate "outer", "middle", or "inner" region, respectively. The entire set will be repeated on the other breast at the corresponding locations. For the studies described here, the probe location in all cases roughly corresponds to the LM4 location in Figure 11.2. 


\subsection{RESULTS}

Figure 30 shows the fast $2 \mathrm{D}$ subtracted images of fluorescence intensity obtained in vivo (from a normal human subject using a simulated target) with the probe in the flat position (Figure 30A) and in the curved position (Figure 30B). These subtracted image results demonstrate the feasibility of fast $2 \mathrm{D}$ surface imaging and $2 \mathrm{D}$ target localization in a clinical environment. The real-time (non-subtracted) images of fluorescence intensity were unable to differentiate the target from the heterogeneous background, and hence only the fast 2D subtracted images are shown in Figure 11.3.
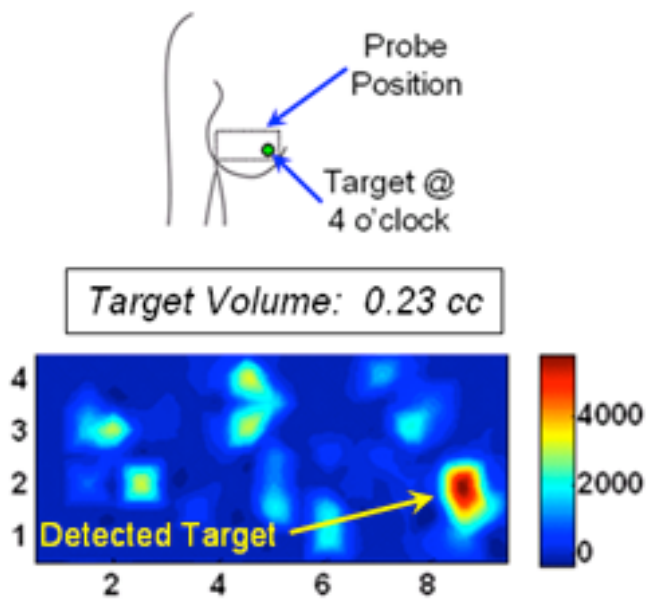

(A)

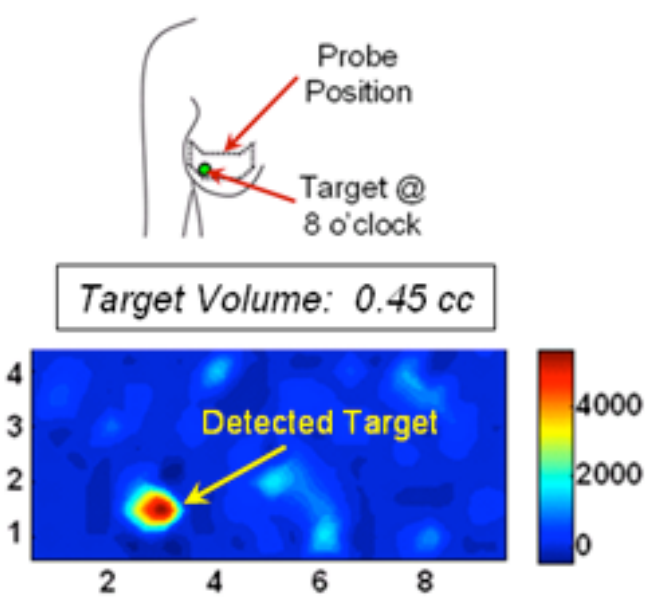

(B)

Figure 11.4 Fast subtracted images of fluorescence intensity obtained as 2D surface contour plots, acquired in vivo from a human subject using a spherical fluorescent target, for two experimental cases: (A) the probe was in the flat position and a $0.23 \mathrm{cc}$ target was placed at the 4 o'clock position, and (B) the probe was in the curved position and a $0.45 \mathrm{cc}$ target was placed at 8 o'clock position. The images acquired using the probe in the curved position are illustrated as projected as a flat $2 \mathrm{D}$ image, in order to be consistent with the images presented in case (A) (i.e. using the probe in flat position). 


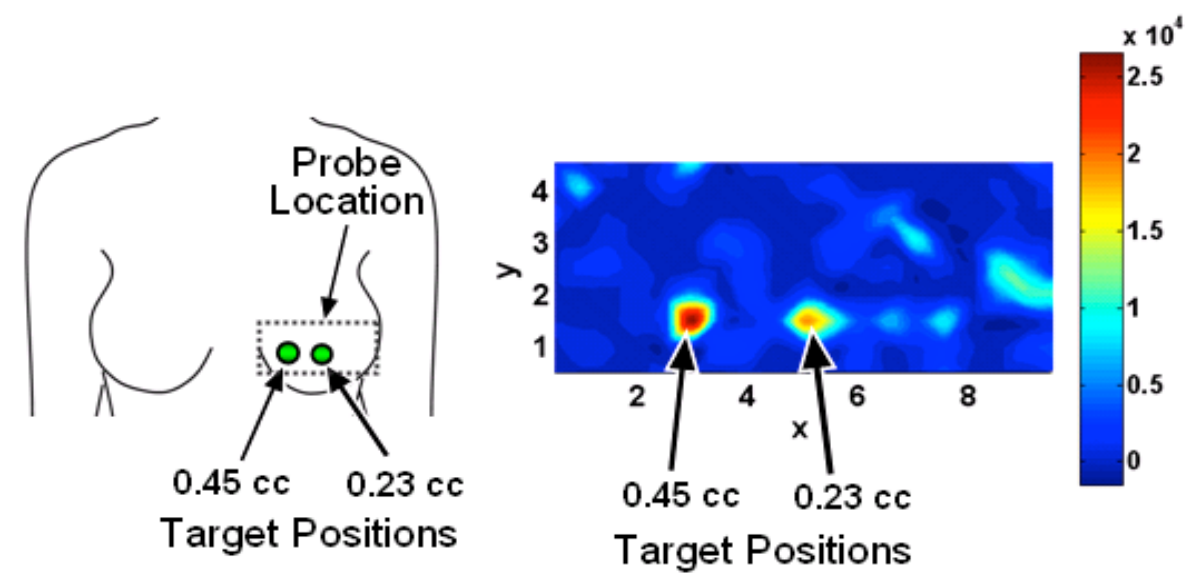

Figure 11.5 Fast subtracted image of fluorescence intensity obtained as $2 \mathrm{D}$ surface contour plot, acquired in vivo from a human subject using two spherical fluorescent targets $(0.23$ and $0.45 \mathrm{cc})$.

The 2D subtracted images of fluorescent intensity from multiple simulated targets in a human subject are shown in Figure 11.4. The $0.23 \mathrm{cc}$ target is detected in the center of the image and the $0.45 \mathrm{cc}$ target is detected towards the left side in the image, which are very close to the true locations of these targets. This study demonstrates the potential to image and localize multiple fluorescent targets (of different sizes) within human breast tissue.

\subsection{DISCUSSION AND CONCLUSION}

The fluorescence imaging studies described here demonstrate for the first time the acquisition of fast $2 \mathrm{D}$ surface images (in $<5 \mathrm{sec}$ ) of a fluorescent target in vivo using a handheld based optical imaging device. The subtracted images have a potential to clearly differentiate target(s) from the background of human breast tissue at a depth of $\sim 2.5 \mathrm{~cm}$ either with the probe in the flat position using gentle compression or with the probe in the curved position to contour around the tissue curvature. The results demonstrate the potential to translate the technology towards on-site breast imaging in a clinical environment. 


\section{CHAPTER 12}

\section{Study V: Coregistered Imaging In Vivo with Healthy Subjects}

\subsection{INTRODUCTION}

In order to perform 3D tomography in complex geometries such as human breast tissues, it is necessary to accurately coregister the image to the tissue geometry. In the phantom studies described above, the simple cubical geometries were generated manually using Gambit software. In the case of human subjects, the geometry shape and volume varies for each subject and thus must be acquired individually. This chapter describes the process of acquiring the breast tissue geometry for each human subject, generating the surface mesh of the tissue geometry, and demonstrating coregistered imaging on normal human subjects.

\subsection{MATERIALS AND METHODS}

\subsubsection{Initial coregistered imaging approach (motorized 3D scanner \& sitting position)}

A commercially available hand-held 3D scanner (Polhemus, Inc.) was employed to acquire the breast tissue geometry. Use of the scanner on human subjects was approved by the FIU Institutional Review Board. A motorized system was implemented with scanner to automatically acquire the scan without requiring an operator to hold the scanner. This method was designed to enable the scanning procedure to be independent of the operator as well as to allow more privacy for the subject since the scanner would be operated by computer from outside a curtained room. The automated scanning system set-up is shown in Figure 12.1A where the subject was seated in an upright position during the scan (mannequin is shown for demonstration only). Each scan was composed of a series of four 
sweeps of the scanner which would cover both sides of the breast tissue as well as part of the chest wall and ribcage area. The acquired geometry was displayed by the
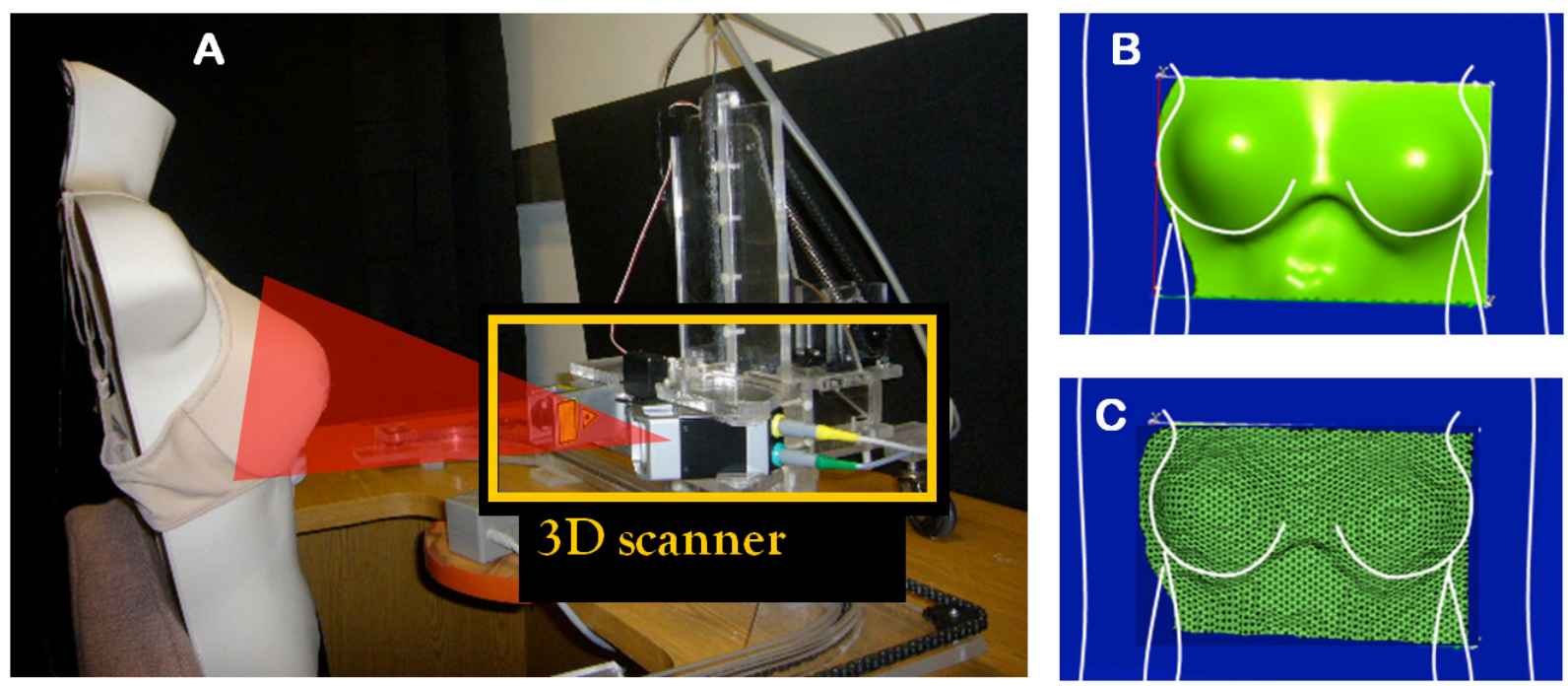

Figure 12.1 Initial set-up for acquiring 3D breast tissue geometry for coregistered imaging.

Polhemus software (Figure 12.1B) and then exported as a .MAT file and discretized using MATLAB software developed in house to generate the surface mesh geometry to be loaded into the coregistration software (Figure12.1C). The automated coregistered imaging process using MATLAB/LabVIEW software developed in house was detailed in Chapter 6.

\subsubsection{Validation of coregistered imaging in human subjects}

Automated coregistered imaging has been demonstrated in phantoms which have rigid structure and simple geometry. Translation of this procedure to human tissue is a much more complex case since human tissue is not rigid and has irregular shapes. Prior to in vivo studies on human subjects, it is necessary to validate the automated coregistered imaging process on actual human tissue. 
Coregistered imaging in human breast tissue is validated by the following procedure. In step one the breast tissue geometry is acquired by using a 3D scanner to scan the breast tissue (Figure 12.2A). In step two, the 3D geometry is transformed into a discretized surface mesh (Figure 12.2B). In step three, an initial reference point is automatically inserted into the mesh at the center region of the tissue which is marked correspondingly at the same location on the subject (Figure 12.2C). Four additional reference points are generated automatically and the $x-y-z$ positions are given quantitatively relative to the initial reference point (Figure 12.2D).

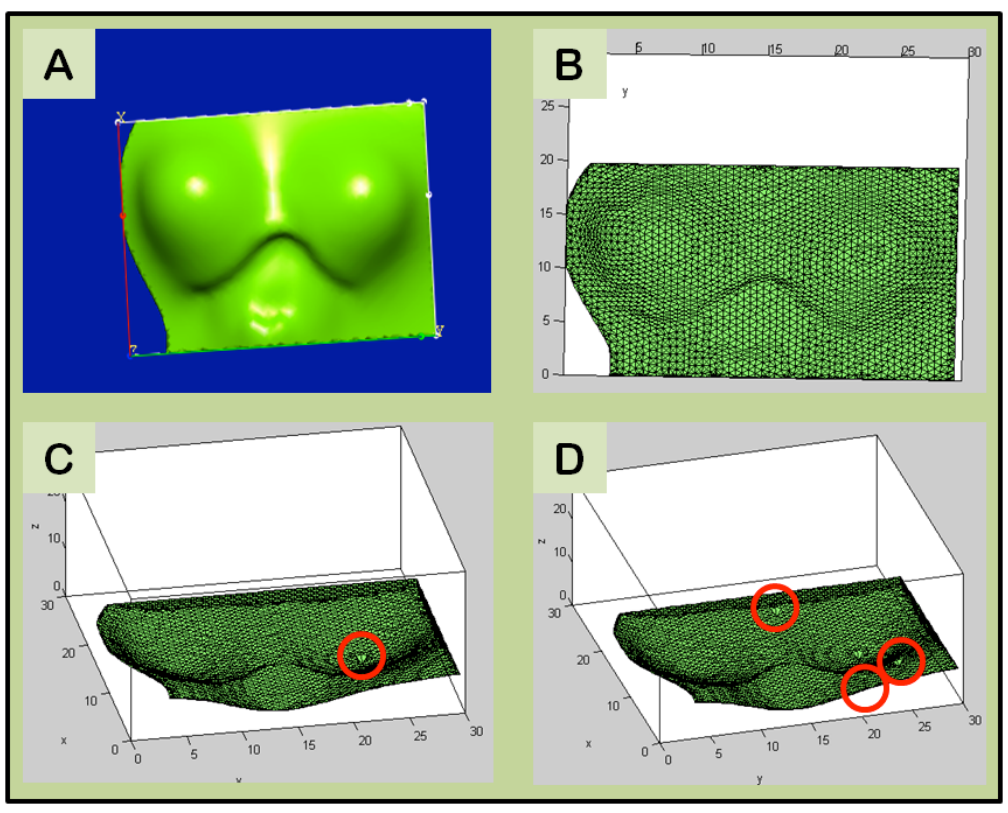

Figure 12.2 Automated input of reference points within any 3D scanned geometry.

In step four, the locations of the additional reference points are measured on the subject and marked with surgical tape (Figure 12.3A). In step five the probe is positioned such that the origin is located at the initial reference point on the subject (Figure 12.3B-C) and the 
coregistration software is initialized (Figure 12.3D). In step six, the probe is moved to other positions marked by the additional reference points and the coordinates of the probe location given in the tracking software are compared to the known coordinates of the reference points marked on the subject to validate that the image is coregistered onto the true location of the tissue.

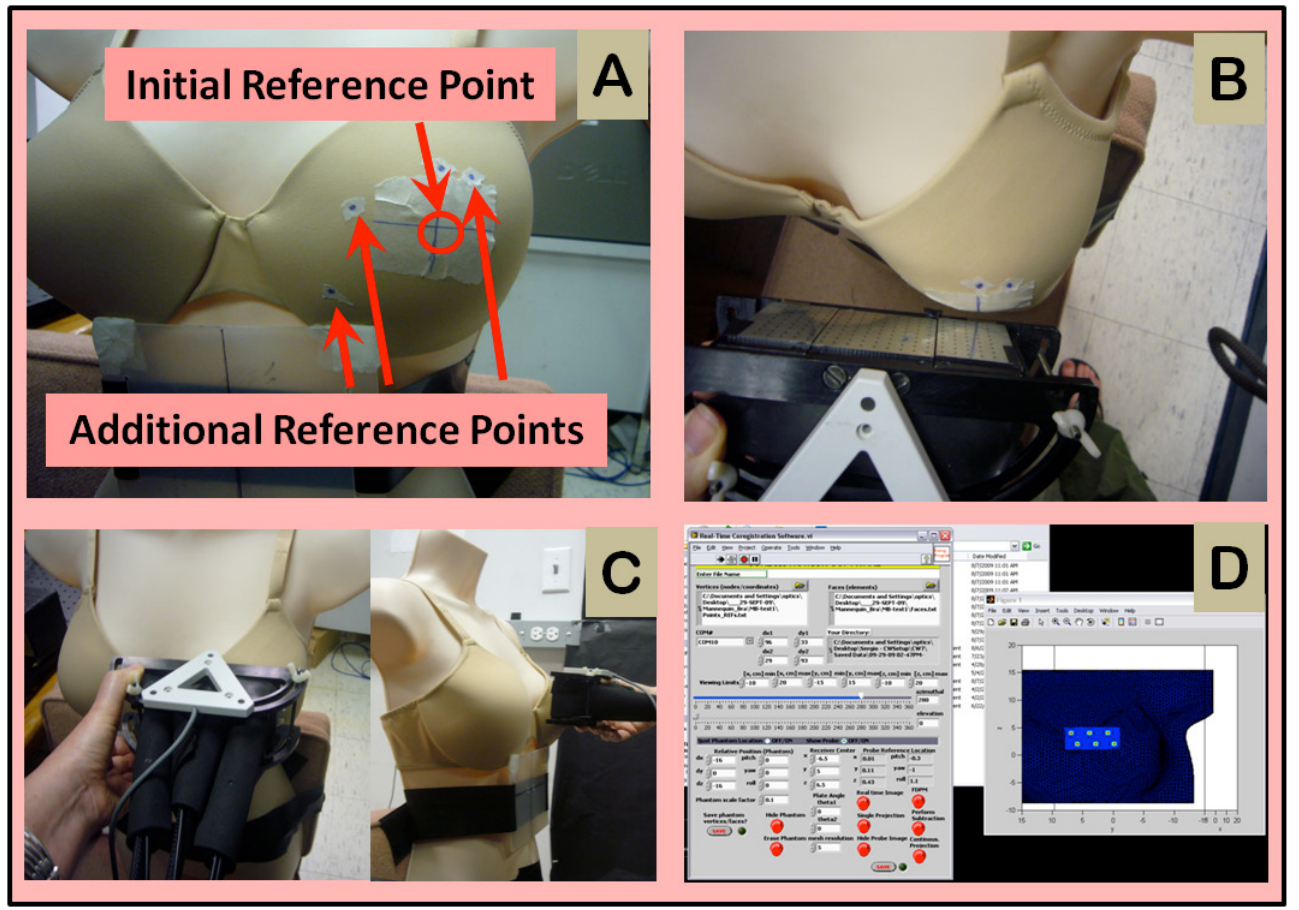

Figure 12.3 Placement of reference points on the subject and initialization of coregistration software.

In order to quantitatively determine the accuracy of the tracked position and coregistered image compared to the true location on the tissue, the probe was placed at the four different reference points of known coordinates and ten measured values were recorded from the tracking software at each position. The average, standard deviation, and 
discrepancy of these measured values for each position are given in Table 12.1. The average measured value and standard deviation represent the precision of the tracking software.

Table 12.1 Average and standard deviation of $x, y, z$ coordinates as displayed in tracking software and total distance off between true and measured values. Values are in units of centimeters and each measured value represents an average of 10 measurements while probe was at each of the 4 positions.

\begin{tabular}{ccccc}
\hline & $\begin{array}{c}\text { True Value } \\
(x, y, z)\end{array}$ & $\begin{array}{c}\text { Average Measured } \\
\text { Value } \\
(x, y, z)\end{array}$ & $\begin{array}{c}\text { Standard Dev. of } \\
\text { Measured Value } \\
(x, y, z)\end{array}$ & $\begin{array}{c}\text { Total Distance Off } \\
(\mathrm{cm})\end{array}$ \\
\hline \hline Initial Position & $(0,0,0)$ & $(-0.18,-0.38,0.31)$ & $(0.09,0.08,0.10)$ & 0.53 \\
\hline Position \#2 & $(0,-10,0.5)$ & $(-0.87,-9.19,1.12)$ & $(0.18,0.33,0.27)$ & 1.34 \\
\hline Position \#3 & $(0,-20,0)$ & $(-0.44,-19.58,-0.15)$ & $(0.20,0.31,0.41)$ & 0.63 \\
\hline Position \#4 & $(1,-9,-8)$ & $(0.29,-9.95,-8.87)$ & $(0.06,0.15,0.07)$ & 1.47 \\
\hline
\end{tabular}

It can be seen from the standard deviation of the measured values that the tracked probe position fluctuates between $0.1-0.4 \mathrm{~cm}$. The average total distance off represents the accuracy of the tracked position compared to the true location. The average total distance off is $0.99 \mathrm{~cm}$. The deviation and discrepancy of the measured values can be attributed to several factors: (i) The inherent fluctuation within the tracking instrumentation; (ii) Slight movements of the operator while holding the hand-held probe in position; (iii) Small errors in the measuring and placement of the physical reference points marked on the human subject. In future, efforts will be made to overcome these limitations and improve the accuracy of the tracking coordinates.

A study was performed with a normal human subject to demonstrate selfcoregistered imaging on actual human tissue. Figure 12.4 shows the front panel for the coregistration software and the image of the meshed tissue geometry with the reference 


\section{xyz locations of reference points}

points (probe position is not shown here). Once the reference points are added to the mesh, Distance in $\mathrm{cm}$ of each

a text filefogetnereed and salvedn wich contains the $\mathrm{x}, \mathrm{y}, \mathrm{z}$ coordinates of the reference points

initial reference point

and the relative distance of each reference point from the initial reference point which is

used to place the markers at the corresponding location on the subject.

geometry with

reference points

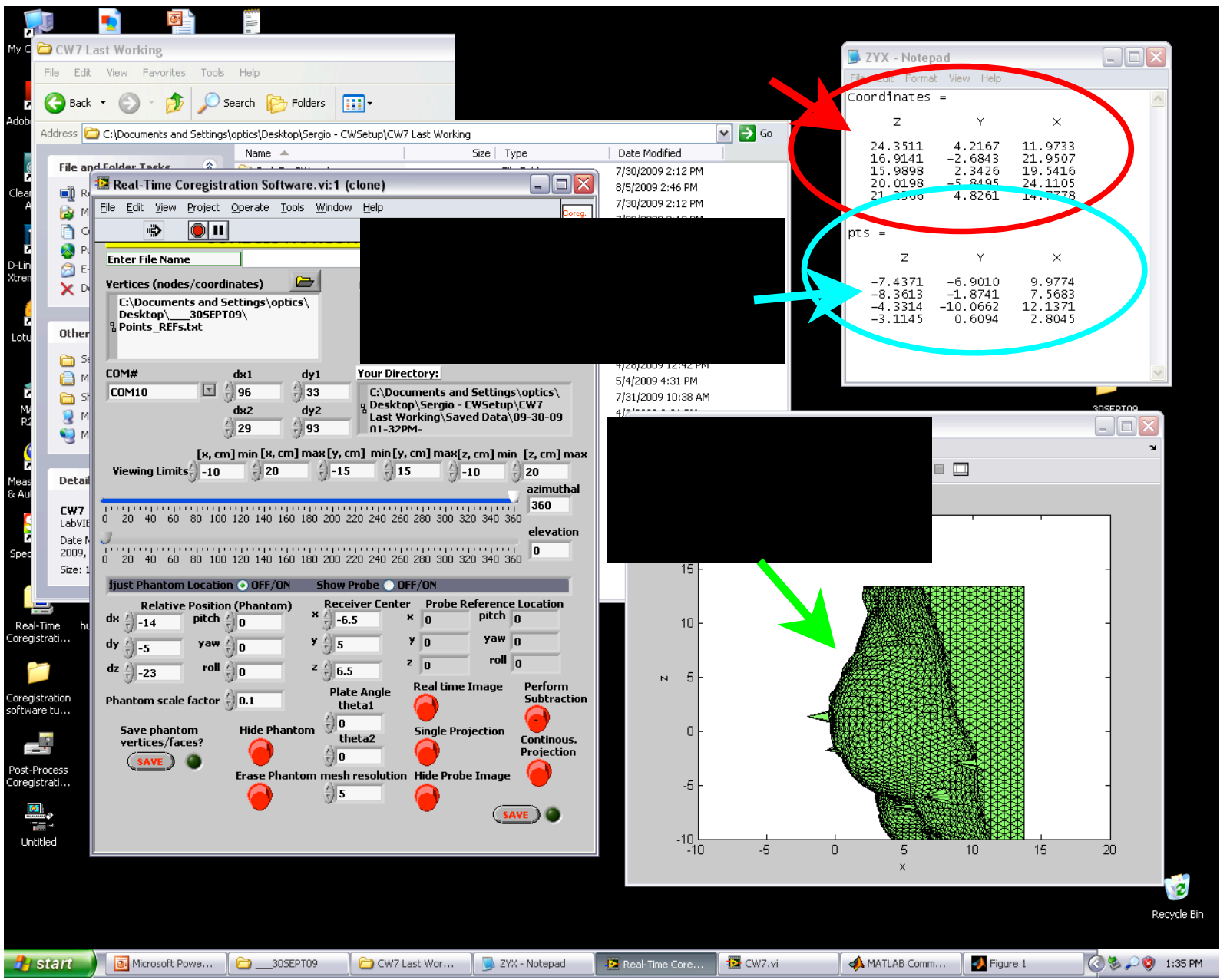

Figure 12.4 Screen capture of coregistration software showing meshed geometry of breast tissue with reference points (probe location inactive in this view).

The probe was placed at the initial reference point location and the coregistration software was initialized. Figure 12.5 shows the tracked probe position at the initial point (the origin) and at two additional reference points as it was moved to the corresponding 
positions marked on the subject. Images are shown in profile view from the subject's left side.

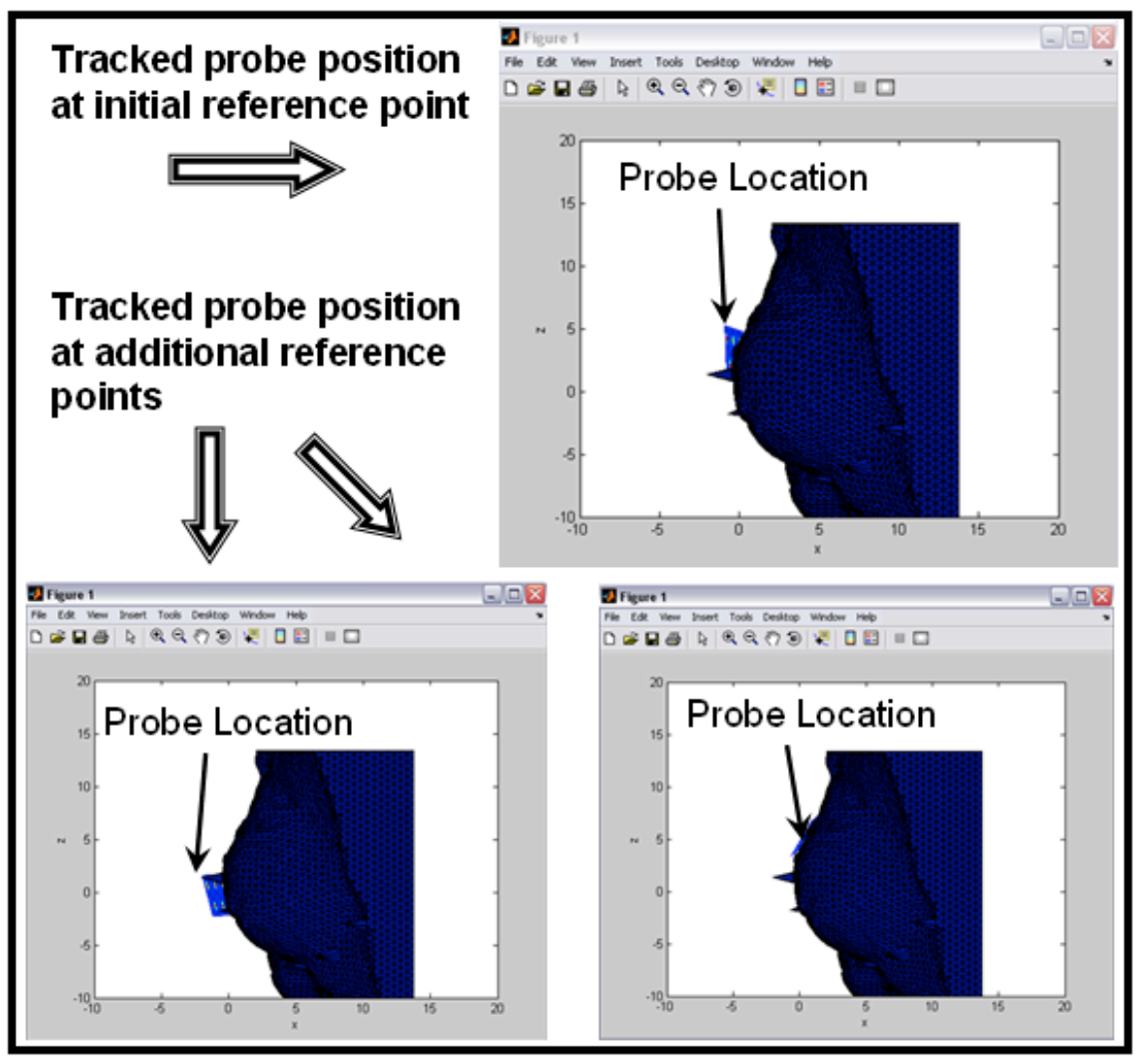

Figure 12.5 Images from coregistration showing probe position tracked in real-time at different reference locations.

In order to perform coregistered imaging, a $0.45 \mathrm{cc}$ spherical target filled with $2 \mu \mathrm{M}$ ICG was placed underneath the flap of the breast tissue (as described in section 11.2) in the center. The probe was placed in full contact with the tissue without applying pressure and an image was collected. The target was then removed while keeping the probe in the same position and a background image was collected. The background image was subtracted from the target image and the subtracted image was coregistered to the meshed geometry of the breast tissue at the probe location. The resulting coregistered image is shown in Figure 12.6. 


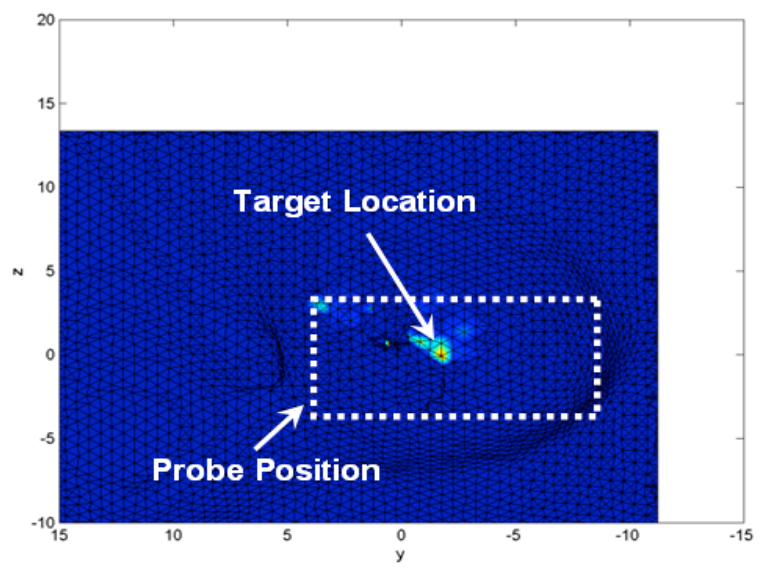

Figure 12.6 Coregistered image of simulated target behind human breast tissue. White dotted line represents the probe position.

This initial study demonstrates the ability to track and coregister an image to the location within the tissue geometry. Some limitations include slight movements of the subject which are not measured or accounted for in the tracking software and could affect the accuracy of the coregistered position, and slight conformation of the tissue upon contact by the hand-held probe face. These limitations were addressed by modifying the imaging set-up as described in the next section.

\subsubsection{Modification of coregistered imaging in vivo to improve accuracy (hand-held 3D scanning and imaging in supine position)}

The initial set-up for coregistered imaging in vivo with human subjects had several limitations which resulted in inaccuracy of tracking and coregistration at the true probe location. The following limitations and proposed solutions were presented:

(1) Limitation \#1: Movement of subject during imaging process which is not accounted for in coregistration software. 
Proposed Solution: A chair will be acquired for the human studies, which has the ability to recline to a 45-90 degree angle to prevent subject from leaning. Once positioned in the chair in a relaxed posture, the subject will be scanned with the $3 \mathrm{D}$ scanner and then they will remain in same position to be imaged with the hand-held probe.

(2) Limitation \#2: Deformation of the breast tissue upon contact with the hand-held probe causing the geometry of the tissue to be changed from the 3D scanned geometry.

Proposed Solution: When the subject is lying at a 45-90 degree reclined positioned, the breast tissue will naturally flatten against the chest wall. Additionally, surgical tape will be used to tape the breast tissue in a flattened conformation similar to the procedure used during ultrasound-guided biopsy. This will enable the probe to be in contact with the tissue with minimal deformation since the tissue will remain generally flat.

(3) Limitation \#3: Operator error in measurement and placement of markers on the subject at the reference points.

Proposed Solution: An optically clear plastic covering can be placed over the breast tissue. The coronal-view coordinates of the reference points can be more accurately marked on the plastic before it is placed over the tissue surface at the appropriate position.

The following steps were implemented in the modified experimental study set-up in order to address the limitations listed above: 
Solution for Limitation \#1: A massage chair capable of reclining $45-90^{\circ}$ with arm rests was acquired for the imaging studies (Figure 12.7). The subjects rested in supine position on the chair with the back reclined at $45^{\circ}$ and arms resting at the sides or on the armrests. This position was chosen such that it would be reclined enough to minimize movement of the subject (as opposed to the upright position with no armrests in the initial set-up which allowed movement of the subject) as well as maintain line-of-sight between the probe and the tracker which would be inhibited if the chair was reclined to greater angles. In order to acquire the 3D geometry with the subject in the reclined position, the Fastscan was taken back as a hand-held scanner and used to scan the tissue by hand (the motorized system will be used in future applications described in Chapter 16).

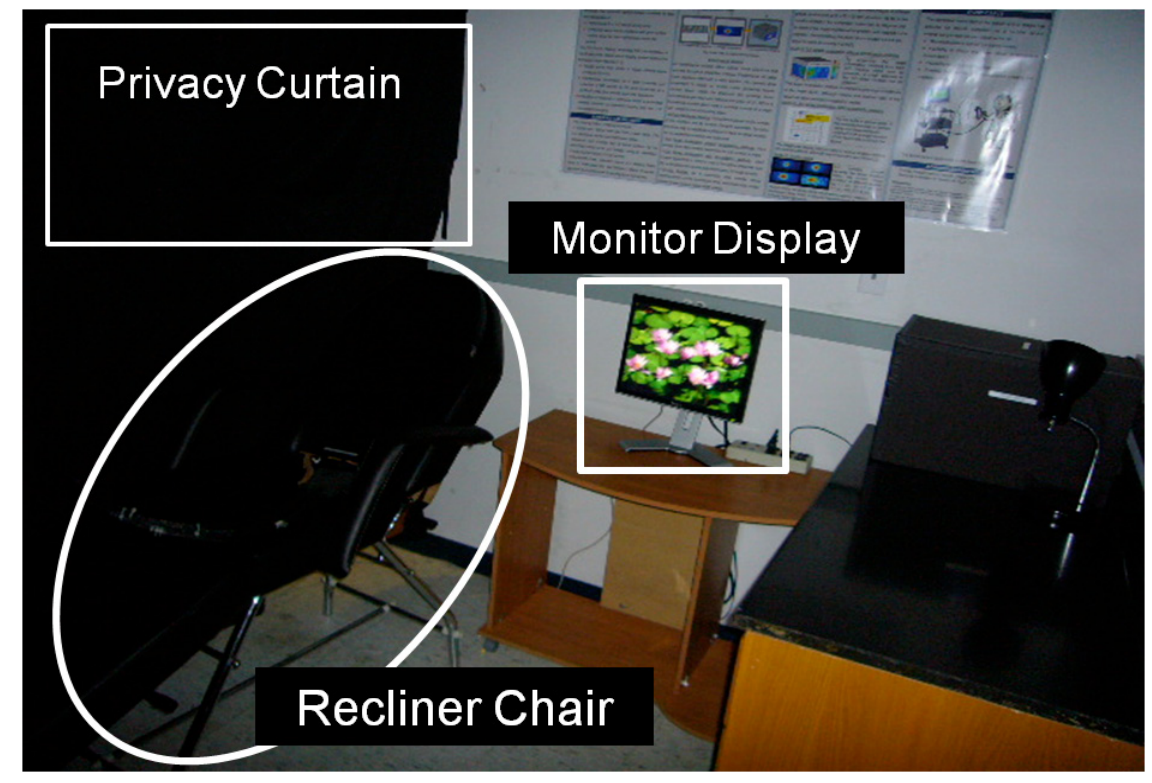

Figure 12.7 Modified human subject imaging set-up. Subject lies supine in recliner chair $\left(45^{\circ}\right)$ during acquisition of tissue geometry with 3D scanner and imaging using handheld imager.

Solution for Limitation \#2: The reclined position of the subject also helped to overcome limitation \#2 since the position caused the breast tissue to flatten against the chest wall 
resulting in minimal conformation of the tissue by the probe during imaging. However, the amount of tissue curvature and deformation depended on the volume and density of the tissue, thus the amount of deformation varied among subjects. (This issue will be addressed in future by use of a custom-built garment described in Chapter 16).

Solution for Limitation \#3: For the initial studies with normal human subjects, the subjects were recruited by advertisement and hence the size and shape of the tissues imaged varied greatly. Hence, it was not feasible at that stage to use a prepared plastic with the reference points marked. Instead, a more accurate approach of choosing reference points was implemented in which the center (nipple) was used as the initial reference point and other reference points were measured and marked at specific distances from the center using the 'clock' notation. This method treats the breast as the face of a clock and locations are determined by their respective location on the clock (Figure 12.8 left) and the distance from the nipple is measured in centimeters (Figure 12.8 right). This method is typically used in the clinical setting during breast ultrasound imaging.

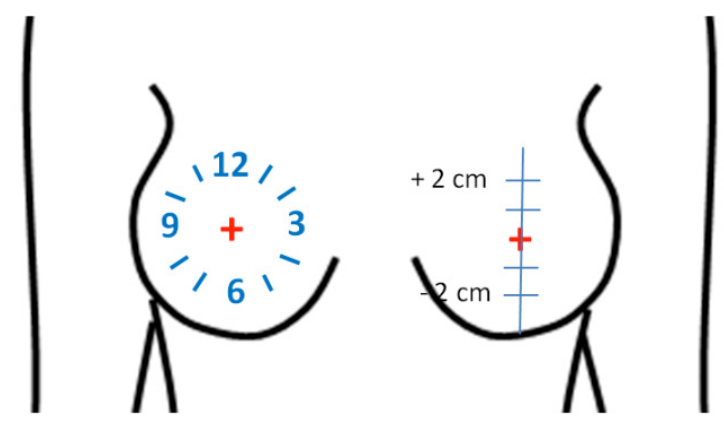

Figure 12.8 'Clock' method for referencing locations on the breast tissue. The relative section of the tissue is determined by the position on the 'clock' (shown on left side) and the distance from the nipple is measured in centimeters (shown on right side). 
The imaging process using the modified set-up is shown in Figure 12.9, where the imaging room is closed off from the instrumentation room by a curtain and only the probe is visible in the room. The probe was placed against the breast tissue while the location was recorded by the acoustic tracker and the images were automatically coregistered at the probe location relative to the tissue geometry. The resulting images were immediately displayed on a monitor in the imaging room that was linked to the computer operated from the instrumentation room.

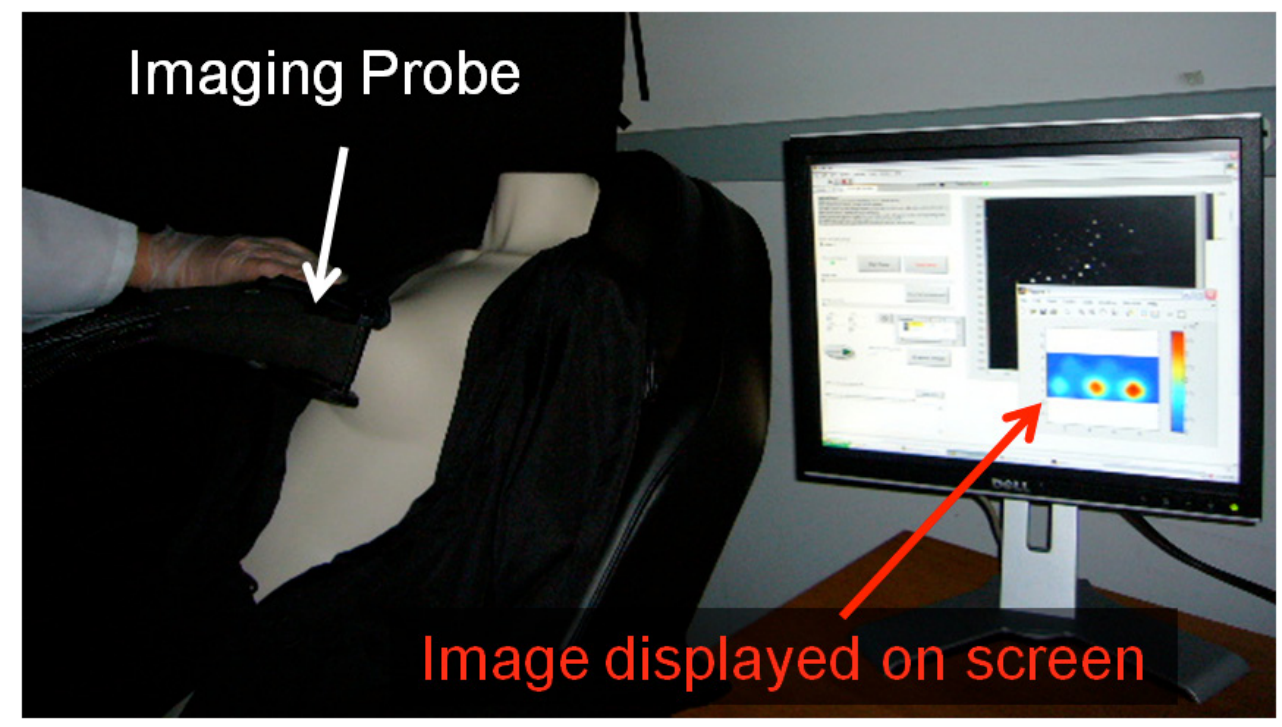

Figure 12.9 Modified imaging set-up. The subject lies in supine position in recliner chair while breast tissue is imaged using hand-held optical imager. Mannequin shown for demonstration only.

\subsubsection{Experimental Study I: Automated Coregistered Imaging using Initial Imaging Set-up}

Experiments were performed using the initial coregistered imaging approach where the subject was seated upright at a $90^{\circ}$ angle. A $0.45 \mathrm{~cm}^{3}$ spherical target filled with 1 $\mu \mathrm{M}$ ICG was placed underneath the flap of the breast tissue as illustrated in Figure 
11.1. Images were collected with the target at 3 different locations corresponding to the 4 o'clock, 6 o'clock, and 8 o'clock positions. In each case, the probe was centered over the target location and placed in full contact with the breast tissue. CW images were collected with the target in place and background images were collected (with the target removed) for subtraction based post-processing. The post-processed images were automatically coregistered during live imaging at the probe location with respect to the tissue.

\subsubsection{Experimental Study II Automated Coregistered Imaging using the Modified Imaging Set-up}

Experimental studies were performed with healthy human subjects using the modified coregistered imaging set-up. The subject lay in the supine position in the recliner chair at a $45^{\circ}$ angle. The chest region including both breast tissues and the ribcage was scanned by hand using the 3D scanner. Figure 12.10 shows the $3 \mathrm{D}$ scanned geometry of a normal human subject using the modified set-up where the subject was lying in supine position in a recliner chair at a $45^{\circ}$ angle. The reclined position caused the tissue to flatten against the ribcage in order to minimize deformation of the tissue during imaging. A default image was used in this study since the purpose of the study was to demonstrate coregistered imaging using the modified approach and not to detect a target. The probe was placed in contact with the breast tissue and the default image was coregistered to the tissue geometry at the probe location. 


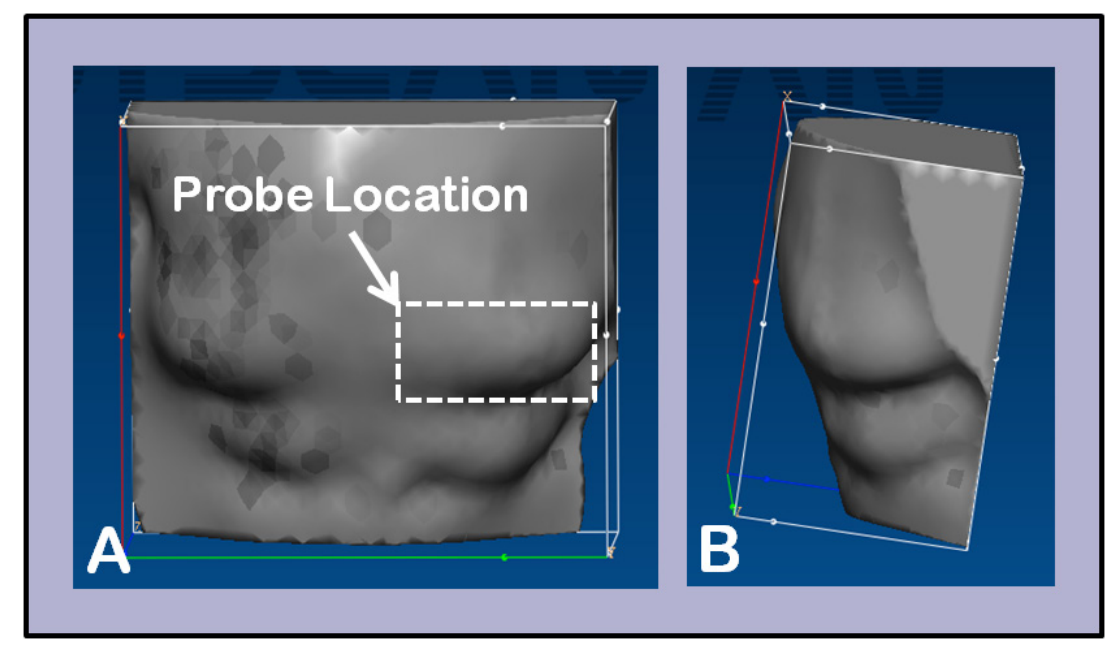

Figure 12.10 Geometry acquired using the modified imaging set-up with a healthy subject lying supine in recliner chair with $45^{\circ}$ angle. The white dotted line represents the probe location during imaging. (A) Frontal view. (B) Side view.

\subsubsection{Experimental Study III Manual Coregistered Imaging using the Modified Imaging Set-up}

Absorption based coregistered imaging studies were performed using a target containing India ink as an absorbing contrast agent. Manual coregistered imaging was performed since the instability of the acoustic based tracker had increased in time to the point where it was no longer usable for coregistration purposes. For these studies, the probe position was measured on the breast tissue and marked using surgical tape. The image was then manually coregistered at the measured position using post-processing coregistration software. Two experiments were performed using $0.45 \mathrm{~cm}^{3}$ spherical targets filled with different concentrations of India ink $(0.08 \%$ and $0.02 \%)$ placed underneath the flap of the breast tissue at the 6 o'clock location. 


\subsection{RESULTS}

\subsubsection{Experimental Study I: Automated Coregistered Imaging using Initial Imaging Set-up}

Figure 12.11 shows results for coregistered imaging studies with healthy subjects using the initial set-up. Figure 12.11A shows the position of the probe and the discretized geometry relative to the subject. Figure $12.11 \mathrm{~B}$ shows the resulting coregistered image for case \#1 where a $0.45 \mathrm{~cm}^{3}$ target was placed at the 6 o'clock position. A zoomed image of the same result is shown in Figure 12.11C. Figures $12.11 \mathrm{D}$ and 12.11E show zoomed images of the results for cases \#2 (0.45 $\mathrm{cm}^{3}$ target at the 8 o'clock position) and \#3 (0.23 $\mathrm{cm}^{3}$ target at the 4 o'clock position) respectively. In each case, the probe was placed such that the strongest source was close to the target location and subtraction based postprocessing was applied to eliminate excitation leakage (as described in Section 6.4 above). The results show that a $0.23-0.45 \mathrm{~cm}^{3}$ fluorescent target was detected through $\sim 2.5 \mathrm{~cm}$ of human breast tissue and coregistered to the appropriate location on the tissue geometry. 


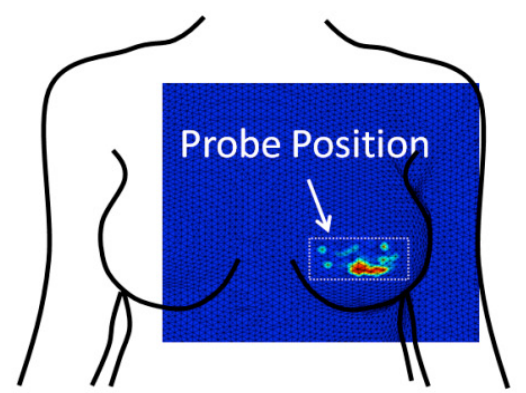

(A)

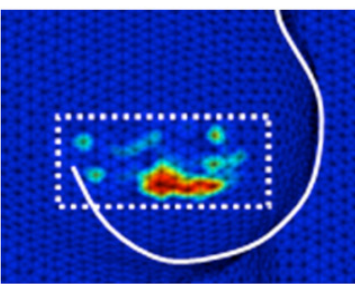

(C)

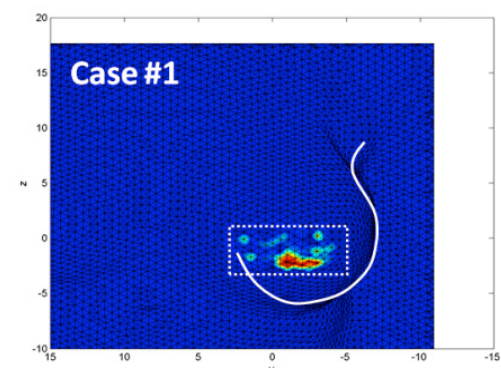

(B)

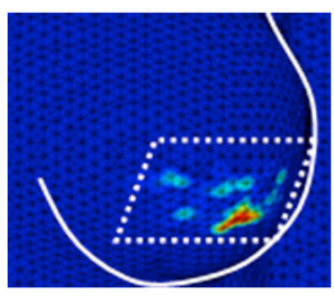

(D)

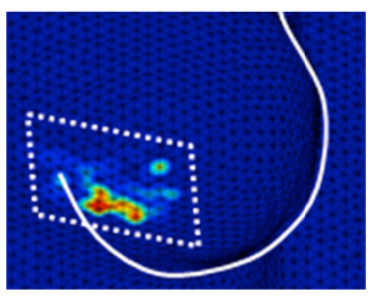

(E)

Figure 12.11 Coregistered images of fluorescence intensity data collected from a normal subject with an ICG-filled target using the initial set-up (subject seated in upright position). (A) Location of probe and image relative to the subject. (B) Case \#1 image: $0.45 \mathrm{~cm}^{3}$ target at the 6 o'clock position. (C) Zoomed image of Case \#1. (D) Case \#2 image: $0.45 \mathrm{~cm}^{3}$ target at the 4 o'clock position. (E) Case \#3 image: $0.23 \mathrm{~cm}^{3}$ target at the 8 o'clock position. All targets contain $1 \mu \mathrm{M}$ indocyanine green.

\subsubsection{Experimental Study II Automated Coregistered Imaging using the Modified Imaging Set-up}

Figure 12.12 shows automated coregistered images from a normal human subject using the modified imaging set-up with the acoustic tracker. The geometry was rotated to a $45^{\circ}$ angle over the $y$-axis in the coregistration software to correspond to the $45^{\circ}$ reclined position of the subject. The probe location was coregistered to the tissue at two different locations: the first location was centered over the breast tissue with the bottom of the probe positioned $2 \mathrm{~cm}$ beneath the nipple region (Figure 12.12A-C) and the second location was above the breast in the chest wall region with the bottom of the probe positioned $3 \mathrm{~cm}$ above the nipple region (Figure 12.12D-F). 


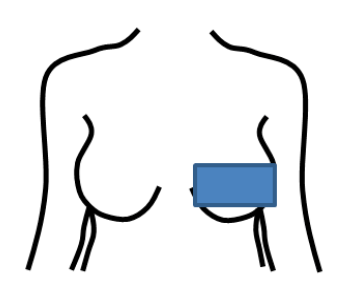

Probe Position \#1

(A)

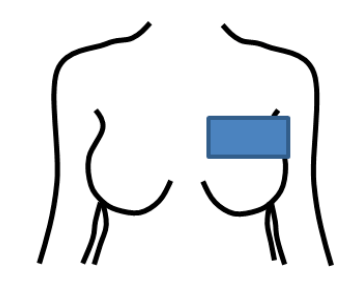

Probe Position \#2

(D)

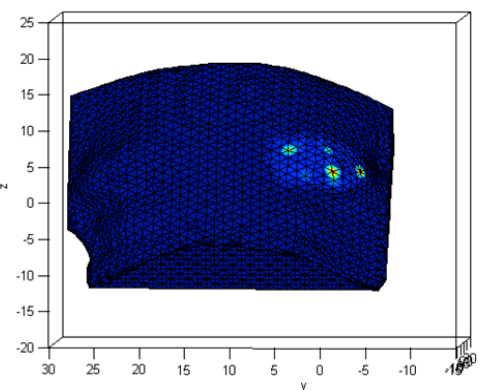

(B)

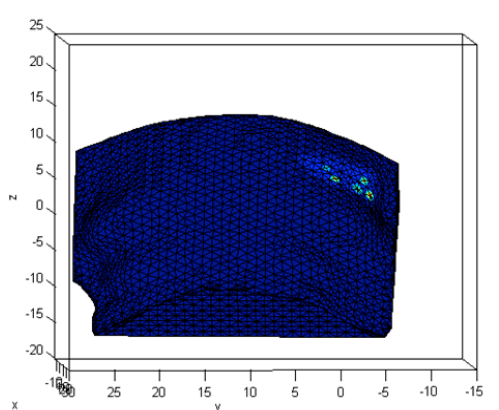

(E)

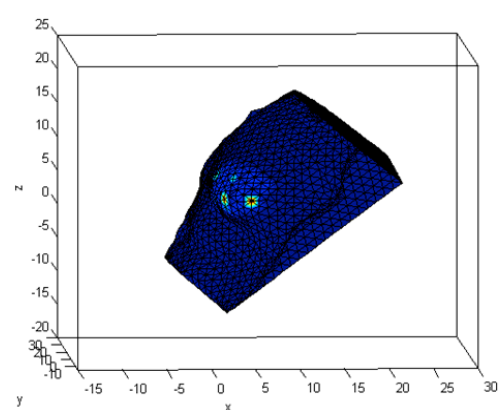

(C)

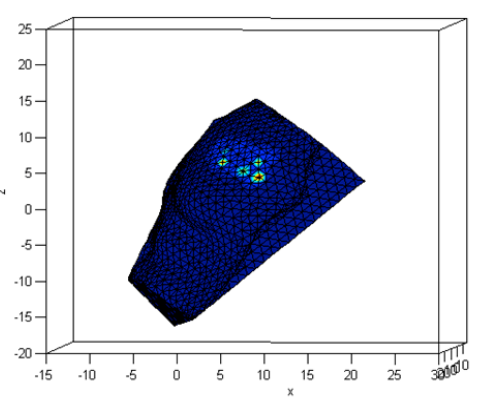

(F)

Figure 12.12 Automated coregistered images in a healthy subject (no target) using the modified imaging set-up. (A) Probe position \#1. (B) Frontal view of coregistered image at probe position \#1. (C) Side view of coregistered image at probe position \#1. (D) Probe position \#2. (E) Frontal view of coregistered image at probe position \#2. (F) Side view of coregistered image at probe position $\# 2$.

\subsubsection{Experimental Study III Manual Coregistered Imaging using the Modified Imaging Set-up}

Figure 12.13 shows results from absorption-based studies in a normal subject with a 0.45 $\mathrm{cm}^{3}$ target filled with $0.08 \%$ India ink located at the 6 o' clock position. Figure $12.13 \mathrm{~A}$ shows the location of the probe relative to the subject. Figure 12.13B shows the probe location placed manually in the post-process coregistration software, and Figure 12.13C shows the resulting coregistered image. It can be seen that the target is detected as a lower intensity (blue) signal indicating higher absorption at the location of the target. A 
background image was also collected (without target) and subtracted to eliminate background noise.

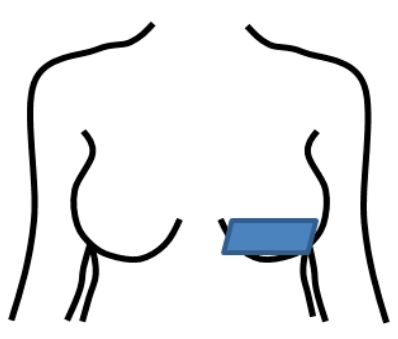

(A)

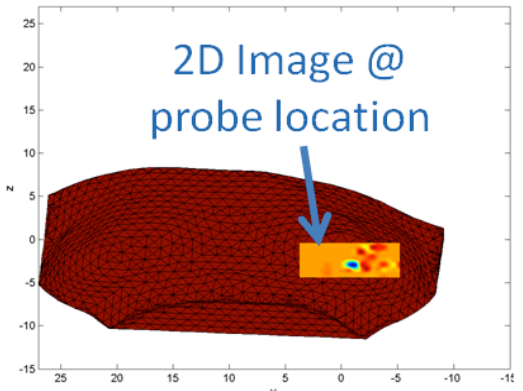

(B)

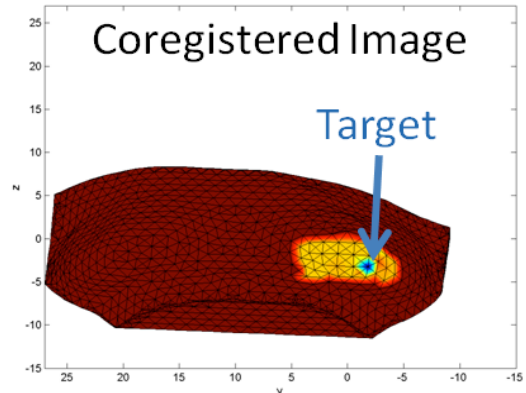

(C)

Figure 12.13 Manually coregistered images from a healthy subject with $0.45 \mathrm{~cm}^{3}$ target with $0.08 \%$ India ink placed under the breast tissue at the 6 o'clock position.

Figure 12.14 shows the results for the case of the lower concentration target $(0.02 \%$ India ink) placed at the 6 o' clock position. Figure 12.14A shows the location of the probe relative to the subject. Figure 12.14B shows the probe location placed manually in the post-process coregistration software, and Figure $12.14 \mathrm{C}$ shows the resulting coregistered image. The resulting contrast is lower due to the lower concentration of the absorbing agent, but the lower signal (blue) from the target can be seen at the target location in the $2 \mathrm{D}$ image (Figure 12.14B) and the coregistered image (Figure 12.14C). 

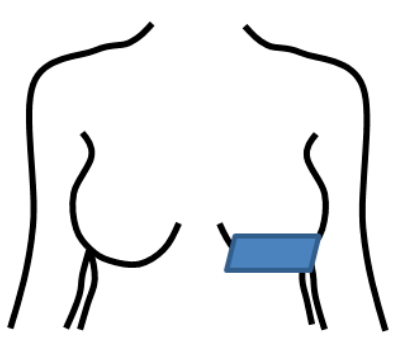

(A)

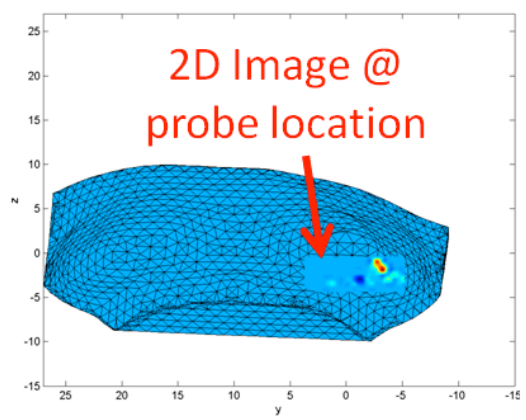

(B)

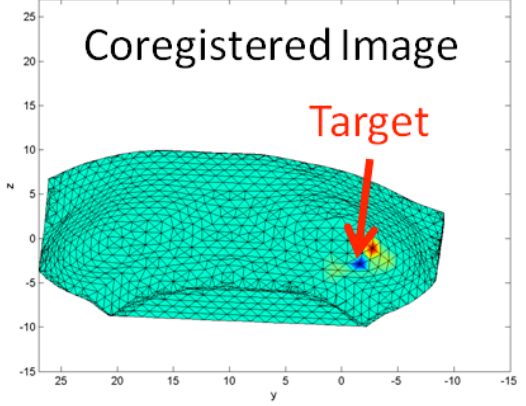

(C)

Figure 12.14 Manually coregistered images from a healthy subject with $0.45 \mathrm{~cm}^{3}$ target with $0.02 \%$ India ink placed under the breast tissue at the 6 o'clock position.

\subsection{DISCUSSION}

The modified imaging set-up was designed to overcome the limitations of the initial setup and improve the accuracy of automated coregistered imaging using the acoustic tracker. However, during implementation of the modified set-up, there were some difficulties encountered. The acoustic based tracker operates most effectively when the transmitter is placed level with the floor and the receiver is parallel to it. When the subject was reclined at a $45^{\circ}$ angle, the tracker lost line of sight on many areas of the tissue. To overcome this issue, the transmitter was mounted at a $45^{\circ}$ angle above the subject's head to enable it to be parallel with the receiver on the probe as it was placed on the breast tissue. However, with this set-up, the tracker would lose line of sight when the receiver was blocked by the subject's head. In addition, the tracker experienced significant fluctuations and losing/gaining of line-of-sight even when the probe was held at a constant location. This demonstrates that the accuracy of the tracker is poor even apart from any error on the part of the operator or movement of the subject or tissue. 
Hence, it was determined that an alternate method of automated tracking must be pursued. We are currently developing an optical based automated tracking method that will have much greater accuracy than the acoustic tracker (described in Chapter 16). Once the alternate tracking method is implemented, quantitative studies will be performed to determine the improvement in the accuracy of coregistered imaging in human subjects due to improvements in the imaging set-up and stability of the tracker.

Images can be manually coregistered to the tissue geometry if the probe location is accurately measured relative to the tissue. The MATLAB software (developed inhouse) that was used to generate the discretized mesh of the scanned geometry was modified to automatically find the initial reference point (at the nipple region) and lock it to the origin in the coregsitration software. The imaging locations were measured in centimeters and marked on the tissue for accurate positioning of the probe with reference to the origin (nipple). The images were collected with the hand-held imager at the predetermined locations and manually coregistered using post-process coregistration software. This method is valid as long as the tissue is relatively flat against the chest wall making the imaging surface close to planar. For tissues with greater curvature, it would be more difficult to manually coregister with accuracy due to rotation of the probe with respect to the origin. For efficient real-time tracking and imaging, a more accurate tracking system will be implemented. 


\subsection{SUMMARY AND CONCLUSION}

An automated coregistered imaging approach was implemented and validated in vivo on human breast tissue. A 3D scanner was used to acquire the breast tissue geometry from each individual subject and an acoustic based tracking system was used to track the probe location in real-time. The initial imaging set-up had several limitations which led to inaccuracy in the coregistered imaging. The set-up was modified to overcome these limitations; however, the tracking system was unstable. Results demonstrate the ability to detect an absorbing target through breast tissue of a healthy human subject and images were manually coregistered at the measured probe locations. These studies demonstrate that manual coregistered imaging can be performed in human subjects under simple conditions and automated coregistered imaging is feasible. The coregistered images will be used to perform 3D tomography in human breast tissue as described in the following chapter. 


\section{CHAPTER 13}

\section{Study VI: 3D Tomography in vivo with a Healthy Human Subject}

\subsection{INTRODUCTION}

Previous tomography studies performed using our hand-held optical imager involved phantoms with simple geometries and structured 3D mesh, and the experimental image data was manually coregistered at the known probe location with respect to the phantom. The major challenge in performing 3D tomography in human tissue as opposed to simple phantoms is to generate the discretized volume geometry (or mesh) for the human breast tissue. For simple cubicle phantoms, the geometry and mesh were generated within a single software (Gambit) and the same mesh was used for each phantom reconstruction. In the case of human subject studies, the various breast tissue geometries must be acquired for each subject and discretized into tetrahedral volume mesh. This chapter describes the procedure developed herein for acquiring a tetrahedral volume mesh of the various breast tissues of different human subjects and extracting the experimental information corresponding to each coregistered data set, which is needed to input into the reconstruction algorithm to perform 3D reconstructions. 3D fluorescence tomography is performed using a coregistered image from a healthy human subject with a superficially placed target. The results presented herein demonstrate for the first time the feasibility of performing 3D tomography in human breast tissue in vivo using a hand-held optical imager.

\subsection{MATERIALS AND METHODS}

\subsubsection{Procedure for 3D Mesh Acquisition and Reconstruction In Vivo}

A commercially available 3D surface scanner was employed to scan the breast tissue of each subject. The subject was seated upright in a 90 degree position and the motorized system 
was used to scan both sides of the breast tissue, chestwall, and ribcage. The resulting surface scan is shown in Figure 13.1 (left). The scanner software was used to cut the surface geometry to extract only the left breast in order to reduce computation time when performing the reconstruction.

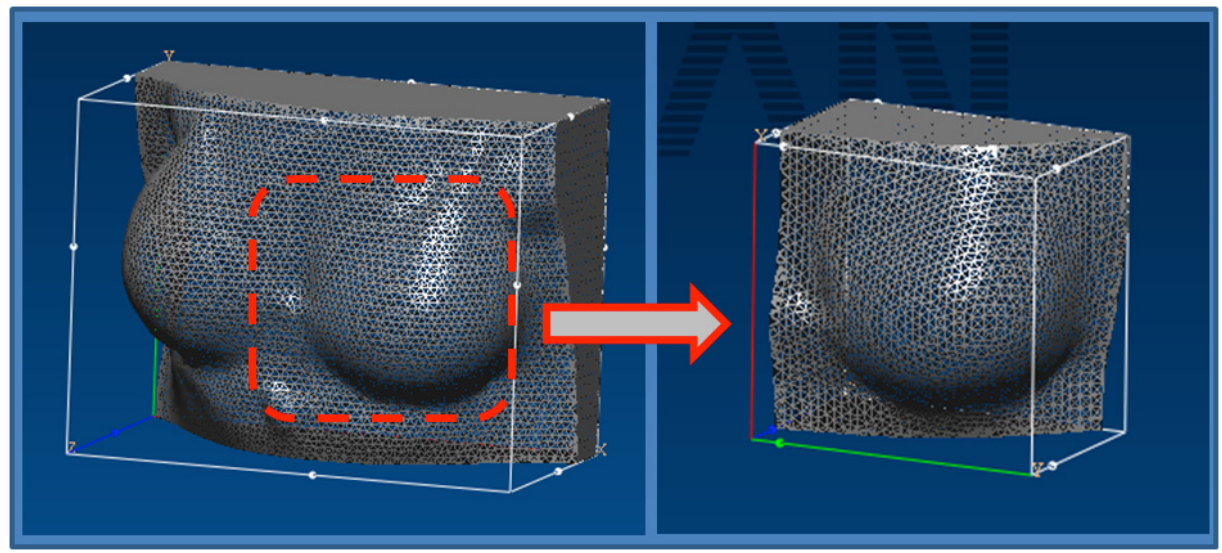

Figure 13.1 Scanned surface geometry of breast tissue. The imaged section was cut from the full geometry and used in the reconstruction.

The acquired surface scan was then imported into a software (GiD) and a volume was generated from the surface scan. Meshing softwares are typically designed such that a geometry is both generated and meshed within that same software. However, our application presented a unique complication in that we required an externally acquired complex geometry to be imported into the software and used to generate a $3 \mathrm{D}$ volume and mesh. After many trials and much troubleshooting it was determined that the method to acquire the mesh was to import the scanned surface into GiD and use the software to generate a volume. The volume generated in the GiD software is shown in Figure 13.2. The generated volume was then imported into Gambit which was used to generate the tetrahedral volume mesh. 


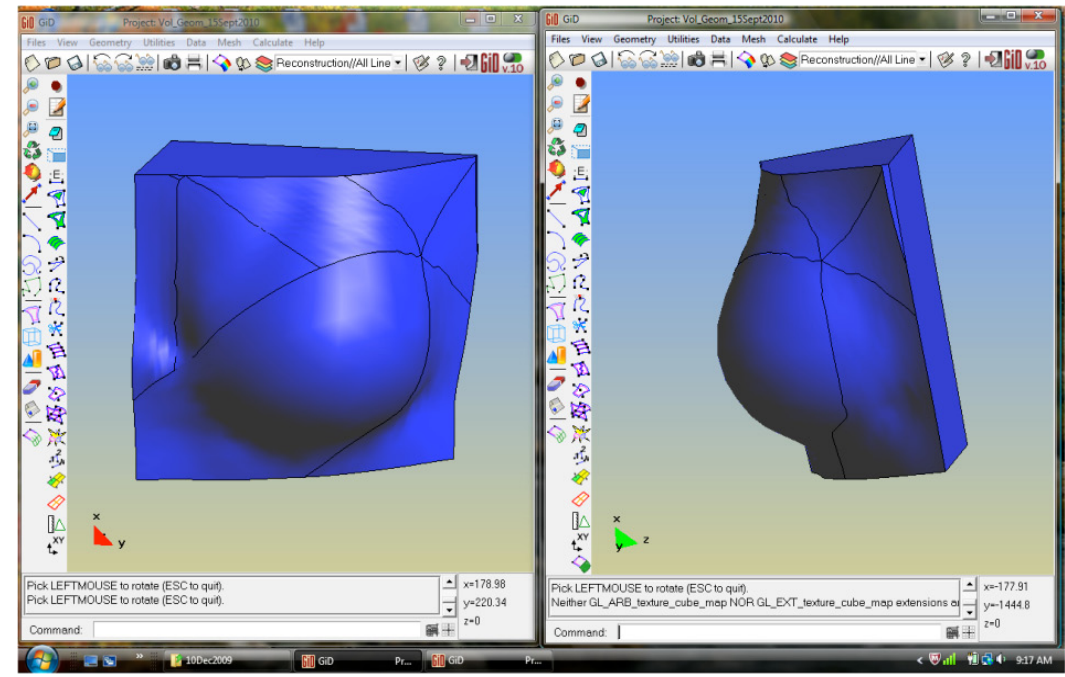

Figure 13.2 Volume geometry of scanned breast tissue.

After acquiring the tetrahedral volume mesh from Gambit, there were several steps required to prepare the mesh for importing into the reconstruction algorithm. The first step was to determine the coordinates of the optical data image (collected by the hand-held device) with respect to the volume mesh (since the original coregistered image used only a surface mesh to reduce the imaging time and the coordinates are different in the volume mesh generated for reconstruction). A post-processing version of the coregistered imaging software was used to coregister the image to the new volume mesh using the positional information stored from the original surface coregistered image acquired during live imaging. A code was developed in MATLAB to then extract the coordinates and intensity data for use in the reconstruction algorithm.

Another piece needed for the reconstruction algorithm was the coordinates of the sources and detectors relative to the volume mesh. In the case of cubical phantoms, the 
coordinates on the simple geometry were manually entered since the $0.5 \mathrm{~cm}$ spaced grid of detectors aligned perfectly with the $0.25 \mathrm{~cm}$ spaced grid of the cubical mesh. In the case of human breast tissue, the mesh is unstructured and the probe location is not perfectly aligned with the mesh coordinates. Hence, a method was developed to determine the source and detector coordinates of any random probe position on the various unstructured volume meshes. A grid was generated corresponding to the source and detector locations of the hand-held probe image. The source locations in the grid were assigned a high value and the detector locations were assigned a low value. The grid was then coregistered to the volume mesh using the same positional information from the original surface coregistered image. A code was developed in MATLAB to extract the coordinate locations of the sources and detectors from the volume coregistered image according to the relative intensity values.

Since at this point the coordinates now correspond to the coordinate system in the coregistration software (which is different from the original coordinate system assigned by the Gambit software), the volume mesh from the coregistration software was exported to be used in the reconstruction algorithm. However, a complication existed in the mesh exported from the coregistration software. The coregistration software assigned nodes and elements of the mesh using a different method than the Gambit software. In the Gambit software, the nodes are assigned first and then connected to form the elements, resulting in more elements than nodes. The coregistration software (using the MATLAB 'patch' function) first generates the elements and then assigns the node numbers individually within each element (Figure 13.3). Hence, adjacent elements assign multiple numbers to the same node. This resulted in a very large number of nodes compared to elements which greatly and 
unnecessarily increases the computational time, and the mesh was incompatible with the reconstruction algorithm.
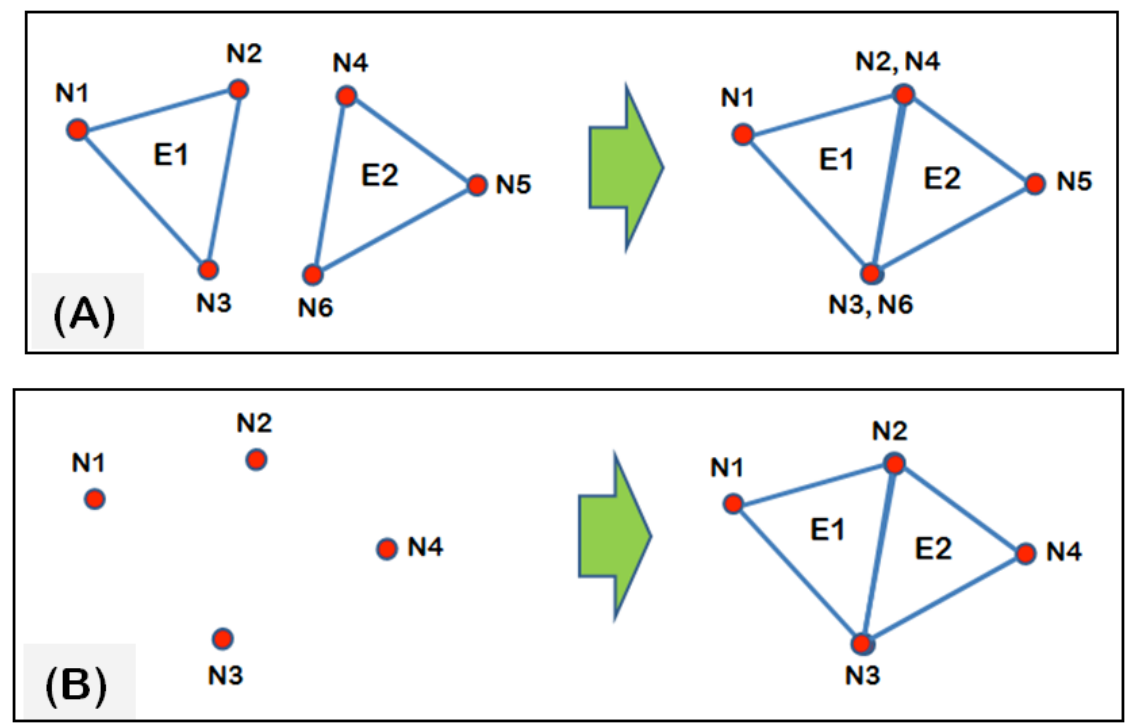

Figure 13.3 Illustration of the two methods of assigning node numbers. (A) The mesh generated by MATLAB and exported from the coregistration software assigns the elements first and then gives each node in the element number assignments independent of the adjacent elements resulting in multiple numbers assigned to the same node. (B) The preferred method is where the node numbers are assigned first and then elements are constructed among those nodes such that each node is shared by multiple elements and only assigned a single number resulting in much fewer nodes than elements.

To overcome this complication, a code was developed in MATLAB to eliminate the repeated nodes and reassign the node numbers within the elements correspondingly. Additionally, a code was generated in MATLAB to reassign the node numbers corresponding to the source and detector locations and the image intensity data according to the corrected numbers. The final mesh and all the extracted information were then imported into the reconstruction algorithm to perform 3D tomography.

The entire procedure developed herein for acquiring the tetrahedral volume mesh, extracting the experimental information, and performing 3D tomography in vivo in order to reconstruct 
a target within the breast tissue geometry of a human subject is outlined in the following ten major steps:

Step 1: Acquire the coregistered image of optical intensity data from the breast tissue (detailed in Chapter 12 above).

Step 2: Use the 3D surface geometry from the Fastscan to generate a 3D volume. The 3D surface geometry that was scanned from the breast tissue was imported in IGES format into the GiD software which was used to generate a $3 \mathrm{D}$ volume from the surface. Figure 13.1 shows the volume geometry generated in the GiD software.

Step 3: Generate a 3D tetrahedral volume mesh and corresponding triangular surface (or boundary) mesh for the volume breast geometry. The $3 \mathrm{D}$ volume from the GiD software was loaded into Gambit software which was used to generate the unstructured triangular and tetrahedral mesh.

Step 4: Adjust the coordinates of the Gambit mesh to correspond to the coregistered image. The coordinate system of the mesh generated in Gambit is independent of the coordinate system of the coregistered image. The Gambit mesh was imported into the postprocess coregistration software and locked to the location of the geometry during imaging. The positional information preserved in the coregistered data that was acquired during live imaging was used to coregister the image at the same location in the Gambit mesh.

Step 5: Determine the nodes and coordinates of the breast mesh that correspond to the source and detector positions in the coregistered probe location. The positional information of the coregistered probe location was used to find the closest nodes in the unstructured breast mesh to each source and detector in the probe, and the coordinates of 
those nodes were assigned as the coordinates of the sources and detectors. The procedure developed for performing this step ensures that the source and detector positions assigned to the mesh correspond to the data collected with the probe and coregistered to the tissue during live imaging.

Step 6: Eliminate duplicate nodes from the breast mesh (as exported from the coregistration software) and assign the adjusted node numbers appropriately to each element.

\section{Step 7: Convert text files and source/detector positional information into data files} that will be used in the reconstruction code. This step must be performed for each new mesh geometry and each different probe position within the same geometry.

Step 8: Acquire the intensity data for each detector position in the 3D breast mesh from the coregistered image data. A code was written in MATLAB to extract the intensity data from the coregistered mesh at the detector nodes located in Step 5 and 6 and generate a data file containing the coordinates of the detector nodes and the corresponding intensity values from the breast data.

Step 9: Use the intensity data acquired from Step 8 to generate the data files used in the AEKF algorithm. These data files contain the AC, DC, phase, and modulation depth information (or DC only for CW data) along with the optical properties of the background tissue. In this case, average optical property values of human breast tissue acquired from the literature [134] were used in the reconstruction. 
Step 10: Use the AEKF based algorithm to reconstruct the fluorescent/absorption intensity values within the $3 \mathrm{D}$ discretized geometry of the breast tissue. The AEKF algorithm is detailed in Chapter 7.

\subsubsection{Fluorescence Tomography in a Healthy Human Subject}

A 3D reconstruction was performed using data acquired from a healthy human subject with a $0.45 \mathrm{~cm}^{3}$ spherical target containing $1 \mu \mathrm{M}$ ICG placed underneath the flap of the breast tissue at the 6 o'clock position (Case \#1 in Section 12.3). The data was acquired by automated self-coregistered imaging with the acoustic tracker using the initial imaging set-up where the subject sat in a vertical upright position and the geometry was acquired by the motorized 3D scanning technique. The geometry consisted of both sides of the breast tissue and part of the chest wall and ribcage. In order to reduce computational time, the geometry was cut to include only the breast tissue region in the vicinity where the data was acquired with the optical imager (as described in Section 13.2.1). The reduced geometry was manually placed at the appropriate location within the coordinate system using the post-process coregistration software. The ten steps described in Section 13.2.1 were executed in order to acquire the 3D meshed geometry and perform 3D tomography in human breast tissue. The initial p-value in the AEKF reconstruction algorithm was set to 0.01. The input optical property values were $0.05 \mathrm{~cm}^{-1}$ for $\mu_{\mathrm{ax}}$ and $\mu_{\mathrm{am}}$, and $8.0 \mathrm{~cm}^{-1}$ for $\mu_{\mathrm{sx}}{ }^{\prime}$ and $\mu_{\mathrm{sm}}$ ' (average values of breast tissue optical properties obtained from the literature [134]. The initial guess for the optical property distribution (in this case, $\mu_{\text {axf }}$ ) was uniformly set to $0.003 \mathrm{~cm}^{-1}$. The modulation frequency $(\square)$ was set to zero for $\mathrm{CW}$ based reconstruction and only the real part of the equations were used. 


\subsection{RESULTS}

The experimental data used for the reconstruction is shown in Figure 13.4, where the yellow dashed line shows the probe location with respect to the disctretized tissue geometry. The fluorescent signal from the target was detected close to the target location (6 o'clock position).

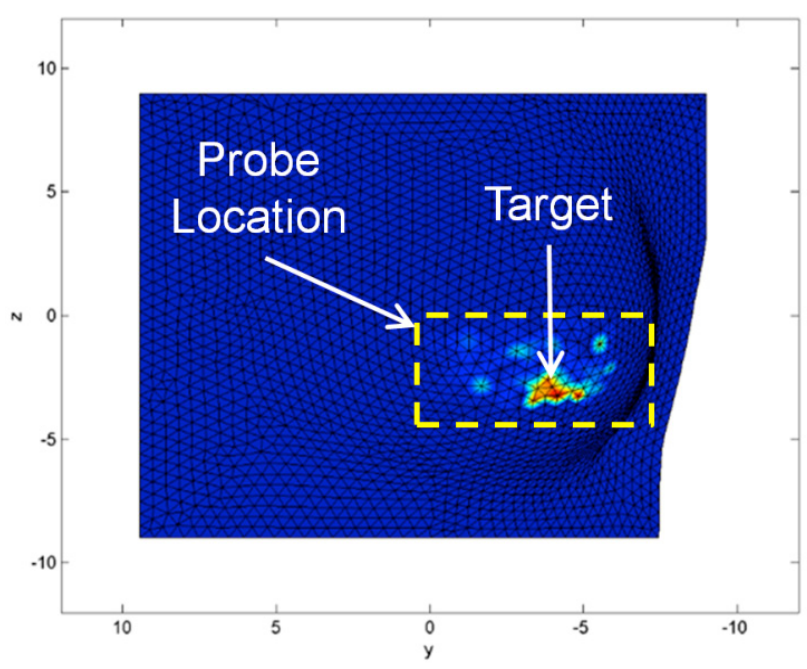

Figure 13.4: Automated coregistered image of a fluorescent target placed beneath the breast tissue in a normal subject which was used to perform the $3 \mathrm{D}$ reconstruction.

Figure 13.5 shows the experimental result of the reconstruction plotted in Tecplot. Figure $13.5 \mathrm{~A}$ shows the $3 \mathrm{D}$ tetrahedral unstructured mesh of the breast tissue geometry and Figure 13.5B shows the recovered fluorescence intensity as a series of slices in the $\mathrm{x}$-plane. The slices are plotted in profile view to show their location within the tissue geometry. Individual detailed slices are shown in frontal view in Figure 13.6. 

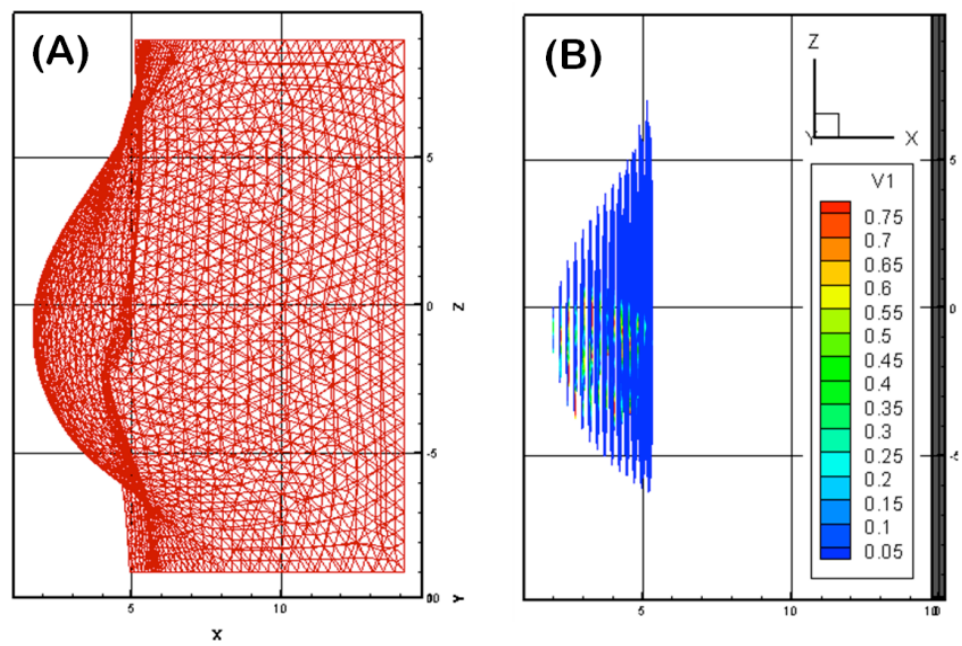

Figure 13.5: (A) Plot of the 3D tetrahedral unstructured mesh used in the reconstruction. (B) Side view of contour slices in the $\mathrm{x}$-plane.

The coronal slices in Figure 13.6 begin at the tissue surface close to the central (nipple) region and progress depth-wise toward the chest wall. The initial slices at depths below 1.0 $\mathrm{cm}$ (slices 1-3) show signal from the $2 \mathrm{D}$ target location although the depth of the signal is less than the true target depth $(\sim 2.5 \mathrm{~cm})$. At a depth of $1.0 \mathrm{~cm}$ and greater (slices $4-12)$, additional signal appears to the left side of the breast tissue away from the target location. 


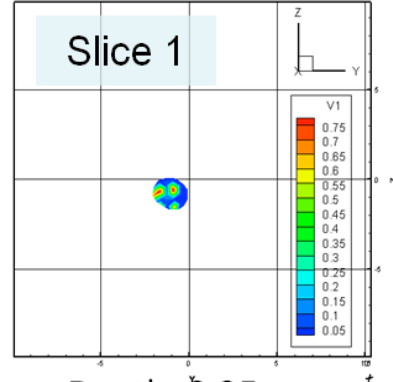

Depth $=0.25 \mathrm{~cm}$

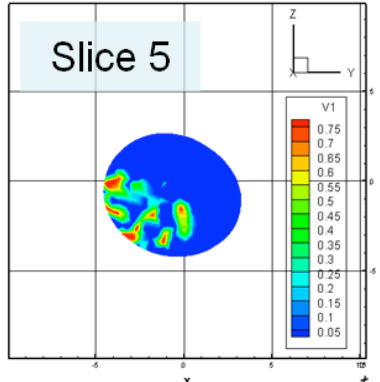

Depth $=1.25 \mathrm{~cm}$

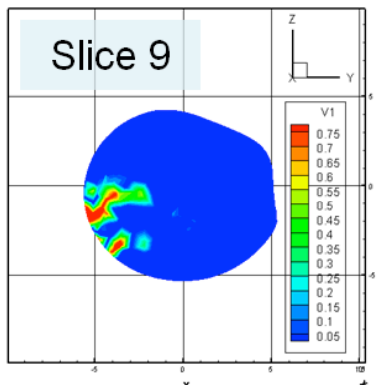

Depth=2.25 cm

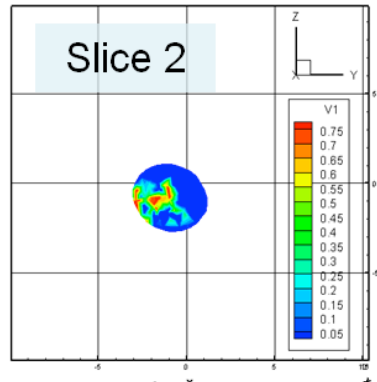

Depth $=0.5 \mathrm{~cm}$

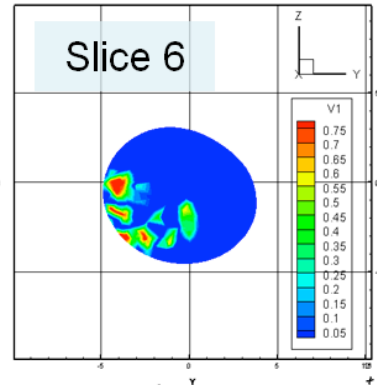

Depth $=1.5 \mathrm{~cm}$

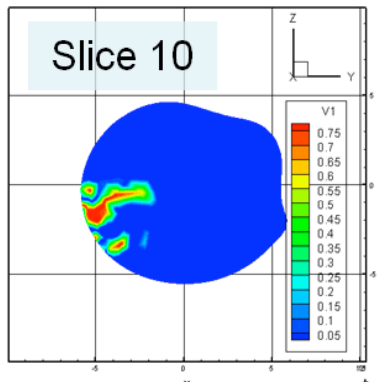

Depth $=2.5 \mathrm{~cm}$

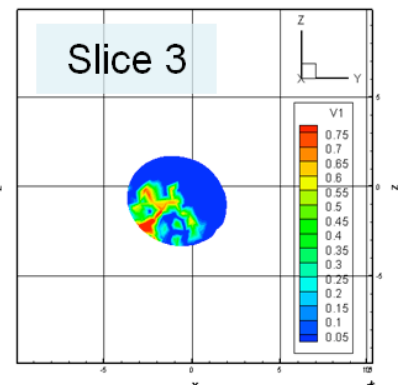

Depth $=0.75 \mathrm{~cm}$

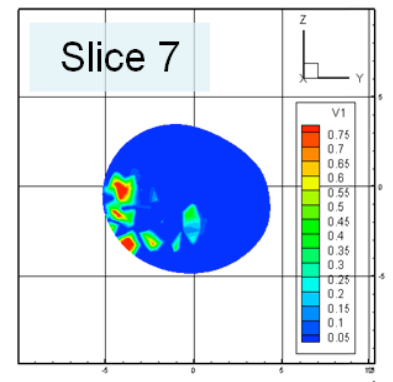

Depth $=1.75 \mathrm{~cm}$

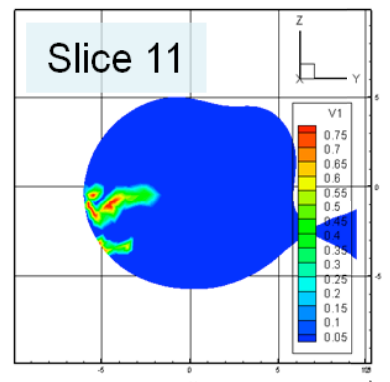

Depth $=2.75 \mathrm{~cm}$

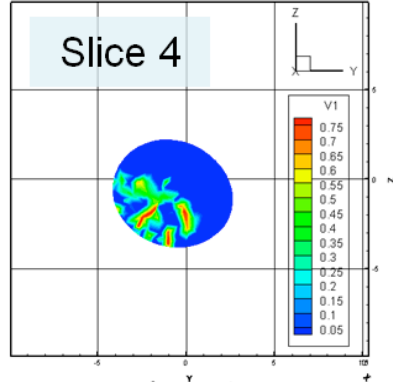

Depth $=1.0 \mathrm{~cm}$

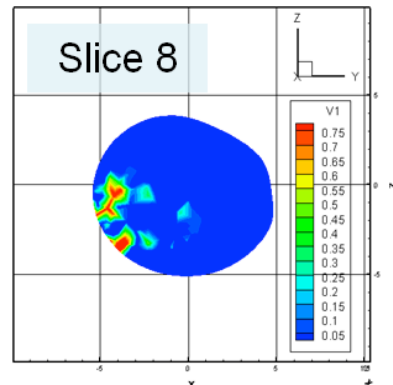

Depth $=2.0 \mathrm{~cm}$

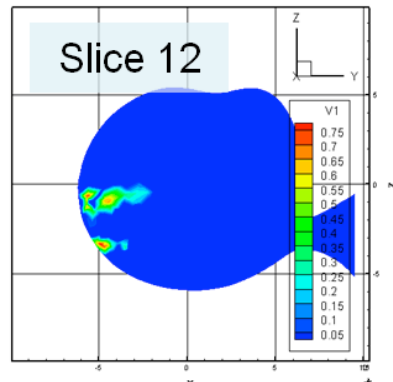

Depth $=3.0 \mathrm{~cm}$

Figure 13.6: Contour slices in the x-plane at $0.25 \mathrm{~cm}$ intervals. Slice depth is indicated as depth from the tissue surface at the center (nipple) region.

Figure 13.7 shows slices in $y$-plane representing the sagittal view. Slice 4 in the center gives the sagittal slice at the $y=0$ location which is the central part of the tissue. The other slices represent the locations in $0.25 \mathrm{~cm}$ increments to the left (slices 1-3) and right (slices 5-7) of the center. The images show that within this central region, there is a maximum signal intensity at the central location of the tissue which corresponds to the 6 
o'clock position of the fluorescent target, and the signal diminishes in either direction away from the center.

Slice 1

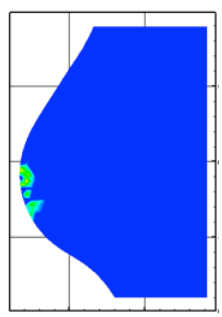

$y=-0.75$
Slice 2

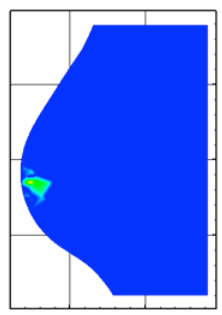

$y=-0.5$
Slice 3

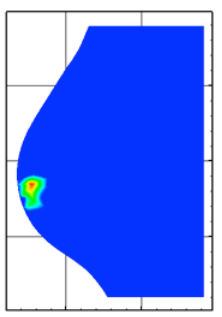

$y=-0.25$
Slice 4

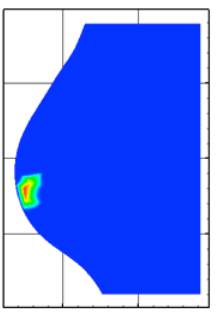

$y=0$
Slice 5

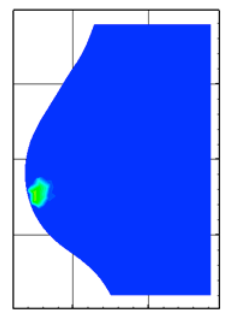

$y=0.25$
Slice 6

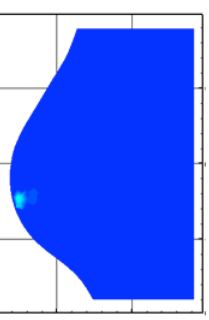

$y=0.5$
Slice 7

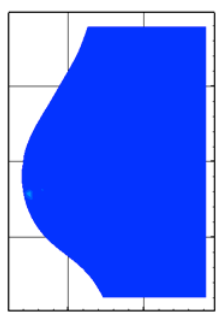

$y=0.75$

Figure 13.7 Contour slices in the y-plane at $0.25 \mathrm{~cm}$ intervals.

The quantitative results for the in vivo 3D reconstruction are provided in Table 13.1. The recovered $\mu_{\text {axf }}$ value (9.17) is much larger than expected, which is due to the presence of signals from artifacts which are all included in the total $\mu_{\text {axf }}$ value. The true target location was approximated at $(\mathrm{x}, \mathrm{y}, \mathrm{z})=(2.5,0,0)$ since the target was placed in the center underneath the flap of the tissue and the approximate tissue depth (x-dimension) was $2.5 \mathrm{~cm}$ (as measured by a Vernier caliper). The recovered target location was $(\mathrm{x}, \mathrm{y}, \mathrm{z})=(3 \cdot 0,-3 \cdot 3,-1.8)$ and the total distance off was $3.75 \mathrm{~cm}$. The distance off can be due to two factors: (i) inaccuracy in the estimation of the true target location, and (ii) the artifacts that appear toward the left side of the reconstruction (as shown in Figure 13.6) which can be mistaken as the true location due to the high intensity of the signal. Reduction in artifacts and better estimation of the true target location will likely improve the quantitative results. 
Table 13.1 Quantitative results for the in vivo 3D reconstruction: recovered $\mu_{\text {axf }}$, true target volume, recovered target volume, approximate true target location, recovered target location, total distance off.

\begin{tabular}{|c|c|}
\hline Recovered $\mu_{\text {axf }}$ & 9.17 \\
\hline True Target Volume $\left(\mathrm{cm}^{3}\right)$ & 0.45 \\
\hline $\begin{array}{c}\text { Recovered Target Volume } \\
\left(\mathrm{cm}^{3}\right)\end{array}$ & 15.0 \\
\hline $\begin{array}{c}\text { Approximate True Target } \\
\text { Location }(\mathrm{cm})\end{array}$ & $(\mathrm{x}, \mathrm{y}, \mathrm{z})=(2.5,0,0)$ \\
\hline $\begin{array}{c}\text { Recovered Target Location } \\
(\mathrm{cm})\end{array}$ & $(\mathrm{x}, \mathrm{y}, \mathrm{z})=(3.0,-3.3,-1.8)$ \\
\hline Total Distance Off $(\mathrm{cm})$ & 3.75 \\
\hline
\end{tabular}

\subsection{DISCUSSION}

Three-dimensional tomography of breast tissue in a human subject using a hand-held based optical imager was demonstrated for the first time. The reconstructed result shows recovered signal at the true target location but at a shallower depth than the true target location. This depth recovery limitation is a common phenomenon when reflectance based measurements are used. More accurate depth recovery may be achieved by using a combination of transilluminated and reflected measurements or by using multiple projections in reflectance mode.

Additional signal away from the target location was also present on the left side of the tissue. This artifact signal might be the result of poor contact with the tissue on one side of the probe causing detection of excitation light that was reflected off the tissue surface. The subtraction based post-processing technique was applied to eliminate excitation leakage; however, the probe might have shifted during removal of the target causing the background image to have a different noise distribution from the image with the target. 
For this initial preliminary tomography study, a coarse mesh was used in order to reduce computational time and effort while the reconstruction procedure was developed. Use of a finer mesh could produce a more accurate reconstruction result. In addition, a varied mesh might be used which is more refined close to the target location and more coarse away from the target location which would further reduce the noise away from the target. This would be applicable in a clinical setting since FDOT is not currently used for screening and hence the region of tissue suspected of containing a tumor would be known.

This study used data from a normal human subject with a fluorescent target placed noninvasively beneath the flap of breast tissue, between the breast tissue and the ribcage. Hence the light passed through two layers of skin and a full layer of breast tissue during both the incident and returning path which would produce a different result than an actual tumor embedded within the breast itself under a few centimeters of tissue.

This preliminary study demonstrates the feasibility of performing 3D tomography in human breast tissue using the ten step procedure developed herein. In the case presented here, the true target location was roughly estimated and the results analyzed qualitatively. Future work will involve extensive tomography studies in healthy subjects and breast cancer patients to improve the accuracy of the quantitative results.

\subsection{SUMMARY AND CONCLUSION}

A procedure for performing 3D tomography in human tissue using automated selfcoregistered experimental data was developed. A preliminary reconstruction study was performed using the self-coregistered data from a normal human subject with a fluorescent

target placed underneath the breast tissue. The result shows fluorescent intensity signal reconstruction close to the true target location along with artifact signal away from the target 
location. This study demonstrates the feasibility of performing 3D tomographic imaging in human breast tissue using a hand-held based optical imaging system. 


\section{CHAPTER 14 \\ Study VII: In vivo Imaging of Breast Cancer Subjects}

\subsection{INTRODUCTION}

The studies described in the previous chapters involved preclinical testing of a hand-held optical imaging system for breast cancer diagnosis. The final stage of this dissertation involves testing the performance of the device in actual breast cancer subjects. This chapter describes preliminary studies designed to demonstrate the feasibility of imaging breast tumors in human subjects using our hand-held optical imager.

\subsection{MATERIALS AND METHODS}

Diffuse optical imaging studies were performed with two subjects (age 64 and 51) with breast cancers masses confirmed by prior image modalities (i.e. x-ray mammography, ultrasound, MRI). The studies were approved by the FIU Institutional Review Board and the subjects signed a consent form prior to the study and a HIPAA authorization form (for release of the medical records). For the diffuse optical studies (non-fluorescent) one neutral density filter (OD 4) was used to uniformly attenuate the collected laser light. Continuous wave based images were collected (average of 5 frames) using an exposure time of $200 \mathrm{msec}$. The gain on the image intensifier was set while the probe was placed at the initial probe location and held constant during the entire study performed at various probe locations.

The studies were performed using the modified imaging set-up described in Chapter 12 , where the subject lay in the supine position in a recliner chair at a $45^{\circ}$ angle. The probe was placed at different locations on the ipsilateral (tumor-containing) and contralateral (nontumor-containing) breast and images of continuous-wave absorption-based measurements were collected. The probe positions were determined visually to cover the entire breast 
tissue. Initially the probe was centered over the 12 o'clock position covering the upper region of the breast tissue and part of the chest wall (probe location \#1 in Figure 14.1), then moved vertically down toward the 6 o'clock position (probe location \#4 in Figure 14.1). The four imaging locations relative to the subject are shown in Figure 14.1 (not to scale).

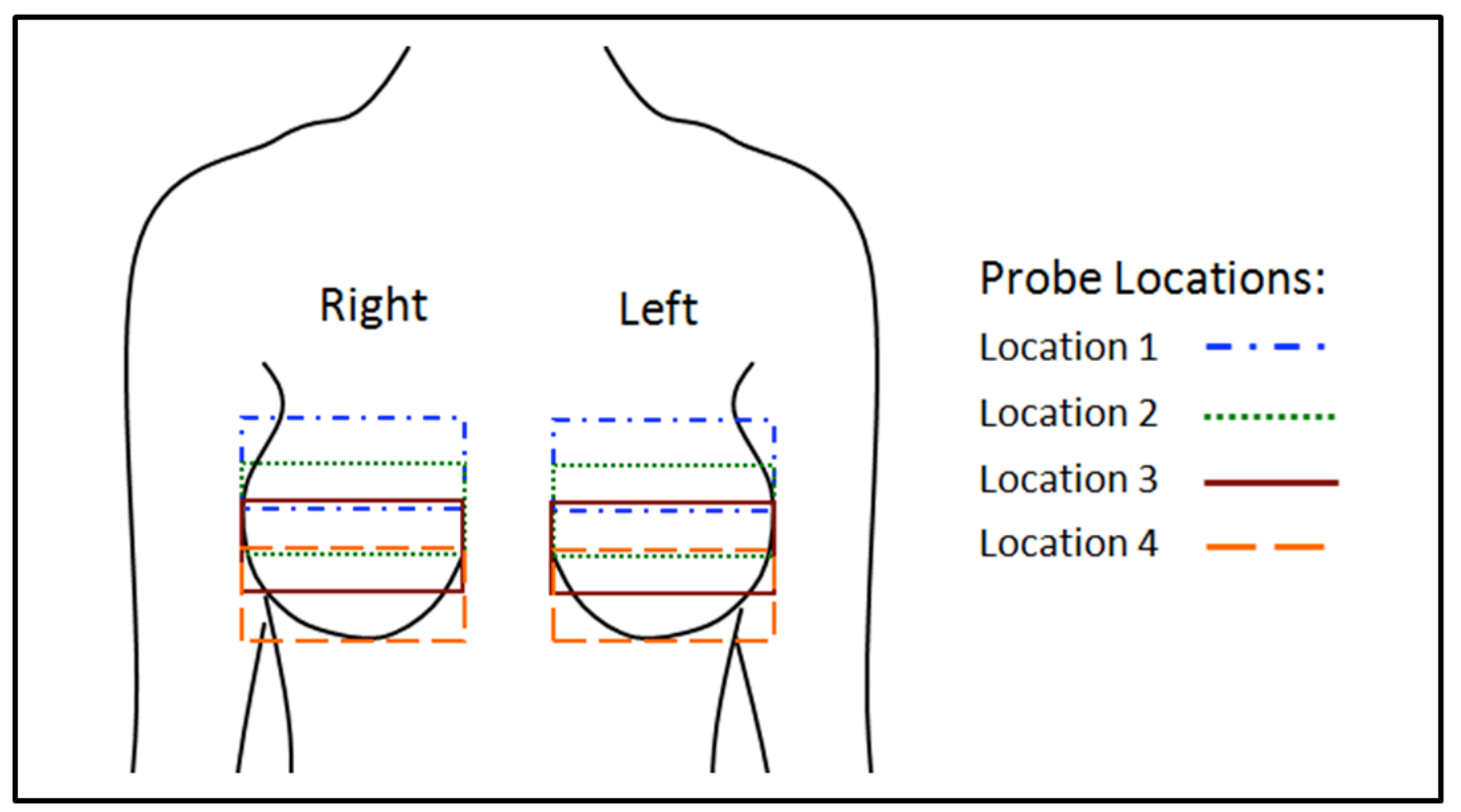

Figure 14.1: Schematic showing the probe locations during the imaging studies. The probe was placed at four different locations on the left breast tissue followed by the same four locations on the right breast tissue. All probe positions were estimated with reference to the nipple location and are not drawn to scale in the schematic.

Currently, a more structured and accurate approach is developed for measuring and marking the probe locations using the same method in all subjects (described in detail in Section 11.2 and Figure 11.2). For the studies describe herein, the positions were estimated at the following locations corresponding to Figure 11.2: UM4 (location 1), UM2 (location 2), LM2 (location 3), and LM4 (location 4). In each case, both the left and right breast tissues were imaged at the same symmetrical probe locations and the images from the contralateral breast were normalized and used in subtraction based post-processing. In every case, the 
normalized intensity data from the ipsilateral breast was subtracted from the normalized intensity data from the contralateral breast so that the signal due to higher absorption shows as a higher (more red) signal in the $2 \mathrm{D}$ contour plot.

For subject \#1 a second set of images were collected from the outer side of the breast tissue under the arm because the medical records indicated lymphatic spread to the axillary lymph nodes. The subject was asked to lie on the right side while resting the left arm above the head and the probe was placed under the arm for location \#1. The probe was then moved down toward the ribcage and images were collected at four different locations as shown in Figure 14.2. The axillary lymph nodes are located in the outer chest wall region under the arm as indicated in Figure 14.2. The entire set of images was repeated.

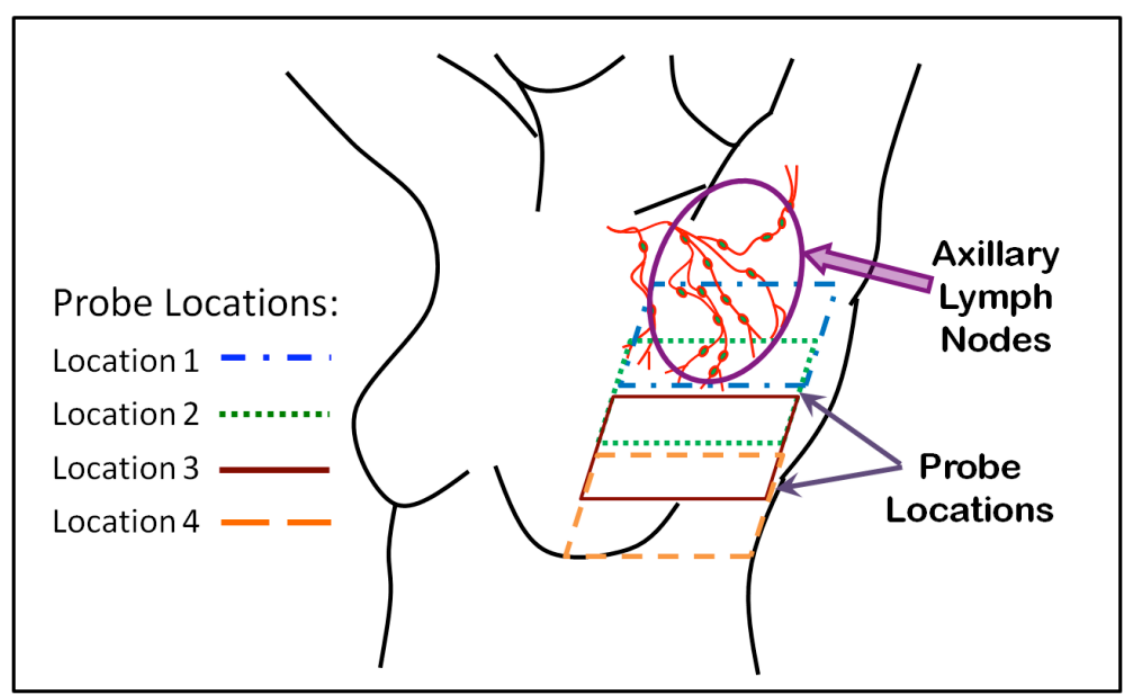

Figure 14.2 Probe locations for second set of images in subject \#2. The subject was asked to lie on the right side and rest the left arm above the head while the probe was placed at four different locations on the outer side of the left breast starting under the arm and moving down toward the ribcage. The procedure was repeated on the right breast at symmetric locations. All probe positions were estimated with reference to the nipple location and are not drawn to scale in the schematic. 


\subsection{RESULTS}

The optical images from both subjects were compared to the medical records provided. For subject \#1, a palpable mass in the left breast produced a negative result in $\mathrm{x}$-ray mammography. However, MRI images showed a region of abnormal tissue behind the nipple in the left breast which was determined by needle biopsy to be invasive ductal carcinoma with lymphatic spread. Figure 14.3 shows slices from an MRI scan. The yellow arrows indicate the abnormal tissue located in the periariolar region (i.e. near the nipple area).
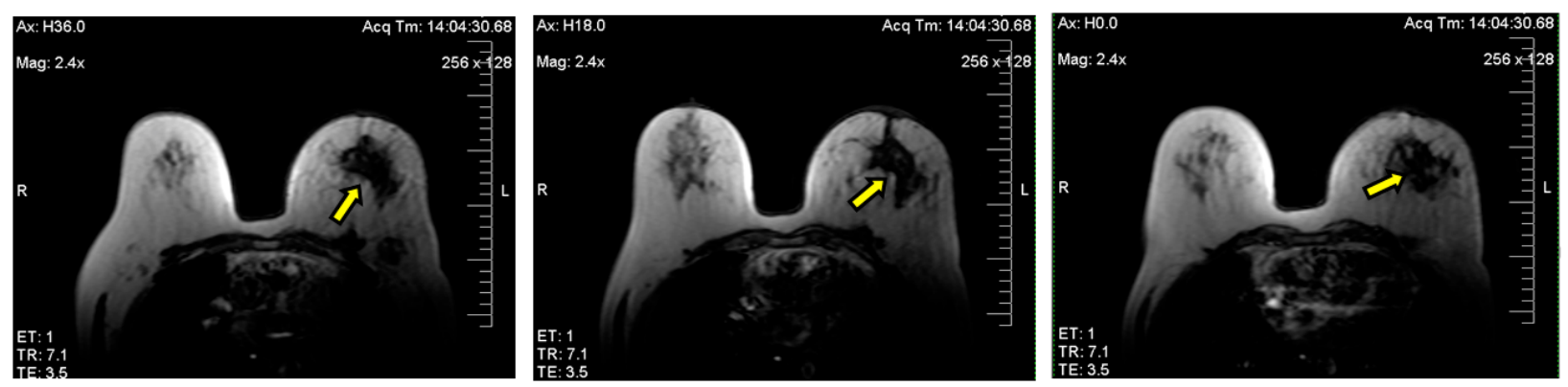

Figure 14.3: Three slices of an MRI scan from 64 year-old breast cancer patient. The yellow arrows indicate the location of abnormal tissue behind the nipple region.

Figure 14.4 shows the first set of continuous-wave absorption-based images collected from subject \#1 using the hand-held optical imager (repetition \#1). For each of the four probe locations (indicated in Figure 14.1), an image was collected from the left breast (on left) and right breast (center) and the data were subtracted to yield the post-processed images (on right). The optical signal due to absorption is indicated by the dashed yellow line. The location of the signal corresponds to the location of abnormal tissue indicated in the MRI images (Figure 14.3). The probe locations began at the top part of the breast and moved down toward the bottom of the breast tissue (as described in the previous section). Since 
the region of abnormal tissue was located in the central part of the breast tissue behind the nipple, the optical signal from that region of tissue would be visible in the lower part of the image for probe position \#1, in the center of image for probe positions \#2 and \#3, and toward the top of the image for probe position \#4. These areas are indicated in the images by a yellow dotted circle. Additional signal appears in the lower right corner of each postprocessed image which is likely due to incomplete contact of the outer edge of probe with the tissue. Figure 14.5 shows the results from the second repetition of images collected at the same locations and post-processed. The images show similar result to Figure 14.4 which demonstrates repeatability of the data collected.

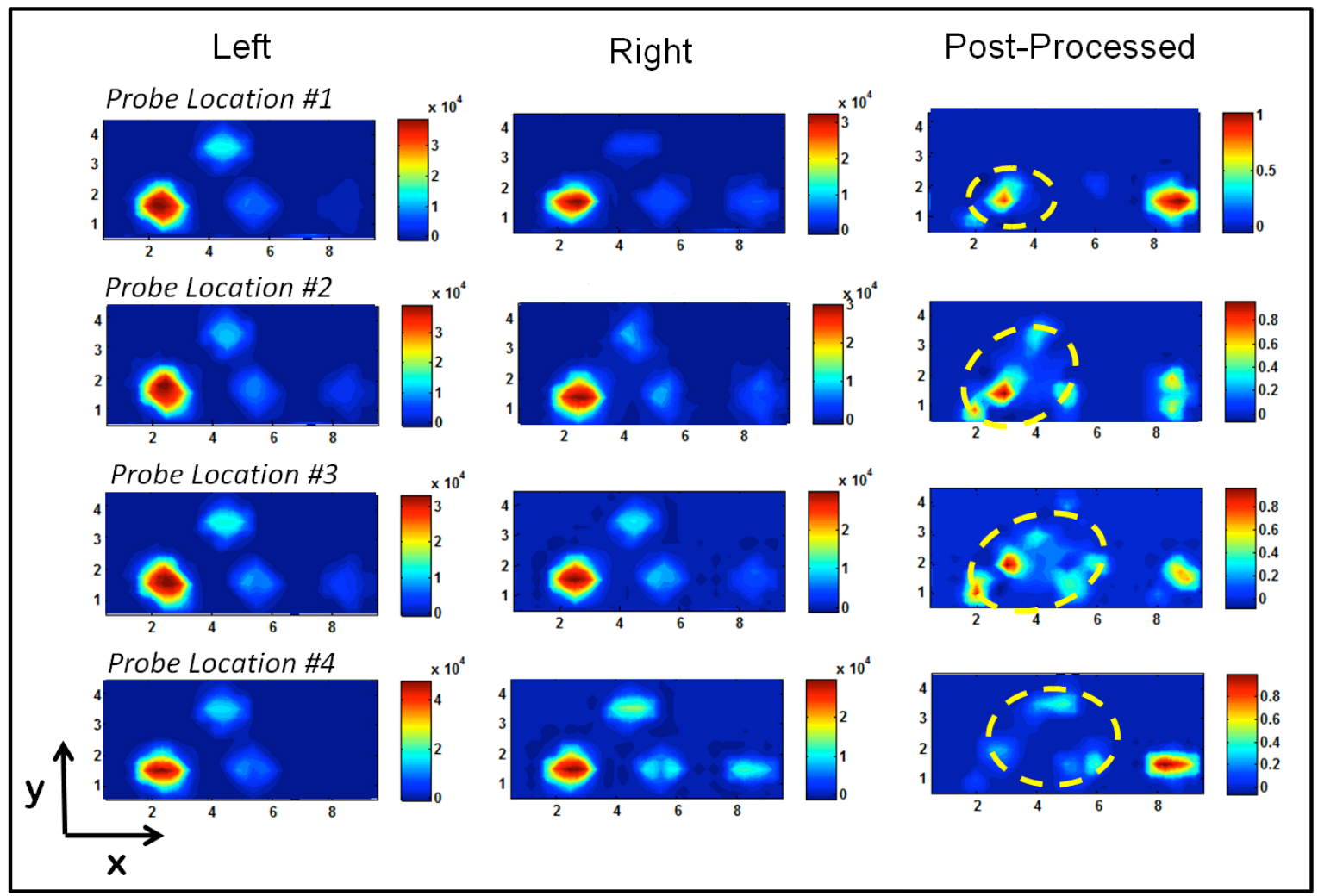

Figure 14.4: Continuous-wave absorption-based optical images collected from subject \#1 at four probe locations (first repetition of images). The images on the left were collected from the left breast tissue and the images in the center were collected from the right breast tissue. The post-processed images on the right represent the normalized intensity data from the ipsilateral (left) breast subtracted from the normalized intensity data from the contralateral (right) breast showing the optical signal due 
to absorption as a higher (red) signal in the images. The yellow dotted circle was hand-drawn to represent the region of interest in each image.

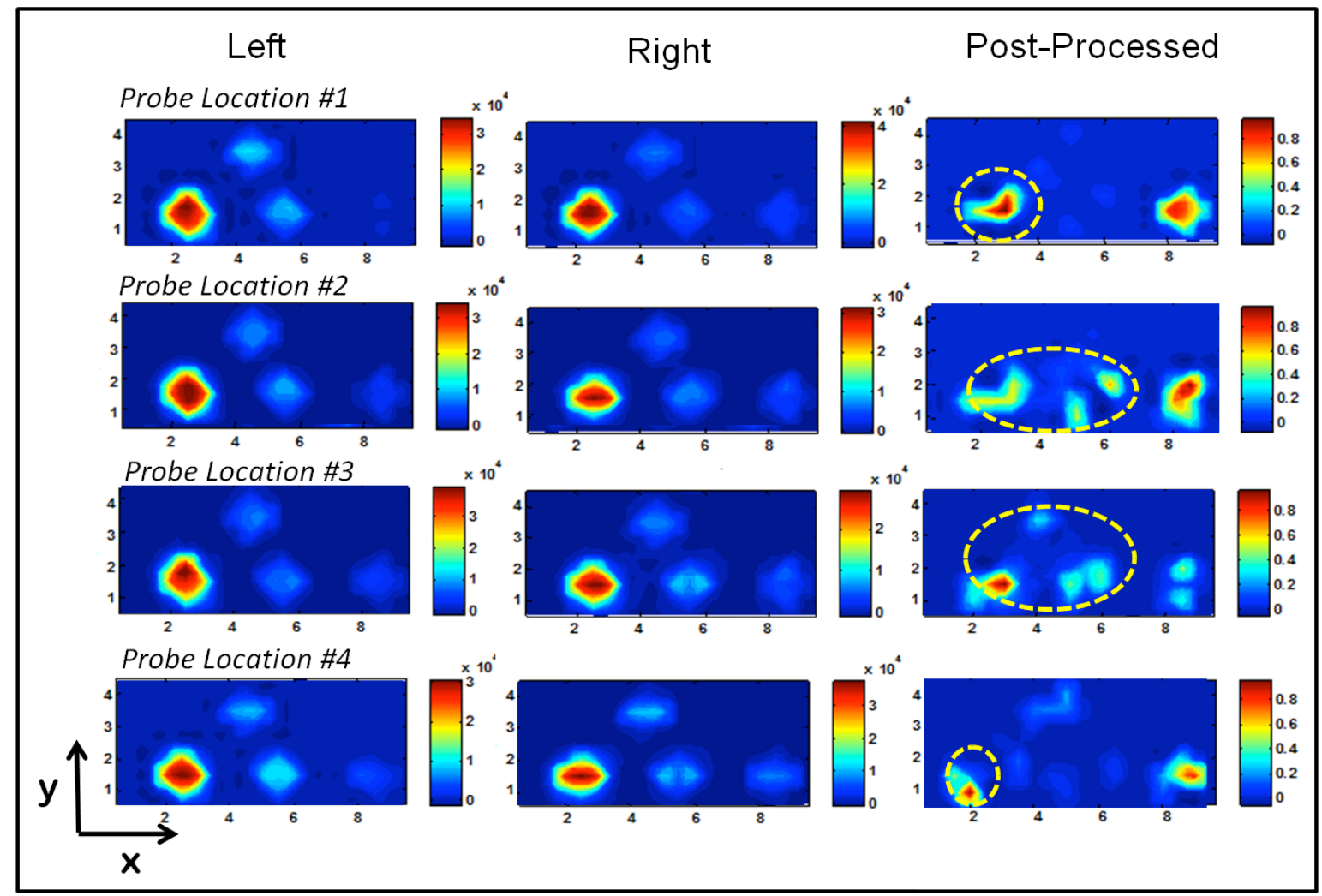

Figure 14.5 Continuous-wave absorption-based optical images collected from subject \#1 at four probe locations (second repetition of images). The images on the left were collected from the left breast tissue and the images in the center were collected from the right breast tissue. The postprocessed images on the right represent the normalized intensity data from the ipsilateral (left) breast subtracted from the normalized intensity data from the contralateral (right) breast showing the optical signal due to absorption as a higher (red) signal in the images. The yellow dotted circle was hand-drawn to represent the region of interest in each image.

Figure 14.6 shows ultrasound images from subject \#1 of suspicious enlarged lymph nodes in the axillary region (indicated in Figure 14.2). Needle biopsy confirmed that at least three lymph nodes in the axillary region contained lymphatic spread of carcinoma. 


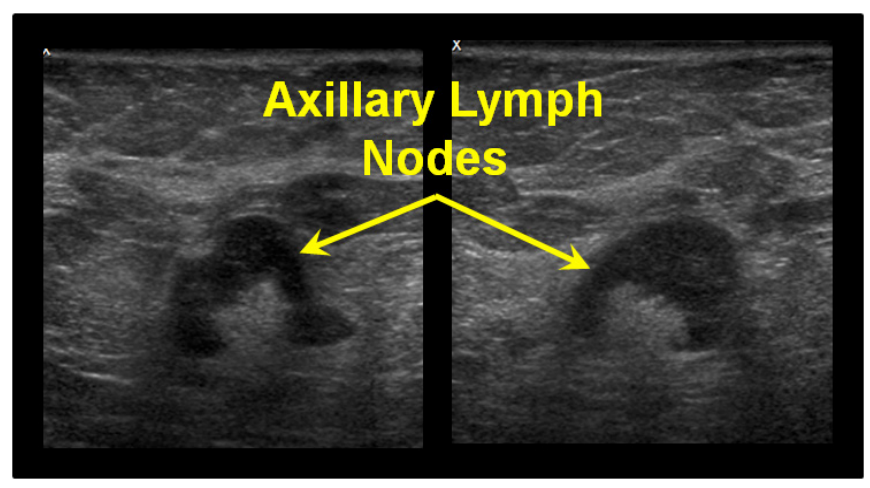

Figure 14.6 Ultrasound images from subject \#1 of suspicious enlarged axillary lymph nodes. (The axillary region is indicated in Figure 14.2.)

The second set of four optical images collected from the outer part of the tissue under the arm in the axillary region and the side of the breast tissue (probe locations indicated in Figure 14.2) are shown in Figure 14.7. The first image from probe location \#1 shows a strong absorption signal (indicated by the dashed yellow circle) which might be from an enlarged axillary lymph node. Additional (weaker) absorption signals in images from probe locations \#2 and \#3 might also indicate regions of affected lymph nodes. The fourth image from probe location \#4 no longer covered the axillary region and was located over the breast tissue. The diffused signal in the fourth image is likely from the abnormal tissue located behind the nipple (similar to the signal in Figure 14.4). Figure 14.8 shows the results from the second repetition of images collected at the same locations and post-processed. The images show similar result to Figure 14.7, however there is also the presence of artifacts because the probe was not in full contact with the tissue. 


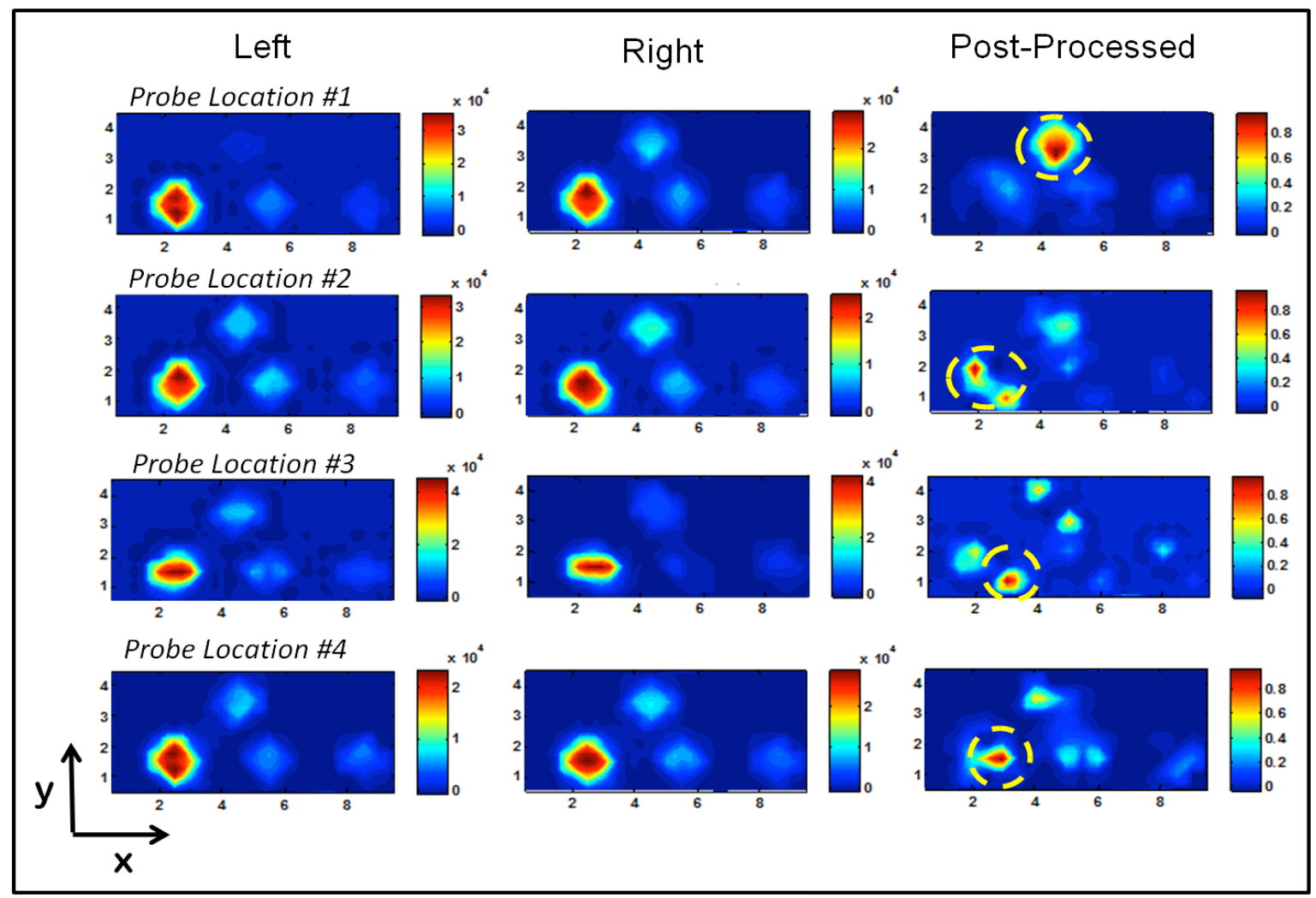

Figure 14.7 Continuous-wave absorption-based optical images collected from subject \#1 at the second set of four probe locations (repetition \#1). The images on the left were collected from the left breast tissue and the images in the center were collected from the right breast tissue. The postprocessed (subtracted) images on the right show the optical signal due to absorption. The yellow dotted circle was hand-drawn to represent the region of interest in each image. 


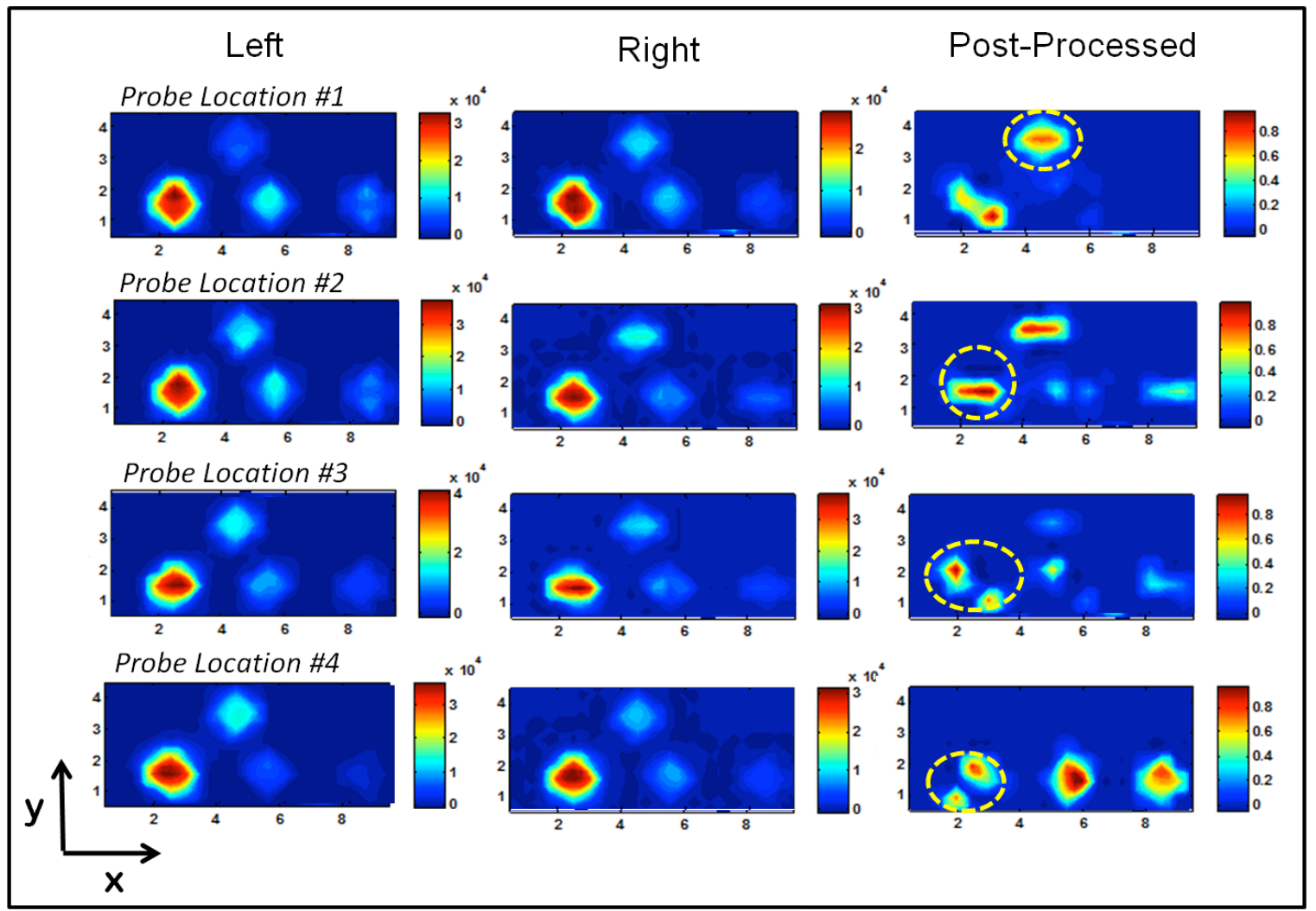

Figure 14.8 Continuous-wave absorption-based optical images collected from subject \#1 at the second set of four probe locations (repetition \#2). The images on the left were collected from the left breast tissue and the images in the center were collected from the right breast tissue. The postprocessed (subtracted) images on the right show the optical signal due to absorption. The yellow dotted circle was hand-drawn to represent the region of interest in each image.

Figure 14.9 shows x-ray mammography images from the left breast of a 51 year-old breast cancer patient (subject \#2). Figure 14.9A is the axial view where the top of the image is toward the left side of the breast and the bottom of the image is toward the right side of the breast. Figure $14.9 \mathrm{~B}$ is the oblique view where the top of the image is toward the patient's head and left side and the bottom of the image is toward the patient's feet and right side. The images show the presence of 3 masses in the outer quadrant of the tissue (indicated by the yellow arrows). The medical reports indicated that two of the masses were cancerous with regions of abnormal tissue between the three masses. 

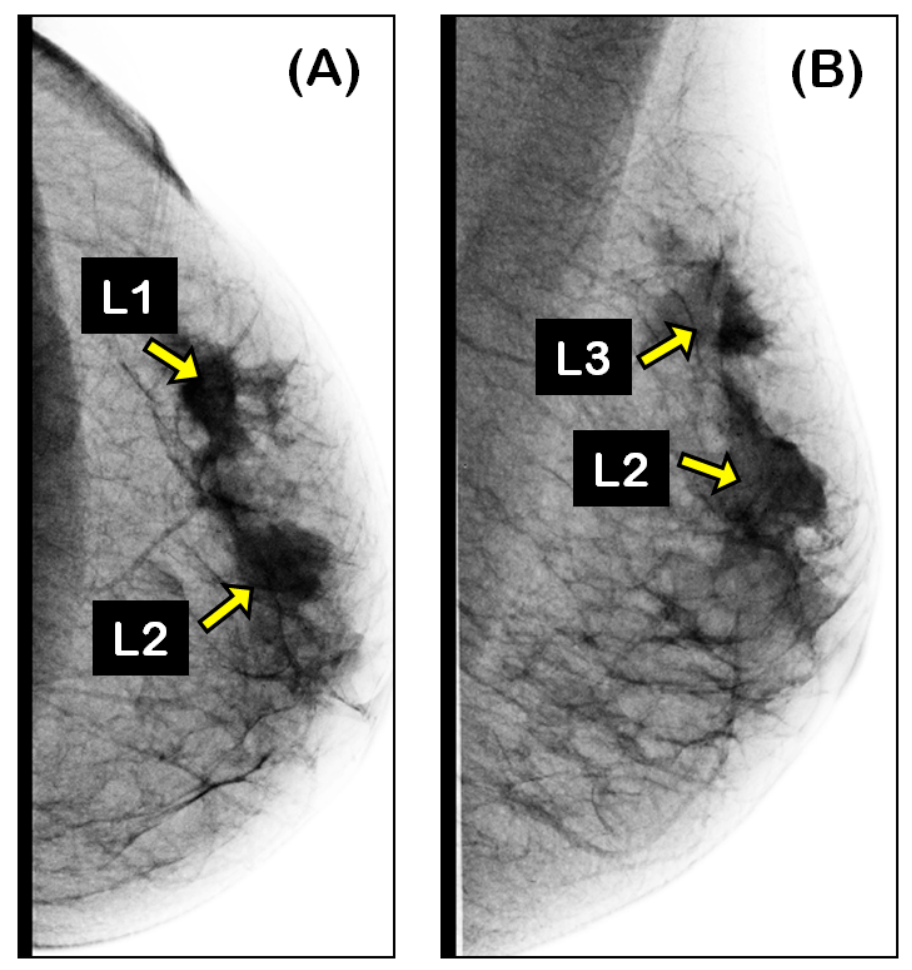

Figure 14.9 X-ray mammography images of the left breast from a 51 year-old breast cancer patient. (A) The top of the image is the left side and the bottom of the image is the right side of the breast tissue. (B) Oblique image where the top of the image is toward the patient's head and left side and the bottom of the image is toward the patient's feet and right side. The yellow arrows indicate 3 lesions (labeled as L1, L2, and L3) located in the 1 o'clock to 2 o'clock region of the breast.

Figure 14.10 shows ultrasound images from the left breast of the same subject. The image in Figure $14.10 \mathrm{~A}$ was collected from the 1 to 2 o'clock position at $2-3 \mathrm{~cm}$ from the nipple and the image in Figure 14.10B was collected from the 1 to 2 o'clock position at $6 \mathrm{~cm}$ from the nipple. The three tumor masses in the outer quadrant of the tissue are indicated by the yellow arrows. 

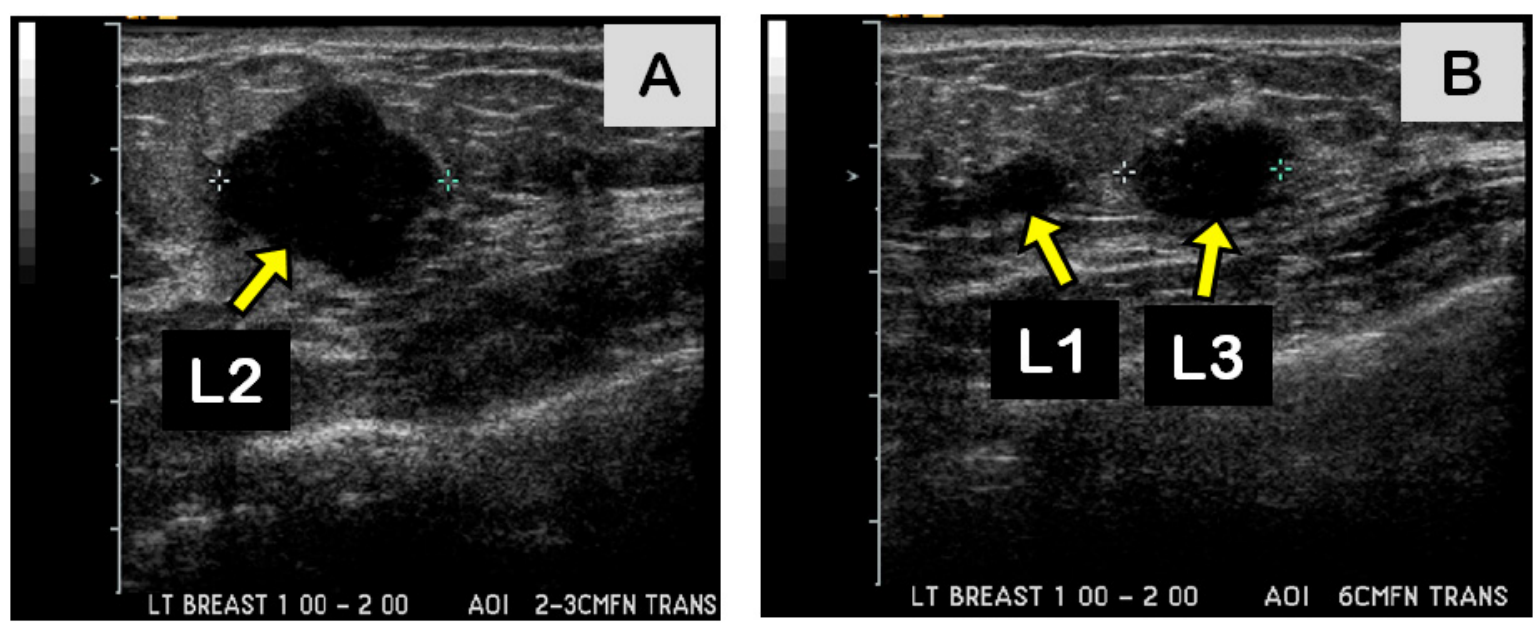

Figure 14.10 Ultrasound images of the left breast from a 51 year-old breast cancer patient. Both images were collected in the 1 to 2 o'clock location where image (A) was 2-3 cm from the nipple and image (B) was $6 \mathrm{~cm}$ from the nipple. The yellow arrows indicate 3 lesions (labeled as L1, L2, ans L3 corresponding to Figure 14.7) located in the 1 o'clock to 2 o'clock region of the breast.

Figure 14.11 shows the continuous-wave absorption-based images collected using the hand-held optical imager. The images on the left were collected from the ipsilateral breast and the images in the center were collected from the contralateral breast. The images on the left were subtracted from the images in the center to yield the resulting postprocessed images on the right. In the post-processed images, the area of higher absorption shows as a higher (or more red) signal. The post-processed images from locations 1-3 show high intensity signal (greater absorption) in the outer quadrant of the left breast tissue which corresponds to the location of the 3 tumor masses indicated in the x-ray mammogram and ultrasound images. The image from location 4 which is from the lowest part of the tissue does not show higher absorption. 


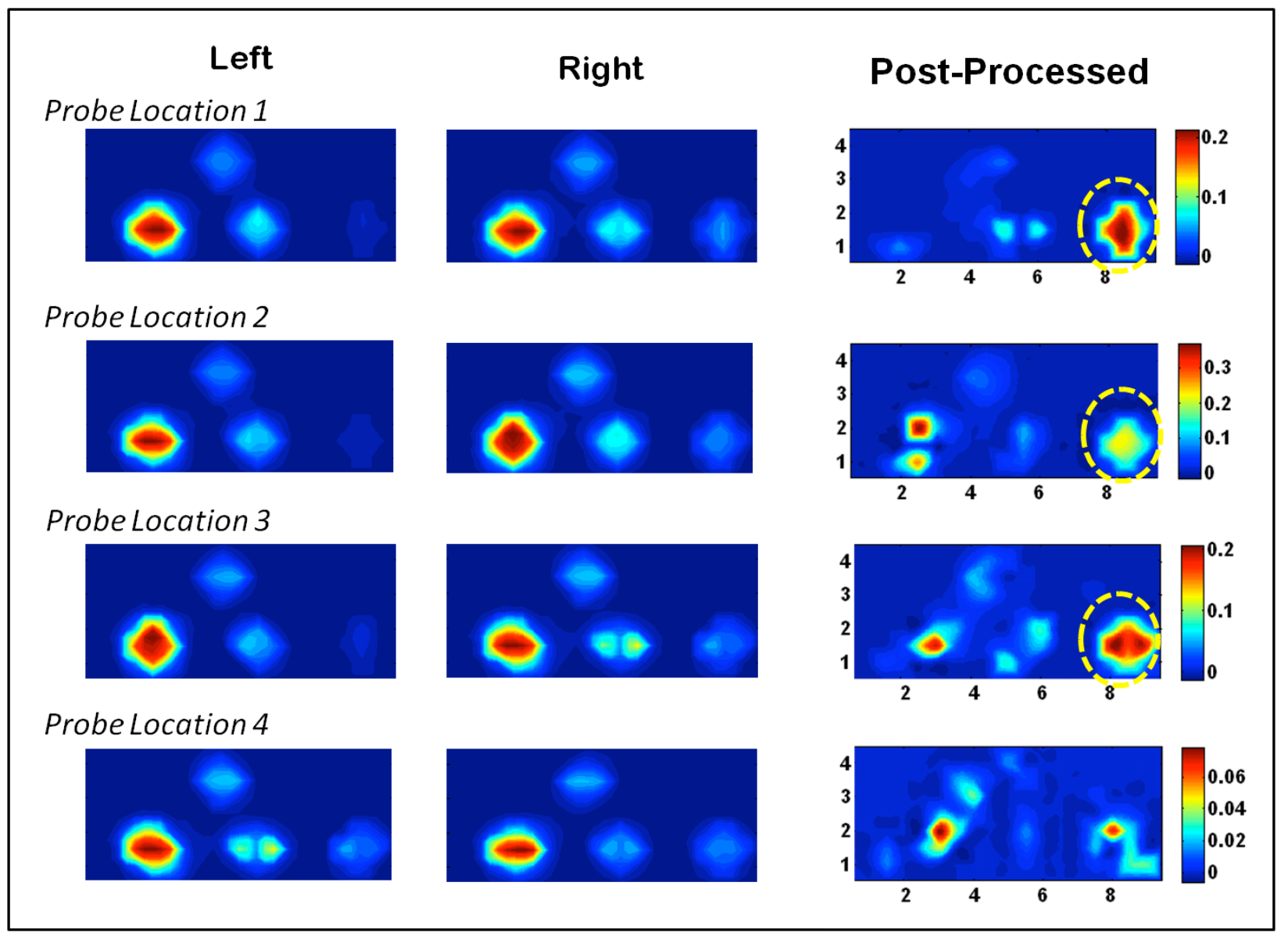

Figure 14.11 Optical images collected from the breast tissue of a 51 year-old breast cancer patient. The images in Set \#1 were collected from the ipsilateral breast and the images in Set \#2 were collected from the contralateral breast. The images from Set \#1 were subtracted from Set \#2 so that the area of higher absorption shows as a higher (or more red) signal in the post-processed images. The yellow dotted circle was hand-drawn to indicate the region of interest.

The subject returned for a second study 42 days following the initial study. The same set of images was collected at the same locations on the breast tissue and the postprocessed images from the two studies are compared in Figure 14.12. The images from study \#2 show intensity signal (absorption) located in the outer quadrant of the left breast tissue; however, the signal is reduced and diffused compared to the images from study \#1. The subject had been undergoing treatment therapy during the time that the images were 
collected and the results in Figure 14.12 might be indicative of reduction of the tumor masses due to the treatment.

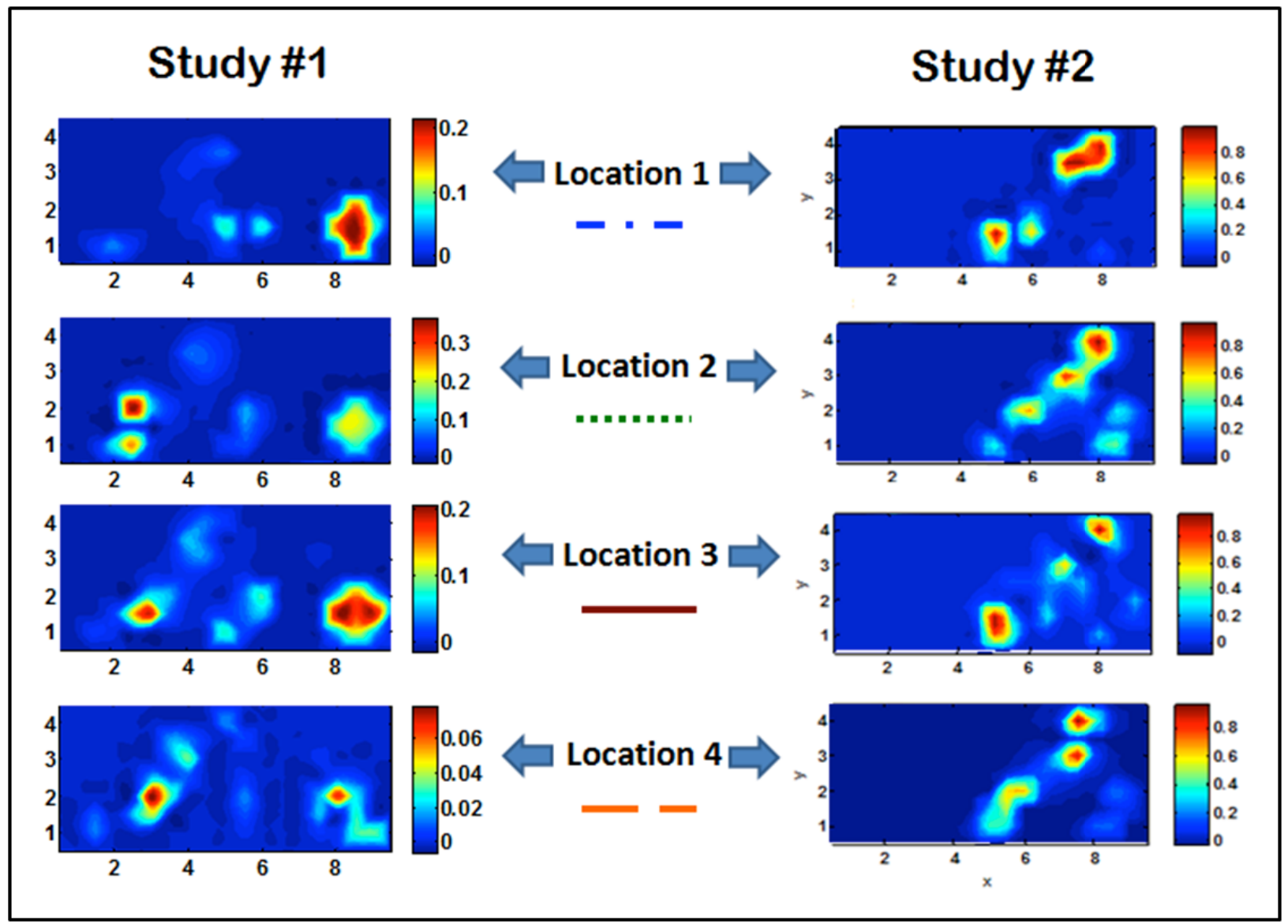

Figure 14.12 Comparison of the results from two different image sets collected on different dates from subject \#2. Study \#2 was performed 42 days after Study \#1 in the same breast cancer subject. Four images were collected at the same probe locations (see Figure 14.1). For each study, images were collected from both the right and left breast tissues and used to subtract the excitation leakage. All images are the final post-processed images. The probe locations were estimated with respect to the nipple location.

\subsection{DISCUSSION}

This chapter presented preliminary data from the first studies performed using our handheld optical imager with actual breast cancer subjects. Subject \#1 had invasive ductal carcinoma with lymphatic spread which was not detected by x-ray mammography. The first 
set of optical images show absorption signal corresponding to the region of abnormal breast tissue indicated in the MRI images. The second set of optical images show absorption signal corresponding to the location of axillary lymph nodes in the region where needle biopsy confirmed lymphatic spread of carcinoma. This study shows the potential of the hand-held device to image cancerous tissues that are undetectable by MRI as well as affected lymph nodes in the axillary region.

The images from subject \#2 show absorption signal corresponding to the location of tumors in a subject undergoing chemotherapy treatment for invasive ductal carcinoma. In a subsequent study with the same subject 42 days following the first study, the signal in the same region was weaker and more diffused. This possibly indicates shrinkage of the tumor masses due to the chemotherapy treatment. These results show the potential of the device in monitoring of response to treatment.

All of the images show artifacts located outside the region of interest. These artifacts might be due to improper contact of the probe with the tissue or the uneven source intensity distribution among the six sources. Additionally, the subtraction post-processing to eliminate excitation leakage was performed using images from two different breast tissues. Hence, differences in the signal distribution due the different composition of the tissue would produce artifacts after subtraction. Filtration methods will be applied in the future to reduce the number of artifacts (Chapter 16).

The hand-held optical imager described herein has potential as a complementary imaging modality for breast cancer imaging in the clinical setting. Currently most breast masses are found by x-ray mammography and/or breast ultrasound and then diagnosed as malignant or benign by surgical biopsy. Since $80 \%$ of biopsies turn out to be negative [2] 
there is a clear need for a method of noninvasively imaging breast tumors to gain more information. Optical imaging provides functional information without any additional harm or discomfort to the patient.

In addition to breast cancer diagnosis, another potential application for optical imaging is for monitoring the progression of tumor response to treatment therapy. Since optical imaging does not use harmful radiation like x-ray and nuclear techniques, the patient can be imaged more frequently. This will reduce the distress of the patients who currently have to wait several months between imaging scans to determine if the treatment therapy is effective. The results shown in the previous section show potential of our hand-held optical imager for use in this application.

\subsection{SUMMARY AND CONCLUSION}

The performance of our hand-held based optical imager was tested in the clinical setting for the first time on actual breast cancer patients. These preliminary results indicate the ability of the device to detect tumors via continuous-wave absorption-based imaging. In addition, multiple studies during treatment therapy demonstrate the potential application of the device to be used to monitor tumor progression at different stages of therapy. Extensive studies will be performed in the future to demonstrate coregistered imaging and $3 \mathrm{D}$ tomography in cancer subjects as well as determine the sensitivity and specificity of the device. 


\section{CHAPTER 15}

\section{Summary and Conclusion: Overall Discussion and Significance of the Work}

A hand-held probe based optical imaging device has been developed in our Optical Imaging Laboratory towards breast cancer imaging. It is distinctive from other hand-held optical devices in its ability to perform 3D tomography in complex human breast tissues via unique self-coregistration capabilities. In the past, our device has demonstrated 3D tomography using manual coregistration in simple cubicle phantoms. The overall goal of this dissertation was towards translation of our device to the clinical setting for 3D tomographic imaging in human breast tissues with complex geometries. A systematic experimental approach was designed and executed to evaluate the performance of the device at each stage of clinical translation. The major objectives of the studies were to demonstrate fast $2 \mathrm{D}$ imaging, coregistered imaging, and 3D tomography in tissue phantoms, in vitro, and in vivo with human subjects.

The first major goal was to perform fast $2 \mathrm{D}$ imaging in order to determine if the device could detect and estimate the 2D location of a target even prior to 3D tomographic analysis. In the clinical setting, fast $2 \mathrm{D}$ imaging is useful to quickly gain a visual picture of the entire breast tissue and determine the specific "areas of interest" to which more in-depth analysis will be applied. For the phantom, in vitro, and in vivo studies performed herein, the term "fast imaging" is used to describe the acquisition and initial post-processing to visualize the target or tumor in a $2 \mathrm{D}$ contour plot. Each image was acquired in near-real time $(\sim 5$ seconds). 
Fast 2D imaging was initially demonstrated in the simple case of homogeneous tissue phantoms composed of $1 \%$ Liposyn solution. A $0.45 \mathrm{~cm}^{3}$ spherical target filled with $1 \mu \mathrm{M}$ Indocyanine green (ICG) fluorescent contrast agent was detectable up to a depth of $2.5 \mathrm{~cm}$ under perfect uptake conditions (Section 8.3.1). The next step was to introduce a heterogeneous background which better mimicked the properties of actual human tissue. Studies were performed in vitro using minced chicken breast combined with 1\% Liposyn. Spherical targets of 0.45 and $0.23 \mathrm{~cm}^{3}$ volume filled with $1 \mu \mathrm{M}$ ICG were detectable up to $2.0 \mathrm{~cm}$ deep under perfect uptake conditions (Section 8.3.2). The final step was to see if a target could be detected through actual human breast tissue. Healthy female subjects above age 21 were recruited for the IRB-approved study. Spherical targets of 0.45 and $0.23 \mathrm{~cm}^{3}$ filled with $1 \mu \mathrm{M}$ ICG were noninvasively placed underneath the flap of the breast tissue and were detected through $\sim 2.5 \mathrm{~cm}$ of human breast tissue (Section 11.3).

The fast $2 \mathrm{D}$ imaging studies demonstrated that small targets $\left(0.23-0.45 \mathrm{~cm}^{3}\right)$ could be detected in near-real time using our hand-held optical imager in tissue phantoms, in vitro, and in vivo. It is interesting to note that the deepest target detected for in vitro studies was at a depth of $2.0 \mathrm{~cm}$, whereas in the more complex human subject case, the target was detected through $\sim 2.5 \mathrm{~cm}$ of tissue. Chicken breast tissue is composed primarily of muscle whereas human breast tissue has various components such as mammary glands, milk ducts, and fatty tissue. Hence, while phantom and in vitro experiments are useful for preliminary investigations of the capabilities of a device and optimization, they do not accurately predict what result will occur in a true human subject case. The scattering coefficient of the in vitro phantom ranged between 6 and $12 \mathrm{~cm}^{-1}$ which is close to the average value of human breast tissue $\left(\sim 8 \mathrm{~cm}^{-1}\right) \cdot$ [134] Extensive clinical studies are required to fully define the performance 
of the device in order to account for the wide variability between subjects. The research performed for this dissertation has effectively translated the device from the laboratory testing stage to the clinical studies phase and the imaging set-up and protocol have been methodically structured to be conducive now for a continuous flow of human subject studies.

Fast 2D imaging in near-real time is useful to gain an initial estimation of the area of interest to be interrogated in more detail. In order to proceed with further analysis, it is necessary to determine the location of the probe image with reference to the geometry of the tissue (i.e. coregister the images). The second major goal was to demonstrate automated coregistered imaging which is necessary to perform 3D tomography. The optical tomography studies performed by other research groups typically used bulky instrumentation with the breast placed within a fixed geometry (between two compression plates or in a ring).[22,110] The fixed location and geometry enables the image location relative to the breast geometry to be known, allowing for tomography. Only one research group has demonstrated 3D tomography using a dual optical/ultrasound hand-held device.[66] The additional data from the ultrasound was used as structural a priori information for tomography. Our device is unique in that is has self-coregistration capabilities enabling 3D tomography to be performed using a hand-held optical-only device, without requiring a second imaging modality.

An acoustic based tracking system was implemented with the hand-held device and automated coregistration software (developed in-house using MATLAB/LabVIEW) was used to perform self-coregistered imaging. Automated coregistered imaging was quantitatively validated in tissue phantoms and the average distance off between the 
measured location and true location of the probe with respect to the phantom was $\sim 0.19$ $\mathrm{cm}$. The discrepancy was attributed to instability of the tracking system. Coregistered imaging was performed in tissue phantoms and in vitro where multiple coregistered images were summed in order to increase the signal from the target while relatively reducing the random artifacts enabling deeper target detection. The depth of detection for tissue phantoms was improved from 2.5 to $3.5 \mathrm{~cm}$ in tissue phantoms under perfect uptake and 3.0 cm under imperfect ( $\mathrm{T}: \mathrm{B}=100: 1)$ uptake. The depth of detection in vitro was improved from $2.0 \mathrm{~cm}$ to $3.5 \mathrm{~cm}$ under perfect uptake and $2.5 \mathrm{~cm}$ under imperfect ( $\mathrm{T}: \mathrm{B}=100: 1)$ uptake for target volumes of $0.23-0.45 \mathrm{~cm}^{3}$ (Section 9.3).

Coregistered imaging was performed in vivo in healthy human subjects. Tracking of the probe was quantitatively validated by automatically inserting reference points into the 3D meshed geometry via code developed in MATLAB. The average total distance off between the measured location and true location was $\sim 1 \mathrm{~cm}$. The much larger discrepancy (compared to the discrepancy in phantoms) was attributed to movement of the subject, deformation of the breast tissue, and probe-operator error. The imaging set-up was modified to minimize human error during in vivo imaging (by scanning and imaging the subject in supine position at a $45^{\circ}$ degree angle, Section 12.2.3). Currently efforts are made by others in our lab to develop an optical tracking system to reduce the error due instability. Future work will involve testing the total reduction in error using the new tracking method (as described in Chapter 16). Automated coregistered imaging was performed on a healthy human subject with a spherical target $\left(0.45 \mathrm{~cm}^{3}\right)$ filled with $1 \mu \mathrm{M}$ ICG placed noninvasively underneath the flap of the breast tissue. Due to increased instability of the acoustic tracking system, a manual coregistration approach was temporarily implemented and performed in a 
healthy human subject with a $0.45 \mathrm{~cm}^{3}$ spherical target filled with $0.08 \%$ and $0.04 \%$ India ink placed underneath the flap of the breast tissue. The results demonstrated the ability to detect both fluorescent and absorption based targets through human breast tissue and coregister the image at the appropriate tissue location using both automated and manual coregistered imaging (Section 12.3).

Coregistered images provide the appropriate positional information to enable 3D tomographic analysis. The third major goal was to perform 3D tomography in tissue phantoms, in vitro, and in vivo using our hand-held optical imager. Initially, 3D tomography was performed using manual coregistration of the fast $2 \mathrm{D}$ images of fluorescent targets in vitro with the simple cubicle geometries. The targets were recovered close to the true location; however, the recovered optical property $\left(\mu_{\text {axf }}\right)$ values were very low which could be due to the highly diffused signal from depths of $2-2.5 \mathrm{~cm}$ in a heterogeneous background (Section 10.3.1). Subsequent tomography studies were performed using the summated coregistered data. The reconstruction results showed many artifacts and it could not be conclusively determined that the target was recovered (Section 10.3.2). Future work will be performed to apply some filtration techniques and to use frequency-domain data for the reconstructions which provides additional phase information that is not present in CW imaging employed in the current research studies (as described in Chapter 16).

The major challenge in performing 3D tomography in human tissue as opposed to simple phantoms is to generate the discretized volume geometry (or mesh) for the human breast tissue. For simple cubicle phantoms, the geometry and mesh were generated within a single software (Gambit) and the same mesh was used for each phantom reconstruction. In the case of human subject studies, the various breast tissue geometries must be acquired for 
each subject and discretized into tetrahedral volume mesh. Complications occurred in the process of trying to import an acquired surface scan into appropriate software to generate the $3 \mathrm{D}$ volume and mesh. Meshing softwares are typically designed such that a geometry is both generated and meshed within that same software. However, our application presented a unique complication in that we required an externally acquired complex geometry to be imported into the software and used to generate a 3D volume and mesh. A ten-step procedure was developed to acquire the tetrahedral volume mesh of the breast tissue and extract the experimental information corresponding to the individual volume breast mesh needed to input into the reconstruction algorithm (Section 13.2.1).

Three-dimensional (3D) tomography was performed using the data from a healthy human subject with a fluorescent target placed underneath the flap of the breast tissue. The signal from the target was recovered close to the true location along with artifacts (Section 13.3). The artifacts could be caused by improper contact of the probe with the tissue surface, uneven intensity distribution among the six sources in the probe face, or noise in the experimental data. Efforts will be made to reduce the artifacts as part of the future work (Chapter 16). The result demonstrates for the first time the feasibility of performing 3D tomography in breast tissue of a human subject using a hand-held optical imager.

The final stage of the dissertation involved preliminary studies using the hand-held optical imager on actual breast cancer subjects. Diffuse optical imaging studies were performed in two female patients with invasive ductal carcinoma. The images showed higher absorption signals in the regions of the tumor locations along with artifacts (Section 14.3). The results demonstrate the potential of the device to detect the presence of cancer within a known region of interest which has clinical application towards prognostic imaging 
since the location of the tumor versus surrounding artifacts will be known. In order for the device to be used for diagnostic purposes, the artifacts that have occurred in the images must be minimized. Future work will involve efforts to improve the instrumentation as well as the imaging procedure in order to reduce the artifacts.

There are several unique advantages of our hand-held optical imager. The probe contains six illumination points and 165 detection points (distributed over a 4x9 cm) area which illuminate and collect the signal simultaneously to enable rapid data acquisition in large volumes of tissue. The probe is designed with a flexible face to contour to the curvature of the breast tissue allowing maximum patient comfort. Self-coregistration facilities are implemented with the probe to accurately position the image on the complex 3D breast tissue geometry. The hand-held optical imager is the only one of its kind that is capable of performing 3D tomography in human breast tissue.

There are also several limitations of the hand-held device. The probe uses a subsurface based imaging configuration which limits the collected signal to reflectance measurements only. The physics of reflectance based imaging leads to recovery of targets closer to the imaging surface limiting the ability to accurately recover the true target depth. The acoustic tracking system proved unstable causing error in image coregistration. The bulky design of the bench-top hand-held imaging device is not portable and operatorfriendly for efficient bedside imaging. The next chapter will describe the efforts to overcome these limitations as part of our ongoing and future work.

The significance of the work presented in this dissertation is towards the clinical translation of a hand-held optical imager for bedside imaging and 3D tomography of human breast tissue. The process of clinical translation of our hand-held optical imager involved 
modification of the experimental set-up and method from bench-top phantom studies to bedside human subject imaging. The performance of the device was evaluated using progressively more complex subjects, beginning with the simple case of homogeneous tissue phantoms, then using more complex heterogeneous in vitro phantoms, and finally testing in actual human breast tissue. While phantom and in vitro studies are useful for gaining some insight into how the device will perform, extensive human subject studies are integral to gaining a true assessment of the device performance in the clinical setting. The hand-held optical imager has potential impact for women with breast cancer by providing a safe and patient comfortable method for functional imaging of breast tissue towards cancer diagnosis and prognosis. 


\section{CHAPTER 16 \\ Ongoing and Future Work}

The work performed for this dissertation has brought the development of our handheld optical imager from the stage of bench-top testing in simple cubical phantoms to the stage of performing extensive bedside human subject studies. Through collaboration with a breast surgeon and a cancer center, we are continuously recruiting breast cancer patients to participate in our research studies. Parallely, we will continue to perform studies in healthy subjects as a control group, and in the future we will recruit subjects with various types of non-cancerous breast lesions (e.g. cysts, fibroadenoma, etc.) Our imager must be tested on many subjects over a wide range of demographics in order to determine the sensitivity, specificity, positive predictive value, and negative predictive value of the device.

The three major aspects that the ongoing and future work will focus on to improve the imaging results are (i) instrumentation, (ii) data acquisition, and (iii) post-processing. The current limitations in the instrumentation are the bulky bench-top design of the optical imaging system, the heavy and awkward hand-held probe, and the unstable tracking system. In order to address the limitations in the bulky bench-top system and probe design, a second generation of the hand-held optical imager is being developed which has a more sleek and portable design. The instrumentation is more compact and fits on a portable cart for ease of bedside imaging. The hand-held-probe has a two-piece design which is smaller and lighter for comfort and better control of operation as well as the addition of transillumination based measurements to improve target depth recovery. Another limitation of the first generation device is the uneven source intensity distribution which causes artifacts in the images. The 
second generation device will contain six independent laser diodes to enable a uniform source intensity distribution. This second generation device will be tested extensively in healthy human subjects and breast cancer patients.

Another limitation in the instrumentation is the instability of the tracking system,. An alternate optical-based tracking system is being developed to enable more accurate image coregistration. Once the optical-based tracking system is developed, coregistered imaging in human subjects will be reevaluated quantitatively to determine how much the discrepancy between true and measured probe locations was reduced by the combined efforts of (i) improving the stability of the tracking system and (ii) modifying the imaging set-up to reduce human error.

Efforts will be made to improve the methods of data acquisition in order to systemize the imaging approach across multiple human subjects. To this end, a structured imaging approach will be implemented which uses a template of predefined locations of the probe which are marked on each breast tissue to ensure continuity of imaging among repetitions with each subject and across multiple subjects. In order to reduce the movement and conformation of the breast tissue, an optically clear custom-built garment will be used with the Generation II system to confine the breast tissue. The hand-held 3D scanner will be used as the motorized system with the subject seated in an upright position.

Post-processing techniques will also be applied as part of the future work in order to minimize the presence of artifacts. Filtration will be applied to the data to try to remove noisy data points that contribute to artifacts. Since each image represents an average of 510 repetitions, there may be noisy data in one of the repetitions which can be identified by the magnitude of the standard deviation and removed.[157] 
Several challenges were encountered and lessons were learned throughout the dissertation process. One lesson is the importance of maintaining a systematic experimental approach at every stage in the research. If a new method or idea is applied that produces an improved result it can be tempting to immediately try it to the most extreme case to see the improvement. However, any new method must be systematically applied at each incremental stage in order to see the effect on the experimental results. During preparation for human subject studies, experience was gained while assisting in writing the IRB proposal. It can be a strenuous process and it is important to think ahead and cover every possible aspect of the experimental procedure. The procedure will naturally be altered as limitations are discovered and more effective methods are implemented and every detail must be documented in the IRB protocol. There is also the challenge of collaborating with other institutions requiring an additional IRB approval from the other institutions which can be a lengthy process.

Several major lessons were learned during this process occurred during the transition from phantom and in vitro studies to human subject studies. During the experience acquired throughout the dissertation research, many things have been discovered that must be taken into consideration when performing human subject studies as opposed to phantom studies. In phantom studies there is much more control over the experiment in that the phantom is made the same way each time, and once it is set in place it remains fixed for the extent that is required during data collection and troubleshooting. With a human subject, there is much more variability and many more unknowns. The tissues of human subjects differ widely in size and density. The imaging procedure must be generalized enough to account for the differences. Although the subject is asked to remain still during the imaging procedure, there 
inevitably will be some movement which can impact the results. Human subject studies must be performed in a limited amount of time, so if problems occur, troubleshooting must be done quickly on the spot or the data cannot be collected at that time. Another lesson is that the behavioral conduct of the researchers performing the experiment must be much more formal than during phantom studies. Problems inevitably occur and it is important not to cause tension or panic in subject who does not understand the operation of the instrumentation and might worry that the problem would affect them. It is also important to maintain awareness of the demographics in the area where the subjects will be recruited. The studies described here were performed in an area that has a high Hispanic population and some of the subjects spoke very limited English. It was necessary to have the consent forms prepared in both English and Spanish so the subject could be informed in their preferred language. Another aspect that is required for human subject studies is the confidentiality of sensitive information. The identity of the subject is never associated with the data collected, and the medical records must be kept locked to protect the privacy of the subject.

The lessons learned during the dissertation process will be incorporated in the ongoing and future work with the hand-held optical imager. Clinical studies with patient trials are integral to the translation of any device. Extensive clinical trials will provide a broader scope of the device capabilities and expected performance in a true clinical setting. 


\section{REFERENCES}

1. American Cancer Society, Breast Cancer Facts and Figures, 2009.

2. American Cancer Society http://www.cancer.org/

3. Ge J, Zhu B, Regalado S, Godavarty A, "Three-dimensional fluorescence-enhanced optical tomography using a hand-held probe based imaging system.” Med. Phy. 2008, 35(7):3354-3363.

4. X. Intes, B. C. (2005). "Non-PET functional imaging techniques: optical." Radiol Clin N Am 43: 221-234.

5. A. Yodh, B. C. (1995). "Spectroscopy and Imaging with Diffusing Light." Physics Today 48(3): 34-40.

6. $\quad$ R. Weissleder (2001). "A clearer vision for in vivo imaging." Nature Biotechnology 19: 316-317.

7. Fantini S, Walker SA, Franceschini MA, Kaschke M, Schlag PM, Moesta KT, "Assessment of the size, position, and optical properties of breast tumors in vivo by noninvasive optical methods," Appl Opt. 1998, 37: 1982-1989.

8. Eppstein MJ, Hawrysz DJ, Godavarty A, Sevick-Muraca EM, "Three-dimensional near-infrared fluorescence tomography with Bayesian methodologies for image reconstruction from sparse and noisy data sets," Proc. Natl. Acad. Sci. USA, 2002, 99: 9619-9624.

9. Milstein B, Stott JJ, Oh S, Boas DA, Millane RP, Bouman CA, Webb KJ, "Fluorescence optical diffusion tomography using multiple-frequency data," J. Opt. Soc. Am. A., 2004, 21 ( 6) : 1035-1049.

10. Lee J, Sevick-Muraca EM, “3-D Fluorescence enhanced optical tomography using references frequency-domain photon migration measurements at emission and excitation measurements," J. Opt. Soc. Am. A., 2002, 19: 759-771.

11. Quaresima V, Matcher SJ, Ferrari M, "Identification and quantification of intrinsic optical contrast for near-infrared mammography," Photochem. Photobiol., 1998, 67: 414.

12. Li X, Culver J, Durduran T, Chance B, AG Yodh, Pattanayak DN, "Diffraction tomography with diffuse-photon density waves: clinical studies and background subtraction," in Biomedical Optical Spectroscopy and Diagnostics / Therapeutic Laser Applications, E. Sevick-Muraca, J. Izatt, and M. Ediger, eds., Vol. 22 of OSA Trends in Optics and Photonics Series (Optical Society of America, 1998), paper JTuA3. 
13. Ntziachristos V, Weissleder R, "Experimental three-dimensional fluorescence reconstruction of diffuse media by use of a normalized Born approximation," Opt. Lett., 2001, 26: 893-895.

14. Yang $\mathrm{Y}$, Iftimia $\mathrm{N}, \mathrm{Xu} \mathrm{Y}$, Jiang $\mathrm{H}$, "Frequency-domain fluorescent diffusion tomography of turbid media and in vivo tissues," in Optical Tomography and Spectroscopy of Tissue IV, B. Chance, R. R. Alfano, B. J. Tromberg, eds., Proc. Soc. Photo-Opt. Instrum. Eng., 2001, 4250: 537-545.

15. Ntziachristos V, Bremer C, Weissleder R, "Fluorescence imaging with near-infrared light: new technological advances that enable in vivo molecular imaging," Eur Radiol, 2003, 13:195-208.

16. Ntziachristos V, Weissleder R, "Charge-coupled-device based scanner for tomography of fluorescent near-infrared probes in turbid media," Med. Phys., 2002, 29: 803-809.

17. Ntziachristos V, Bremer C, Tung C, Weissleder R, "Imaging cathepsin B up-regulation in HT-1080 tumor models using fluorescence-mediated molecular tomography (FMT)," Acad. Radiol, 2002, 9(suppl 2):S323-S325.

18. Schulz RB, Ripoll J, Ntziachristos V, "Noncontact optical tomography of turbid media , "Opt. Lett., 2003, 28(18): 1701-1703.

19. Ntziachristos V, Schellenberger EA, Ripoll J, Yessayan D, Graves E, Bogdanov A, Josephson JL, Weissleder R, "Visualization of antitumor treatment by means of fluorescence molecular tomography with an annexin V-Cy5.5 conjugate," Proc. Natl. Acad. Sci. USA, 2004, 101 (33): 12294-12299.

20. Schulz RB, Ripoll J, Ntziachristos V, "Experimental fluorescence tomography of tissues with noncontact measurements," IEEE Trans. Med. Imaging, 2004, 23( 4): 492-500.

21. Bremer C, Ntziachristos V, Weitkamp B, Theilmeier G, Heindel W, Weissleder R, "Optical imaging of spontaneous breast tumors using protease sensing 'smart' optical probes,” Invest Radiol, 2005, 40(6):321-7.

22. Colak SB, Papaioannou DG, t'Hooft GW, van der Mark MB, Schomberg H, Paasschens JCJ, Melissen JB, van Asten NJ, "Tomographic image reconstruction from optical projections in light-diffusing media,” Appl. Opt., 1997, 36: 180-213.

23. Godavarty A, Thompson AB, Roy R, Eppstein MJ, Zhang C, Gurfinkel M, SevickMuraca EM, "Diagnostic imaging of breast cancer using fluorescence-enhanced optical tomography: phantom studies," J Biomed Opt: Special edition on Biomedical Optics and Women's Health, 2004, 9(3): 488-496. 
24. Godavarty A, Eppstein MJ, Zhang C, Theru S, Thompson AB, Gurfinkel M, SevickMuraca EM, "Fluorescence-enhanced optical imaging in large tissue volumes using a gain modulated ICCD camera," Phys. Med. Biol., 2003, 48: 1701-1720.

25. Godavarty A, Zhang C, Eppstein MJ, Sevick-Muraca EM, "Fluorescence-enhanced optical imaging of large phantoms using single and simultaneous dual point illumination geometries," Med. Phys., 2004, 31: 183-90.

26. Godavarty A, Sevick-Muraca EM, Eppstein MJ, "Three-dimensional fluorescence lifetime tomography," Med. Phys., 2005, 32: 992-1000.

27. Jayachandran B, Ge J, Regalado S, Godavarty A, "Design and development of a handheld optical probe toward fluorescence diagnostic imaging." J Biomed Opt, 2007, 12(5): 054014.

28. Zhu B, Eppstein MJ, Sevick-Muraca EM, Godavarty A, "Noise pre-filtering techniques in fluorescence-enhanced optical tomography." Opt. Express, 2007, 15(18): 1128511300 .

29. S.J. Erickson and A. Godavarty. "Hand-Held Based Near-Infrared Optical Imaging Systems: A Review" Medical Engineering and Physics, 2009, 31, 495-509.

30. Zhu Q, Chen NG, Piao DQ, Guo PY, Ding XH, "Design of near-infrared imaging probe with the assistance of ultrasound localization," Appl. Opt., 2001, 40(19), 32883303.

31. Tromberg BJ, "Optical scanning and breast cancer," Acad. Radiol, 2005, 12(8), 923924.

32. Pham TH, Coquoz O, Fishkin JB, Anderson E, Tromberg BJ, "Broad bandwidth frequency domain instrument for quantitative tissue optical spectroscopy," Rev. Sci. Instrum., 2000, 71(6), 2500-2513.

33. Tromberg B, Coquoz O, Fishkin JB, Pham T, Anderson ER, Butler J, Cahn M, Gross JD, Venugopalan V, Pham D, "Non-invasive measurements of breast tissue optical properties using frequency-domain photon migration." Philos Trans R Soc Lond B Biol Sci, 1997, 352: 661-668.

34. Lanning R, Tromberg, "Non-invasive characterization of breast cancer using near infrared optical spectroscopy." The UCI Undergraduate Research Journal II, 1999, 4349.

35. Tromberg BJ, Shah N., Lanning R, Cerussi A, Espinoza J, Pham T, Svaasand L, Butler $\mathrm{J}$, "Noninvasive in vivo characterization of breast tumors using photon migration spectroscopy." Neoplasia, 2000, 2(1-2): 26-40. 
36. Holboke MJ, Tromberg BJ, Li X, Shah N, Fishkin J, Kidney D, Butler J, Chance B, Yodh AG, "Three-dimensional diffuse optical mammography with ultrasound localization in a human subject." J Biomed Opt, 2000, 5(2): 237-247.

37. Cerussi AE, Berger AJ, Bevilacqua F, Shah N, Jakubowski D, Butler J, Holcombe RF, Tromberg BJ, "Sources of absorption and scattering contrast for near-infrared optical mammography." Acad Radiol, 2001, 8: 211-218.

38. Shah N, Cerussi A, Eker C, Espinoza J, Butler J, Fishkin J, Hornung R, Tromberg B, "Noninvasive functional optical spectroscopy of human breast tissue," Proc. Natl. Acad. Sci. USA, 2001, 98(8), 4420-4425.

39. Shah N, Cerussi AE, Jakubowski D, Hsiang D, Butler J, Tromberg BJ, "Spatial variations in optical and physiological properties of healthy breast tissue." J Biomed Opt, 2004, 9(3): 534-540.

40. Bevilacqua F, Berger AJ., Cerussi AE, Jakubowski D, Tromberg BJ, "Broadband absorption spectroscopy in turbid media by combined frequency-domain and steadystate methods." Appl. Opt., 2000, 39(34): 6498-6507.

41. Cerussi AE, Jakubowski D, Shah N, Bevilacqua F, Lanning R, Berger AJ, Hsiang D, Butler J, Holcombe RF, Tromberg BJ, "Spectroscopy enhances the information content of optical mammography." J Biomed Opt, 2002, 7(1): 60-71.

42. Jakubowski DB, Cerussi AE, Bevilacqua F, Shah N, Hsiang D, Butler J, Tromberg BJ, "Monitoring neoadjuvant chemotherapy in breast cancer using quantitative diffuse optical spectroscopy: a case study," J Biomed Opt, 2004, 9(1), 230-238.

43. Shah N, Gibbs J, Wolverton D, Cerussi A, Hylton N, Tromberg BJ, "Combined diffuse optical spectroscopy and contrast-enhanced magnetic resonance imaging for monitoring breast cancer neoadjuvant chemotherapy: a case study." J Biomed Opt, 2005, 10(5): 051503.

44. Hsiang D, Shah N, Yu H, Su MY, Cerussi A, Butler J, Baick C, Mehta R, Nacioglu O, Tromberg B, "Coregistration of dynamic contrast enhanced MRI and broadband diffuse optical spectroscopy for characterizing breast cancer." Technol Cancer Res Treat, 2005, 4(5): 549-558.

45. Cerussi A, Shah N, Hsiang D, Durkin A, Butler J, Tromberg BJ, "In vivo absorption, scattering, and physiologic properties of 58 malignant breast tumors determined by broadband diffuse optical spectroscopy." J Biomed Opt, 2006, 11(4): 044005.

46. Cerussi A, Hsiang D, Shah N, Mehta R, Durkin A, Butler J, Tromberg BJ, "Predicting response to breast cancer neoadjuvant chemotherapy using diffuse optical spectroscopy." Proc. Natl. Acad. Sci. USA, 2007, 104(10): 4014-4019. 
47. No KS, Chou PH, "Mini-FDPM and heterodyne mini-FDPM: handheld non-invasive breast cancer detectors based on frequency-domain photon migration." IEEE Transactions on Circuits and Systems-I: Regular Papers, December 2005, 52(12): 2672-2685.

48. No KS, Xie Q, Kwong R, Cerussi A, Tromberg BJ Chou P, "HBS: a handheld breast cancer detector based on frequency domain photon migration with full heterodyne." Proc. IEEE BioCAS, Nov 29 - Dec 1, 2006. The British Library, London..

49. No KS, Xie Q, Chou PH, Kwong R, Cerussi A, Tromberg BJ, "In vivo breast cancer measurement with a handheld laser breast scanner." in the 50th IEEE International Midwest Symposium on Circuits and Systems (MWSCAS), August 5-8, 2007. Montreal Marriott Chateau Champlain Hotel.

50. Chance B, Nioka S, Zhang J, Conant EF, Hwang E, Briest S, Orel SG, Schnall MD, Czerniecki BJ, "Breast cancer detection based on incremental biochemical and physiological properties of breast cancers: A six-year, two-site study," Acad. Radiol, 2005, 12(8), 925-933.

51. Nioka S, Chance B, "NIR spectroscopic detection of breast cancer." Technol Cancer Res Treat, 2005, 4(5): 497-512.

52. Sao V, Pourrezaei K, Akin A, Ayaz H, "Breast tumor imaging using NIR LED based handheld continuous-wave imager." Proc. of the IEEE 29th Annual NE Bioeng. Conf., Reisman S., Foulds R., Mantilla B., eds. March 22-23, 2003 55-56.

53. Chance B, Zhao Z, Wen S, Chen Y, "Simple ac circuit for breast cancer detection and object detection.” Rev. Sci. Instr., 2006, 77: 064301.

54. Liu Q, Luo Q, Chance B, "2D phased array fluorescence wireless localizer in breast cancer detection." IEEE/EMBS International Summer School on Medical Devices and Biosensors (ISSS-MD), June 26 - July 2, 2004, 71-73.

55. Xu R, Qiang B, Mao J, "Near infrared imaging of tissue heterogeneity: probe design and sensitivity analysis." IEEE Eng. in Med. and Biol. 27th Annual Conference, Shanghai, China September 1-4, 2005.

56. Xu RX, Olsen JO, Povoski SP, Yee LD, Mao J, "Localization and functional parameter reconstruction of suspicious breast lesions by near infrared/ultrasound dual mode imaging." IEEE Eng. in Med. and Biol. 27th Annual Conference, Shanghai, China September 1-4, 2005.

57. Xu JRX, Qiang B, Mao JJ, Povoski SP, "Development of a handheld near infrared imager for dynamic characterization of in vivo biological tissue systems." Appl. Opt., 2007, 46: 7442-7451. 
58. Choe R "Diffuse optical tomography and spectroscopy of breast cancer and fetal brain." PhD thesis, University of Pennsylvania, 2005.

59. Durduran T, Choe R, Yu G, Zhou C, Tchou JC, Czerniecki BJ, Yodh AG, "Diffuse optical measurement of blood flow in breast tumors." Opt. Lett., 2005, 30(21): 29152917.

60. Liebert A, Wabnitz H, Steinbrink J, Moller M, Macdonald R, Rinneberg H, Villringer A, Obrig H, "Bed-side assessment of cerebral perfusion in stroke patients based on optical monitoring of a dye bolus by time-resolved diffuse reflectance." NeuroImage, 2005, 24: 426-435.

61. Sunar U, Quon H, Durduran T, Zhang J, Du J, Zhou C, Yu G, Choe R, Kilger A, Lustig R, Loevner L, Nioka S, Chance B, Yodh AG, "Noninvasive diffuse optical measurement of blood flow and blood oxygenation for monitoring radiation therapy in patients with head and neck tumors: a pilot study." J Biomed Opt, 2006, 11(6): 064021.

62. Zhu Q, Durduran T, Ntziachristos V, Holboke M, Yodh AG, "Imager that combines near-infrared diffusive light and ultrasound,” Opt. Lett., 1999, 24(15), 1050-1052.

63. Guo P, Piao D, Zhu Q, Fikiet J, "A combined 2-D ultrasound and NIR imaging system." Proc. of the IEEE 26th Annual NE Bioeng. ConferencE, April 8-9, 2000, 7778.

64. Zhu Q, Chen NG, Guo P, Yan SK, Piao D, "Combined ultrasound and near infrared diffusive light imaging." IEEE Symposium on Ultrasonics, 2000, 1629-1632.

65. Chen NG, Guo P, Yan S, Piao D, Zhu Q, "Simultaneous near-infrared diffusive light and ultrasound imaging." Appl. Opt., 2001, 40(34): 6367-6380.

66. Zhu Q, Chen NG, Kurtzman SH, "Imaging tumor angiogenesis by use of combined near-infrared diffusive light and ultrasound." Optics Letters, 2003, 28(5): 337-339.

67. Zhu Q, Huang M, Chen NG, Zarfos K, Jagjivan B, Kane M, Hedge P, Kurtzman SH, "Ultrasound-guided optical tomographic imaging of malignant and benign breast lesions: initial clinical results of 19 cases." Neoplasia, 2003, 5(5): 379-388.

68. Chen NG, Huang M, Xia H, Piao D, "Portable near-infrared diffusive light imager for breast cancer detection.” J Biomed Opt, 2004, 9(3): 504-510.

69. Zhu Q, Cronin EB, Currier AA, Vine HS, Huang M, Chen NG, Xu C, "Benign versus malignant breast masses: optical differentiation with US-guided optical imaging reconstruction." Radiology, 2005, 237: 57-66.

70. Zhu Q, Kurtzman SH, Hegde P, Tannenbaum S, Kane M, Huang M, Chen NG, Jagivan B, Zarfos K, "Utilizing optical tomography with ultrasound localization to image heterogeneous hemoglobin distribution in large breast cancers." Neoplasia, 2005, 7(3): 263-270. 
71. Xu C, Zhu Q, "Optimal probe design for dual-modality breast imaging." Proc. of SPIE - Optical Tomography and Spectroscopy of Tissue VII, Britton Chance, Robert R. Alfano, Bruce J. Tromberg, Mamoru Tamura, Eva M. Sevick-Muraca, eds February 13, 2007, 6434: 64340B.

72. Chance B, Leigh JS, Miyake H, Smith DS, Nioka S, Greenfeld R, Finander M, Kaufmann K, Levy W, Young M, Cohen P, Yoshioka H, Borestsky R, "Comparison of time-resolved and -unresolved measurements of deoxyhemoglobin in brain." Proc. Natl. Acad. Sci. USA, 1988, 85: 4791-4975.

73. Doornbos RMP, Lang R, Aalders MC, Cross FW, Sterenborg HJCM, "The determination of in vivo human tissue optical properties and absolute chromophore concentrations using spatially resolved steady-state diffuse reflectance spectroscopy." Phys. Med. Biol., 1999, 44: 967-981.

74. Arridge SR, Lionheart WRB, "Nonuniqueness in diffusion-based optical tomography." Opt. Lett., 1998, 23: 882-884.

75. Balgi G, Reynolds JS, Mayer RH, Cooley R, Sevick-Muraca EM, "Measurements of multiply scattered light for on-line monitoring of changes in size distribution of celldebris suspensions.' Biotechnol. Prog., 1999, 15: 1106-1114.

76. Schmitt JM, Knuttel A, Knutson JR, "Interference of diffusive light waves." J. Opt. Soc. Am. A., 1992, 9: 1832-1843.

77. Knuttel A, Schmitt JM, Barnes R, Knutson JR, "Spatial localization of absorbing bodies by interfering diffusive photon density waves." Appl. Opt., 1993, 32: 381-389.

78. Knuttel A, Schmitt JM, Barnes R, Knutson JR. "Acoustic-optic scanning and interfering photon density waves for precise localization of an absorbing (or fluorescent) body in a turbid medium." Rev. Sci. Instrum., 1993, 64: 638-644.

79. Chance B, Kang K, He L, Weng J, Sevick E, "Highly sensitive object location in tissue models with linear in-phase and anti-phase multi-element optical arrays in one and two dimensions." Proc. Natl. Acad. Sci. USA, 1993, 90: 3423-3427.

80. Chen $\mathrm{Y}, \mathrm{Mu} \mathrm{C}$, Intes $\mathrm{X}$, Chance B, "Adaptive calibration for object localization in turbid media with interfering diffuse photon density waves.” Appl. Opt., 2002, 41: 7325-7333.

81. Wu J, Wang Y, Perelman L, Itzkan I, Dasari R R and Feld M S 1995 Time-resolved multichannel imaging of fluorescent objects embedded in turbid media Opt. Lett. 20 489-91.

82. Wu J, Perelman L, Dasari R R and Feld M S 1997 Fluorescence tomographic imaging in turbid media using early-arriving photons and Laplace transforms Proc. Natl. Acad. Sci. 94 8783-8. 
83. Chang J, Graber H L and L. B R 1997 Imaging of fluorescence in highly scattering media IEEE Transactions on Biomedical Engineering 44 810-22.

84. Hull E L, Nichols M G and Foster T H 1998 Localization of luminescent inhomogeneities in turbid media with spatially resolved measurements of $\mathrm{cw}$ diffuse luminescence emittance Appl. Opt. 37.

85. Chernomordik V, Hattery D, Gannot I and Gandjbakhche A H 1999 Inverse method 3-D reconstruction of localized in vivo fluorescence - application to Sjogren syndrome IEEE J. on Selected Topics on Quantum Electronics 54 930-5.

86. Hawrysz D J, Eppstein M J, Lee J and Sevick-Muraca E M 2001 Error consideration in contrast-enhanced three-dimensional optical tomography, Opt. Lett. 26 704-6.

87. Eppstein M J, Hawrysz D J, Godavarty A and Sevick-Muraca E M 2002 Threedimensional, Bayesian image reconstruction from sparse and noisy data sets: Nearinfrared fluorescence tomography Proc. Natl. Acad. Sci. 99 9619-9624.

88. Ntziachristos V and Weissleder R 2001 Experimental three-dimensional fluorescence reconstruction of diffuse media by use of a normalized Born approximation Opt. Lett. 26 893-895.

89. Ntziachristos V, Bremer C, Tung C and Weissleder R 2002a Imaging cathepsin B upregulation in HT-1080 tumor models using fluorescence-mediated molecular tomography (FMT) Acad. Radiol. 9(suppl 2) S323-S325.

90. Lee J and Sevick-Muraca E 2001 Fluorescence-enhanced absorption imaging using frequency-domain photon migration: tolerance to measurement error Journal of Biomedical Optics 6 58-67.

91. Lee J and Sevick-Muraca E M 2002 Three-dimensional fluorescence enhanced optical tomography using referenced frequency-domain photon migration measurements at emission and excitation wavelengths $J$ Opt Soc Am A 19 759-71

92. Graves E E, Ripoll J, Weissleder R and Ntziachristos V 2003 A submillimeter resolution fluorescence molecular imaging system for small animal imaging Med. Phys. 30 901-11.

93. Godavarty A, Eppstein M J, Zhang C, Theru S, Thompson A B, Gurfinkel M and Sevick-Muraca E M 2003 Fluorescence-enhanced optical imaging in large tissue volumes using a gain-modulated ICCD camera Phys. Med. Biol. 48 1701-20.

94. Godavarty A, Thompson A B, Roy R, Gurfinkel M, Eppstein M J, Zhang C and Sevick-Muraca E M 2004a Diagnostic imaging of breast cancer using fluorescenceenhanced optical tomography: phantom studies $J$ Biomed Opt special edition on Biomedical Optics and Women's Health 9(3) 488-496. 
95. Godavarty A, Zhang C, Eppstein M J and Sevick-Muraca E M 2004b Fluorescenceenhanced optical imaging of large phantoms using single and simultaneous dual point illumination geometries Med. Phys. 31 183-90.

96. Klose A D, Ntziachristos V and Hielscher A H 2005 The inverse source problem based on the radiative transfer equation in optical molecular imaging J. Compu. Phys. $202323-45$.

97. Wu C, Barnhill H, Liang X, Wang Q and Jiang H 2005a A new probe using hybrid virus-dye nanoparticles for near-infrared fluorescence tomography Opt. Commu. 255 366-74.

98. Wu C, Liang X and Jiang H 2005b Metal nanoshells as a contrast agent in near-infrared diffuse optical tomography Opt. Commu. 253 214-21.

99. Roy R, Thompson A B, Godavarty A and Sevick-Muraca E M 2005 Tomographic fluorescence imaging in tissue phantoms: A novel reconstruction algorithm and imaging geometry Ieee Transactions On Medical Imaging 24 137-54.

100. Godavarty A, Sevick-Muraca E M and Eppstein M J 2005 Three-dimensional fluorescence lifetime tomography Med. Phys. 32 992-1000.

101. Chen Y, Intes X and Chance B 2005 Development of high-sensitivity near-infrared fluorescence imaging device for early cancer detection Biomed Instrum Technol 39 75-85.

102. Patwardhan S V, Bloch S R, Achilefu S and Culver J P 2005 Time-dependent wholebody fluorescence tomography of probe bio-distributions in mice Opt. Exp. 13 256477.

103. Zacharakis G, Ripoll J, Weissleder R and Ntziachristos V 2005 Fluorescent protein tomography scanner for small animal imaging IEEE Trans. Med. Imaging 24 878-85.

104. Fedele F, Laible J P and Eppstein M J 2003 Coupled complex adjoint sensitivities for frequency-domain fluorescence tomography: Theory and vectorized implementation $J$. Comput. Phys. 187(2) 597-619.

105. Joshi A, Bangerth W, Hwang K, Rasmussen J C and Sevick-Muraca E M 2006a Fully adaptive FEM based fluorescence optical tomography from time-dependent measurements with area illumination and detection Med. Phys. 33(5) 1299-1310.

106. Roy Ranadhir, Godavarty Anuradha and Sevick-Muraca Eva M 2006 Fluorescenceenhanced optical tomography of a large tissue phantom using point illumination geometries Journal of Biomedical Optics 11(4) 044007 044001-044017.

107. Schulz RB, Peter J, Semmler W, D'Andrea C, Valentini G and Cubeddu R 2006 Comparison of noncontact and fiber-based fluorescence-mediated tomography $\mathrm{Opt}$ Lett. Mar 15; 31(6):769-71. 
108. Yuan B and Zhu Q 2006 Separately reconstructing the structural and functional parameters of a fluorescent inclusion embedded in a turbid medium OPTICS EXPRESS 14(16) 7172-7187.

109. Mohajerani P, Eftekhar A A, Huang J, and Adibi A 2007 "Optimal sparse solution for fluorescent diffuse optical tomography: theory and phantom experimental results," Appl. Opt. 46, 1679-1685.

110. Corlu A, Choe R, Durduran T, Rosen M A, Schweiger M, Arridge S R, Schnall, Mitchell M D, and Yodh A G 2007 Three-dimensional in vivo fluorescence diffuse optical tomography of breast cancer in humans Opt. Exp. 15(11) 6696-6716.

111. Davis S C, Hamid D, Wang J, Jiang S, Pogue B W and Paulsen K D 2007 Imageguided diffuse optical fluorescence tomography implemented with Laplacian-type regularization Opt. Exp. 15(7) 4066-4082.

112. Deliolanis N, Lasser T, Hyde D, Soubret A, Ripoll J and Ntziachristos V. 2007 Freespace fluorescence molecular tomography utilizing 360 degrees geometry projections Opt Lett. Feb 15;32(4):382-4.

113. Herve A K L, Silva A Da, Berger M, Boetet J, Dinten J M, Peltie P and Rizo P 2007 Noncontact flouorecence diffuse optical tomography of heterogenous media App. Opt. 46(22) 4896-4906.

114. Kepshire D S, Davis S C, Dehghani H, Paulsen K D and Pogue B W 2007b Subsurface diffuse optical tomography can localize absorber and fluorescent objects but recovered image sensitivity is nonlinear with depth Appl. Opt. 46(10) 1669-1678.

115. Kepshire D S, Davis S C, Dehghani H, Paulsen KD and Pogue BW 2008 Fluorescence tomography characterization for sub-surface imaging with protoporphyrin IX Opt Express. Jun 9;16(12):8581-93.

116. Montet X, Figueiredo JL, Alencar H, Ntziachristos V, Mahmood U and Weissleder R 2007 Tomographic fluorescence imaging of tumor vascular volume in mice Radiology Mar 242(3):751-8.

117. Meyer H, Garofalakis A, Zacharakis G, Psycharakis S, Mamalaki C, Kioussis D, Economou EN, Ntziachristos V and Ripoll J 2007 Noncontact optical imaging in mice with full angular coverage and automatic surface extraction Appl Opt. 1046 (17): 361727.

118. Roy Ranadhir, Godavarty Anuradha and Sevick-Muraca Eva M 2007 Fluorescenceenhanced three-dimensional lifetime imaging: a phantom study Physics and medicine in biology 52(2007) 4155-4170. 
119. Keren S, Gheysens O, Levin CS and Gambhir SS 2008 A comparison between a time domain and continuous wave small animal optical imaging system IEEE Trans Med Imaging. Jan 27(1):58-63.

120. Tan Y and Jiang H. 2008 Diffuse optical tomography guided quantitative fluorescence molecular tomography Appl Opt. Apr 20;47(12):2011-6.

121. Davis S C, Pogue B W, Springett R, Leussler C, Mazurkewitz P, Tuttle S B, GibbsStrauss S L, Jiang S S, Dehghani H, and Paulsen K D 2008 Magnetic resonancecoupled fluorescence tomography scanner for molecular imaging of tissue Rev. Sci. Inst. 79, 064302 .

122. Mohajerani P, Adibi A, Kempner J, and Yared W 2009 Compensation of optical heterogeneity-induced artifacts in fluorescence molecular tomography: theory and in vivo validation J. Biomed. Opt. 14(3), 034021.

123. Davis S C, Pogue B W, Tuttle S B, Dehghani H, and Paulsen K D 2009 Spectral distortion in diffuse molecular luminescence tomography in turbid media J. Appl. Phys. 105, 102024.

124. Ziegler R, Nielsen T, Koehler T, Grosenick D, Steinkellner O, Hagen A, Macdonald R, and Rinneberg H 2009 Appl. Opt. 48(24) 4651-4662.

125. Deliolanis N C, Dunham J, Wurdinger T, Figueiredo J L, Tannous, B A and Ntziachristos V 2009 JBO Lett. 14(3) 030509.

126. Kepshire D, Mincu N, Hutchins M, Gruber J, Dehghani H, Hypnarowski J, Leblond F, Khayat M, and Pogue B W 2009 Rev. Sci. inst. 80, 043701.

127. Ge J, Erickson S J, and Godavarty A 2009 Fluorescence tomographic imaging using a hand-held probe based optical imager: extensive phantom studies Appl. OpT. 48(33), 6408-6416.

128. Lin Y, Barber W C, Iwanczyk J S, Roeck W, Nalcioglu O, and Gulsen G 2010 Quantitative fluorescence tomography using a combined tri-modality FT/DOT/XCT system Opt. Exp. 18(8) 7835-7850.

129. Hyde D, Miller E L, Brooks D H, and Ntziachristos V 2010 Data Specific Spatially Varying Regularization for Multimodal Fluorescence Molecular Tomography IEEE Trans. Med. Imag. 29(2) 365-374.

130. Schulz R B, Ale A, Sarantopoulos A, Freyer M, Soehngen E, Zientkowska M, and Ntziachristos V 2010 IEEE Trans. Med. Imag. 29(2) 465-473.

131. Liu X, Wang D, Liu F, and Bai J 2010 Principal component analysis of dynamic fluorescence diffuse optical tomography images Opt. Exp. 18(6) 6300-6314. 
132. Baritaux J C, Hassler K, and Unser M 2010 An Efficient Numerical Method for General Lp Regularization in Fluorescence Molecular Tomography IEEE Trans. Med. Imag.29(4) 1075-1087.

133. Biswal N C, Gamelin J K, Yuan B, Backer M V, Backer J M, Zhu Q 2010 Fluorescence imaging of vascular endothelial growth factor in tumors for mice embedded in a turbid medium J. Biomed. Opt. 15(1) 016012.

134. Leff D R, Warren O, Enfield L C, Gibson A, Athanasiou T, Patten D K, Hebden J, Yang G Z, Darzi A 2008 Diffuse optical imaging of the healthy and diseased breast: a systematic review Breast Cancer Res. Treat. , 2008, 108:9-22.

135. Grosenick D, Moesta KT, Wabnitz H et al 2003 Time-domain optical mammography: initial clinical results on detection and characterization of breast tumors. Appl Opt 42:3170-3186.

136. Intes X 2005 Time-domain optical mammography SoftScan: initial results. Acad Radiol 12:934-947.

137. Yates T, Hebden JC, Gibson AP et al 2005 Optical tomography of the breast using a multi-channel time-resolved imager. Phys Med Biol 50:2503-2517.

138. Taroni P, Danesini G, Torricelli A et al 2004 Clinical trial of time-resolved scanning optical mammography at 4 wavelengths between 683 and $975 \mathrm{~nm}$. J Biomed Opt 9:464-473.

139. Suzuki K Yamashita Y, Ohta K et al 1996 Quantitative measurements of optical parameters in normal breasts using timeresolved spectrsocopy: in vivo results of 30 Japanese women. J Biomed Opt 1:330-334.

140. Taroni P, Torricelli A, Spinelli L et al (2005) Time-resolved optical mammography between 637 and $985 \mathrm{~nm}$ : clinical study on the detection and identification of breast lesions. Phys Med Biol 50:2469-2488.

141. Grosenick D, Wabnitz H, Moesta KT et al (2005) Time-domain scanning optical mammography: II. Optical properties and tissue parameters of 87 carcinomas. Phys Med Biol 50:2451-2468.

142. Durduran T, Choe R, Culver JP et al (2002) Bulk optical properties of healthy female breast tissue. Phys Med Biol 47: 2847-2861.

143. Spinelli L, Torricelli A, Pifferi A et al (2004) Bulk optical properties and tissue components in the female breast from multiwavelength time-resolved optical mammography. J Biomed Opt 9:1137-114.

144. Pogue BW, Jiang S, Dehghani H et al (2004) Characterization of hemoglobin, water, and NIR scattering in breast tissue: analysis of intersubject variability and menstrual cycle changes. J Biomed Opt 9:541-552. 
145. Poplack SP, Paulsen KD, Hartov A et al (2004) Electromagnetic breast imaging: average tissue property values in women with negative clinical findings. Radiology 231:571-580.

146. You S S, Jiang Y X, Zhu Q L, Liu J B, Zhang J, Dai Q, Liu H, Sun Q 2010 US-guided diffuse optical tomography: a promising functional imaging technique in breast lesions Eur. Radiol. 20, 309-317.

147. Soliman H, Gunasekara A, Rycroft M, Zubovits J, Dent R, Spatne J, Yaffe M J, Czarnota G J 2010 Functional imaging using diffuse optical spectroscopy of neoadjuvant chemotherapy response in women with locally advanced breast cancer Clin. Can. Res. 16(9) 2605-2614.

148. Choe R, Konecky S D, Corlu A, Lee K, Durduran T, Busch D R, Pathak S, Czerniecki B J, Tchou J, Fraker D L 2009 Differentiation of benign and malignant breast tumors by in-vivo three-dimensional parallel-plate diffuse optical tomography J. Biomed. Opt. 14(2) 024020 .

149. Jiang S, Pogue B, Carpenter C M, Poplack S P, Wells W A, Kogel C A, Forero J A, Muffly L S, Schwartz G N, Paulsen K D, Kaufman P A 2009 Evaluation of Breast Tumor Response to Neoadjuvant Chemotherapy with Tomographic Diffuse Optical Spectroscopy: Case Studies of Tumor Region-of-Interest Changes Radiol. 252(2) 551560.

150. Kukreti S, Cerussi A E, Tanamai W, Hsiang D, Tromberg B J, Gratton E 2010 Characterization of metabolic differences between benign and malignant tumors: High spectral resolution diffuse optical spectroscopy Radiol. 254(1) 277-284.

151. Stahel M C, Wolf M, Baños A, Hornung R 2009 Optical properties of the breast during spontaneous and birth control pill-mediated menstrual cycles Lasers. Med. Sci. 24, 901-907.

152. van de Ven S, Wiethoff A, Nielsen T, Brendel B, van der VoortM, Nachabe R, Van der Mark M, Van Beek M, Bakker L, Fels L, Elias S, Luijten P, Mali W 2009 A Novel Fluorescent Imaging Agent for Diffuse Optical Tomography of the Breast: First Clinical Experience in Patients Mol. Imag. Biol. 12, 343-348.

153. Regalado S, Erickson S J, Zhu B, Ge J, and Godavarty A 2010 Automated coregistered imaging using a hand-held probe-based optical imager Rev. Sci. Inst. 81: 023702.

154. Ge J 2008 Fluorescence-enhanced optical imaging on 3D phantoms using a hand-held probe based frequency-domain intensified charge coupled device optical imager, Ph.D. Dissertation, Florida International University. 
155. Erickson S J, Martinez S L, Gonzalez J, Caldera L, and Godavarty A 2010 Improved detection limits using a hand-held optical imager with coregistration capabilities Biomed. Opt. Exp. 1, 126-134.

156. Erickson S J, Ge J, Sanchez A, and Godavarty A 2010 Two-dimensional fast surface imaging using a hand-held optical device: in-vitro and in-vivo fluorescence studies Trans. Onc. 3(1): 16-22. 
APPENDICES 


\section{APPENDIX A -- Complete Table of Hand-held Based Optical Imaging Devices}




\begin{tabular}{|c|c|c|c|c|c|c|c|c|c|c|c|}
\hline & $\begin{array}{l}\text { Refere } \\
\text { nce }\end{array}$ & Modality & $\begin{array}{l}\text { Meas. } \\
\text { Techniq } \\
\text { ue }\end{array}$ & Source Type & $\begin{array}{c}\text { Source } \\
\text { Wavelengt } \\
\text { h }\end{array}$ & $\begin{array}{c}\text { Detector } \\
\text { Type }\end{array}$ & $\begin{array}{l}\text { Target } \\
\text { Depth }\end{array}$ & $\begin{array}{l}\text { Subject } \\
\text { Studied }\end{array}$ & $\begin{array}{c}\# \\
\text { Sources, } \\
\text { Detector } \\
\text { s }\end{array}$ & \multicolumn{2}{|l|}{ Picture / Schematic } \\
\hline \multirow{7}{*}{ 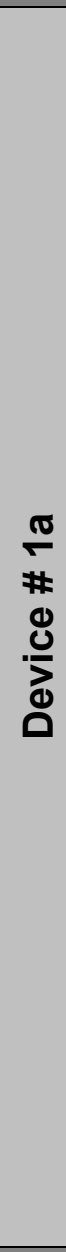 } & $\begin{array}{c}\text { Tromb } \\
\text { erg, } \\
1997 \\
{[26]}\end{array}$ & $\begin{array}{c}\text { NIR } \\
\text { Spectrosc } \\
\text { opy }\end{array}$ & $\begin{array}{c}\text { FDPM } \\
(300 \mathrm{kHz} \\
-1 \mathrm{GHz})\end{array}$ & $\begin{array}{l}\text { amplitude } \\
\text { modulated } \\
\text { diode lasers } \\
(\mathbf{1 0 - 3 0 ~} \mathbf{m W})\end{array}$ & $\begin{array}{c}674,811 \\
849,956 \\
\mathrm{~nm}\end{array}$ & APD & $1.0 \mathrm{~cm}$ & $\begin{array}{c}2 \\
\text { abnormal } \\
\text { human } \\
\text { subjects } \\
\text { (breast } \\
\text { tissue) }\end{array}$ & 1,1 & \multirow{7}{*}{\multicolumn{2}{|c|}{$2.2 \mathrm{~cm}$}} \\
\hline & $\begin{array}{c}\text { Lannin } \\
\text { g, } \\
1999 \\
{[27]}\end{array}$ & $\begin{array}{c}\text { NIR } \\
\text { Spectrosc } \\
\text { opy }\end{array}$ & $\begin{array}{c}\text { FDPM } \\
(5 \mathrm{MHz}- \\
1 \mathrm{GHz})\end{array}$ & $\begin{array}{l}\text { amplitude } \\
\text { modulated } \\
\text { diode lasers }\end{array}$ & $\begin{array}{c}674,803 \\
849,956 \\
\mathrm{~nm}\end{array}$ & APD & $\begin{array}{l}1.26 \\
\mathrm{~cm}\end{array}$ & $\begin{array}{c}4 \\
\text { abnormal } \\
\text { human } \\
\text { subjects } \\
\text { (breast } \\
\text { tissue) }\end{array}$ & 1,2 & & \\
\hline & $\begin{array}{l}\text { Tromb } \\
\text { erg, } \\
2000 \\
{[28]}\end{array}$ & $\begin{array}{c}\text { NIR } \\
\text { Spectrosc } \\
\text { opy }\end{array}$ & $\begin{array}{c}\text { FDPM } \\
(300 \mathrm{kHz} \\
-1 \mathrm{GHz})\end{array}$ & $\begin{array}{l}\text { amplitude } \\
\text { modulated } \\
\text { diode lasers } \\
(\mathbf{1 0 - 3 0 ~} \mathrm{mW})\end{array}$ & $\begin{array}{c}674,811 \\
849,956 \\
\mathrm{~nm}\end{array}$ & APD & $\begin{array}{l}0.74 \\
\mathrm{~cm}\end{array}$ & $\begin{array}{c}4 \text { normal } \\
\& 3 \\
\text { abnormal } \\
\text { human } \\
\text { subjects } \\
\text { (breast } \\
\text { tissue) }\end{array}$ & 1,1 & & \\
\hline & $\begin{array}{l}\text { Holbok } \\
\text { e, } \\
2000 \\
{[29]}\end{array}$ & $\begin{array}{c}\text { NIR } \\
\text { Spectrosc } \\
\text { opy (w/ } \\
\text { ultrasoun } \\
\text { d } \\
\text { constraint } \\
\text { s) }\end{array}$ & $\begin{array}{l}\text { FDPM } \\
(400 \\
\mathrm{MHz})\end{array}$ & $\begin{array}{l}\text { diode lasers } \\
(10-30 \mathrm{~mW})\end{array}$ & $\begin{array}{c}674,782 \\
803,849 \\
\mathrm{~nm}\end{array}$ & APD & $\begin{array}{l}0.74 \\
\mathrm{~cm}\end{array}$ & $\begin{array}{c}1 \\
\text { abnormal } \\
\text { human } \\
\text { subject } \\
\text { (breast } \\
\text { tissue) }\end{array}$ & 1,1 & & \\
\hline & $\begin{array}{c}\text { Ceruss } \\
\mathrm{i}, 2001 \\
{[30]}\end{array}$ & $\begin{array}{c}\text { NIR } \\
\text { Spectrosc } \\
\text { opy }\end{array}$ & $\begin{array}{l}\text { FDPM } \\
(1 \mathrm{GHz})\end{array}$ & $\begin{array}{l}\text { amplitude } \\
\text { modulated } \\
\text { diode lasers } \\
(5-25 \mathrm{~mW})\end{array}$ & $\begin{array}{c}672,800 \\
806,852, \\
896,913 \\
978 \mathrm{~nm}\end{array}$ & APD & $1.0 \mathrm{~cm}$ & $\begin{array}{c}28 \text { normal } \\
\text { human } \\
\text { subjects } \\
\text { (breast } \\
\text { tissue) } \\
\end{array}$ & 1,1 & & \\
\hline & $\begin{array}{l}\text { Shah, } \\
2001 \\
{[31]}\end{array}$ & $\begin{array}{c}\text { NIR } \\
\text { Spectrosc } \\
\text { opy }\end{array}$ & $\begin{array}{c}\text { FDPM } \\
(50-1000 \\
\mathrm{MHz})\end{array}$ & $\begin{array}{l}\text { amplitude } \\
\text { modulated } \\
\text { diode lasers } \\
(\mathbf{1 0 - 2 0 ~} \mathbf{~ W W})\end{array}$ & $\begin{array}{c}674,803 \\
849,894 \\
947 \\
956 / 980 \mathrm{~nm}\end{array}$ & APD & $N / A$ & $\begin{array}{c}14 \text { normal } \\
\text { human } \\
\text { subjects } \\
\text { (breast } \\
\text { tissue) } \\
\end{array}$ & 1,1 & & \\
\hline & $\begin{array}{l}\text { Shah, } \\
2004 \\
{[32]}\end{array}$ & $\begin{array}{c}\text { NIR } \\
\text { Spectrosc } \\
\text { opy }\end{array}$ & $\begin{array}{c}\text { FDPM } \\
(50-700 \\
\mathrm{MHz})\end{array}$ & $\begin{array}{l}\text { amplitude } \\
\text { modulated } \\
\text { diode lasers } \\
(5-25 \mathrm{~mW})\end{array}$ & $\begin{array}{c}674,780 \\
803,849 \\
894,915 \\
980 \mathrm{~nm}\end{array}$ & APD & N/A & $\begin{array}{c}31 \text { normal } \\
\text { human } \\
\text { subjects } \\
\text { (breast } \\
\text { tissue) }\end{array}$ & 1,1 & & \\
\hline & & & & & & & & & & & \\
\hline
\end{tabular}




\begin{tabular}{|c|c|c|c|c|c|c|c|c|c|}
\hline \multirow{6}{*}{$\begin{array}{l}\text { 을 } \\
\# \\
0 \\
0 \\
0\end{array}$} & $\begin{array}{l}\text { Bevilac } \\
\text { qua, } \\
2000 \\
{[33]}\end{array}$ & $\begin{array}{c}\text { NIR } \\
\text { Spectrosc } \\
\text { opy }\end{array}$ & $\begin{array}{c}\text { CW \& } \\
\text { FDPM } \\
(100-700 \\
\mathrm{MHz})\end{array}$ & $\begin{array}{c}\text { FD - amplitude } \\
\text { modulated } \\
\text { diode lasers } \\
(<20 \mathrm{~mW}) \\
\mathbf{C W}-150 \mathrm{~W} \\
\text { halogen lamp }\end{array}$ & $\begin{array}{c}672,800, \\
806,852, \\
896,913, \\
978 \mathrm{~nm}\end{array}$ & $\begin{array}{c}\text { FD - APD } \\
\text { CW - fiber- } \\
\text { coupled } \\
\text { spectrogra } \\
\text { ph wl } \\
\text { linear } \\
\text { CCD } \\
\text { detector }\end{array}$ & $\mathrm{N} / \mathrm{A}$ & $\begin{array}{c}\text { phantom } \\
\& 2 \\
\text { normal } \\
\text { human } \\
\text { subjects } \\
\text { (breast } \\
\text { tissue) }\end{array}$ & $\begin{array}{c}2,2 \\
\text { (sequenti } \\
\text { al } \\
\text { illuminatio } \\
\mathrm{n} \text { ) }\end{array}$ \\
\hline & $\begin{array}{c}\text { Ceruss } \\
\text { i, 2002 } \\
{[34]}\end{array}$ & $\begin{array}{c}\text { NIR } \\
\text { Spectrosc } \\
\text { opy }\end{array}$ & $\begin{array}{c}\text { CW \& } \\
\text { FDPM } \\
(300 \mathrm{kHz} \\
-1 \mathrm{GHz}) \\
{[2} \\
\text { separate } \\
\text { probes] }\end{array}$ & $\begin{array}{c}\text { FD - } 7 \text { diode } \\
\text { lasers } \\
\mathbf{2 5} \mathbf{~ m W )} \\
\mathbf{C W}-150 \mathrm{~W} \\
\text { halogen lamp }\end{array}$ & $\begin{array}{c}672,800, \\
806,852, \\
896,913, \\
978 \mathrm{~nm}\end{array}$ & $\begin{array}{l}\text { FD - APD } \\
\text { CW - fiber- } \\
\text { coupled } \\
\text { spectrogra } \\
\text { ph w/ } \\
\text { linear } \\
\text { CCD } \\
\text { detector }\end{array}$ & $\begin{array}{c}1.0 \mathrm{~cm} \\
\text { (averag } \\
\text { e) }\end{array}$ & $\begin{array}{c}30 \text { normal } \\
\& 1 \\
\text { abnormal } \\
\text { human } \\
\text { subjects } \\
\text { (breast } \\
\text { tissue) }\end{array}$ & 1,1 \\
\hline & $\begin{array}{l}\text { Jakubo } \\
\text { wski, } \\
2004 \\
{[35]}\end{array}$ & $\begin{array}{c}\text { NIR } \\
\text { Spectrosc } \\
\text { opy }\end{array}$ & $\begin{array}{c}\text { CW \& } \\
\text { FDPM } \\
(50-1000 \\
\mathrm{MHz})\end{array}$ & $\begin{array}{c}\text { FD - amplitude } \\
\text { modulated } \\
\text { diode lasers } \\
\text { CW - high- } \\
\text { intensity } \\
\text { tungsten } \\
\text { halogen }\end{array}$ & $\begin{array}{c}660,685, \\
786,809, \\
822,852, \\
898,911, \\
946,973 \\
\text { nm }\end{array}$ & $\begin{array}{l}\text { FD - APD } \\
\text { CW - fiber- } \\
\text { coupled } \\
\text { spectrogra } \\
\text { ph w/ } \\
\text { linear } \\
\text { CCD } \\
\text { detector }\end{array}$ & $\mathrm{N} / \mathrm{A}$ & $\begin{array}{c}1 \\
\text { abnormal } \\
\text { human } \\
\text { subject } \\
\text { (breast } \\
\text { tissue) }\end{array}$ & $\begin{array}{c}2,2 \\
\text { (sequenti } \\
\text { al } \\
\text { illuminatio } \\
\mathrm{n} \text { ) }\end{array}$ \\
\hline & $\begin{array}{l}\text { Shah, } \\
2005 \\
{[36]}\end{array}$ & $\begin{array}{c}\text { NIR } \\
\text { Spectrosc } \\
\text { opy }\end{array}$ & $\begin{array}{c}\text { CW \& } \\
\text { FDPM } \\
(50-600 \\
\mathrm{MHz})\end{array}$ & $\begin{array}{c}\text { FD - amplitude } \\
\text { modulated } \\
\text { diode lasers } \\
\text { CW - high- } \\
\text { intensity } \\
\text { tungsten } \\
\text { halogen }\end{array}$ & $\begin{array}{c}658,682 \\
785,810 \\
830,850 \\
n m\end{array}$ & $\begin{array}{c}\text { FD - APD } \\
\text { CW - fiber- } \\
\text { coupled } \\
\text { spectrogra } \\
\text { ph w/ } \\
\text { linear } \\
\text { CCD } \\
\text { detector }\end{array}$ & $\begin{array}{l}0.5 \mathrm{~cm} \\
\text { (averag } \\
\text { e) }\end{array}$ & $\begin{array}{c}1 \\
\text { abnormal } \\
\text { human } \\
\text { subject } \\
\text { (breast } \\
\text { tissue) }\end{array}$ & $\begin{array}{c}2,2 \\
\text { (sequenti } \\
\text { al } \\
\text { illuminatio } \\
n \text { ) }\end{array}$ \\
\hline & $\begin{array}{l}\text { Hsiang } \\
, 2005 \\
{[37]}\end{array}$ & $\begin{array}{c}\text { NIR } \\
\text { Spectrosc } \\
\text { opy } \\
\text { (w/ MRI } \\
\text { coregistra } \\
\text { tion) }\end{array}$ & $\begin{array}{c}\text { CW \& } \\
\text { FDPM } \\
(50-600 \\
\text { MHz) }\end{array}$ & $\begin{array}{c}\text { FD - amplitude } \\
\text { modulated } \\
\text { diode lasers } \\
(\mathbf{1 0 - 2 0} \mathbf{~ m W )} \\
\mathbf{C W}-\text { high- } \\
\text { intensity } \\
\text { tungsten } \\
\text { halogen }\end{array}$ & $\begin{array}{c}658,682 \\
785,810 \\
830,850 \\
n m\end{array}$ & $\begin{array}{c}\text { FD - APD } \\
\text { CW - fiber- } \\
\text { coupled } \\
\text { spectrogra } \\
\text { ph w/ } \\
\text { linear } \\
\text { CCD } \\
\text { detector }\end{array}$ & $4.2 \mathrm{~cm}$ & $\begin{array}{c}6 \\
\text { abnormal } \\
\text { human } \\
\text { subjects } \\
\text { (breast } \\
\text { tissue) }\end{array}$ & $\begin{array}{c}2,2 \\
\text { (sequenti } \\
\text { al } \\
\text { illuminatio } \\
\mathrm{n} \text { ) }\end{array}$ \\
\hline & $\begin{array}{c}\text { Ceruss } \\
\text { i, 2006 } \\
{[38]}\end{array}$ & $\begin{array}{c}\text { NIR } \\
\text { Spectrosc } \\
\text { opy }\end{array}$ & $\begin{array}{c}\text { CW \& } \\
\text { FDPM } \\
(50-500 \\
\mathrm{MHz})\end{array}$ & $\begin{array}{c}\text { FD - amplitude } \\
\text { modulated } \\
\text { diode lasers } \\
(<20 \mathrm{~mW}) \\
\mathbf{C W}-150 \mathrm{~W} \\
\text { halogen lamp }\end{array}$ & $\begin{array}{c}661,686, \\
786,808, \\
822,852 \\
n m\end{array}$ & $\begin{array}{l}\text { FD - APD } \\
\text { CW - fiber- } \\
\text { coupled } \\
\text { spectrogra } \\
\text { ph w/ } \\
\text { linear } \\
\text { CCD } \\
\text { detector }\end{array}$ & $\mathrm{N} / \mathrm{A}$ & $\begin{array}{c}57 \\
\text { abnormal } \\
\text { human } \\
\text { subjects } \\
\text { (breast } \\
\text { tissue) }\end{array}$ & $\begin{array}{c}2,2 \\
\text { (sequenti } \\
\text { al } \\
\text { illuminatio } \\
\text { n) }\end{array}$ \\
\hline
\end{tabular}
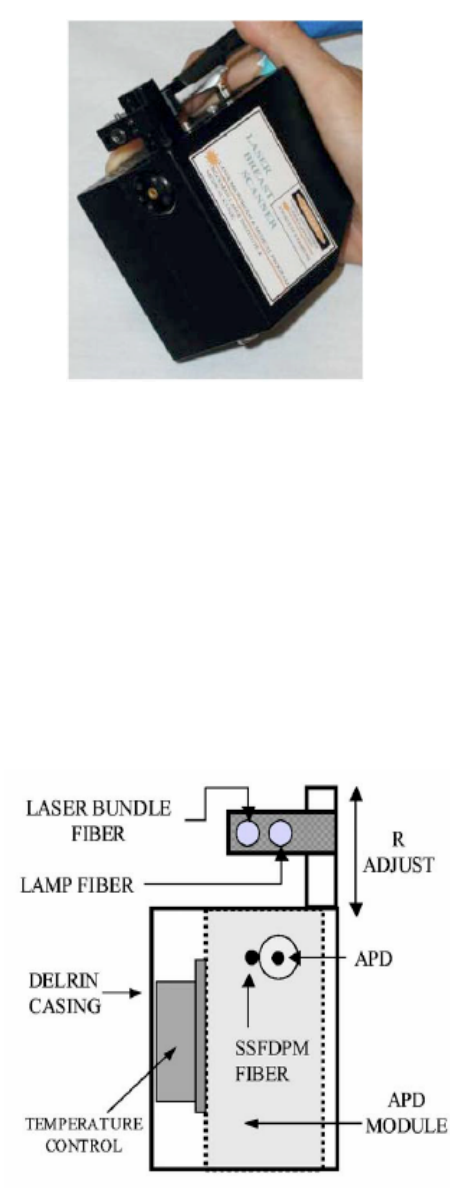


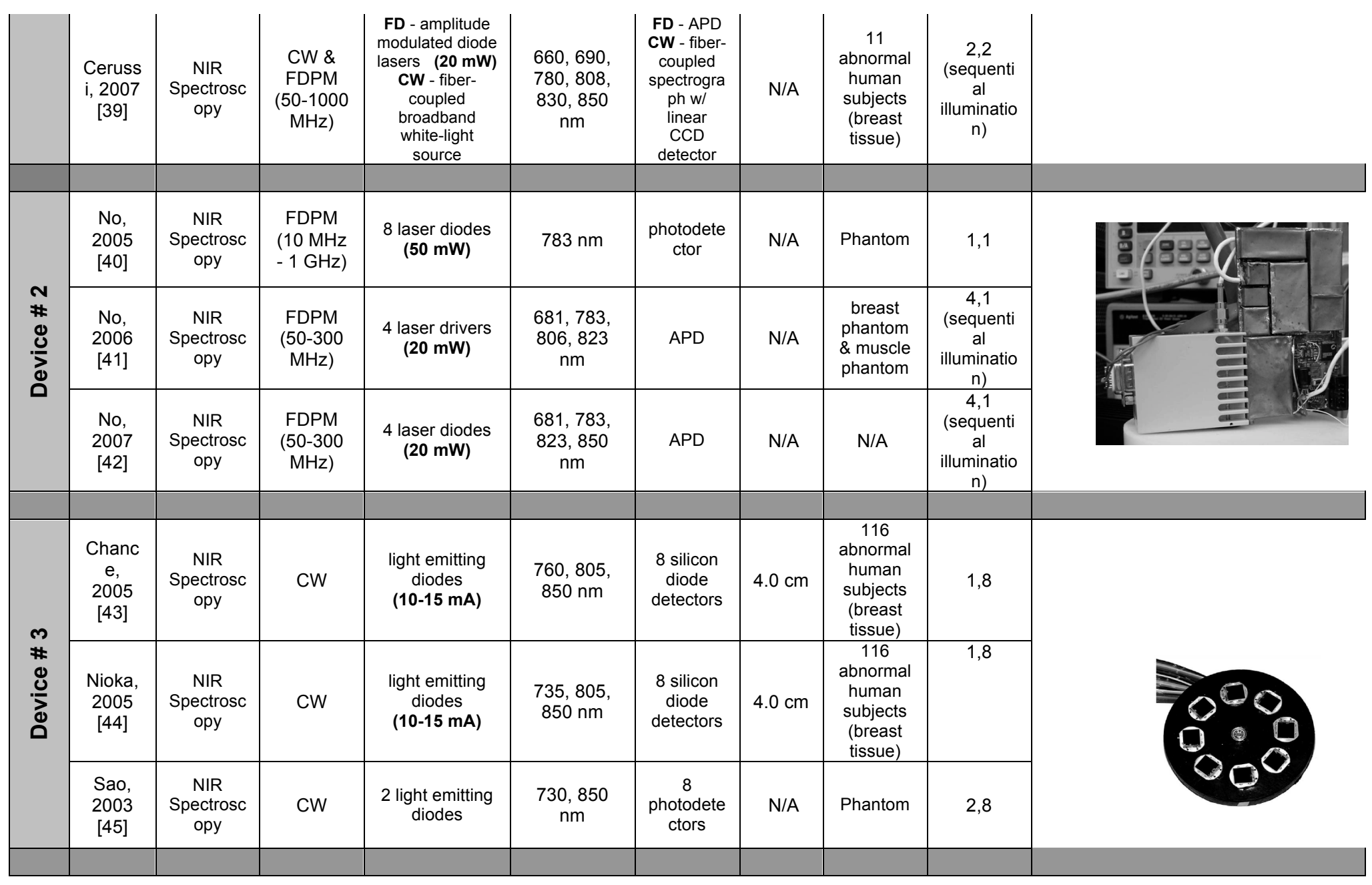




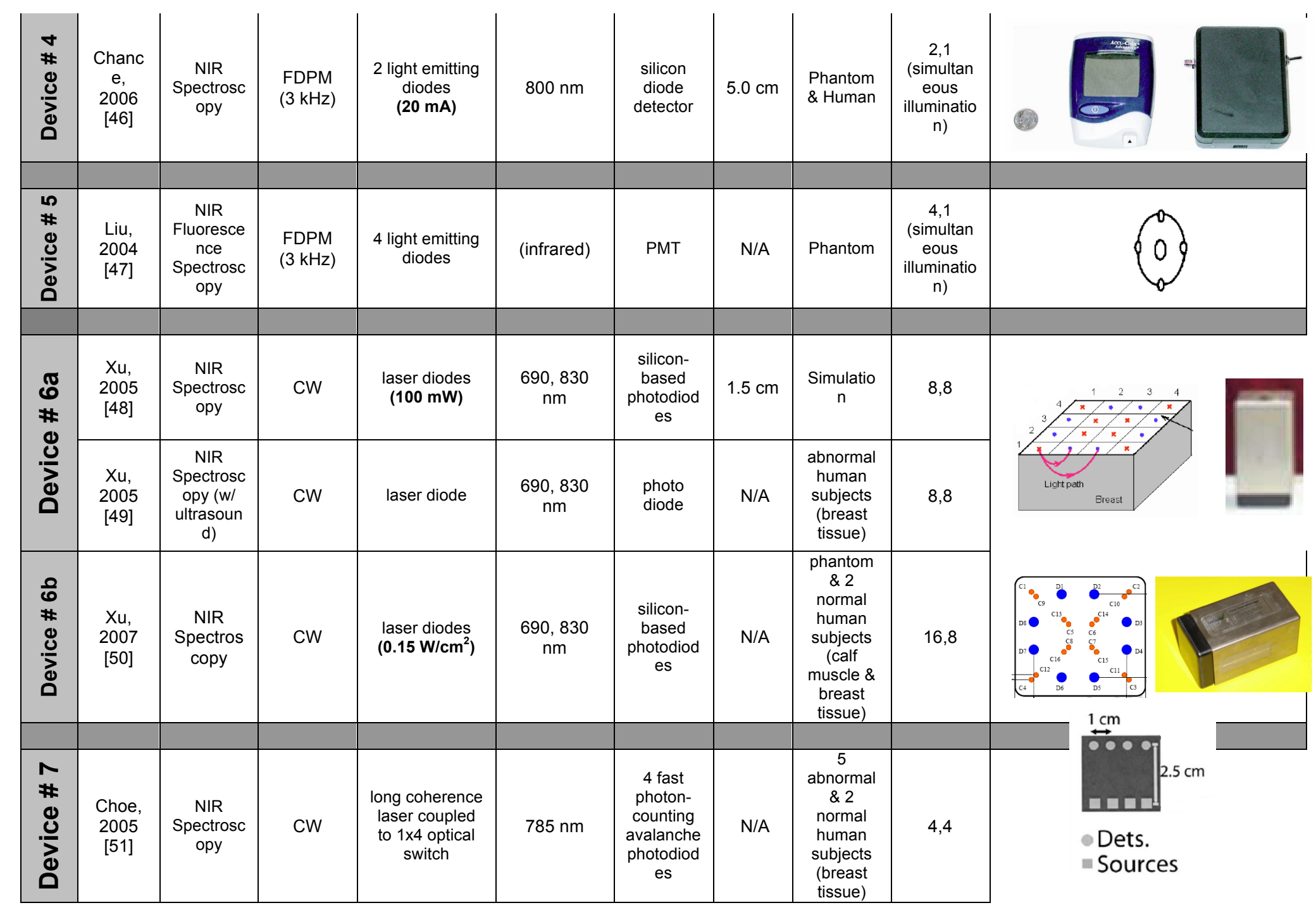




\begin{tabular}{|c|c|c|c|c|c|c|c|c|c|c|}
\hline & $\begin{array}{c}\text { Durdur } \\
\text { an, } \\
2005 \\
{[52]}\end{array}$ & $\begin{array}{c}\text { NIR } \\
\text { Spectrosc } \\
\text { opy }\end{array}$ & CW & $\begin{array}{l}\text { long coherence } \\
\text { laser coupled } \\
\text { to } 1 \times 4 \text { optical } \\
\text { switch }\end{array}$ & $785 \mathrm{~nm}$ & $\begin{array}{c}4 \text { fast } \\
\text { photon- } \\
\text { counting } \\
\text { avalanche } \\
\text { photodiod } \\
\text { es }\end{array}$ & $1.0 \mathrm{~cm}$ & Human & 4,4 & \\
\hline $\begin{array}{l}\infty \\
\# \\
0 \\
0 \\
0 \\
0\end{array}$ & $\begin{array}{l}\text { Liebert } \\
, 2005 \\
{[53]}\end{array}$ & $\begin{array}{c}\text { NIR } \\
\text { Spectrosc } \\
\text { opy }\end{array}$ & TDPM & $\begin{array}{l}2 \text { picosecond } \\
\text { diode lasers }\end{array}$ & $\begin{array}{c}803,807 \\
n m\end{array}$ & PMTs & $\mathrm{N} / \mathrm{A}$ & $\begin{array}{l}2 \text { normal } \\
\& 2 \\
\text { abnormal } \\
\text { human } \\
\text { subjects } \\
\text { (brain } \\
\text { tissue) }\end{array}$ & 2,4 & $\begin{array}{l}\text { - sources } \\
\text { - detectors }\end{array}$ \\
\hline 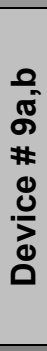 & $\begin{array}{l}\text { Sunar, } \\
2006 \\
{[54]}\end{array}$ & $\begin{array}{c}\text { Diffuse } \\
\text { Correlatio } \\
n \\
\text { Spectrosc }^{\text {opy* }} \\
\text { opy }^{*} \\
\text { (DCS) \& } \text { Diffuse } \\
\text { Reflectanc } \\
e \\
\text { Spectrosc } \\
\text { opy }^{* *} \\
\text { (DRS) } \\
\end{array}$ & $\begin{array}{l}\text { DCS - } \\
\text { CW } \\
\text { DRS - } \\
\text { FDPM } \\
(70 \mathrm{MHz})\end{array}$ & $\begin{array}{l}\text { DCS - long } \\
\text { coherence } \\
\text { laser } \\
\text { DRS - laser } \\
\text { diodes }\end{array}$ & $\begin{array}{c}\text { CW - } 785 \\
\text { nm FDPM - } \\
690,785 \\
830 \mathrm{~nm}\end{array}$ & $\begin{array}{c}\text { CW - } 4 \\
\text { fast } \\
\text { photon- } \\
\text { counting } \\
\text { avalanche } \\
\text { photodiod } \\
\text { es } \\
\text { FDPM - } 2 \\
\text { avalanche } \\
\text { photodete } \\
\text { ctors }\end{array}$ & $1.5 \mathrm{~cm}$ & $\begin{array}{c}8 \\
\text { abnormal } \\
\text { human } \\
\text { subjects } \\
\text { (head \& } \\
\text { neck } \\
\text { tissue) }\end{array}$ & $\begin{array}{c}\text { CW - 2,4 } \\
\text { FDPM - } \\
2,4\end{array}$ & 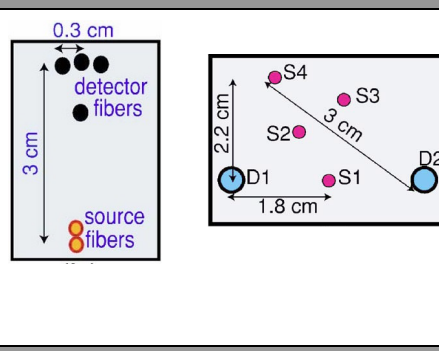 \\
\hline 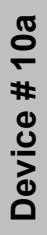 & $\begin{array}{l}\text { Zhu, } \\
1999 \\
{[55]}\end{array}$ & $\begin{array}{c}\text { NIR \& } \\
\text { Ultrasoun } \\
\quad d\end{array}$ & $\begin{array}{l}\text { FDPM } \\
(200 \\
\mathrm{MHz})\end{array}$ & $\begin{array}{l}12 \text { light } \\
\text { sources from } \\
\text { single diode } \\
\text { laser }\end{array}$ & $\begin{array}{c}776,834 \\
\mathrm{~nm}\end{array}$ & 4 APDs & $2.6 \mathrm{~cm}$ & $\begin{array}{c}\text { Breast } \\
\text { Phantom }\end{array}$ & 12,4 & $\begin{array}{c}\bullet \\
\bullet \\
\bullet \\
\bullet \\
\end{array}$ \\
\hline \multirow{3}{*}{ 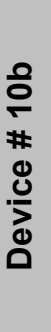 } & $\begin{array}{l}\text { Guo, } \\
2000 \\
{[56]}\end{array}$ & $\begin{array}{l}\text { NIR \& } \\
\text { Ultrasoun } \\
\quad d\end{array}$ & $\begin{array}{l}\text { FDPM } \\
(140 \\
\mathrm{MHz})\end{array}$ & $\begin{array}{l}12 \text { dual } \\
\text { wavelength } \\
\text { laser diodes }\end{array}$ & $\begin{array}{c}780,830 \\
\mathrm{~nm}\end{array}$ & 8 PMTs & $\mathrm{N} / \mathrm{A}$ & $\begin{array}{l}\text { Breast } \\
\text { Phantom }\end{array}$ & 12,8 & \multirow{3}{*}{ 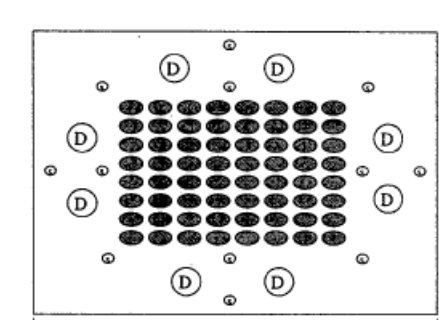 } \\
\hline & $\begin{array}{l}\text { Zhu, } \\
2000 \\
{[57]}\end{array}$ & $\begin{array}{c}\text { NIR \& } \\
\text { Ultrasoun } \\
\quad d \\
\end{array}$ & $\begin{array}{l}\text { FDPM } \\
(140 \\
\mathrm{MHz}) \\
\end{array}$ & $\begin{array}{c}12 \text { dual } \\
\text { wavelength } \\
\text { laser diodes }\end{array}$ & $\begin{array}{c}780,830 \\
\mathrm{~nm}\end{array}$ & 8 PMTs & $2.5 \mathrm{~cm}$ & $\begin{array}{l}\text { Breast } \\
\text { Phantom }\end{array}$ & 12,8 & \\
\hline & $\begin{array}{l}\text { Chen, } \\
2001 \\
{[58]}\end{array}$ & $\begin{array}{c}\text { NIR \& } \\
\text { Ultrasoun } \\
\text { d }\end{array}$ & $\begin{array}{l}\text { FDPM } \\
(140 \\
\mathrm{MHz})\end{array}$ & $\begin{array}{l}12 \text { pairs of dual } \\
\text { wavelength } \\
\text { laser diodes }\end{array}$ & $\begin{array}{c}760,830 \\
\mathrm{~nm}\end{array}$ & 8 PMTs & $2.5 \mathrm{~cm}$ & $\begin{array}{l}\text { Breast } \\
\text { Phantom }\end{array}$ & 12,8 & \\
\hline
\end{tabular}




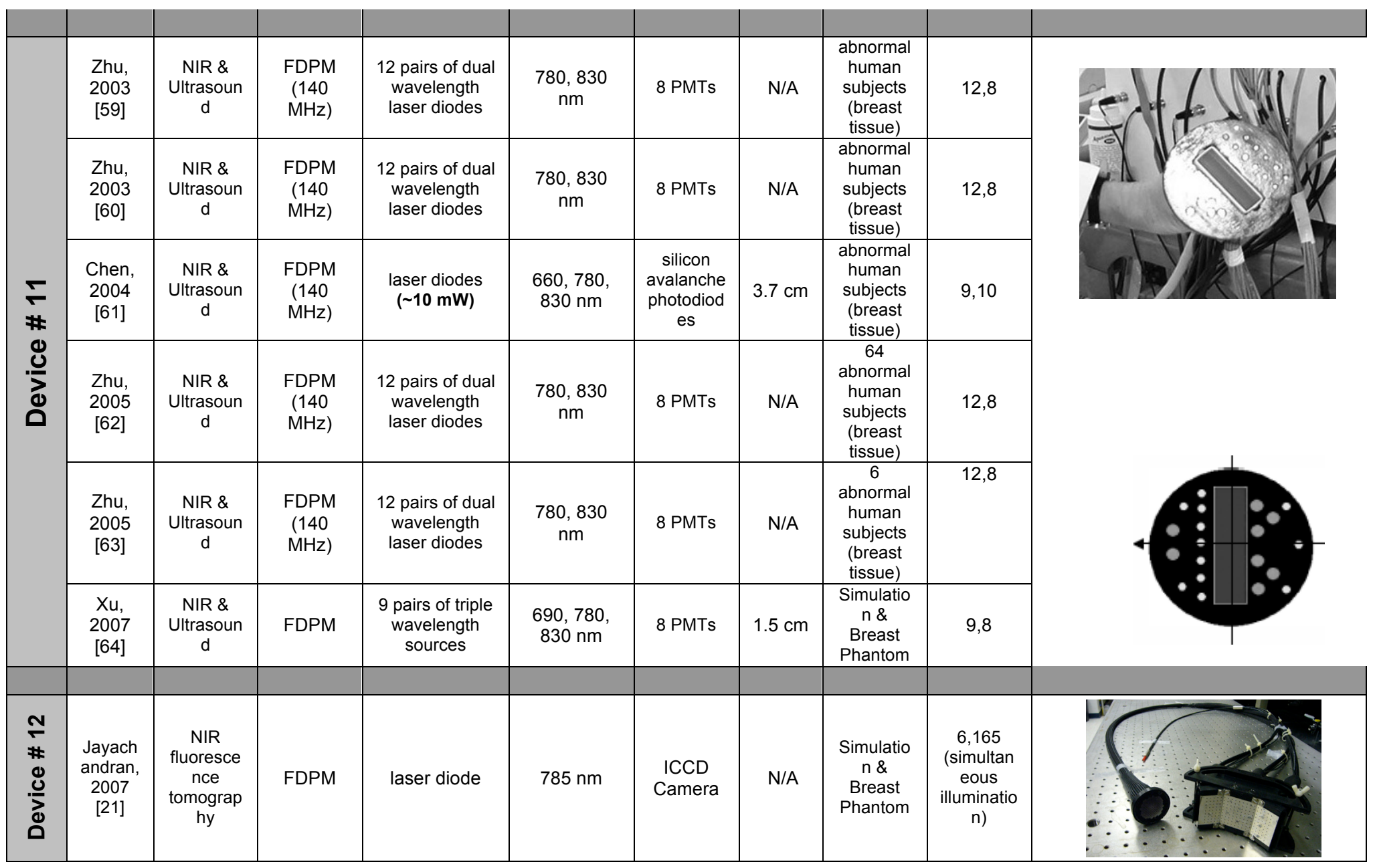


APPENDIX B Fluorescence-enhanced Diffuse Optical Tomography; Experimental Studies (Adapted from Ge, 2008 [154])

\begin{tabular}{|c|c|c|c|c|c|c|c|c|c|c|}
\hline \multirow{3}{*}{$\begin{array}{c}\text { Refer } \\
\text { ence }\end{array}$} & \multicolumn{4}{|c|}{ Phantom/Animal } & \multirow{3}{*}{$\begin{array}{c}\text { Measur } \\
\text { e } \\
\text { method }\end{array}$} & \multicolumn{2}{|c|}{ Instrument } & \multirow{3}{*}{$\begin{array}{c}2 \mathrm{D} \\
\text { or } \\
3 \mathrm{D}\end{array}$} & \multirow{3}{*}{$\begin{array}{l}\text { Forward } \\
\text { Method }\end{array}$} & \multirow{3}{*}{$\begin{array}{l}\text { Inverse } \\
\text { Method }\end{array}$} \\
\hline & \multirow{2}{*}{$\begin{array}{c}\text { Backgrou } \\
\text { nd }\end{array}$} & \multirow{2}{*}{ Target } & \multicolumn{2}{|c|}{ Contrast } & & \multirow{2}{*}{$\begin{array}{c}\text { Source } \\
\text { (geometr } \\
y)\end{array}$} & \multirow{2}{*}{$\begin{array}{c}\text { Detector } \\
\text { (geometry) }\end{array}$} & & & \\
\hline & & & Agent & Ratio & & & & & & \\
\hline $\begin{array}{c}\text { (Wu et } \\
\text { al., } \\
1995)\end{array}$ & $\begin{array}{l}\text { Cylinder } \\
\mathrm{R}=3.5 \\
\mathrm{~cm} \text { glass } \\
\text { beaker }\end{array}$ & $\begin{array}{c}\text { Sphere } \\
\mathrm{R}=1.5 \\
\mathrm{~mm}\end{array}$ & $\begin{array}{c}\text { Diethylthi } \\
\text { atricarboc } \\
\text { yanine }\end{array}$ & $1: 0$ & TDPM & $\begin{array}{c}\text { Ti: } \\
\text { Sapphire } \\
\text { laser } \\
\text { (point ) }\end{array}$ & $\begin{array}{c}\text { Streak } \\
\text { camera } \\
(\operatorname{sim} \text { point })\end{array}$ & $3 \mathrm{D}$ & None & $\begin{array}{c}\text { Localizatio } \\
\mathrm{n}\end{array}$ \\
\hline $\begin{array}{c}\text { (Wuet } \\
\text { al., } \\
1997)\end{array}$ & $\begin{array}{c}\text { Cylinder } \\
\mathrm{R}=3.2 \\
\mathrm{~cm} \text { glass } \\
\text { beaker }\end{array}$ & $\begin{array}{l}\text { Glass } \\
\text { cell }\end{array}$ & $\begin{array}{c}\text { HITCI } \\
\text { Iodide dye }\end{array}$ & $1: 0$ & TDPM & $\begin{array}{c}\text { Ti: } \\
\text { Sapphire } \\
\text { laser } \\
\text { (point) }\end{array}$ & $\begin{array}{c}\text { Streak } \\
\text { camera } \\
\text { (sim. point) }\end{array}$ & $2 \mathrm{D}$ & Analytical & $\begin{array}{l}\text { Laplace } \\
\text { transform }\end{array}$ \\
\hline $\begin{array}{c}\text { (Chan } \\
\text { g et } \\
\text { al. } \\
\text { 1997) }\end{array}$ & $\begin{array}{c}\text { Cylinder } \\
\mathrm{R}=4 \mathrm{~cm} \\
\mathrm{H}=50 \mathrm{~cm}\end{array}$ & $\begin{array}{c}\text { Balloon } \\
\mathrm{V}= \\
0.5 \mathrm{~mL}\end{array}$ & $\begin{array}{c}\text { Rhodamin } \\
\text { e } 6 \mathrm{G} \text { dye }\end{array}$ & $500: 1$ & CW & $\begin{array}{l}\text { Argon ion } \\
\text { laser } \\
\text { (area) }\end{array}$ & $\begin{array}{c}\text { CCD camera } \\
\text { (area) }\end{array}$ & $2 \mathrm{D}$ & NS & $\begin{array}{l}\text { POCS, } \\
\text { CGD, } \\
\text { SART }\end{array}$ \\
\hline $\begin{array}{l}\text { (Hull } \\
\text { et al., } \\
1998)\end{array}$ & $\begin{array}{c}\text { Slab } \\
14.5 \mathrm{~cm} \\
\times 29.5 \mathrm{~cm} \\
\times 15.5 \mathrm{~cm}\end{array}$ & $\begin{array}{c}\text { Spheric } \\
\text { al glass } \\
\text { bulb } \\
\mathrm{R}=3.0 \\
\mathrm{~mm}\end{array}$ & $\begin{array}{c}\text { Nile blue } \\
\text { A }\end{array}$ & $1: 0$ & $\mathrm{CW}$ & $\begin{array}{l}\text { Dye laser } \\
\text { (area) }\end{array}$ & $\begin{array}{c}\text { CCD camera } \\
\text { (sim. point) }\end{array}$ & $2 \mathrm{D}$ & Monte carlo & $\begin{array}{c}\text { Localizatio } \\
n\end{array}$ \\
\hline $\begin{array}{c}\text { (Chern } \\
\text { omord } \\
\text { ik et } \\
\text { al., } \\
1999)\end{array}$ & $\begin{array}{l}\text { Delrin } \\
\text { phantom } \\
\text { NS for } \\
\text { size }\end{array}$ & $\begin{array}{l}\mathrm{V}=1 \\
\mathrm{~mm}^{3}\end{array}$ & $\begin{array}{c}\text { Rhodamin } \\
\text { e }\end{array}$ & $1: 0$ & $\mathrm{CW}$ & $\begin{array}{l}\text { Argon } \\
\text { laser } \\
\text { (area) }\end{array}$ & $\begin{array}{c}\text { CCD camera } \\
\text { (area) }\end{array}$ & $\begin{array}{l}2 \mathrm{D} / \\
3 \mathrm{D}\end{array}$ & NS & $\begin{array}{l}\text { Random } \\
\text { walk } \\
\text { theory }\end{array}$ \\
\hline $\begin{array}{c}\text { (Hawr } \\
\text { ysz et } \\
\text { al., } \\
2001 \text {; } \\
\text { Eppste } \\
\text { in } \text { et } \\
\text { al., }\end{array}$ & $\begin{array}{c}\text { Slab } \\
\mathrm{S}=8^{2} \mathrm{~cm}^{2} \\
\mathrm{H}=4 \mathrm{~cm}\end{array}$ & $\begin{array}{l}\text { Cube } \\
1 \mathrm{~cm}^{3}\end{array}$ & ICG & $\begin{array}{c}1: 0 \& \\
\text { imper } \\
\text { fect } \\
\text { uptak } \\
\text { e }\end{array}$ & FDPM & $\begin{array}{l}\text { Laser } \\
\text { diode } \\
\text { (seq. } \\
\text { point) }\end{array}$ & $\begin{array}{c}\text { PMT } \\
\text { (seq. point) }\end{array}$ & $3 \mathrm{D}$ & MFD & AEKF \\
\hline
\end{tabular}




\begin{tabular}{|c|c|c|c|c|c|c|c|c|c|c|}
\hline 2002) & & & & & & & & & & \\
\hline $\begin{array}{l}\text { (Ntzia } \\
\text { christo } \\
\text { s and } \\
\text { Weissl } \\
\text { eder, } \\
2001)\end{array}$ & $\begin{array}{c}\text { Cylinder } \\
\mathrm{R}=1.25 \\
\mathrm{~cm} \\
\mathrm{H}=15 \mathrm{~cm}\end{array}$ & $\begin{array}{c}\text { Cylinde } \\
\mathrm{r} \\
\mathrm{R}= \\
1.75 \mathrm{~mm} \\
\mathrm{H}= \\
1,: 3.5 \\
\mathrm{~cm}\end{array}$ & $\begin{array}{c}\text { ICG in } \\
\text { backgroun } \\
\mathrm{d} \text { and } \\
\text { Cy5.5 as } \\
\text { contrast } \\
\text { tumor }\end{array}$ & $1: 0$ & $\mathrm{CW}$ & $\begin{array}{c}\text { Laser } \\
\text { diode } \\
\text { (seq.point) }\end{array}$ & $\begin{array}{c}\text { CCD camera } \\
\text { (sim. Point) }\end{array}$ & $3 \mathrm{D}$ & FD & SIRT \\
\hline $\begin{array}{c}\text { (Ntzia } \\
\text { christo } \\
\text { s et } \\
\text { al., } \\
2002) \\
\end{array}$ & $\begin{array}{l}\text { In vivo } \\
\text { (rat) }\end{array}$ & $\begin{array}{l}\text { Tumor } \\
\text { NS for } \\
\text { size }\end{array}$ & $\begin{array}{c}\text { Enzyme } \\
\text { activatabl } \\
\mathrm{e} \\
\text { fluorochro } \\
\text { mes }\end{array}$ & $1: 0$ & CW & $\begin{array}{c}\text { Laser } \\
\text { diode } \\
\text { (seq. } \\
\text { point/ } \\
\text { area) }\end{array}$ & $\begin{array}{c}\text { CCD camera } \\
\text { (area) }\end{array}$ & $3 \mathrm{D}$ & FD & $\begin{array}{c}\text { Constraine } \\
\text { d ART }\end{array}$ \\
\hline $\begin{array}{c}\text { (Lee } \\
\text { and } \\
\text { Sevick } \\
- \\
\text { Murac } \\
\text { a, } \\
2001 ; \\
2002) \\
\end{array}$ & $\begin{array}{c}\text { Slab } \\
\mathrm{V}= \\
8 \times 8 \times 8 \\
\mathrm{~cm}^{3} \\
\mathrm{~V}= \\
8 \times 4 \times 8 \\
\mathrm{~cm}^{3}\end{array}$ & $\begin{array}{c}\text { Cube } \\
\mathrm{V}= \\
1 \mathrm{~cm}^{3}\end{array}$ & ICG & 100:1 & FDPM & $\begin{array}{c}\text { Laser } \\
\text { diode } \\
\text { (seq. } \\
\text { point) }\end{array}$ & $\begin{array}{c}\text { PMT } \\
\text { (seq.point) }\end{array}$ & $3 \mathrm{D}$ & MFD & $\begin{array}{l}\text { Leverberg- } \\
\text { Marquardt }\end{array}$ \\
\hline $\begin{array}{c}\text { (Grave } \\
\text { s et } \\
\text { al., } \\
2003 \text { ) }\end{array}$ & $\begin{array}{l}\text { In vivo } \\
\text { (rat) }\end{array}$ & $\begin{array}{c}\text { Tumor } \\
\mathrm{R}=1.5 \\
\mathrm{~mm}\end{array}$ & $\begin{array}{c}\text { Cy5.5 } \\
\text { based } \\
\text { activatabl } \\
\text { e } \\
\text { fluorescen } \\
\text { t probe }\end{array}$ & $1: 0$ & CW & $\begin{array}{c}\text { Laser } \\
\text { diode } \\
\text { (seq. } \\
\text { point/area } \\
\text { ) }\end{array}$ & $\begin{array}{c}\text { CCD camera } \\
\text { (area) }\end{array}$ & $3 \mathrm{D}$ & FD & SVD \\
\hline $\begin{array}{c}\text { (Goda } \\
\text { varty } \\
\text { et al., } \\
2003 ; \\
\text { Godav } \\
\text { arty et } \\
\text { al., } \\
2004 \mathrm{~b} ; \\
\text { Godav } \\
\text { arty et } \\
\text { al., }\end{array}$ & $\begin{array}{c}\text { Breast } \\
\text { phantom } \\
\mathrm{V}=1087 \\
\mathrm{~cm}^{3} \\
\& \text { Semi- } \\
\text { infinite } \\
\text { phantom }\end{array}$ & $\begin{array}{c}\text { Target } \\
\mathrm{V}= \\
1 \mathrm{~cm}^{3}\end{array}$ & ICG & $\begin{array}{c}100: 1 \\
1: 0\end{array}$ & FDPM & $\begin{array}{c}\text { Modulate } \\
\text { d laser } \\
\text { diode } \\
\text { (seq./sim. } \\
\text { Point) }\end{array}$ & $\begin{array}{c}\text { CCD camera } \\
\text { (sim.point) }\end{array}$ & $3 \mathrm{D}$ & FEM & AEKF \\
\hline
\end{tabular}




\begin{tabular}{|c|c|c|c|c|c|c|c|c|c|c|}
\hline 2004a) & & & & & & & & & & \\
\hline $\begin{array}{l}\text { (Klose } \\
\text { et al., } \\
2005)\end{array}$ & $\begin{array}{c}\mathrm{Slab} \\
\mathrm{S}=4^{2} \\
\mathrm{~cm}^{2} \\
\mathrm{H}=1.3 \\
\mathrm{~cm}\end{array}$ & $\begin{array}{c}\text { Tube } \\
\mathrm{R}= \\
0.1 \mathrm{~cm} \\
\mathrm{H}=4 \\
\mathrm{~cm}\end{array}$ & Сy5.5 & $1: 0$ & $\mathrm{CW}$ & $\begin{array}{c}\text { Laser } \\
\text { diode } \\
\text { (seq. } \\
\text { point/ } \\
\text { area) }\end{array}$ & $\begin{array}{c}\text { CCD camera } \\
\text { (area) }\end{array}$ & $3 \mathrm{D}$ & $\begin{array}{c}\text { FD based on } \\
\text { ERT }\end{array}$ & $\begin{array}{c}\text { Nonlinear } \\
\text { optimizatio } \\
\mathrm{n}\end{array}$ \\
\hline $\begin{array}{c}\text { (Wu et } \\
\text { al., } \\
2005 \mathrm{~b} ; \\
\text { Wu et } \\
\text { al., } \\
2005 \mathrm{a})\end{array}$ & $\begin{array}{c}\text { Cylinder } \\
\mathrm{R}=25 \mathrm{~mm} \\
\mathrm{H}= \\
100 \mathrm{~mm}\end{array}$ & $\begin{array}{c}\text { Tube } \\
\mathrm{R}=7 \\
\mathrm{~mm}\end{array}$ & $\begin{array}{c}\text { Metal } \\
\text { nanoshell; } \\
\text { Hybrid } \\
\text { virus-dye } \\
\text { nanopartic } \\
\text { le }\end{array}$ & $1: 0$ & CW & $\begin{array}{c}\text { Laser } \\
\text { diode } \\
\text { (seq.point) }\end{array}$ & $\begin{array}{c}\text { PMT } \\
\text { (seq. point) }\end{array}$ & $2 \mathrm{D}$ & FEM & $\begin{array}{c}\text { Newton's } \\
\text { iterative } \\
\text { method }\end{array}$ \\
\hline $\begin{array}{l}\text { (Roy, } \\
2005)\end{array}$ & $\begin{array}{c}\text { Cube } \\
V=8^{3} \mathrm{~cm}^{3}\end{array}$ & $\begin{array}{c}\text { Cube } \\
\mathrm{V}=1 \mathrm{~cm}^{3}\end{array}$ & ICG & $\begin{array}{l}\text { Perfe } \\
\text { ct and } \\
100: 1\end{array}$ & FDPM & $\begin{array}{l}\text { Laser } \\
\text { diode } \\
\text { (area) }\end{array}$ & $\begin{array}{c}\text { CCD camera } \\
\text { (area) }\end{array}$ & $3 \mathrm{D}$ & $\begin{array}{c}\text { Experimenta } \\
1 \text { method }\end{array}$ & $\begin{array}{c}\mathrm{PMBF} / \mathrm{CO} \\
\mathrm{NTN}\end{array}$ \\
\hline $\begin{array}{l}\text { (Goda } \\
\text { varty } \\
\text { et al., } \\
2005)\end{array}$ & $\begin{array}{c}\text { Breast } \\
\text { phantom } \\
\mathrm{V}= \\
1087 \mathrm{~cm}^{3}\end{array}$ & $\begin{array}{l}\mathrm{V}=1 \\
\mathrm{~cm}^{3}\end{array}$ & $\begin{array}{c}\text { ICG; } \\
\text { 3-3'- } \\
\text { Diethylthi } \\
\text { atricarboc } \\
\text { yanine } \\
\text { iodide }\end{array}$ & $\begin{array}{c}70: 1 \\
100: 1 \\
\text { Lifeti } \\
\text { me } \\
1: 1 \\
2.1: 1\end{array}$ & FDPM & $\begin{array}{l}\text { Laser } \\
\text { diode } \\
\text { (area) }\end{array}$ & $\begin{array}{c}\text { CCD camera } \\
\text { (area) }\end{array}$ & $3 \mathrm{D}$ & FEM & AEKF \\
\hline $\begin{array}{l}\text { (Chen } \\
\text { et al., } \\
2005)\end{array}$ & $\begin{array}{c}\text { Slab } \\
\text { phantom } \\
\mathrm{V}=12 \times \\
5 \times 12 \\
\mathrm{~cm}^{3} \\
\text { In vivo }\end{array}$ & $\begin{array}{l}\text { Cube } \\
\mathrm{V}= \\
1 \mathrm{~cm}^{3}\end{array}$ & $\begin{array}{c}\text { NIR804- } \\
\text { 2-D } \\
\text { Glucosam } \\
\text { ide }\end{array}$ & $1: 0$ & FDPM & $\begin{array}{l}\text { Laser } \\
\text { diode } \\
\text { (sim. } \\
\text { Point) }\end{array}$ & $\begin{array}{c}\text { PMT } \\
\text { (seq. point) }\end{array}$ & $2 \mathrm{D}$ & Analytical & $\begin{array}{c}\text { Back- } \\
\text { projection }\end{array}$ \\
\hline $\begin{array}{l}\text { (Patwa } \\
\text { rdhan } \\
\text { et al., } \\
\text { 2005) }\end{array}$ & $\begin{array}{l}\text { In vivo } \\
\text { (mice) }\end{array}$ & $\begin{array}{l}\text { Tumor } \\
\text { up to } 5 \\
\text { mm }\end{array}$ & ICG & N/A & $\mathrm{CW}$ & $\begin{array}{l}\text { Laser } \\
\text { diode } \\
\text { (seq. } \\
\text { point0 }\end{array}$ & $\begin{array}{c}\text { CCD camera } \\
\text { (area) }\end{array}$ & $3 \mathrm{D}$ & NS & ART \\
\hline $\begin{array}{l}\text { (Zacha } \\
\text { rakis } \\
\text { et al., } \\
2005)\end{array}$ & $\begin{array}{l}\text { In vivo } \\
\text { (mice) }\end{array}$ & $\begin{array}{l}\text { A thin } \\
\text { glass } \\
\text { capillar } \\
\text { y tube }\end{array}$ & $\begin{array}{c}\text { fluorescei } \\
n \\
\text { isothiocya } \\
\text { nate }\end{array}$ & $1: 0$ & CW & $\begin{array}{l}\text { Laser } \\
\text { diode } \\
\text { (seq. } \\
\text { point/ }\end{array}$ & $\begin{array}{c}\text { CCD camera } \\
\text { (area) }\end{array}$ & $3 \mathrm{D}$ & FD & ART \\
\hline
\end{tabular}




\begin{tabular}{|c|c|c|c|c|c|c|c|c|c|c|}
\hline & & & & & & area) & & & & \\
\hline $\begin{array}{l}\text { (Fedel } \\
\text { e et } \\
\text { al., } \\
2005)\end{array}$ & $\begin{array}{l}\text { Breast } \\
\text { phantom } \\
1087 \mathrm{~cm}^{3}\end{array}$ & $\begin{array}{l}\text { Cube } \\
1 \mathrm{cc}\end{array}$ & ICG & $100: 1$ & FDPM & $\begin{array}{l}\text { Modulate } \\
\text { d laser } \\
\text { diode } \\
\text { (seq. } \\
\text { point) }\end{array}$ & $\begin{array}{c}\text { CCD camera } \\
\text { (sim. Point) }\end{array}$ & $3 \mathrm{D}$ & BEM & AEKF \\
\hline $\begin{array}{l}\text { (Joshi } \\
\text { et al, } \\
2006)\end{array}$ & $\begin{array}{c}\text { Cube } \\
\text { phantom } \\
\mathrm{V}=8 \mathrm{x} 8 \mathrm{x} 8 \mathrm{c} \\
\mathrm{m}^{3}\end{array}$ & $\begin{array}{c}\text { Bulb } \\
\text { (dia: 3- } \\
\text { 4mm) } \\
\text { Depth: } \\
\text { 1-2cm } \\
\text { (edge) }\end{array}$ & ICG & $1: 0$ & FDPM & $\begin{array}{l}\text { Laser } \\
\text { diode } \\
\text { (area ) }\end{array}$ & $\begin{array}{c}\text { CCD } \\
\text { camera } \\
\text { (area) }\end{array}$ & $3 \mathrm{D}$ & FEM & AEKF \\
\hline $\begin{array}{l}\text { (Roy } \\
\text { et al, } \\
2006 \text { ) }\end{array}$ & $\begin{array}{c}\text { Breast } \\
\text { phantom } \\
\mathrm{V}= \\
1087 \mathrm{~cm}^{3}\end{array}$ & $\begin{array}{c}\text { Bulb } \\
(0.5- \\
\left.1.0 \mathrm{~cm}^{3}\right)\end{array}$ & ICG & $\begin{array}{c}1: 0 \\
100: 1\end{array}$ & FDPM & $\begin{array}{c}\text { Laser } \\
\text { diode } \\
\text { (seq.point) }\end{array}$ & $\begin{array}{l}\text { CCD camera } \\
\text { (sim.point) }\end{array}$ & $3 \mathrm{D}$ & FEM & $\begin{array}{c}\mathrm{PMBF} / \mathrm{CO} \\
\mathrm{NTN}\end{array}$ \\
\hline $\begin{array}{l}\text { (Schul } \\
\mathrm{z} \text { et al, } \\
2006)\end{array}$ & $\begin{array}{l}\text { cylindrical } \\
\text { phantom [ } \\
\mathrm{D}=4 \mathrm{~cm}, \\
\text { height } 10 \\
\mathrm{~cm} ;\end{array}$ & $\begin{array}{c}\text { hollow } \\
\text { tubes( } \\
\mathrm{D}=4 \\
\mathrm{~mm})\end{array}$ & Су5.5 & 1: 0 & $\mathrm{CW}$ & $\begin{array}{c}\text { Laser } \\
\text { diode } \\
\text { (seq.point } \\
\& \\
\text { Area) }\end{array}$ & $\begin{array}{c}\text { CCD camera } \\
\text { (sim. point } \\
\&\end{array}$ & $3 \mathrm{D}$ & FEM & $\begin{array}{c}\text { Normalized } \\
\text { Born } \\
\text { approach }\end{array}$ \\
\hline $\begin{array}{l}\text { (Yuan } \\
\text { and } \\
\text { Zhu et } \\
\text { al } \\
\text { 2006) }\end{array}$ & $\begin{array}{l}\text { Semi- } \\
\text { infinite } \\
\text { phantom }\end{array}$ & $\begin{array}{c}\text { Spheric } \\
\mathrm{al}(\mathrm{R}=0 . \\
4 \mathrm{~cm}) \\
\text { Cube } \\
(0.8 \times 0.8 \\
\times 0.8 \mathrm{~cm}^{3} \\
)\end{array}$ & Су5.5 & $\begin{array}{c}1: 0 \\
100: 1 \\
200: 1\end{array}$ & FDPM & $\begin{array}{c}\text { Laser } \\
\text { diode } \\
\text { (seq.point) }\end{array}$ & $\begin{array}{c}\text { PMT(seq.po } \\
\text { int) }\end{array}$ & $3 \mathrm{D}$ & $\begin{array}{l}\text { Normalized } \\
\text { Born } \\
\text { Approximati } \\
\text { on } \\
\text { (analytical) }\end{array}$ & $\begin{array}{l}\text { Total least } \\
\text { square } \\
\text { /Conjugate } \\
\text { gradient } \\
\text { technique }\end{array}$ \\
\hline $\begin{array}{l}\text { (Moha } \\
\text { jerani } \\
\text { et al, } \\
2007)\end{array}$ & $\begin{array}{c}\text { Cylinder } \\
(\mathrm{D}=7.5 \\
\mathrm{cm}, \mathrm{H}=6 \\
\mathrm{~cm})\end{array}$ & $\begin{array}{c}\text { Small } \\
\text { glass } \\
\text { cuvetts } \\
(1 \mathrm{~mm} \\
\times 1 \mathrm{~mm} \\
\times 3 \\
\mathrm{~mm}) \\
\end{array}$ & $\begin{array}{l}\text { Rhodamin } \\
\text { e } 6 \mathrm{G}\end{array}$ & $1: 0$ & $\mathrm{CW}$ & $\begin{array}{l}\text { Solid-state } \\
\text { laser (seq. } \\
\text { point) }\end{array}$ & $\begin{array}{l}\text { PMT (seq. } \\
\text { Point) }\end{array}$ & $3 \mathrm{D}$ & FEM & $\begin{array}{l}\text { Sparse } \\
\text { model }\end{array}$ \\
\hline $\begin{array}{l}\text { Corlu } \\
\text { et al, } \\
2007) \\
\end{array}$ & $\begin{array}{l}\text { In vivo } \\
\text { (human) }\end{array}$ & Tumor & ICG & $\begin{array}{c}3.5- \\
5.5: 1\end{array}$ & $\mathrm{CW}$ & $\begin{array}{l}\text { Laser } \\
\text { diode } \\
\text { (seq. }\end{array}$ & $\begin{array}{c}\text { CCD camera } \\
\text { (Area) }\end{array}$ & $3 \mathrm{D}$ & FEM & GMRES \\
\hline
\end{tabular}




\begin{tabular}{|c|c|c|c|c|c|c|c|c|c|c|}
\hline & & & & & & point) & & & & \\
\hline $\begin{array}{l}\text { (Davis } \\
\text { et al, } \\
2007 \text { ) }\end{array}$ & $\begin{array}{c}\text { Rounded } \\
\text { epoxy } \\
\text { solid } \\
\text { phantom } \\
(\mathrm{D}=5.5 \mathrm{~cm} \\
)\end{array}$ & $\begin{array}{c}14 \mathrm{~mm} \\
\text { hole }\end{array}$ & Lutex & $\begin{array}{l}2.0: 1 \\
3.3: 1\end{array}$ & $\begin{array}{c}\mathrm{CW} / \mathrm{FD} \\
\mathrm{PM}\end{array}$ & $\begin{array}{c}\text { Laser } \\
\text { diode } \\
\text { (seq.point) }\end{array}$ & $\begin{array}{c}\text { CCD camera } \\
\text { sim.point) }\end{array}$ & $3 \mathrm{D}$ & FEM & $\begin{array}{l}\text { Adjoint } \\
\text { Method } \\
\text { (MR- } \\
\text { guided) }\end{array}$ \\
\hline $\begin{array}{l}\text { (Delio } \\
\text { lanis } \\
\text { et al, } \\
\text { 2007) }\end{array}$ & $\begin{array}{l}\text { in vivo } \\
\text { (mice) }\end{array}$ & $\begin{array}{c}9 \mathrm{~mm} \\
\text { tube } \\
(0.75 \mathrm{~m} \\
\mathrm{m} \\
\text { diamete } \\
\mathrm{r}) \\
\end{array}$ & $\begin{array}{c}\text { Alexa } \\
\text { Fluor } 750\end{array}$ & $1: 0$ & $\mathrm{CW}$ & $\begin{array}{l}\text { Laser } \\
\text { diode } \\
\text { (seq. } \\
\text { point) }\end{array}$ & $\begin{array}{c}\text { CCD camera } \\
\text { (area) }\end{array}$ & $3 \mathrm{D}$ & Analytical & $\begin{array}{c}\text { Analytical } \\
\text { (Normalize } \\
\text { d Born } \\
\text { solution) }\end{array}$ \\
\hline $\begin{array}{l}\text { (Herve } \\
\text { et al, } \\
2007 \text { ) }\end{array}$ & $\begin{array}{c}\text { Solid } \\
\text { phantom } \\
\text { And in } \\
\text { vivo } \\
\text { (mice) }\end{array}$ & $\begin{array}{c}\text { Glass } \\
\text { tube } \\
(\mathrm{D}=3 \\
\mathrm{mm}, \mathrm{L}= \\
3 \mathrm{~cm}) \\
\text { and } 14- \\
\text { day } \\
\text { lung } \\
\text { tumor in } \\
\text { mice }\end{array}$ & Alexa 750 & $1: 0$ & $\mathrm{CW}$ & $\begin{array}{l}\text { Laser } \\
\text { diode } \\
\text { (seq. } \\
\text { point) }\end{array}$ & $\begin{array}{c}\text { CCD camera } \\
\text { (area) }\end{array}$ & $3 \mathrm{D}$ & FD & ART \\
\hline $\begin{array}{c}\text { (Keps } \\
\text { hire et } \\
\text { al, } \\
2007 ; \\
2008)\end{array}$ & $\begin{array}{c}\text { Tank } \\
\text { filled with } \\
2 \% \text { India } \\
\text { ink and } \\
5 \% \\
\text { Tween-20 } \\
(\mathrm{V}= \\
3 \times 4 \times 1 \mathrm{~cm} \\
3) \\
\end{array}$ & $\begin{array}{c}\text { Cyclind } \\
\text { rical } \\
\text { target } \\
(D=8 \mathrm{~m} \\
\mathrm{m})\end{array}$ & Pp-IX & $\begin{array}{c}3.5: 1 \\
, 5: 1 \\
\text { and } \\
10: 1\end{array}$ & $\mathrm{CW}$ & $\begin{array}{l}\text { Laser } \\
\text { diode } \\
\text { (seq. } \\
\text { point) }\end{array}$ & $\begin{array}{c}\text { CCD camera } \\
\text { (area) }\end{array}$ & $3 \mathrm{D}$ & FEM & $\begin{array}{l}\text { Non-liniear } \\
\text { Newton } \\
\text { minimizati } \\
\text { on }\end{array}$ \\
\hline $\begin{array}{l}\text { (Mont } \\
\text { et et } \\
\text { al, } \\
2007 \text { ) }\end{array}$ & $\begin{array}{l}\text { In vivo } \\
\text { (mice) }\end{array}$ & $\begin{array}{l}5-7 \text { day } \\
\text { tumor } \\
(\mathrm{D}=3- \\
5 \mathrm{~mm})\end{array}$ & $\begin{array}{c}\text { Angiosens } \\
\text { e } 680 \text { and } \\
750\end{array}$ & / & $\mathrm{CW}$ & $\begin{array}{l}\text { FMT- } \\
\text { Solaris }\end{array}$ & FMT-Solaris & $3 \mathrm{D}$ & $\begin{array}{l}\text { Normalized } \\
\text { Born }\end{array}$ & N/A \\
\hline
\end{tabular}




\begin{tabular}{|c|c|c|c|c|c|c|c|c|c|c|}
\hline $\begin{array}{l}\text { (Meye } \\
\text { r et al, } \\
2007 .)\end{array}$ & $\begin{array}{c}\text { In vivo } \\
\text { (transgeni } \\
\text { c mice) }\end{array}$ & tumor & CD2-GFP & I & CW & $\begin{array}{l}\mathrm{Ar}^{+} \text {laser } \\
\quad \text { (area) }\end{array}$ & $\begin{array}{c}\text { CCD camera } \\
\text { (area) }\end{array}$ & $3 \mathrm{D}$ & FD & ART \\
\hline $\begin{array}{l}\text { (Roy } \\
\text { et al, } \\
2007 \text { ) }\end{array}$ & $\begin{array}{c}\begin{array}{c}\text { Breast } \\
\text { shape }\end{array} \\
\text { phantom } \\
(\mathrm{V}=1081 \\
\left.\mathrm{cm}^{3}\right)\end{array}$ & $\begin{array}{c}\text { Spheric } \\
\text { al target } \\
(\mathrm{V}=1.0 \\
\left.\mathrm{cm}^{3}\right)\end{array}$ & ICG & $\begin{array}{c}\text { Fluor } \\
\text { escen } \\
\text { ce } \\
\text { absor } \\
\text { ption: } \\
212 \text { : } \\
1 \\
70: 1 \\
\text { Lifeti } \\
\text { me: } \\
1: 2.1 \\
2.1: \\
1\end{array}$ & FDPM & $\begin{array}{l}\text { Laser } \\
\text { diode } \\
\text { (seq. } \\
\text { point) }\end{array}$ & $\begin{array}{c}\text { CCD camera } \\
\text { (sim. point) }\end{array}$ & $3 \mathrm{D}$ & FEM & $\begin{array}{c}\mathrm{PMBF} / \mathrm{CO} \\
\text { NTN }\end{array}$ \\
\hline $\begin{array}{c}\text { (Ge et } \\
\text { al, } \\
2008)\end{array}$ & $\begin{array}{c}\text { Cube } \\
\text { filled with } \\
1 \% \\
\text { Liposyn } \\
\text { solution } \\
(\mathrm{V}=10 \\
\times 10 \times 6.5 \\
\left.\mathrm{~cm}^{3}\right)\end{array}$ & $\begin{array}{c}\text { Sphere } \\
(D=0.95 \\
\mathrm{cm})\end{array}$ & ICG & $1: 0$ & FDPM & $\begin{array}{l}\text { Laser } \\
\text { diode } \\
\text { (sim. } \\
\text { point) }\end{array}$ & $\begin{array}{c}\text { CCD camera } \\
\text { (sim. point) }\end{array}$ & $3 \mathrm{D}$ & FEM & AEKF \\
\hline $\begin{array}{c}\text { (Keren } \\
\text { et al, } \\
2008)\end{array}$ & $\begin{array}{l}\text { Cylindrica } \\
1 \text { Phantom }\end{array}$ & $\begin{array}{c}\text { Inclusio } \\
\mathrm{n}(\mathrm{D}=3 \\
\mathrm{mm})\end{array}$ & Су 5.5 & 1:0 & TD & $\begin{array}{c}\text { Pulsed } \\
\text { diode } \\
\text { laser (seq. } \\
\text { point) }\end{array}$ & $\begin{array}{l}\text { PMT (seq. } \\
\text { point) }\end{array}$ & $3 \mathrm{D}$ & $\begin{array}{c}\text { Born } \\
\text { approximati } \\
\text { on }\end{array}$ & N/A \\
\hline $\begin{array}{l}\text { (Tan } \\
\text { et al, } \\
2008)\end{array}$ & $\begin{array}{c}\text { Solid } \\
\text { phantom } \\
(\mathrm{V}=3 \times 3 \\
\left.\times 9 \mathrm{~cm}^{3}\right)\end{array}$ & $\begin{array}{c}\text { Cylindri } \\
\text { cal } \\
\text { target } \\
(\mathrm{D}=6 \\
\mathrm{mm})\end{array}$ & ICG & $1: 0$ & CW & $\begin{array}{l}\text { Laser } \\
\text { diode } \\
\text { (seq. } \\
\text { point) }\end{array}$ & $\begin{array}{c}\text { CCD camera } \\
\text { (area) }\end{array}$ & $3 \mathrm{D}$ & FEM & $\begin{array}{c}\text { Newton's } \\
\text { iterative } \\
\text { method }\end{array}$ \\
\hline
\end{tabular}




\section{APPENDIX C}

Summary of the experiment parameters for various experimental cases including the average measured source intensities, the gain setting on the image intensifier, the actual target location in $\mathrm{cm}$, the actual target volume, the measured optical property values of the phantom at the excitation and emission wavelengths, the target to background ratio (T:B), the recovered target location in $\mathrm{cm}$, the recovered $\mu_{\text {axf }}$ values in $\mathrm{cm}^{-1}$, the recovered target volume, the number of iterations executed before convergence, and the breakpoint (cutoff value selected on the first breakpoint of a histogram plot of the recovered $\mu_{\text {axf }}$ values). Results from experimental cases 26 and 27 (highlighted in yellow) are presented in Section 10.3 .

\begin{tabular}{|c|c|c|c|c|c|c|c|c|c|c|c|c|c|c|}
\hline Expt. & $\begin{array}{l}\text { Are. Source lat. } \\
\text { [\$1. \$2. \$3. \$4. } \\
\text { \$5. \$6] (EV) }\end{array}$ & Gais & $\begin{array}{c}\text { Act. Targ- } \\
\text { Location } \\
\text { [x.y.z] }\end{array}$ & $\begin{array}{l}\text { Acteal } \\
\text { Target } \\
\text { Yolene }\end{array}$ & $\mu_{m x}$ & $\mu_{n}=$ & $\mu_{2 x}^{\prime}$ & $\mu_{2-}$ & T:B & $\begin{array}{l}\text { Recorered } \\
\text { Target } \\
\text { Location } \\
\text { [x.y.z] }\end{array}$ & $\begin{array}{c}\text { Recore } \\
\text { red } \\
\mu_{\text {ed }}\end{array}$ & $\begin{array}{c}\text { Rec. } \\
\text { Targ. } \\
\text { Yol. } \\
\text { (CC) }\end{array}$ & $\underset{\text { Iter. }}{=}$ & $\begin{array}{l}\text { Break- } \\
\text { point }\end{array}$ \\
\hline $\mathbf{1}$ & $\begin{array}{c}0.614,0.580,0.068 \\
1.859,0.044,1.408\end{array}$ & 4.7 & $(4.0,2.5,1.0)$ & $0.45 \mathrm{cc}$ & 0.11 & 0.08 & 6.16 & 5.73 & perfect & $(3.96,2.71,0.73)$ & 0.0414 & 0.12 & & 0.032 \\
\hline 2 & $\begin{array}{c}0.614,0.580,0.068 \\
1.859,0.044,1.408\end{array}$ & 5.15 & {$[4.0,2.5,1.5]$} & $0.45 \mathrm{cc}$ & 0.11 & 0.08 & 6.16 & 5.73 & perfect & {$[3.91,3.07,0.70)$} & 0.1514 & 0.6 & & 0.223 \\
\hline 3 & $\begin{array}{c}0.614,0.580,0.068 \\
1.859,0.044,1.408\end{array}$ & 5.35 & $(4.0,2.5,2.0)$ & $0.45 c c$ & 0.11 & 0.08 & 6.16 & 5.73 & perfect & $\cdots$ & & $\cdots$ & & 0.003 \\
\hline 4 & $\begin{array}{c}0.057,0.535,1.533 \\
1.005,0.054,1.471\end{array}$ & 4.9 & {$[1.5,2.5,1.0)$} & $0.45 c c$ & 0.08 & 0.12 & 8.00 & 7.00 & perfect & $(2.11,2.92,0.57)$ & 0.0624 & 0.2701 & 6 & 0.073 \\
\hline 5 & $\begin{array}{c}0.057,0.535,1.533 \\
1.005,0.054,1.471 \\
\end{array}$ & 4.75 & {$[9.0,2.5,1.5]$} & $0.45 \mathrm{cc}$ & 0.08 & 0.12 & 8.00 & 7.00 & perfect & {$[7.64,3.24,0.58)$} & 0.163 & 0.4482 & 40 & 0.064 \\
\hline 6 & $\begin{array}{c}0.057,0.535,1.533 \\
1.005,0.054,1.471\end{array}$ & 5.1 & $(1.5,2.5,1.5)$ & $0.45 \mathrm{cc}$ & 0.08 & 0.12 & 8.00 & 7.00 & Perfect & $(2.46,3.33,0.20)$ & 0.0033 & 0.0417 & 4 & 0.04 \\
\hline $\mathbf{7}$ & $\begin{array}{c}0.057,0.535,1.533 \\
1.005,0.054,1.471\end{array}$ & 5.15 & {$[8.5,2.5,1.5]$} & $0.45 c c$ & 0.08 & 0.12 & 8.00 & 7.00 & perfect & {$[8.59,3.46,0.68)$} & 0.0312 & 0.1789 & $50(\max )$ & 0.044 \\
\hline 8 & $\begin{array}{c}0.057,0.535,1.533 \\
1.005,0.054,1.471\end{array}$ & 4.75 & No Target & $0.45 c c$ & 0.08 & 0.12 & 8.00 & 7.00 & no $\mathrm{ICG}$ & NIA & 0.0647 & NiA & $50(\max )$ & $\mathrm{NMA}$ \\
\hline 9 & $\begin{array}{c}0.057,0.535,1.533 \\
1.005,0.054,1.471\end{array}$ & 4.9 & No Torget & $0.45 c c$ & 0.08 & 0.12 & 8.00 & 7.00 & no $\operatorname{lCg}$ & NIA & 0.0981 & NIA & $50(\max )$ & NIA \\
\hline 10 & $\begin{array}{c}0.057,0.535,1.533 \\
1.005,0.054,1.471\end{array}$ & 5.1 & No Torget & $0.45 c c$ & 0.08 & 0.12 & 8.00 & 7.00 & no $\mathrm{ICG}$ & NIA & 0.0225 & NIA & 5 & $\mathrm{~N} / \mathrm{A}$ \\
\hline 11 & $\begin{array}{c}0.057,0.535,1.533 \\
1.005,0.054,1.471 \\
\end{array}$ & 5.15 & No Target & $0.45 \mathrm{cc}$ & 0.08 & 0.12 & 8.00 & 7.00 & no $1 \mathrm{CG}$ & $\mathrm{NIA}$ & 0.0176 & $\mathrm{~N} / \mathrm{A}$ & 5 & NIA \\
\hline 12 & $\begin{array}{c}0.057,0.535,1.533 \\
1.005,0.054,1.471\end{array}$ & 4.9 & {$[1.5,2.5,1.0]$} & $0.45 \mathrm{cc}$ & 0.08 & 0.12 & 8.00 & 7.00 & perfect & $(1.50,3.29,0.67)$ & 0.0549 & 0.2523 & 10 & 0.0675 \\
\hline 13 & $\begin{array}{c}0.057,0.535,1.533 \\
1.005,0.054,1.471\end{array}$ & 5.1 & $(1.5,2.5,1.5)$ & $0.45 \mathrm{cc}$ & 0.08 & 0.12 & 8.00 & 7.00 & perfect & $(2.50,3.22,0.23)$ & 0.0069 & 0.3329 & 3 & 0.0063 \\
\hline 14 & $\begin{array}{c}0.057,0.535,1.533 \\
1.005,0.054,1.471\end{array}$ & 5.2 & {$[2.0,2.5,1.5)$} & $0.45 c c$ & 0.08 & 0.12 & 8.00 & 7.00 & perfect & {$[1.52,3.37,0.85)$} & 0.0923 & 0.4955 & $50(\mathrm{max})$ & 0.045 \\
\hline 15 & $\begin{array}{c}0.057,0.535,1.533 \\
1.005,0.054,1.471\end{array}$ & 5.25 & $(2.0,2.5,2.0)$ & $0.45 c c$ & 0.08 & 0.12 & 8.00 & 7.00 & perfect & $(0.41,3.59,1.40)$ & 0.0707 & 0.2836 & $50(\max )$ & 0.1 \\
\hline 16 & $\begin{array}{c}0.057,0.535,1.533 \\
1.005,0.054,1.471\end{array}$ & 4.9 & {$[1.5,2.5,1.0)$} & $0.45 \mathrm{cc}$ & 0.08 & 0.12 & 8.00 & 7.00 & perfect & $(1.21,2.51,1.63)$ & 0.2965 & 0.5631 & 6 & 0.1335 \\
\hline 17 & $\begin{array}{c}0.057,0.535,1.533 \\
1.005,0.054,1.471\end{array}$ & 5.1 & {$[1.5,2.5,1.5)$} & $0.45 c c$ & 0.08 & 0.12 & 8.00 & 7.00 & perfect & $(2.10,2.71,1.69\}$ & 0.1021 & 2.8258 & 3 & 0.0064 \\
\hline 18 & $\begin{array}{c}0.057,0.535,1.533 \\
1.005,0.054,1.471\end{array}$ & 5.2 & $(2.0,2.5,1.5)$ & $0.45 c c$ & 0.08 & 0.12 & 8.00 & 7.00 & perfect & $(1.57,1.96,1.73)$ & 0.1193 & 0.1927 & $50(\max )$ & 0.248 \\
\hline 19 & $\begin{array}{c}0.057,0.535,1.533 \\
1.005,0.054,1.471 \\
\end{array}$ & 5.25 & $(2.0,2.5,2.0)$ & $0.45 \mathrm{cc}$ & 0.08 & 0.12 & 8.00 & 7.00 & Perfect & {$[1.07,3.10,2.07)$} & 0.1748 & 0.5666 & $50(\mathrm{mox})$ & 0.114 \\
\hline 20 & $\begin{array}{c}0.057,0.535,1.533 \\
1.005,0.054,1.471\end{array}$ & 5.1 & $(1.5,2.5,1.5)$ & $0.45 c c$ & 0.08 & 0.12 & 8.00 & 7.00 & perfect & $(2.46,3.33,0.20)$ & 0.0033 & 0.0417 & 4 & 0.04 \\
\hline 21 & $\begin{array}{c}0.057,0.535,1.533 \\
1.005,0.054,1.471\end{array}$ & 5.15 & $(8.5,2.5,1.5)$ & $0.45 \mathrm{cc}$ & 0.08 & 0.12 & 8.00 & 7.00 & perfect & $(8.59,3.46,0.68)$ & 0.0312 & 0.1789 & $50(\operatorname{mox})$ & 0.045 \\
\hline 22 & $\begin{array}{c}0.057,0.535,1.533 \\
1.005,0.054,1.471\end{array}$ & 5.2 & $(2.0,2.5,1.5)$ & $0.45 \mathrm{cc}$ & 0.08 & 0.12 & 8.00 & 7.00 & perfect & $(1.89,3.18,0.65)$ & 0.0202 & 0.4525 & $50(m a x)$ & 0.0183 \\
\hline 23 & $\begin{array}{c}0.057,0.535,1.533 \\
1.005,0.054,1.471 \\
\end{array}$ & 5.3 & {$[8.5,2.5,2.0)$} & $0.45 \mathrm{cc}$ & 0.08 & 0.12 & 8.00 & 7.00 & Perfect & {$[8.24,2.30,0.50)$} & 0.0063 & 0.1507 & $50(\operatorname{mox})$ & 0.0184 \\
\hline 24 & $\begin{array}{c}0.057,0.535,1.533 \\
1.005,0.054,1.471\end{array}$ & 5.25 & $(2.0,2.5,2.0)$ & $0.45 c c$ & 0.08 & 0.12 & 8.00 & 7.00 & perfect & {$[1.54,1.64,0.66)$} & 0.0011 & 0.1088 & $50(\max )$ & 0.0082 \\
\hline 25 & $\begin{array}{c}0.057,0.535,1.533 \\
1.005,0.054,1.471\end{array}$ & 5.25 & $(8.0,2.5,2.0)$ & $0.45 c c$ & 0.08 & 0.12 & 8.00 & 7.00 & perfect & {$[7.53,3.44,0.41)$} & 0.025 & 0.1765 & $50(m>x)$ & 0.056 \\
\hline 26 & $\begin{array}{l}0.614,0.580,0.068 \\
1.859,0.044,1.408\end{array}$ & 5.35 & $(4.0,2.5,2.0)$ & $0.45 c c$ & 0.11 & 0.08 & 6.16 & 5.73 & perfect & {$[4.58,2.33,0.20)$} & 0.003 & 0.0141 & 2 & 0.003 \\
\hline 27 & $\begin{array}{c}0.614,0.580,0.068 \\
1.859,0.044,1.408\end{array}$ & 5.4 & $(4.0,2.5,2.5)$ & $0.45 \mathrm{cc}$ & 0.11 & 0.08 & 6.16 & 5.73 & perfect & {$[3.71,3.21,0.70)$} & 0.003 & 0.1077 & 2 & 0.003 \\
\hline 28 & $\begin{array}{c}0.614,0.580,0.068 \\
1.859,0.044,1.408\end{array}$ & 5.4 & $(4.0,2.5,3.0)$ & $0.45 \mathrm{cc}$ & 0.11 & 0.08 & 6.16 & 5.73 & perfect & {$[4.14,4.18,3.95]$} & 1.1094 & 369.8 & 2 & 0.003 \\
\hline 29 & $\begin{array}{c}0.057,0.535,1.533 \\
1.005,0.054,1.471\end{array}$ & 5.35 & {$[6.0,2.5,2.0)$} & $0.45 \mathrm{cc}$ & 0.08 & 0.12 & 8.00 & 7.00 & perfect & {$[6.44,3.99,0.59)$} & 0.000 & 0.0913 & 2 & 0.003 \\
\hline 30 & $\begin{array}{c}0.057,0.535,1.533 \\
1.005,0.054,1.471\end{array}$ & 5.4 & $(6.0,2.5,2.5)$ & $0.45 \mathrm{cc}$ & 0.08 & 0.12 & 8.00 & 7.00 & Perfect & $\cdots$ & $\cdots$ & $\cdots$ & none & $\cdots$ \\
\hline 31 & $\begin{array}{c}0.057,0.535,1.533 \\
1.005,0.054,1.471\end{array}$ & 5.45 & $(6.0,2.5,3.0)$ & $0.45 \mathrm{cc}$ & 0.08 & 0.12 & 8.00 & 7.00 & Perfect & $\cdots$ & $\cdots$ & $\cdots$ & $50(m a x)$ & $\cdots$ \\
\hline
\end{tabular}




\section{SARAH J. ERICKSON}

2005-2011

2002-2005
Graduate Researcher, Department of Biomedical Engineering, Florida International University, Miami, FL

Bachelor of Science, Department of Physics, University of South Florida,Tampa, FL

\section{AWARDS}

1. Post-Doctoral Fellowship, American Cancer Society and Canary Foundation, 2011-2013

2. Pre-Doctoral Fellowship, Department of Defense Breast Cancer Research Program, 20082011

3. Research Excellence Travel Award, SPIE Photonics West, San Francisco, CA, 2011

4. Session Best Paper Award, 14 ${ }^{\text {th }}$ World Multi-Conference on Systems, Cybernetics and Informatics, 2010

5. $1^{\text {st }}$ Place Engineering Paper Competition Award, Scholarly Forum, Florida International University, 2010

6. Lydia I. Pickup Scholarship, Society of Women Engineers, 2009

7. $1^{\text {st }}$ Place Doctoral Student Paper Award, SBEC Paper Competition, $25^{\text {th }}$ Southern Biomedical Engineering Conference, 2009

8. $3^{\text {rd }}$ Place Best Student Poster Award NIH Inter-Institute Workshop on Optical Diagnostic and Biophotonic Methods from Bench to Bedside, Bethesda, MD, 2009

9. $3^{\text {rd }}$ Place Engineering Paper Competition Award, 2009 Paper Competition, Scholarly Forum, Florida International University

10. Presidential Fellowship, Florida International University Graduate School, 2005-2008

11. Derek Jacobs Memorial Scholarship, College of Engineering and Computing, Florida International University, 2008

12. Travel Award, Graduate Student Association, Florida International University, 2008

13. Aboly Foundation Scholarship, Physics Department, University of South Florida, 2005

14. Aboly Foundation Scholarship, Physics Department, University of South Florida, 2004

\section{PUBLICATIONS}

1. S.J. Erickson, S.L. Martinez, J. Gonzalez, L. Caldera, and A. Godavarty. "Improved detection limits using a hand-held optical imager with coregistration capabilities," Biomedical Optics Express 1, 126-134 (2010).

2. S.J. Erickson, J. Ge, A. Sanchez, and A. Godavarty. "Two-dimensional fast surface imaging using a hand-held optical device: in-vitro and in-vivo fluorescence studies," Translational Oncology 3(1): 16-22 (2010).

3. J. Ge , S.J. Erickson, and A. Godavarty, "Multi-projection fluorescence optical tomography using a handheld-probe-based optical imager: phantom studies," Applied Optics 49, 4343-4354 (2010)

4. S. Regalado, S. J. Erickson, B. Zhu, J. Ge, and A. Godavarty. "Automated coregistered imaging using a hand-held probe-based optical imager," Review of Scientific Instruments 81: $023702(2010)$.

5. J. Ge, S.J. Erickson, and A. Godavarty. "Fluorescence tomographic imaging using a handheld probe based optical imager: extensive phantom studies," Applied Optics 48(33), 64086416 (2009).

6. S.J. Erickson and A. Godavarty. "Hand-Held Based Near-Infrared Optical Imaging Systems: A Review" Medical Engineering and Physics 31, 495-509 (2009). 
7. J. Martin, S. Erickson, G.S. Nolas, P. Alboni, T.M. Tritt and J. Yang. "Structural and transport properties of $\mathrm{Ba}_{8} \mathrm{Ga}_{16} \mathrm{Si}_{\mathrm{x}} \mathrm{Ge}_{30-\mathrm{x}}$ clathrates" Journal of Applied Physics, 99, 044903 (2006).

8. S.J. Erickson, S. Martinez, J. Gozalez, M. Roman, A. Nunez, and A. Godavarty. "3D tomographic breast imaging using a hand-held optical imager," SPIE Photonics West, San Francisco, CA, Jan. 22-27, 2011.

9. J. Gonzales, J. DeCerce, S. Martinez, , S.J. Erickson, and A. Godavarty, "Simultaneous bilateral breast imaging using a novel handheld optical device," SPIE Photonics West BiOS, San Francisco, CA, January 22-27, 2011.

10. S.J. Erickson, S. Martinez, J. Gonzalez, L. Caldera, and A. Godavarty. "Non-invasive Diagnostic Breast Imaging using a Hand-held Optical Imager," Proceedings of the $14^{\text {th }}$ World Multi-Conference on Systems, Cybernetics and Informatics, 2010.

11. S. J. Erickson, S. Martinez, L. Caldera, and A. Godavarty, "Improved Detection Limits Using a Hand-Held Optical Imager with Coregistration Capabilities," in Biomedical Optics, OSA Technical Digest (Optical Society of America, 2010), paper BTuD43.

12. S. Martinez, J. DeCerce, J. Gonzalez, S. J. Erickson, and A. Godavarty, "Assessment of Tracking Devices towards Accurate Coregistration in a Hand-Held Optical Imager," in Biomedical Optics, OSA Technical Digest (Optical Society of America, 2010), paper BTuD58.

13. S.J. Erickson, S. Martinez, J. DeCerce, A. Romero, L. Caldera, A. Godavarty. "Fast coregistered imaging in vivo using a hand-held optical imager," Advanced Biomedical and Clinical Diagnostic Systems VIII. Edited by Vo-Dinh, Tuan; Grundfest, Warren S.; Mahadevan-Jansen, Anita. Proceedings of the SPIE, Volume 7555, pp. 75550P-75550P-6 (2010).

14. S.J. Erickson, J. Ge, and A. Godavarty. "Clinical Translation of a Novel Hand-Held Based Optical Imager: In Vitro and In Vivo Studies," IFMBE Proceedings 25th Southern Biomedical Engineering Conference 2009, 15 -- 17 May 2009, Miami, Florida, USA; 24: 34; A.J. McGoron, C.Z. Li, and W.C. Lin, eds. ISBN: 978-3-642-01696-7; 2009.

15. J. Ge, S.J. Erickson, and A. Godavarty. "Fluorescence Tomographic Imaging Using a Hand-Held Optical Imager: Extensive Phantom Studies," IFMBE Proc. 25th Southern Biomedical Engineering Conference 2009, 15 -- 17 May 2009, Miami, Florida, USA; 24: 12; A.J. McGoron, C.Z. Li, and W.C. Lin, eds. ISBN: 978-3-642-01696-7; 2009.

16. S.J. Erickson, S. Regalado, J. Ge, B. Zhu, A. Godavarty. "Self-coregistration in a novel hand-held based optical imager towards early-stage breast cancer detection," Proceedings of SPIE, Advanced Biomedical and Clinical Diagnostic Systems VII, SPIE Photonics West, San Jose, CA; 7169: 716914, 2009.

17. J. Ge, S.J. Erickson, A. Godavarty, "Multi-projection based fluorescence optical tomography using a hand-held probe based optical imager," Proceedings of SPIE Photonics West, Advanced Biomedical and Clinical Diagnostic Systems V, SPIE Photonics West, San Jose, CA, Jan. 24-29, 2009.

18. A. McGoron, J. Wang, S. Erickson, M. Gorywala, "Quantitative comparison of two gating schemes in lung PET: simulation with computer phantom," Biomedical Engineering Recent Developments; Proc. of the $24^{\text {th }}$ Southern Biomedical Engineering Conference, $\mathrm{H}$. Nazeran, M. Goldman, R. Schoephoerster, eds. ISBN: 978-1-93063607-1:2008.

19. J. Martin, S. Erickson, G.S. Nolas, P. Alboni and T.M. Tritt. "Thermoelectric properties of Ba-filled Si-Ge alloy type I semiconducting clathrates" Proceedings of the 24th International Conference on Thermoelectrics, Clemson, SC, 19-23 June 2005.

20. G.S. Nolas, M. Beekman, J. Martin, H.F. Rubin, S. Erickson, G.A. Lamberton, Jr and T.M. Tritt. "Research on "Open-Structured" Materials for Thermoelectric Power Generation" $23^{\text {rd }}$ International Conference on Thermoelectrics, Adelaide, Australia 25-29 July 2004.

Conference Presentations (not listed): 12 Oral, 5 Poster 